







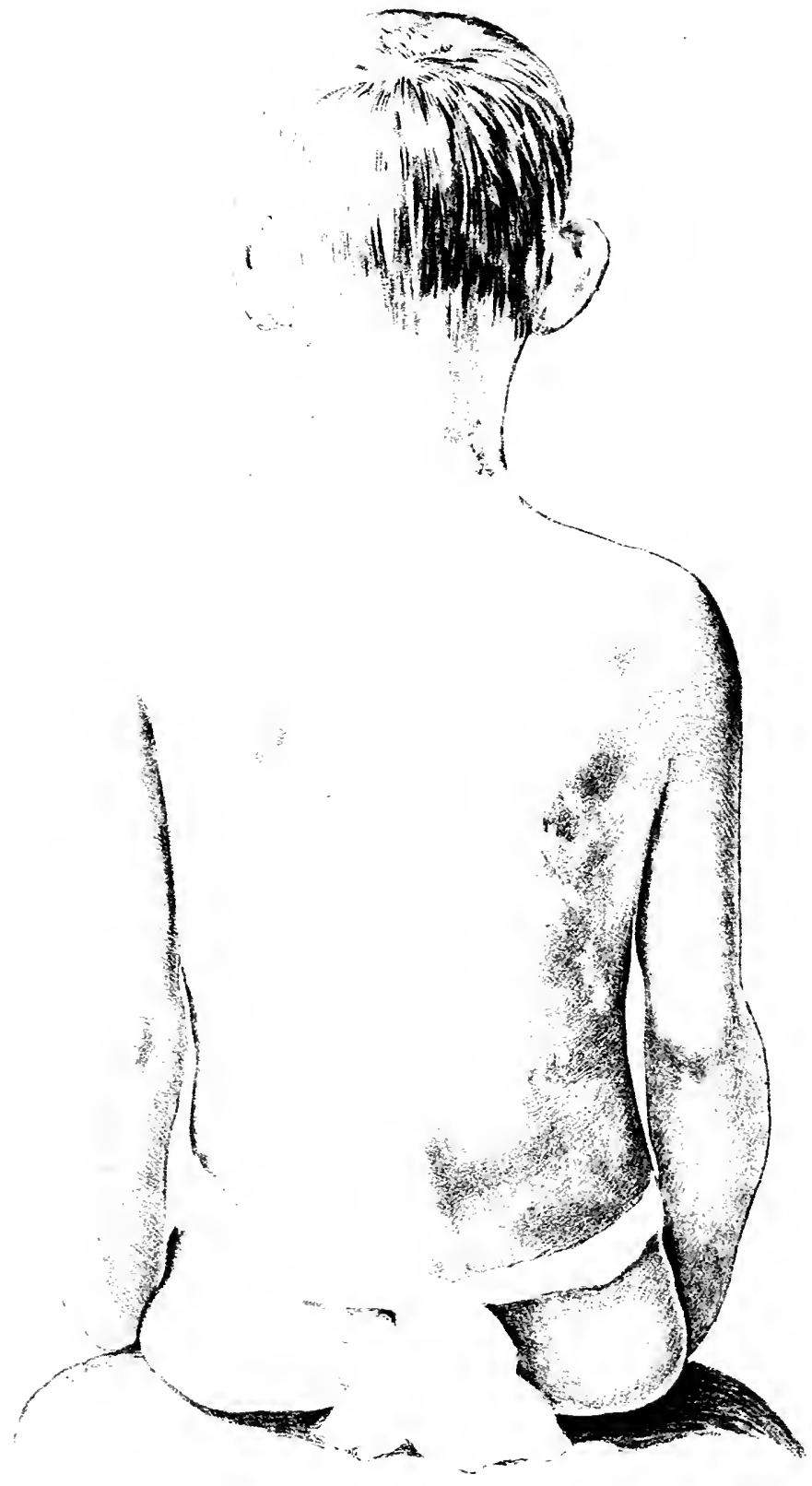

PIGMENTARY SYPHILIDE AND ALDPECIA (Authar's casa fram King's Cuunty Haspital), Patiant was a famala, aged saven years, with acquired Syphilis, and presented a diffuse, wide-spraed dark pigmentation over body and neck, with ereas of white healthy skin scattered here and there through it. The Alapecia accurred in the fanm of nathige of haltagon 


\section{GENITO-URINARY DISEASES}

\section{AND SYPHILIS}

$\mathrm{BY}$

\section{HENRY H. MORTON, M.D.}

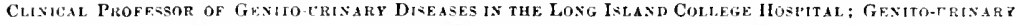

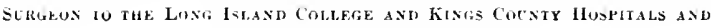

THE POLHEMTS MEMORIAL CEINIC.

\section{Illustrated with Galf-tones and Full=page Color:plates}

\section{PhiLADELPHIA}

F. A. DAVIS COMPANY, PLBLISHERS 1903 
Entered According to Act of Congress, in the Year 1902 BY $11 \mathrm{EXRY}$ 11. MORTUN.

in the office of the Labrarian of Congress at Washington. D. C. ALL RIiUIS RESERTED.

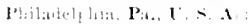

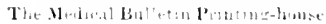
1.th-lictietristient. 
TO THE MEMORY

OF

MY OLD TEACHER AND FRIEND

\section{Dr. Alexander J. C. Skene}

IN RECOGNITION OF HIS BRILLIANT ABILITIES AS A SURGEON

AND IN AFFECTIONATE REMEMBRANCE OF HIS PERSONAL QUALITIES AS A MAN

THIS WORK IS DEDICATED 



\section{PREFACE.}

In the past ten years no branch of surgery or medicine has made greater progress than the department of genito-urinary surgery. In that short period of time, the treatment of acute and chronic gonorrhœa has been removed from mere empiricism and placed upon a scientific and rational basis. This has been accomplished through investigations, whose results have given us a definite knowledge of the micro-organisms concerned, and the pathological changes in the urethral tissues which their presence excites.

The whole subject of chronic seminal vesiculitis, with its relation to sexual neurasthenia, and the ever-present danger of lurking infection, has been clearly demonstrated. It is less than ten years since the cystoscope came to be of practical use, and ont of its derelopment grew the various instruments for collecting the urine from each kidney separately, in this way stimulating a greater interest in the subject of renal surgery.

While the operations for stone in the bladder are as old as civilization itself, the improvements in the technique of lithotomy, and a clearer comprehension of the indications for each form of operation. are matters of very recent growth. Ten years ago the cases of hypertrophied prostate in old men were without remedy, after the failure of the catheter to alleviate the urgent symptoms, but to-day the operations of prostatectomy, castration, and Bottini's operation have opened a way of relieving the suffering and prolonging life.

The above-mentioned adrances are only a few of the steps in the progress of this important branch of surgery. 
In this little rolume the author has endeavored to present, in a concise form, the present status of genito-urinary diseases and syphtis... At the same time he has endeavored to keep in mind the needs Lactitioner, whose opportunities for seeing such cases may be infrequent, and to consider the questions of diagnosis, prognosis, and treatment in such a way that the book may be of practical use in these matters.

40 SCIIERMERHORN STREET, BROUKLYN-NEW YORK. 


\section{CONTENTS.}

DISEASES OF THE PENIS.

Chapter I.-Phimosis. Balano-posthitis. Herpes Progenitalis. Papillo-

mata. Cancer of the Penis.............. 1

DISEASES OF THE URETHRA AND ITS ADNEXA.

Chapter 11.-Anatomy of the Urethra................... 16

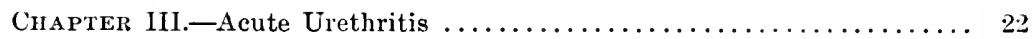

Chapter IV.-Posterior Urethritis $\ldots \ldots \ldots \ldots \ldots \ldots \ldots \ldots \ldots \ldots \ldots \ldots \ldots$

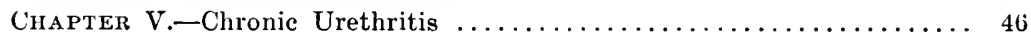

COMPLICATIONS OF ACUTE GONORRHEA.

Cnapter VI.-Balanitis. Phimosis.' Paraphimosis. Folliculitis. Cowperitis. Inguinal Adenitis. Chordee. Epididymitis. Gonorrhœal Rheumatism ............... 75

INFLAMMATIONS OF THE PROSTATE.

Chapter VII.-Acute Prostatitis. Chronic Prostatitis............. 84

DISEASES OF THE SEMINAL VESICLES.

Chapter VIII.-Acute Seminal Vesiculitis. Chronic Seminal Vesiculitis. Tuberculous Vesiculitis ................ 91

STRICTURE OF THE URETHRA.

Chapter IX.-Spasmodic Stricture. Organic Stricture. Treatment of Stricture by Surgical Operation. Extravasation of

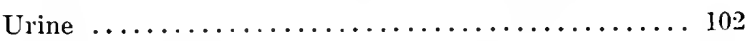

Chapter X.-Urinary Fever. Care of Urethral Instruments.......... 130

DISEASES OF THE BLADDER.

Chapter XI.-Cystitis. Bacteriuria. Tumors of the Bladder......... 134

Chapter XII.-Vesical Calculus . . ................... 159 
DISEASES OF THE PROSTATE.

Cinapter XIII.-Senile Ilypertrophy of the Prostate............. 181

CHApter AIV.-Operative Treatment of Hypertrophied Prostate...... 202

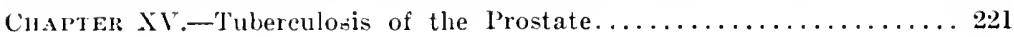

DISEASES OF THE KIDNEYS.

Chapter XVI.-Movable Kidncy. Renal Caleulus. Pyelitis. Hydronephrosis $\ldots \ldots \ldots \ldots \ldots \ldots \ldots \ldots \ldots \ldots \ldots \ldots \ldots \ldots \ldots$

DISEASES OF THE TESTICLES.

Chapter XVII.-Ectopy of the Testicle. Malignant Disease. Tubercu-

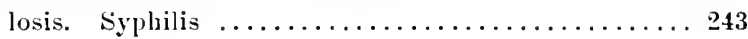

HYDROCELE, HAMATOCELE, AND VARICOCELE.

Cinaptel: XVili.-Hydrocele. Hæmatocele. Varicocele.

\section{CHANCROID AND ITS COMIPLICATIONS.}

Chapter XIX.-Chaneroid. Complications 271

\section{SIPHILIS AND ITS LESIONS.}

Chapter XX.-Chancre. Abortion of Syphilis After Infection......... 284

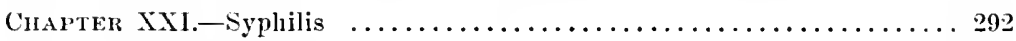

C'IAPTER XXII.-Treatment of Syphilis..................... 327

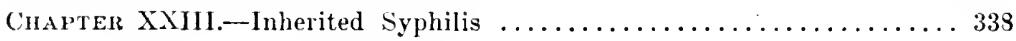

\section{IMPOTENCE AND STERILITY.}

Chapter XXIV.-Impotence. Sterility

List of Genito-urinary Instruments Required for Office Use......... 363

Index 


\section{LIST OF ILLUSTRA'TIONS.}

Colored Plate. Pigmentary Syphilide and Alopecia. (Author's Case.)

Frontispiece

1. Papillomata-Venereal Warts. (Author's (ase.)........... 9

2. Diagram of Blader and Urethra....................... 1 i

3. Diagram of bladder and Urethra.................... 19

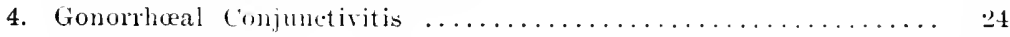

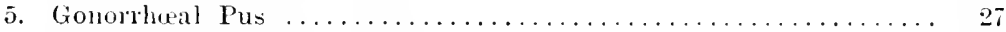

6. Gonococei Growing in Gluster............................

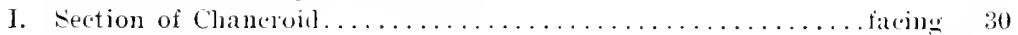

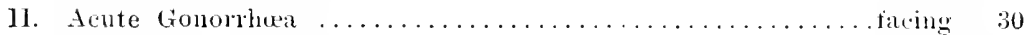

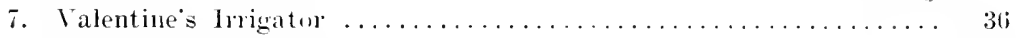

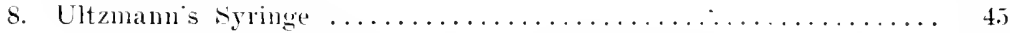

9. Diagram of a Cross-section of the Urethra, lieprencnting the Hinto-

logical Changes in Chronic Urethritis................ 47

10. Diagram of a Section of the Urethra, Repesenting the Histological Changes in the Formation of a Cirmular Patch in Chronic Ure-

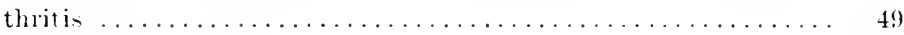

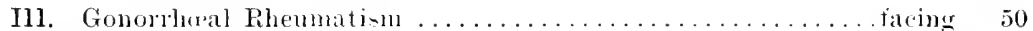

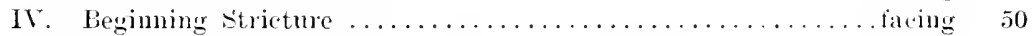

11. Shred from a case of Gonorrhera of lone standine.......... 52

12. Otis Urethrometer ............................ 5

13. Diagram showing method of detecting Deep Infiltration in Chronic Urethritis, with Bougie i lBoule or Urethrometer.......... 53

14. Diagram showing impossibility of recognizing Superficial Infiltration, involving Nueons Nembrane alone, by mans of Bougie a Boule or Urethrometer .......................... 53

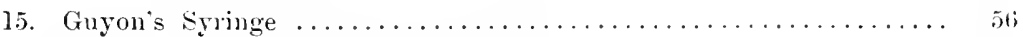

16. Steel Sound with Tan Buren Curve................... $5 !$

17. Psyehrophor, or Cold-Water Somul, of Wintemitz........... (i.)

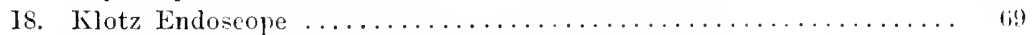

19. Urethroscopic picture of a Normal Uretha, showing a multitude of fine folds and small Central Figure................ i0

20. Urethroscopie picture of Soft Infiltration of the Nucous and Sub-

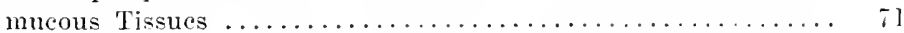

21. Urethroseopic picture of Hard Infiltration of the submuroms likenes.

22. Periurethral Abscess, begiming as a Folliculitis. (Author's (ane.). TT

23. Horand-Langlebert Suspensory bandage.............. so

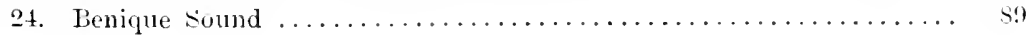


25. Diagram of the Seminal Vesicles. The liight Vesicle has been Dissected, and its Convolutions Drawn Out straight............

26. Microscopic examination of mattrial expressed from Seminal Vesieles and frontate, showing spermaturoar, Puscells, and boettscher's

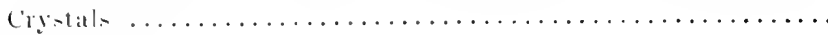

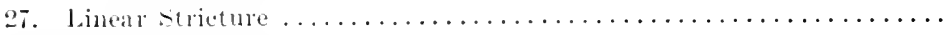

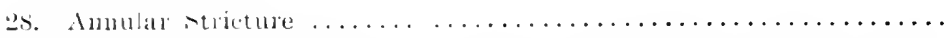

99. Changes behind a stricture. Dilatation of ponch inmediately behind stricture. Ilypertrophy and Contration of Bladder. Dilatation

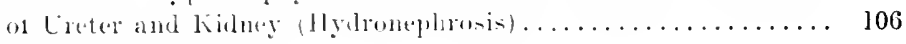

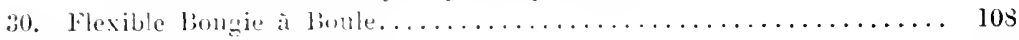

31. Jletal liungies à bunle.......................... 109

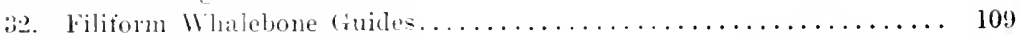

33. Strieture of the Bullu-memhranous Utedha and False l'assage.... 111

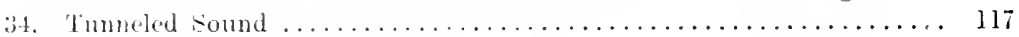

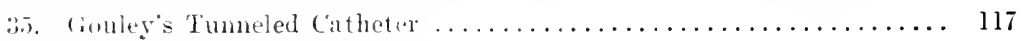

3ti. (1) Unethrotone, as Muditied by Rame................ 119

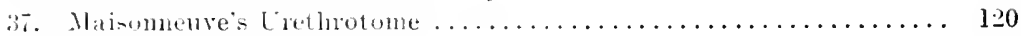

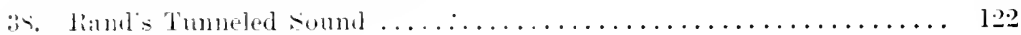

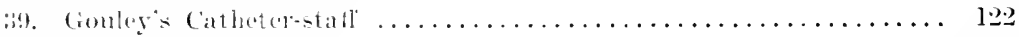

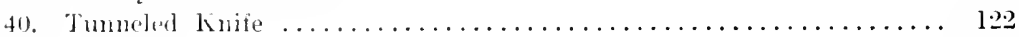

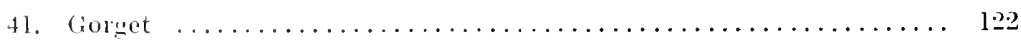

42. Lxternal Urethrotomy. Wheelhomse Operation, Expusing the Ure-

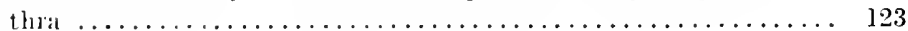

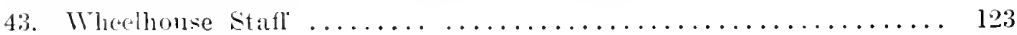

44. Small Tenaculum for Holding Alunt Inei-od Cretlua in External

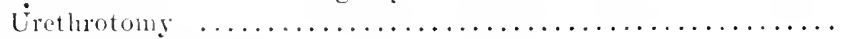

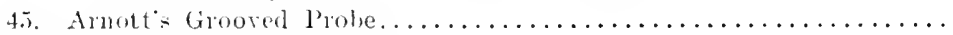

4ti. Gouley's Beaked Bintoury $\ldots \ldots \ldots \ldots \ldots \ldots \ldots \ldots \ldots \ldots \ldots \ldots$

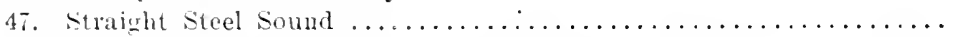

45. Fornation of a Sacculation in a lypertropliced Bladder from l'ro-

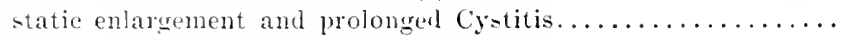

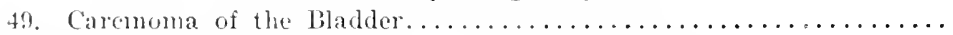

50. Oxalie or Mhlbery Caleulus, (Authors Specimen.)............

51. Vesical calculus. split in two Halves, slowing mode of formation, by a deposit of l'hosphates in Concentric Layers, around a Uric-

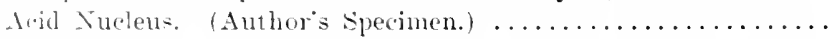

id. Multiple l'hosphatic (alculi, removed by Suprapulsic Cystotony from rane l'atient. (Author's specimen.) $\ldots \ldots \ldots \ldots \ldots \ldots \ldots \ldots$

53. Caleuli which formed in the Bladder as a Single Stone, which under"rot sununous liracture. (Author's Specimen.)........ 163

it. Thumpon's Searcher for Vesical Calculus............... 166

a.). Curchliner for stoue Lying in the Post-prostatic Pouch.......... 167

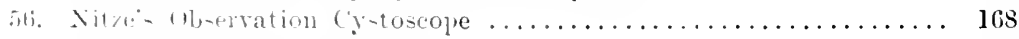

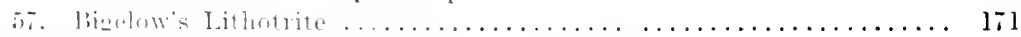

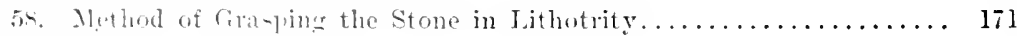

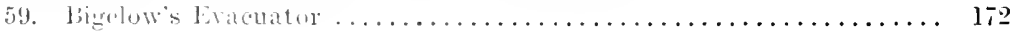


!o. Cimmata of the Tongue. The one in the middle has mudergone Congulation-necrosis of its centre. (Author's Case.) ....... 311

9). Ulerrating Gumma of the Ankle. (Author's Case.).......... 311

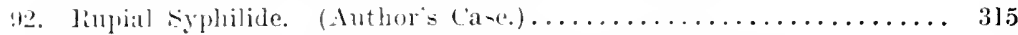

93. Pustular syphide (Malimant syphilis). (Author's Case.)....... 317

( alored Plate. Dry or Atrophie Tuberenlar Syphilide. (Anthor's Case.)

facing 334

94. Hutchinson's Treth. These Teeth have been recently eut, and the Central Notch is well outlined, but the thin and umprotected

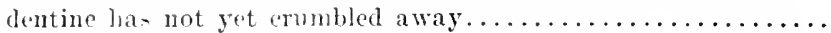

95 and 9t. Hutrhincon's Teeth, showing later Stages of the Process

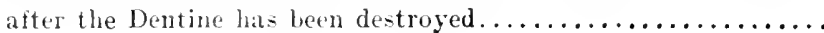




\section{DISEASES OF THE PENIS.}

\section{HAPTER I.}

PHIMOSIS.

BY the term phimosis is understood an abnormal narrowing of the opening of the prepuee, which prevents the retraction of the foreskin and causes the glans penis to be permanently covered. Phimosis may be congenital or may be acquired in adult life.

In nearly all male children at birth the foreskin is long and is adherent to the glans. In early life these adhesions are very weak and are easily ruptured by erections of the penis or manipulation, and in the course of the first few years the preputial orifice becomes enlarged and the prepuee can be stripped back over the glans. If the alhesions are not ruptured early, they become firmer as they grow older, and prevent the complete retraction of the foreskin.

The acquired form of phimosis occurs in adults, and often results from the cicatricial contraction of the margin of the prepnce following the healing of a chancroid in this location.

Temporary phimosis often results from the swelling and œedema of the prepuce which occurs in the course of an attack of gonorrhea or subpreputial chancroids.

As direct results of phimosis the following conditions are met with:-

(a) Balanitis and vencreal warts, resulting from the maceration of the epithelium in the balano-preputial sac, from retention of smegma and urine. On account of the difficulty of retracting the prepuce, proper cleanliness camnot be observed, and the tencter mucous membrane is especially liable to persistent and recurrent attacks of inflammation. Proliferation of the epithelial cells oceurs, and venereal warts grow luxuriantly.

(b) Preputial calculi, or concretions, form not infrequently. from a calcification of the smegma and the decomposition of the urinary salts. 
(c) Epithelioma of the penis is strongly predisposed to by the continued irritation of the foreskin and retained secretions under it.

(d) Arrested development of the penis usually occurs in consequence of the malnutrition from which the organ suffers.

(e) I'remature erections and serual excitement are generally noted in foung children affected with phimosis, and the continued irritation about the head of the penis often establishes a habit of masturbation.

(f) Interference with the act of coitus, often accompanied by premature ejaculation, is frequently complained of by adults affected with phimosis.

(g) Liability to infection with syphilis or chancroidal poison is invariably the case when the prepuce is long, even though it can be retracted, and Hutchinson points out that the circumcised Jew is less liable to contract syphilis than an uncircumcised person, because after circumcision the integument of the glans becomes horny, and not liable to abrasions.

The remote results of phimosis are equally important, and often present great difficulties in the exact determination of their origin.

(a) Retention or incontinence of urine, especially in children, but sometimes in adults, is often caused by a spasmodic contraction of the cut-ofr musele or an irritable bladder, induced by the irritation about the glans penis.

(b) IItmorrhoids, prolapsus ani, hernia. and dilatation of the ureters and lidney pelres often follow the prolonged and violent straining efforts which individuals affected with phimosis make, upon urinatirg, in order to overcome the resistance offered by a spasmodic stricture or a pin-point opening through the prepuce. The susceptib]e nervous sytem of children renders them particularly liable to the above-mentioned difticulties and also to the following affections:-

(c) Iffections of the nerrous system,-spastic palsies, simulated hip-joint disease. muscular inco-ordination, and convulsions,-which are often observed in young children as reflexes from a tight or adherent prepuce, and a disappearance of these symptoms often follows circumeision.

(d) IIerpes preputialis is another manifestation of the reflex action upon the nervous system and skin of the prepuce occasioned by the irritation of a long foreskin, even though it is possible to retract it over the glans. 


\section{TREATMENT.}

On account of the manifold advantages offered by an absence of the foreskin, it would be well if Christian nations were to arlopt the old Hebrew rite of circumcising all male children on the eighth day after birth. In this operation the foreskin is simply snipped off with one sweep of a knife, and the wound washed with a weak astringent antiseptic. The pain is slight. and an anæsthetic is not required, nor would it be safe to administer one in so young a child. Hæmorrhage is tritling, and there is but little risk of infection afterward.

Stripping back the foreskin and breaking up the adhesions is sometimes recommended, but is a very poor makeshift for cireumcision, and there is always the danger of the prejuce being caught back behind the glans, and becoming swollen and constricted, causing paraphimosis.

Circumcision is the operation of choice, and may be performed in two ways:-

(a) Circumcision with a Clamp.-In this operation the clamp is applied to the foreskin immediately below the glans penis, and all the prepuce which projects beyond the clamp is serered with a knife or scissors. This ineision simply cuts through the skin alone, and the mucous membrane lying next the glans is left intact and must next be trimmed off with the scissors. After this is done the cut edges of skin and mucons membrane are stitched together with interrupted sutures of catgut, and the operation is completed.

There are certain objections to this procedure. In applying the clamp, if too much traction is made on the foreskin, the slin of the proximal end retracts close up to the root of the penis after the incision is made, and leaves a gaping raw surface of mucous membrane to be covered. On the other hand, if too much foreskin is left, the glans remains covered by it and the object for which the operation was performed is not accomplisher. Eren when the incision is properly made the subsequent adjustment of skin and mucous membrane often induces a certain amount of tension and dragging upon the sutures, which interferes with the healing of the wound. For these reasons the author prefers the operation of

(b) Circumcision by Dorsal Incision and Trimming off the Flaps.-The surgeon stands upon the patient's left side and with a pair of straight scissors makes an incision on the dorsum of the prepuce, cutting through skin and mucous membrane at the same 
time. The incision is carried up to the point where the mucous membrane is reflected npon the glans penis. A grooved director may be introdnced under the foreskin if desired, to act as a guide for the suissors.

After the dorsal incision is made the two remaining flaps of skin and muleons membrane are trimmed off with eurved scissors. This incision is carried around the penis on both sides, following the line of insertion of the mucous membrane at a distance of oneeighth of an inch from the gians.

The frenum is divided last, and its artery, together with the dorsal artery, and sometimes one or two smaller ones, are picked up and tied, and the sutures are introduced.

It is desirable to place the first suture on the dorsum of the penis. and the sccond suture stitches the remains of the frænum to the point of skin underneath it. These anchor the skin and prevent it from shifting, as it lies on the mucous membrane. The other sutures are then introduced, and usually three on a side will be found enough. Fine catgut is the best material, as it is absorbed, while if silk sutures are used much trouble is experienced in remoring them.

The best form of dressing seems to be a piece of lint wet with an antiseptic solution and changed frequently, as any sort of permanent dressing is soon soaked with urine. The patient should remain quietly in bed or on a lounge, for a week, and may be then allowed to gro about as usual.

With regard to the anssthetic used, the operation may be done painlessly with cocaine, injected hypodermically under the skin of the penis along the line of incision, and in order to anesthetize the mucons membrane $1 \%$ drachm should be injected into the balanopreputial sae and held there for five minutes. The cedema of the collular tissue resulting from tying a rubber bandage around the root of the penis to prevent the too rapid absorption of the coeaine interferes with the best healing of the wound afterward, and on that account the author prefers to administer a general anæsthetic. $A$ better result will be ohtained if the patient is kept in bed for a few days following the operation than if allowed to be up and about his room. 


\section{BALANO-POSTHITIS.}

Balanitis consists in an inflammation of the mucous membrane covering the glans penis, and an inflammation affecting the mucous layer of the prepuce is termed posthitis. The inflammation of both surfaces usually exists simultaneously, and should be considered together.

Balano-posthitis cannot oceur in an individual who has been circumcised, but the presence of a long and phimotic foreskin allows the retention of the natural secretion of smegma and a few drops of urine, which decompose and irritate the already macerated mucous membrane, lowering its power of resistance to germ-infection.

Gouty and lithrmic conditions and diabetes also render the patient extremely liable to develop inflammation of the mueous membrane underneath a long foreskin.

As a direct exciting cause it is probably neeessary that microorganisms of some sort must be incenlated, and for this reason a balano-posthitis often develops from contact with irritating vaginal secretions in coitus or the aceidental introduction of pyogenic organisms from contact with the hands or elothing.

Chancre, gonorrhoa, and chancroid are apt to be complicated by balano-posthitis as a result of mixed infeetion, when they occur in an individual having a long foreskin.

\section{SYMPTOMS AND COURSE.}

A mild form of balano-posthitis is liable to occur at frequent intervals unless the man with a long foreskin attends with scrupulons care to drawing it back and washing it, and the preputial sac, at frequent intervals.

If this is not done, a sense of heat and itching is noticed at the end of the penis; the mucous membrane becomes hyperamic, infiltrated, and eroded; and a creamy-yellow, purulent discharge, with an extremely offensive odor, is secreted from the mucous membrane of the preputial sae.

In severe cases the excoriations are extensive and well marked and the inflammation and redema are extreme; so that the whole prepuce becomes swollen. In this condition gangrene of the foreskin, either in part or as a whole, not infrequently takes place. 


\section{DIAGNOSIS.}

The diagnosis of balano-posthitis presents but little difficulty, when the foresin can be retracted and the glans inspected; but when phimosis exists the diagnosis is often perplexing.

If a microscopie examination of the discharge fails to reveal gonnencei, but discloses numerons staphylococci, gonorrhcea may be exchuled.

Chancroid may be diagnosed by inoculating some of the preputial discharge upon the patient's thigh, and, if other chancroids are caused, it is probable that the original sore was a chancroid, although it is possible to cause sores resembling chancroids by the inoenlation of staphylococci.

Chancre can be excluded by the absence of an indurated mass muder the prepuce and the lack of the characteristic enlargement in the inguinal glands.

Epithelioma is often difficult to differentiate from the ehronic form of balano-posthitis which affects middle-aged men; but epithelioma does not respond to local treatment, while balanitis improves quickly. In cases of doubt it is always in order to excise a small portion of the prepuce and subject it to microscopic examination to determine the question.

\section{TREATMENT.}

The essential points in the treatment are to keep the parts clean and dry. These indications can be met in the following manner:-

In the cases when the prepuce can be retracted, the balanopreputial sac should be washed out with a mild antiseptic solution, either bichloride ( 1 in 10,000 to 1 in 4000 ) or Thierseh's fluid. The parts should then be dried and covered with a dusting-powder:-

R Pulv. amỹli,

Pulv. zinci oxidi,

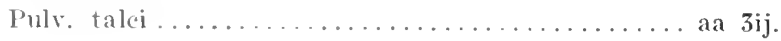

() $\mathrm{r}:-$

R Hydrarg. chlor mite. ................... gr. xxx.

Acidi borici............................

Acidi salicylici...................... gr. v.

In chronic cases, occurring in elderly men, subgallate of bismuth has a particularly good effect. 
After applying the dusting-powder the glans should be covered with a layer of cotton and the foreskin drawn forward into place. This dressing should be changed several times a day.

If the erosions are deep and extensive, their healing can be hastened by brushing them over with a 10-per-cent. nitrate-of-silver solution before applying the dusting-powder.

When phimosis exists and the foreskin cannot be retracted, a long, flat-billed syringe should be used for washing out the balanopreputial sac, every few hours.

A few syringefuls of warm water and soap may be thrown in and followed by injecting bichloride solution (1 in 10,000) or Thiersch's fluid.

The cedema and swelling of the parts may be mitigated by prolonged soaking in hot water.

If the balanitis occurs as a result of diabetes or a subpreputial chancroid, it may be necessary to relieve the tension by slitting up the prepuce on the dorsum in order to avert impending gangrene.

In all cases of chronic or relapsing balano-posthitis, in addition to local measures, attention should be directed to the diathetic conditions which prevent a permanent healing. If the individual is gouty or diabetic, a suitable regimen should be adopted, and the general health carefully looked after.

In obstinate cases of balano-posthitis, circumcision should be performed as soon as the acute symptoms have subsided. This is especially necessary in elderly men, who are at an age when epithelioma is liable to develop on the glans or under the prepuce from the prolonged irritation of the parts.

In diabetic patients the operation of circumcision should be avoided if possible and the danger of extensive gangrene following slight operations should be borne in mind. If any operative procedure is demanded, it is of the highest importance to get the patient's urine in good condition before operating. 


\section{HERPES PROGENITALIS.}

This affection is characterized by the formation of groups of small vesicles upon an erythematous base and located on the skin or mucous surface of the prepuce.

The thin resicles are easily ruptured, and leave small, round, shallow, punched-ont ulcers, which heal spontaneously in a few days.

Unlike herpes zoster, herpes progenitalis is generally unaccompanied with pain. In exceptional cases, however, pain is felt, which is neuralgic in character and precedes the appearance of the eruption.

A urethral discharge is sometimes observed, and endoscopic examination shows a collection of vesicles located within the urethra.

Recurrence is a marked characteristic of herpes progenitalis, and it is the rule for patients to have several attacks a year.

\section{ETIOLOGY.}

Herpes progenitalis may be regarded as a reflex manifestation of some irritation of the nerres supplying the genitals, and is usnally due to balano-posthitis, excessive coitus, or a long prepuce. Gouty and lithamic conditions are thought to predispose the patient to atticks of herpes.

\section{DIAGNOSIS.}

If the ease is scen early, the appearance of the resicles is unmistakable, and after they have ruptured the small, round, punchednut ulcers are quite characteristic.

The lymphatic glands in the groin are usually not affected, but in one caso in ten the inguinal glands are said to be enlarged in a chain, as in syluitis. (.T. IT. White.)

\section{TREATMENT.}

The herpetic losions raphly heal with eleanliness and the application of a simple absorbent dusting-powder. Recurrent herpes progenitalis, which ahmot always oceurs in connection with a long foreskin, can only be juerented by the operation of circumcision. 


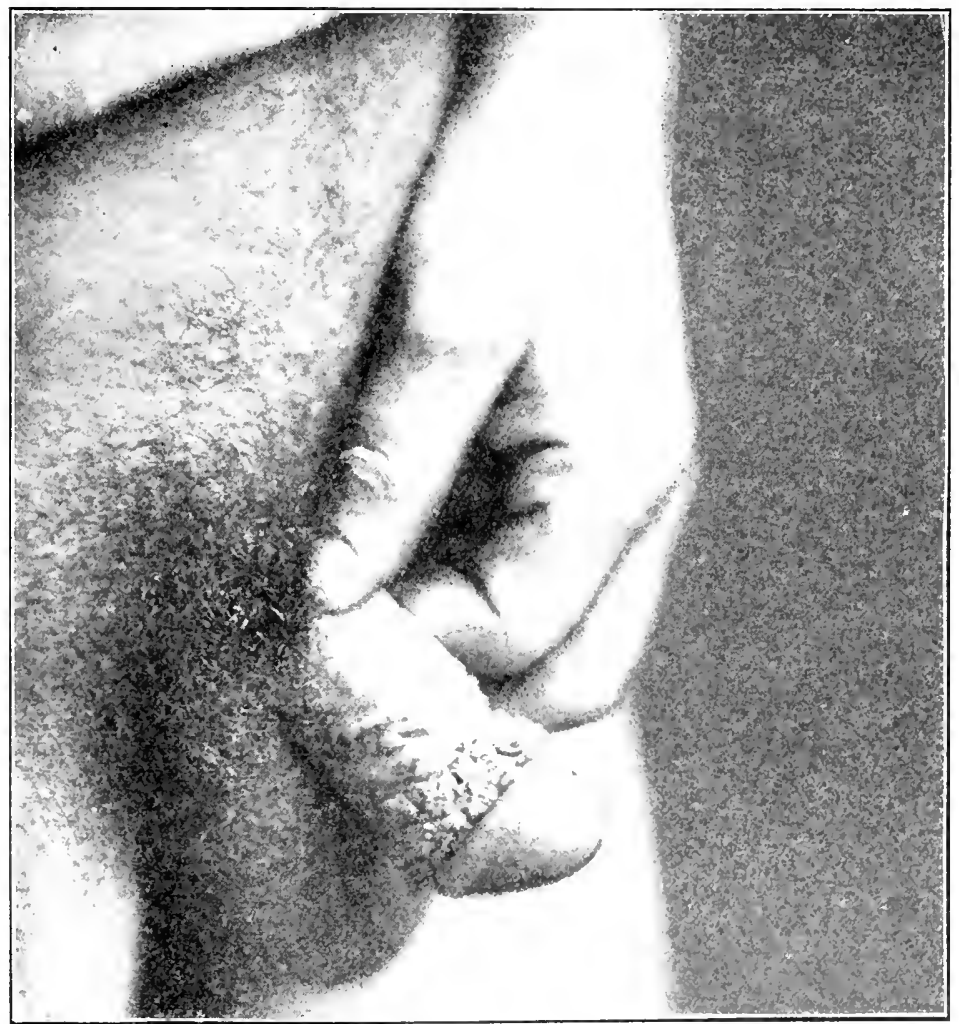

Fig. 1.-Papillomata-Tenereal Warts. (Author's Ciase, frum King- (ounty Hospitil.) 



\section{PAPILLOMATA.}

Papillomata occurring about the head of the penis are frequently termed renereal warts. They are sometimes spoken of as condylomata, which is manifestly ineorrect, as true condylomata are a manifestation of syphilis, and the papillomata have no connection with syphilis, but are of purely local origin.

Papillomata consist in warty growths, which are flat or often cauliflower-like excrescences, usually located in the coronary sulcus under a long prepuce. In structure they are a simple hypertrophy of the papillary layer, and are caused by the prolonged maceration and softening of the mucous surfaces under a long foreskin, occasioned by contact with irritating discharges from gonorrhœa, chancroids, or balano-posthitis.

\section{DIAGNOSIS.}

Papillomata may be mistaken for the condylomata of syphilis or epithelioma. In syphilitic condylomata, however, other signs of specific disease are always present; but epithelioma may be difficult to differentiate from simple papillomata, and every warty growth occurring about the glans penis in elderly men should be regarded with suspicion.

\section{TREATMENT.}

Small warts sometimes disappear if the parts are kept clean and covered with a dusting-powder; but their disappearance is a matter of uncertainty, and always very slow. Operation is the best treatment, and should always be advised.

As papillomata occur in consequence of a long foreskin, circumcision should be performed, and the warts which are not removed with the prepuce scraped off with a sharp curette. In order to prevent a recurrence, it is desirable to cauterize the bases with nitric or carbolic acid or the Paquelin cautery. 


\section{CANCER OF THE PENIS.}

Malignant discase of the penis occurs almost invariably as epithelial carcinoma, and begins with about equal frequency on the inner surface of the prepuce or upon the glans.

According to Jacobson, its mode of commencement is varied, but it appcars most frequently as: (a) A wart, or warty excrescence. Sometimes, however, it makes its appearance as: (b) A small nodule, or knot of induration. under the surface of the mucous membrane.

Again, epithelioma is observed occurring under the form of (c) a superficial excoriation, or raw patch, resembling the erosions found in balano-posthitis; or it may develop as $(d)$ an ulcer resulting from the transformation of a chancroid or the breaking down of an old cicatrix, or sometimes from a crack or tear on the margin of a tight foreskin.

In cases of extreme rarity epithelioma of the penis develops from the extension of the malignant process outward from the urethra or upward from the scrotum.

\section{ETIOLOGY.}

Under the head of predisposing causes age plays an important rôle, and epithelioma of the penis is very rarely found except between the fiftieth and seventieth years.

The next most important predisposing cause is phimosis. Demarquay found that out of fifty-nine cases of epithelioma of the penis, forty-two had long and phimotic foreskins, and many authors have called attention to the fact that the circumcised Jews are almost entirely free from this disease.

Even though the glans be covered with a long foreskin, if the individual attends to the daily cleansing of the balano-preputial sac there is no opportunity for irritating secretions to be retained; but we notice that cancer of the penis almost always occurs in men in the lower walks of life. of neglectful and uncleanly personal habits.

Any condition which gives rise to a balano-posthitis, such as the retention of decomposed smegma and urine under a phimotic foreskin, particularly if aggravated by a gouty diathesis in the patient, excites a persistent and long-continued irritation. In an elderly person in course of time the simple inflammatory process undergoes a transition into carcinoma of a polymorphous type, composed of large pavement-cells and small epithelial cells. 


\section{COURSE.}

No matter in what form the disease had its origin, its course is one of extension at the edges, accompanied by ulceration and breaking down in the older parts, and in most cases this is attended by the formation of large regetations. or fungosities, resembling a cauliflower in shape.

A thin fluid, of a most disgusting odor, which dries into scabs, is continually secreted.

As the cancerous process extends only by continuity, its adrance through the corpora cavernosa is not rapid, but the lymphatics readily take up the infections material, carry it to the glands in the grom, and these are usually involved quite early in the disease.

The inguinal lymphatic glands are often the seat of a mixed infection, if pyogenic bacteria have been conveyed to them through the lymphatics and canse them to become inflamed and suppurate.

\section{DIAGNOSIS.}

Every warty or papillomatous growth, or: persistent erosion? occurring on the glans penis, or inner surface of the prepure, in an elderly person, should always be regarded with grave suspicion.

It is often difficult to differentiate simple papillomata or a chronic balano-posthitis from carcinoma, but the age of the patient, the long, protracted duration of the sore, together with a base which is hard, infiltrated, and immovable, and an edge which is hard and infiltrated, would point strongly in the direction of epithelial carcinoma. The diagnosis conld be definitely determined by cutting a small piece from the growth and subjecting it to microscopic examination.

A gumma of the penis occurring in tertiary spphilis might be easily mistaken for epithelioma; but a few weeks treatment with mercury and iodides would cause the gumma to disappear.

\section{PROGNOSIS.}

The prognosis of epithelioma of the penis is, of course, fatal withont operation, and death occurs in from one to two years. If the disease is seen early and the growth removed by amputation of the penis and extirpation of the groin glands, the prognosis is good: but many cases come into the hands of the surgeon too late for a complete removal of all the foci of infection. Winiwarter reports 12 amputations, of which 5 remained permanently well, 1 died of 
the operation, and 6 had recurrences, 3 of which were in the stump and 3 in the glaurls.

\section{TREATMENT.}

As already indieated, complete removal of all deposits at the earliest possible moment oflers the patient the only opportunity of saring his life, and the application of caustics only excites greater activity in the growth and is a waste of valuable time.

Two forms of operation are in use, and a selection depends upon the extent to which the inguinal glands and corpora cavernosa are involved.

\section{OPERATIONS.}

Amputation of the Free Portion of the Penis.-Technique.-A No. 20 French sound is introduced through the meatus into the bladder to indicate the position of the urethra.

A harelip-pin is thrust through both corpora cavernosa, at the root of the penis, to hold in place a rubber band, which is made to eneircle the penis and act as a tourniquet.

The skin of the penis is then cut through with a eircular sweep of the knife, and turned back an inch. The corpora cavernosa are divided, down to the corpus spongiosum, which, with the urethra, is left to project like a spont for an inch, before being ent through.

The tourniquet is then unloosed and at least four arteries will require ligation. The skin-flaps are sutured together, and the urethra stitched to the margins of the slin-flaps.

A soft-rubber catheter is tied in the bladder to prevent the uriue from infecting the fresh wound.

Amputation of the Entire Penis.-This is a mueh more serious opreration than the former. but is demanded in the case of extensive infiltration of the corpora cavernosa with cancerous deposit.

Technique.-The pratient is placed in the lithotomy position, and a sound is introduced through the urethra into the bladder. An incision is made along the raphe of the scrotum, splitting it into two halves. The dissection is arried down so that the corpus spongiosum is seen perforating the triangular ligament, with the corpora carcrnosa lying on cither side and attached to the rami of the pubes.

The corpus spongiosum, containing the urethra, is then dissceted away from the corpora carernosa for three inches, cut through, and allowed to hang down out of the way, at the lower angle of the wound. 
The next step is to separate the corpora cavernosa from their attachments to the rami of the pubes. It is generally recommended that this should be done with a periosteal elerator. The elose attachment to the bones renders this a matter of considerable difficulty, and after separation there is a free hæmorrhage, which is difficult to control.

The author prefers to burn through the crura penis with a Paquelin cautery close to their attachments to the bone, and in this way the corpora cavernosa are readily freed from the pubes and without hæmorrhage. The Paquelin can also be used to stop bleeding, which generally occurs from the dorsal ressels of the penis under the symphysis pubis, at the upper angle of the wound.

The final steps of the operation consist in bringing the urethra up into the wound and stitching it to the margins of the skin-flaps, and then the skin on either side is brought into apposition and stitched.

A catheter may be carried through the urethra and left in the bladder to drain it.

In this operation the testicles are exposed and may be left in the wound, or castration may be performed, to quell the sexual desire on the part of the patient after his recorery.

The inguinal lymphatic glands should be remored at the time any operation is performed for the relief of malignant disease of the penis, for if the glands have become infected, which oceurs early in the disease, a recurrence of the cancer will inevitably take place later on. 


\section{DISEASES OF THE URETHRA AND ITS ADNEXA.}

\section{CHAPTER II.}

\section{ANATOMY OF THE URETHRA.}

THE urethra is a canal, open at both ends, whose walls lie in wrinkled folds and come closely in contact except when distended by the passage of urine or a catheter.

The length of the canal from meatus urinarius to sphincter resice is abont eight inches and it is divided into three regions:-

(n) The anterior or pendulous urethra, which is six inches in length.

(b) The membranous urethra, which is about one inch long.

(c) The prostatic, or posterior, urethra, which is one inch in length.

The anterior urethra is surrounded by the erectile tissue of the corpus spongiosum, which terminates in the bulb. At a point correpponding to the bulb. in the anterior urethra, and lying between the peno-scrotal junction and the anterior layer of the subpubic triangular liganent, for a distance of an inch, is a part of the canal which is termed the bulbous urethra.

The membranous urethra is the portion of the canal lying between the anterior and posterior layers of the triangular ligament. Its mucous nembrane is not so richly supplied with mucous glands ant follicles as the other portions of the urethra, and it acts as a barrier to the onward progress of a gonorrheal inflammation.

The membranous urethra is surrounded by bands of voluntary muscular fibres known as the eut-off muscle, or compressor urethre, which is normally in a state of tonie contraction, and acts as a valve to sabrate the anterior from the posterior urethra.

liefore the act of urination the cut-off muscle is relaxed in order to allow the urine to flow out past it, and by its voluntary contraction the flow of urine can be instantly shut off.

The cut-oil muscle is sometimes affected by a cramp-like contraction and fails to relax, causing retention of urine or rendering (16) 


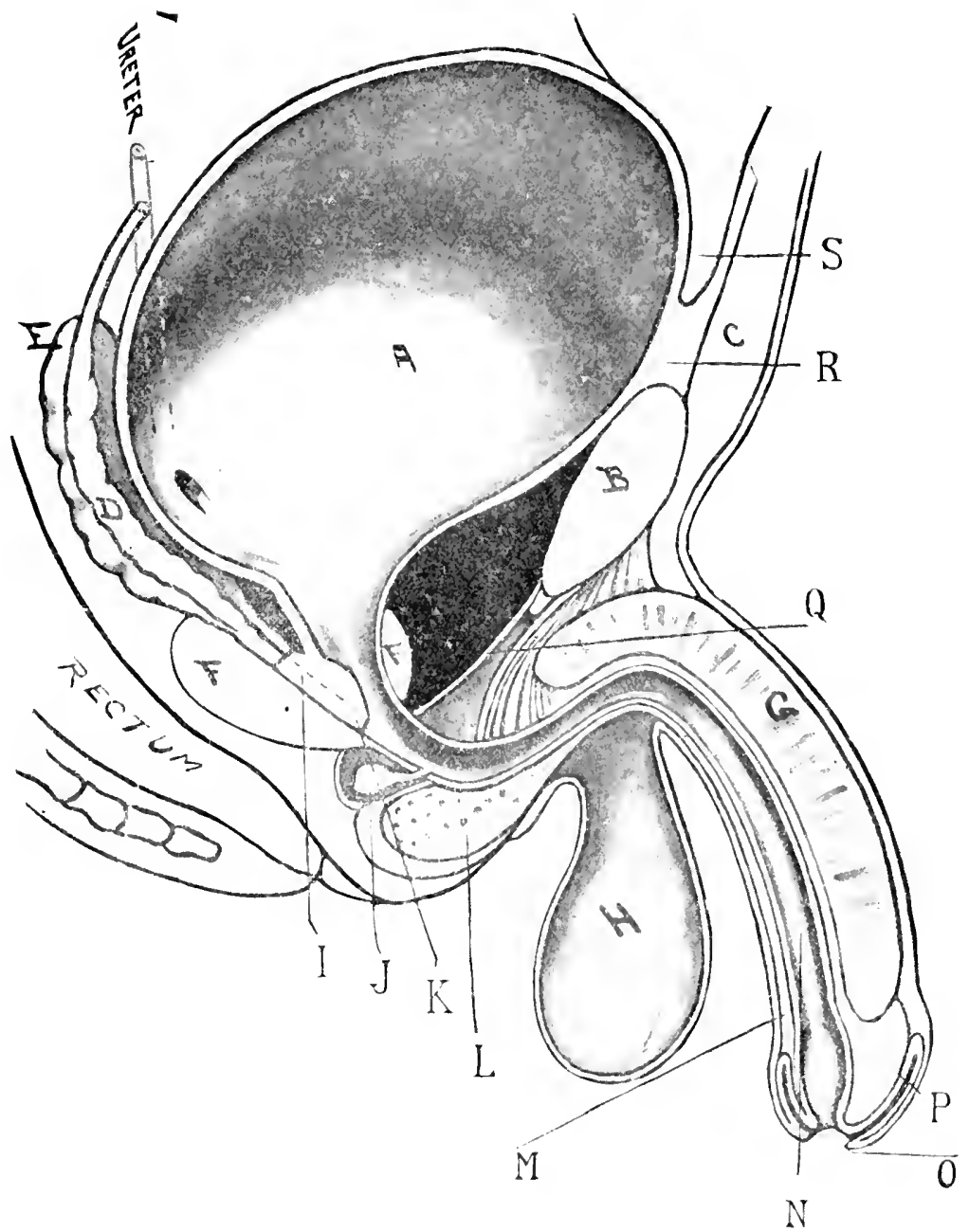

Fig. 2.-Diagram of Bladder and Urethra.

$A$, Interior of Bladder.

$B$, Symphysis Pubis.

$C$, Integument.

$D$, Vas Deferens.

$E$, Vesicula Seminales.

$F$, Prostate Gland.

G, Corpus Cavernosum.

$H$, Scrotum.

$I$, Verumontanum.
$J$, Cowper's Gland.

$K$, Orifice of Ejaculatory Duct.

$L$, Bulb of Corpus Spongiosum.

H, Corpus spongiosum.

$N$, Urethra.

(), Prepuce.

I', Glans Penis.

(2, suspensory Ligament.

$R$, space of lietzius.

$S$, Peritoneal Fold. 



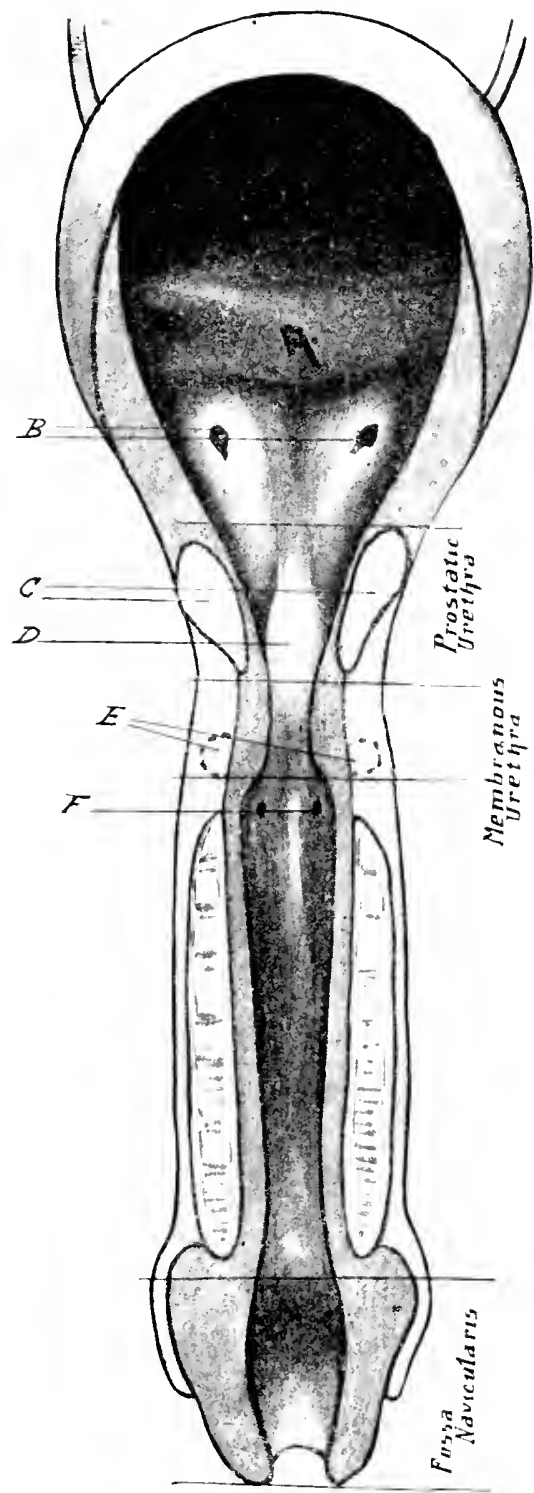

Fig. 3.-Diagram of Pladder and Uretlıra.

$\boldsymbol{A}$, Bladder Base.

$\boldsymbol{B}$, Opening of Ureters.

$C$, Prostate Gland.
L, Verumontanum.

L. Cowper's Glands.

$F$, Mouth of Ducts from Cowper's Glands 

it difficult or impossible to pass a sound. This condition is known as spasmodic stricture.

The prostatic, or posterior, urethra perforates the prostate gland. It is very richly supplied with mucous glands and folliches. Upon its floor is a small elevation composed of erectile tissue and abundantly supplied with nerves, and called the rerumontanum, or caput gallinaginis. At its base, in front, is a small depression, the sinus pocularis, or uterus masculinus. The ejaculatory ducts and the prostatic sinuses empty into the urethra on either side of it.

The urethra is not like a tube of uniform calibre, but is a canal of varying width and distensibility. There are three points of physiological narrowing:-

I. At the meatus.

II. Somewhere in the third inch.

III. The membranous urethra, lying between the anterior and posterior layers of the triangular ligament.

The points of widening which are susceptible of instrumental dilatation to a considerable extent are as follow:-

I. The fossa navicularis, which is located just within the meatus, and contains in its roof a large mucons erypt: the lacuna magna.

II. The bulbous urethra, which lies just in front of the triangular ligament and extends for one and one-half inches. It is the widest and most dilatable part of the anterior urethra.

III. The prostatic urethra, nsually termed the posterior urethra, is capable of greater distension than any other portion of the urethral canal, and may be dilated to 40 or 45 of the French scale without injury.

The mucous membrane which lines the urethra is soft, delicate, and easily lacerated, and is composed throughout its entire length, excepting the fossa navicularis, of epithelial cells of the cylindrical variety. It is richly supplied with glands and follieles.

The glands of Littré lie beneath the mucons membrane in the meshes of the corpus spongiosum and are true glands. lined with secreting epithelium and prorided with a duet which empties upon the free surface of the urethral mucons membrane. They are very numerous, and are arranged in groups together.

The follicles of Morgagni are simple crypts or depressions of the urethral mucons membrane, and are located unon the roof of the canal. 


\section{CHAPTER III.}

\section{ACUTE URETHRITIS.}

Necte urethritis may be divided into three varieties:-

(a) An aseptic catarrh, in which no micro-organisms are present and which is due entirely to chemical irritation.

(b) Simple urethritis, which is occasioned by inoculation of the urethral nucous membrane with progenic bacteria (staphylococci or streptococci).

(c) Specific or gonorrhæal urethritis, which is produced by the gonococens of Neisser.

Among the predisposing causes to any rariety of urethral inflammation may be mentioned a damaged condition of the urethra from previous disease which has left behind granulations, erosions, or a stricture; anatomical abnormalities, such as a long and narrow repuce, a wide urethral orifice, and hyposparias; certain diathetic states, such as gout. rhematism, and tuberculosis, which predispose to inflammation by lowering the resisting power of the body to bacterial invasion.

\section{SIMPLE URETHRITIS.}

Any of the pus-producing bacteria-i.e., staphylococcus, streptocorus, etc-will excite a catarrhal inflammation, with suppuration, of the mucons membrane. if introduced into the urethra. The microorganisus may be introduced from without, through sexual intercourse, and are contained in menstrual discharges, leucorrhea, suretions from an ulcerated cervix, and ntero-vaginal discharges of any character. They may also be introduced upon a dirty sound or atheter. On the other hand. the bacteria may lie dormant and monspected in the ratient's own urethra, concealed in the crypts and follicles or the folds of mucous membrane, and may be lighted in to activity througly orerindulgence in alcohol, intcnse erotic excitement, excessive coitus, or tramatism produced by the passage of a solnd. 
The inflammatory process in a simple urethritis is less serere than in the gonorrhœal form, and it is limited to the mucous membrane, without involving the submucous tissues.

\section{TREATMENT.}

The treatment consists in hygienic measurcs, rendering the urine bland, and the use of a mild astringent injection which answers very well for the light cases. If irrigations are used, the nitrate of silver ( 1 in 4000) frequently causes a prompt subsidence of the discharge, because the micro-organisms do not penetrate deeply into the tissues, but grow on the surface of the mucous membrane. Here the irrigation of nitrate of silver comes in direct contact with them and destroys them, and at the same time causes a desquamation of the superficial cells upon which the germs have proliferated. One or two irrigations may succeed in ridding the tissues of micro-organisms and infiltrated epithelial cells.

\section{GONORRHCEA.}

Gonorrheal inflammation of the urethra is cansed by the gonococcus of Neisser. The period of incubation is usually from four to seven days, but in rare instances may extend to fourteen days.

\section{PATHOLOGY.}

The gonococci are introduced into the urethra, usually in uteroraginal secretions or upon an infected instrument. They do not remain upon the free surface of the urethral mucous membrane, but rapidly penetrate between the epithelial cells, and are to be found in the submucous connective tissue itself. They increase and multiply in colonies, in the interepithelial spaces and upper layers of the submucous comnective tissue, and the toxic substances which they produce cause reaction on the part of the tissues, which is characterized by dilatation of the blood-ressels and discharge of serum and leucocytes. The cylindrical epithelium cells lining the urethra are loosened by the flow of secretion and are desquamated, forming erosions of the epithelial surface.

An active process of phagocytosis takes place, by which the 
gronoeoci are taken into the pus-cells and thus removed from the tissues, and, in cases which run a normal course, the gonococei have almost entirely disappeared from the submucous connective tissue and deeper layers of the mucous membrane by the end of the second or thind week.

When the stage of decline commences, the epithelial erosions begin to undergo repair, by being covered with squamous epithelium in many layers. The gonocoeci which have been removed from the deeper tissues now begin to grow luxuriantly on the free surface of the mucous membrane after the mamner of a sod of grass, and a desquamation of the upper layer of the newly-formed epithelial cells takes place, carrying with them their attached colonies of gonococci.

In normal and orelinary ases the process of getting rid of the

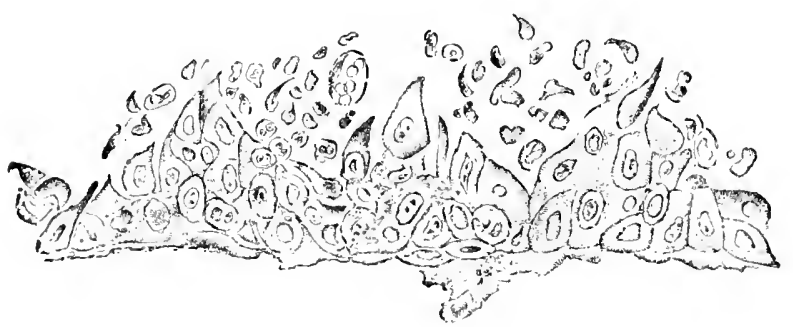

Fig. 4- Gonorrheal Conjunctivitis. Invasion of Epithelial Layer by Conococei, and Despuamation of the Sruperficial Layers of Cells.

gomorocer is accomplished in the ascending stage by phagocytosis, and in the stage of dectine throngh desquamation of the epithetial cells; so that in the fifth or sixth week, in favorable cases, the gonococci have disappeared entirely from the urethra, and the inflammation ceases.

Morgagni's crypts and Littré's glands are also affected by the inflimmmatory process, which oecurs within their cavities as well as aromed them, and the caritics of the glands act as foci of suppuration and incubating places for gonoroci for months after the inflammation has ecaled on the free surface of the mucous membrane. An Infiltration of small round cells which are derived from a proliferation of the fixed connectire-tissue cells, and from leucocytes which have escaped from the capillaries, occurs as part of the process of inflummation, and may be (a) superficial, or confined to the mucous 
membrane and its connective-tissne layer immediately underneath, and dipping down and surrounding Morgagni's crypts and Littrés glands, or may be the (b) deep form, in which, in addition to affecting the mucous membrane and glands, the small round-celled infiltration involves the deeper submucous tissues, extending into the spongy tissne of the corpus spongiosum, in severe cases. The erypts and follicles are usually surrounded by and imbedded in the small round-celled infiltration.

Healing of the lesions is brought about by a removal from the tissnes of the gonococci, throngh (a) phagocytosis, and (b) desquamation of the epithelial cells, which have been invaded by the gonococei. When the gonococci have been entirely remored suppuration ceases.

The erosions either (a) become covered with squamous epithelium in many layers, which offers a barrier to the renewed invasion of the tissues by gonococci, or $(b)$ the erosions remain without being covered by squamous epithelium, but become the seat of granulations, and occasion a gleety discharge.

The small round-celled infiltration (a) disappears by absorption, especially when supcrficial and confined to the mucous membrane, and the urethra becomes healthy, soft, and flexible, or (b) the small round-celled infiltration is not absorbed, especially when deep seated, -i.e., involving submucous tissue and carernous tissue of the corpus spongiosum,-but becomes transformed into true fibrous connective tissue, called stricture.

Relapses are frequent, and are due to reinfection of the submucous tissnes from a focus of suppuration located either in $(a)$ Morgagni's crypts or Littrés glands, (b) the seninal vesicles, or (c) Cowper's glands or the prostatic crypts.

The manner in which a relapse takes place is as follows: If premature coitus or an excess of beer is induiged in, hyperemia of the mucous membrane, with increased secretion, follows and the protecting layer of squamous epithelinm is torn asunder. The gonococci penetrate through these clefts into the deeper tissues and again cause their irritative symptoms.

If the gonococei remain in the urethra for a considerable length of time a state of tolerance of the tissues is establisherl and with each sucessive relapse the inflammatury reaction becomes less and less until, with the third or fourth relapse, the energy of reaction is not enough to bring the gonococei out of the submucons tissues. As a consequence, the gonococci remain and give rise to a perma- 
nent irritation of the submucons comnective tissue, an infiltration of smill round cells occurs, and the gonorrhoa becomes chronic.

\section{COURSE.}

The inflammation begins at the meatus, and in favorable eases allects the anterior urethri only, stopping at the cut-off musele.

Stages.-L'rodromal.- The symptoms are a slight tickling at the meatus, and a light-bluish sticky discharge with some slight stinging on urination. These last a couple of days, and then begins the increasing stage: The amomnt of pus increases. It is creamy yellow in color or greenish yellow from admixture with blood. Hæmorrhages may occur. The pain on urination is intense, and is oceasioned by the sudden distension of the infiltrated walls of the urethra by the outtlow of urine. Neuralgic pains in the back, perineum, groin, and spermatic cord are present. Constitutional disturbanee in the shape of fever and a feeling of prostration often occurs. The prepuce may become odematous and cause phimosis or paraphimosis.

Chordee is of frequent occurrence. It consists in an erection of the penis, with a painful incuration downward. It is caused by an infiltration into the spongy tissue of the corpus spongiosum which surrounds the urethra and renders it rigid and inextensible. On account of the rigidity of the urethra the stream becomes small and twisted, and dribbling after urination oceurs.

This condition continues without change for the better for about three weeks, and during this time it is known that the gonococei have been proliferating in the submucons tissues. At the moment when the gonococci have been removed from the deep tismes and begin to grow upon the free surface of the mucous memlrane, the acute symptoms subside, and usually in the course of the thind week the stage of decline begins. The pain on urination and the chordee lessen, the discharge becomes more watery and less in quantity, until it finally diminishes to a drop in the morning, which ultimately disappears.

Endoscopic examination, or the introduction of any instrument, should be strictly aroided in the acute stage. But if an endoscope were used, the appearances would be as follows:-

The mucous membrane appears swollen, addematous, intensely red, and covered with pus. The glands and follicles are very markedly affected. The mucous membrane around their openings 
is more swollen and the openings themselves gape. Erosions, from desquamation of the cylindrical epithelium, appear in the vicinity of the glands. Gramulations may form upon the erosions, later in the course of the disease. The submucous tissue is infiltrated, rendering the canal swollen and rigid.

Examination of Urine.--The two-glass urine test should be made at each visit to determine:-

(a) If the postcrior urethra has been affected.

(b) The amount of pus secreted.

The urine passed into a glass appears turbid from armisture with pus, and little clumps or mases of desquamated epithelium are present. After standing, the pus settles to the bottom of the glass, and a cloud of mucus appears floating above it. As the case goes on toward recovery the pus disappears, but the hypersecretion of mueus continues, and occasions a cloudy apjearance in the urine, resembling mucilage added to it.

After the mucus disappears clap-sheds persist for months, showing that isolated portions of mucous nembrane are not covercel with epithelium, and are still secreting pus.

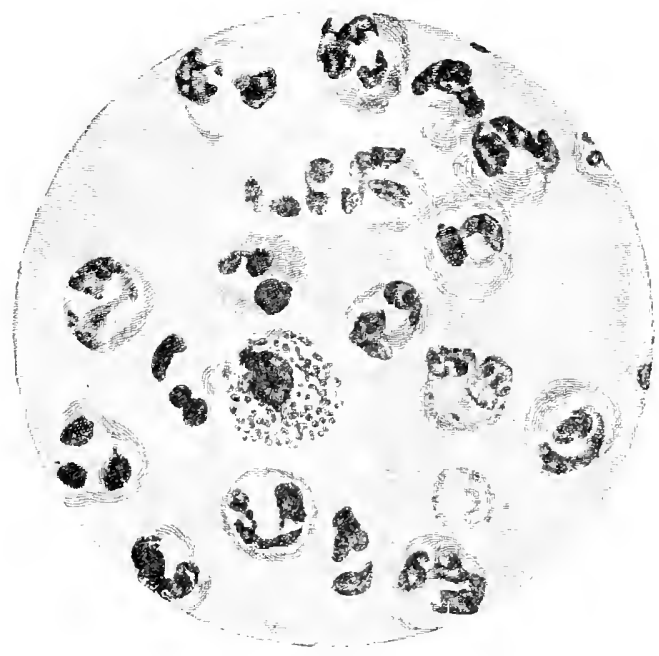

Fig. 5.- cionorrhueal Pus.

Microscopic Examination of Pus. - In the ascending stage numbers of pus-cells are present containing gonococci within the 
cell. In the stage of dectine a diminution is noted in the number of pus-cells. fewer gnocowi are present, and squamons epithelial cells make their appearance. Finally the pus-cells and gonoeocei disappear, and squamous epithelinm alone is found.

It is of the utmost importance to make sure of the entire disappearance of all gonococei before permitting coitus, on account of the danger of infection, in case the gonoeocei are not remored.

If no purulent discharge is obtainable from the meatus it is proper to excite a simple urethritis, by injecting the urethra with nitrate-of-silver solution gr. $x$ to the ounce.

If gonococei are lurking in the crypts or a granular pateh, the supluration caused by the injection will bring them to the surface, and they can be found hy microsenpic examination of the discharge.

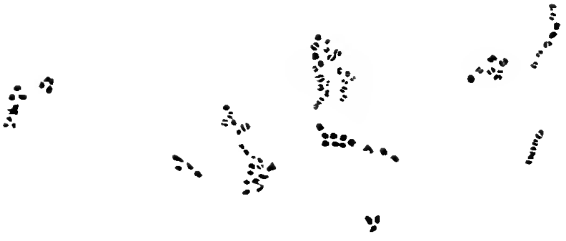

Fig. 6.-Gonococci Growing in Clusters.

\section{MORPHOLOGY OF THE GONOCOCCUS.}

The gonococeus resembles in appearance two eoffee-beans placed sirle to side, and, as it is removed by phagocytosis from the tissues, will of necessity be found lying within the pus-ecls. A few gonococei may be found ontside the pus-cells, if these have been ruptured. In the desending stage of gonorrhea the gonoeocci are found adherent to and growing upon the desquamated squamous epithelial cells.

Other micro-oreanisms--for example, the psendo-gonoeoceuswhich are not pathogenic resemble the gonococeus in shape, but it is believed to-day that the gonneocei can always be recognized by decolorizing them by Grants solution.

The microsopic examination for the gonococens is pursued as follows in the Hongland Laboratory, after the method of A. Hymans Tan den Bergit:--

I. Make a thin smear on a cover-glass of the pus supposed to contain the micro-organisms.

1I. Iry in the air and then fix over a flame. 
III. Stain in Löftler solution of methylene-blue (prepared by Eimer \& Amend) for $1 / 2$ to 1 minute.

IT. Wash in water, dry, mount in balsam, and examine.

The gonococcus is found on examination to be stained blue. In order to differentiate the gonoeocens from the psendo-gonococcus or other similar organisms, Gram's method of decolorization may be used, and if, after using it, the miero-organisms are found to have lost their blue color and become colorless, the diagnosis of gonococcus is established.

If, on the other hand, the micro-organisms still retain the blue color, it is a proof that they are not gonococci.

Gram Mcthod.-I. Stain in aniline-water gentian-riolet for one minute.

II. Pour off stain and wash or blot gently.

III. Place in Gram's solntion 1 minute.

IV. Decolorize in absolute alcohol $21 / 2$ to 3 minutes.

V. Dry and mount in balsam.

VI. Examine.

The gonococeus is found to be decolorized, but other microorganisms retain the blue color.

Formulæ.-To make aniline-water shake up 5 cubie centimetres of aniline-oil in 100 cubic centinietres of plain water. Shake violently, and filter through a wet filter.

Gentian-violet.-To 1 enbic centimetre of saturated alcoholic solution of gentian-violet add 20 cubic centimetres of aniline-water.

These added together make aniline-water gentian-violet, which does not keep, as a mixture, more than a week.

Gram's Solution.-Iodine crystals, 1 gramme; potassium iodide, 2 grammes; water, 300 cubic centimetres.

\section{DURATION OF AN ATTACK OF GONORRHEA.}

When the posterior urethra is not affected a favorable case of gonorrhœa recovers in six to eight weeks. In very exceptional instances recovery may oceur in three to four weeks, but in these cases there is always a doubt as to the correctness of the diagnosis of true speeifie gonorrhcea.

The first attack is the most severe, but most liable to recover withont stricture. Later attacks are apt to follow the course of the first one, in having a repetition of the complications. 
The causes which retard recovery may be grouped as follows:-

(a) Complications. posterior urethritis, prostatitis, etc.

(b) Reinfection from a urethral gland, seminal resicle, prostate, ete.

(c) Lack of rest.

(d) Habits of drinking.

(c) Injections which are too strong or too frequently repeated.

(f) Constitutional causes: i.e., gont, tuberculosis, etc.

(j) Premature coitus.

\section{TREATMENT.}

Gonorrhnea is a self-limited disease, and the suppuration may be looked upon as an effort on the part of the tissues to remove the invading micro-organisms; so that when the last gonococcus is removed suppuration ceases.

An expectant plan of treatment can only be carried out excepfionally, as in military hospitals, for instance. Here it is found that if a patient with gonorrhwa is put to bed and fed on a bland diet, consisting chiefly of milk, in forty-five days, on an average, the sonococci are eliminated from the tissues and the suppuration has ceased. Lnder the existing social and business conditions, however, such a plan of treatment is practically impossible, and we have to adopt the methodic treatment.

As the gonorrheal inflammation begins at the meatus and does not reach the posterior urethra until the third week, or in favorable cases not at all, we will first consider the treatment of inflammation which is limited to the anterior urethra, and take up posterior urethritis in a subsequent section.

\section{ANTERIOR URETHRITIS.}

Methodic Treatment.-There are certain hygienic directions which the patient should observe: he should keep quiet and spend as much time lying on a sofa or bed as possible, and should, of comre, aroir all sources of sexual or erotic excitement. He should be warned of the danger of gonorrheal ophthalmia, and directed to wash his hands after hamlling the penis or dressings, to avoid carrying any pus into the eyes.

The diet shonld be non-stimulating, and the patient should aroid meat in excess, highly seasoned or salty foods, sauces, condiments, strong tea or coffee, pickles, tomatoes, asparagus, and alco- 


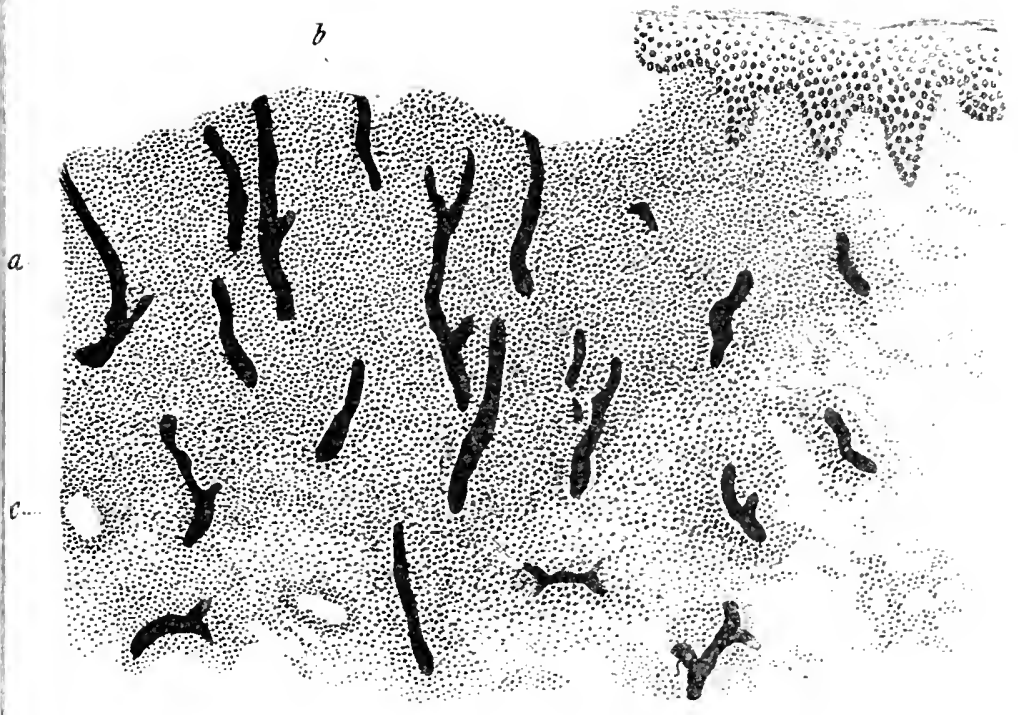

I. Section of Chaneroid.

$a$, Small round-celled infiltration.

$b$, Lymphatics, open and gaping.

$c$, Blood-vessel.

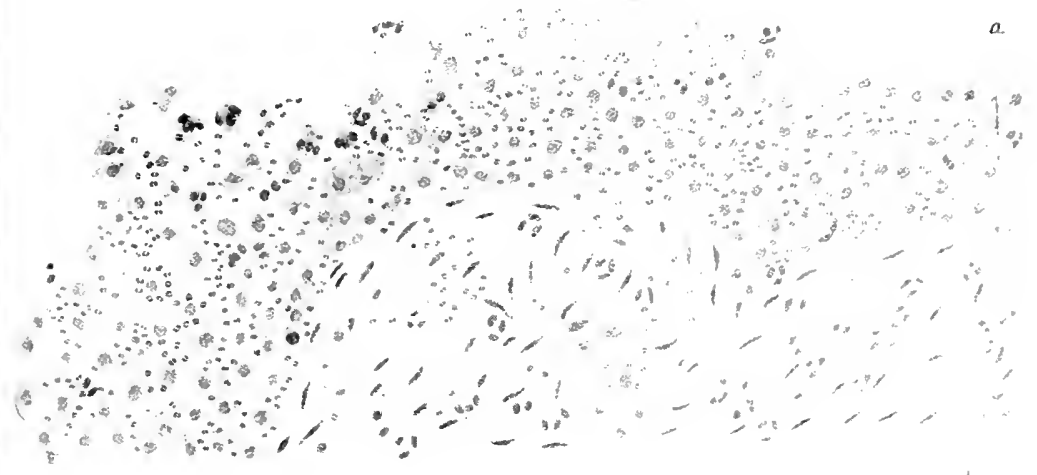

II. Acute Gonorrhma.

$a$, Cylindrical epithelium, infiltrated with pus-cells and gonococcl.

$b$, Submucous connective tissue, with pus-cells and gonococi. 

holic drinks of all kinds, of which beer and champagne are especially detrimental.

Dressings for the purpose of catching the discharge and keeping it from the clothing are always necessary. The best form is made by cutting off the foot of a stocking and placing some absorbent cotton at the bottom; the penis is placed within it and the bag suspended from a waist-band.

Constricting the penis by wrappings should be carefully avoided, so as not to interfere with the return-circulation. If the discharge is but trifling, a pledget of cotton may be placed under a long foreskin to absorb it; but the cotton is not to be recommended if the discharge is profuse, as it will prevent the pus from flowing out freely from the meatus, and cause it to dam back.

A suspensory bandage should be worn in every case to relieve the sensation of dragging on the spermatic cord and perhaps lessen the danger of epididymitis.

Therapeutic Treatment.-The balsams of copaiba, cubebs, and sandal-wood oil have had for years a well-deserved reputation as antiblennorrhagics. They are eliminated by the kidneys, and aflect the inflamed urethra as they pass over it, held in solution in the urine.

Sandal-wood oil is best adapted to the increasing stage, but acts well throughout the whole course of the disease. The balsams of copaiba and cubebs have fallen largely into disuse, but are sometimes serviceable in the descending stage of a gonorrhoea.

The dose of sandal-wood oil and the balsams is from 15 to 20 drops in capsules, three times a day.

Sandal-wood oil sometimes causes an intense pain in the back, or disagrees with the digestion, and has to be abandoned on these accounts, while copaiba often causes an erythematous eruption resembling measles.

While sandal-wood oil and the balsams are a useful adjuvant in gonorrhoa, it is necessary to have recourse to other measures to effect a cure.

Treatment of the Increasing Stage.-The bowels should be regulated by a saline cathartic given every second morning before breakfast, which depletes the pelvic blood-vessels and lessens congestion.

Altalies or an alhaline mineral water should not be prescribed as a routine measure, for an alkaline reaction of the patient's urine 
is always caused by his abstinence from meat and his free use of milk, and the best prophylactic against the development of cystitis in gonorrhea is a strongly acid reaction of the urine, which inhibits the growth of any bacteria which may find their way into the bladder.

l)iluents. - The patient should be directed to drink considerable quantities of pure distilled water-a glass every hour or two, with the object of washing out the urinary passages and keeping the urethra free from secretions which would otherwise form excellent culture-media for gonococci.

sandal-wood oil is administered by the mouth, preferably in capsules. The well-known Lafayette mixture is commonly used in hospital and dispensary practice, but it has the disadvantages of containing an alkali and of having an abominable taste. Its formula is as follows:-

R Extracti hyoscyamus fluidi............... f3ss.

Potassii citratis.....................

Olei santali flavi. ..................... f

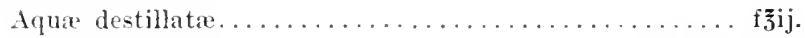

Syrupi acacia..................... s. ad f $\tilde{z} v j$.

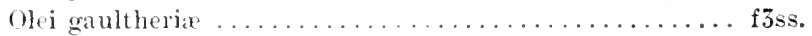

M. Sig.: Two teasponfuls at a dise.

The burning on urination is lessened in some degree by the sandal-mood oil, but if very severe can be relieved by injecting 5 ss of t-per-cent. cocaine solution into the urethra.

The local treatment by means of astringent injections is entirely contra-indicated during the ascending stage, for, as we have already noted in considering the pathology, the gonococei at this time are in the upper layers of the submucous connective tissue and the deeper cells of the mucous membrane, and they are heing removed from the deeper tissues as rapidly as possible by the phagocytic action of the leucocytes. Cnder these conditions the action of an astringent is to hinder the elimination of the gonococei from the depths, and so retard the natural healing process.

A puro antiseptic, howerer,- - such as some of the new silver salts or irrigations of pemanganate of potash by the Janet method, -is entirely in orter, and is effectual in moderating the severity and shortening the duration of the disease.

Treatment of the Stage of Decline.-In the third week the ascending stage has nsually passed its acme, the chordee lessens, and 
the pain on urination is diminished. The character of the discharge also changes. It is no longer thick yellow or greenish pus, but is thinner, contains more mucus, and is whiter and more watery in color. When these conditions oceur, the administration of sandalwood oil can be stopped and balsam copraiba, or oleoresin of cubebs substituted.

The use of astringent injections should be strictly avoided until the stage of decline, for the reason that, as stated before, in the ascending stage the gonococei are in the deeper larers of the mucous membrane and subepithelial tissues, and they cannot be destroyed, at this time, by the use of astringents, which also act as chemical irritants and interfere with phagocytosis.

The improvement in the symptoms of the patient is cansed by the fact that in the stage of decline the gonococei have been eliminated from the deep tissnes and are now growing on the free surface of the mucous membrane of the urethra, and the erosions are being healed by the formation of squamons epithelinm.

These facts can be demonstrated by finding, with the microscope, colonies of gonococei growing on desquamated cells of epithelium, which are contained in the discharge.

Under these conditions a combination of an astringent and an antiseptic as an injection is called for, in order to destroy the gonococci, heal the erosions. and contract the dilated blood-ressels.

Syringes are of various shapes and made of soft rubber, hard rubber, and glass. An important point to observe is that the nozzle is not prolonged into a snout, which would irritate the mucons membrane of the fossa navicularis. In addition, it is essential that the syringe should hold from three to four drachms of flnid, so that when the injection is given the folds of the urethra may be fully distended.

Technique of Injecting.-The patient urinates to cleanse the urethra, and then, holding the penis in the left hand, draws it out, while with the right hand the injection is slowly forced from the syringe into the urethra and held for two to five minutes. It is not necessiry to make pressure on the perineum to keep the fluin from entering the bladder, as the fluid is kept from flowing backward by the tonic contraction of the cut-off mucke.

Formulæ for Astringent Injections.--

Pe Zinci sulphatis gr. i-v.

Aqua . f.̃̄j. 


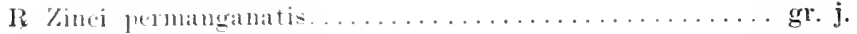

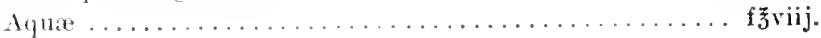

R Argenti nitratis. . . . . . . . . . . . . .

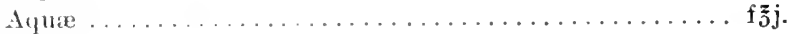

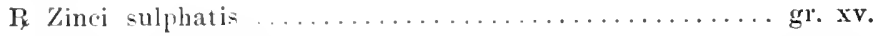

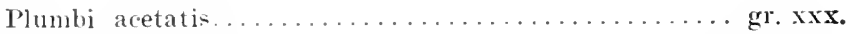

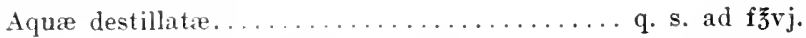

M. Sig.: Insoluble. Hold in urethra and let out drop by drop.

R Extracti lydrastis fluidi (non-alcoholic) .......... f3ss-j. Aqua .................... $\mathrm{f} \tilde{z} \mathrm{j}$.

M. Sig.: For muco-purulent discharge where simple astringent is indicated.

\section{L7trmann's Injection.-}

R Zinci sulphatis........................ gr. ivij.

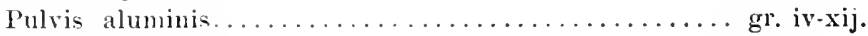

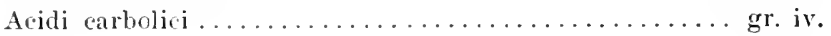

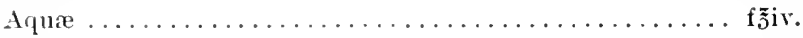

The above is particularly useful in the stage of decline where discharge fails to diminish under other applications.

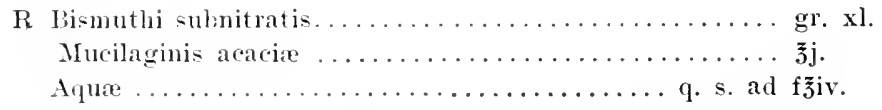

M. Sig.: Shake well. Use once a day at bed-time and hold in urethra five minutes.

The Use of Injections.-An astringent injection should never be uscd in the presence of posterior urcthritis nor in the ascending slage of a gonorrhoa, for until the stage of decline sets in the gonocoeci lie deep within the tissues and entirely out of reach of astringents applied to the surface of the mucous membrane. In addition to being of no value in destroying the gonococci, astringents are actually hamful from the irritation of the tissues which their use cntails.

In the stage of decline. however, when the burning on mrination is decreased and the discharge has become thin and watery, the gonococei are growing upon the free surface of the mucous membrane and can be destroyed by the application of the various injections which combine an antiseptic and astringent. At the same time 
the dilated blood-vessels are contracted by the astringent and the inflammation, through their ageney, is lessened.

An injection should never cause more than a slight burning. After a time the urethra becomes tolerant of one form of injection, and it is necessary to increase its strength or to change the formula.

The prolonged use of an injection may in itself induee an irritation of the mucons membrane of the urethra. which is evidenced by shreds in the urine, and the formation of a slight amount of secretion, which causes the lips of the meatus to stick together.

In order to determine if the treatment is responsible for keeping up the diseharge, it is desirable to stop the use of the injection for forty-eight hours, to see if the last traces of inflammation will not disappear spontaneously.

If at the end of this time the discharge still persists in small quantity and on microseopic examination the shreds are found to be made up of squamous epithelium and contain no gonococei, an injection of bismuth used at night, which acts mechanieally by coating over the mueous membrane, frequently causes a cessation of the catarrh in six or eight days.

The patient, however, cannot be considered enred until he has resumed his ordinary way of life for some days and remained free from a relapse, because a few gonococci may have lain unsuspected in a crypt or follicle and on slight provocation come to the surface and cause a reinfection.

The abortive treatment of gonorrhœa, by means of strong solutions of nitrate of silver, injected into the urethra or applied through an endoscope, is not to be recommended. The miero-organisms lie deep in the tissues, and cannot be reached by applications made upon the surface of the mucous membrane, and an increased irritation of the tissues is sure to follow, without any shortening of the course of the disease.

Irrigations with Permanganate of Potash: Janet's Method.--In the last few years the treatment of gonorrhcea, suggested by Janet, of Paris by means of irrigations of permanganate-of-potash solution, has been extensively used. Its adherents are enthusiastic in praise of its merits, Goldberg. of Cologne, eiting statisties showing that 90 per cent. of the cases of gonorrhea were cured in fourteen days by this means.

The permanganate irrigation is a valuable method of treating gonorrhoca, but these claims as to its advantages would seem to be 
extravagant. According to the experience of other men who use this form of treatment. it is impossible to attain any snch results, and many specialists have giren np the method in disgust, after a fair trial.

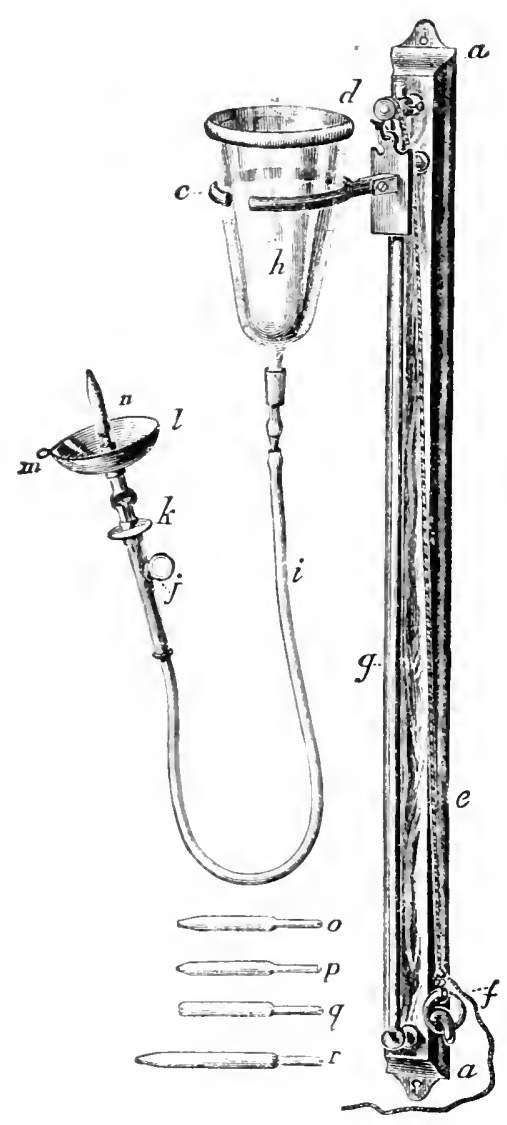

Fig. T.-Talentines- Irrigitor.

From the author's reading and experience with the Janet method, the following would seem to be a fair estimate of its ralue:-

The profuse purulent discharge is checked, in most cases, in about eight days: but eren under treatment relapses, accompanied by a free discharge of mos, nccur in nearly every case, without apparent cause, and often several times. These relapses yueld 
promptly to irrigations of permanganate, but the convalescence is retarded, and the irrigations have to be continued daily to holl the pus in cheek and to control the thin. serous discharge which lasts after suppuration ceases. The course of the case is in this way dragged out; so that at least one month, and very often two months or more, are required to effeet a cure.

The adrantages of the method are that posterior urethritis is of exceptional oceurrence. The discharge from the meatus is so slight as not to cause any inconvenience, and there is no burning on urination and no chordee.

The disadvantages of the Janet method are the expense, trouble, and inconvenience entailed upon the patient by being obliged to report at his physician's office once or twice a day for treatment.

The objection which has been raised, that the frequent irrigation of the bladder or the relaxation of the cut-off muscle wll in time do harm, is, I believe, entirely unfounded.

In order to ent short the course of the gonorrhoea by Janet's method it is necessary to begin treatment early in the ascending stage.

Technique.-The patient sits easily, well forward on a chair, and rests his back against the back of the chair. The irrigator nozzle is inserted into the meatus and the anterior urethra washed out. If it is desired that the fluid should enter the bladder, the irrigator is elevated to the height of nine feet from the floor. The patient is instructed to take a long breath and attempt to urinate. As soon as the ent-off muscle is relaxed the solution from the irrigator flows into the bladder, and when the bladder is filled the patient stands up and urinates, and the solution flows out through the urethra.

The urethra is irrigated twice a day for the first week, and then once a day till the patient is cured. The first irrigation is used of a strength of 1 to 1000 for its abortive effect, in the anterior urethra alone, and the second time of a strength of 1 to 6000. From this time on the strength of the solution should be 1 to 3000 .

As a rule, the first irrigation diminishes the secretion materially, and when after a few days the secretion is scanty in amount and thin the irrigation is allowed to flow through the posterior urethra into the bladder.

After the first week one irrigation a day is sufficient, and the 
strength may be increased to 1 in 1500 , mnless it canses tenesmus and Madder irritation.

The effect of permanganate irrigations is to cause energetic disinfection of the nucons membrane by removing and mechanically washing away accumulated secretions, as is the case with the irrigation of any suppurating wound-cavity. The permanganate also canses an cedematons swelling of the epithelial cells, which inhibits the growth of colonies of bacteria.

It has been suggested recently that, after the discharge is reduced. at the end of the first week, the destruction of gonococei may be lastened by allowing the patient to use Protargol or Largin as an injection, and when the case has progressed so far that there is no more pus-formation, but only a slight serous discharge and shreds in the urine, an astringent injection may complete the eure.

The Salts of Silver.-In the last comple of years various combinations of silver with an albuminoid base, such as Protargol, Largin, Argonin, and Argentamin, have been introduced for the treatment of gonorrhoa.

According to Finger, ${ }^{1}$ Protargol and Largin may be regarded as pure antisepties withont astringent properties and entirely unirritating to the tissues; so that they can be used in the ascending stage. They are in the form of a soluble albuminate of silver, which does not coagulate the tissues, and has the power to penetrate deeply and destroy the gonococei which lie under the mucous membrane.

The action of the silver salts is incomplete, and they do not destroy all the gonococi, particularly those in the mucous crypts and follicles; so that if their use is discontinued too soon a relapse is liable to ocenr.

It has been foumd experimentally that Protargol and Largin must remain in contact with the tissues for 10 minutes, in order to exert their destructive antion upon the gonococei. A shorter time is insuflicient. and fails.

Another adrantage possessed by Protargol and Largin is that, in ases where the inflammation has passed beyond the ent-off musele and attackul the posterior mrethra, on holding a solution of either drug in the urethra for a sloort time the cut-off muscle relaxes. on account of the mill and unirritating character of the remedies, and

Winel Klinik, January, lomo. 
allows the solution to flow back and come in contact with the posterior urethra and exercise its bactericidal power.

If a strong and irritating solution, like nitrate of silver, is injected into the anterior urethra, a reflex contraction of the eut-off muscle is always caused.

In the ascending stage of gonorrhea Protargol and Largin are the most useful of the silver salts; but in the stage of decline the gonococei are no longer in the deep tissues, but superficially seated. The indication at this time is to destroy them on the surface of the mucous membrane and at the same time exert an astringent effeet upon the dilated vessels. Argonin and Argentamin are astringents in addition to being germicides, and are indicated in this stage.

Method of Using.-The use of Protargol solution $\left(1 /{ }_{4}\right.$ to 1 per cent.) should be begun at once in the ascending stage, and the patient should be instructed to make the injections eight hours apart. This is said to be an important point. Before using the injection he should urinate, and, as urine decomposes the silver solutions, he should wash out the urethra with 2 or 3 syringefuls of warm water. The injection should be warmed before using, the syringe should contain 3 to 4 drachms in order to distend the folds of the urethra, and the solution should be retained in the urethra from ten to fifteen minutes.

In the course of a few days the acute symptoms subside and the pain on urination and chordee disappear. It should be noted that, if treatment be discontinued at this point, even though the discharge has ceased and only a few shreds remain in the urine, a relapse is certain to occur in from two to three weeks, for a few gonococei have been left which were not destroyed, and reinfection oceurs.

With the subsidence of the aeute symptoms the strength of the Protargol solution may be increased to $1 / 2$ or 1 per cent, and it should be used three or four times a day.

After Protargol has been continued a few days longer it is desirable to discontinue its use and begin with Largin solutions, commencing with $1 / 4$-per-cent. solutions and increasing to $1 / 2$ and 1 per cent., three times a day.

It is proven that, while Largin is not a neutral solution and has slightly irritating qualities, it poscesses the power of penetrating more deeply than Protargol, and destroying the gonococci lying in the deep tissues. 
After a few days more of treatment the discharge ceases entirely, but few slueds are present, and the gonococei are only found in very small numbers in the secretions; but, if the injections are stopped, the gonococei still remaining increase in numbers, and cause a relapse.

The indications at this time are to destroy the few remaining gonocoeci and to eure the eatarrh of the mucous membrane.

This is accomplished by the combined use of antiseptics and astringents, and to that end 1-per-eent. Largin solution may be used once a day, and Cltzmann's injection of zine, alum, and carbolic acid twice a day.

Later on, when the number of shreds is very much reduced, Largin may be used twice a day and either nitrate of silver or Argentamin solution $(1 / 4$ or $1 / 2$ of 1 per cent.) may be injected once daily.

A great advantage of the treatment with silver salts. which may also be assisted by the internal use of sandal-wood oil, is that the disinfection of the tissues adrances rapidly and the spread of the inflammation is checked; so that, while under ordinary treatment posterior urethritis occurs in 80 per cent. of the eases. in the patients treated with silver salts posterior urethritis is observed in from only 30 to 40 per cent. of eases.

In point of time, while a small pereentage of cases are cured in from two to three weeks, under the treatment with silver salts, in the great majority of patients in which complieations do not occur a continuous course of medication of five to six weeks is requiled in order to effect a cure. 


\section{CHAPTER IV.}

\section{POSTERIOR URETHRITIS.}

Postenion urethritis consists in an inflammation of the mucous membrane of the posterior urethra, which lies behind the cut-off muscle. In severe eases the inflammation may extend up out of the urethra and involve the base of the bladder.

Acute posterior urethritis is almost always caused by the extension of a gonorrhœal or simple urethritis from the anterior part of the canal; but the subacute and chronic forms may be the result of prolonged congestion from sexual abuses, complieated by germ infection from the rectum or from withont.

Posterior urethritis is a serious complication of gonorrhcea, because

I. It increases the extent of the inflamed surface and renders recovery more remote.

II. On account of complications, which are almost sure to follow, if the pus is conveyed through the rarious duets opening into the posterior urethra, to the prostate, seminal vesicles. testicles, and bladder.

Posterior urethritis oceurs in 80 per cent. of cases of gonorrhcea, but is often so mild as to be overlooked. It usually develops from the second to the fourth week, or when the gonorrhoal inflammation has extended to the bulb. It may be excited by the use of a sound or catheter in an acutely inflamed urethra, which pushes the pus along in front of the instrument or causes tranmatism, or it may develop spontaneously.

The membranous urethra, in certain eases, acts as a barrier to the spread of an inflammation from the anterior urethra backward toward the bladder. The ent-off muscle, which surrounds the membranous urethra, is in a state of tonic contraction, and acts like a valve, and the mucous membrane lining the membranous urethra is less vaseular and provided with fewer erypts and follieles than either the anterior or posterior urethra, and also serves to check the extension of the gonorihea.

Clinically two forms of posterior urethritis are met with: mild or subacute form; (b) severe form. 


\section{SYMPTOMS.}

The symptoms of the mild form may be so slight as not to attract attention. There is an ill-defined sense of weight over the pubes and a feeling of pressure in the perineum, together with slightly inereased frequency of urination.

The symptom of increased frequeney of urination depends upon the fact that, in a condition of health, the posterior urethra is the most sensitive part of the canal, and, when the bladder becomes filled and a few drops of urine trickle into the posterior urethra, their presence sets up a certain physiological irritation, which is transmitted to the bladder, and produces a contraction of its muscular walls, which empties the bladder of its contained urine.

In posterior urethritis there is a congested state of the prostatic urethra and an abnormally acute sensitiveness; so that the stimulus to urinate is greatly increased.

In the severe form of posterior urethritis the symptom of frequent and painful urination is very much aggravated. If the inflammation is very acute and particularly if it has extended to the base of the bladder, violent vesical tenesmus sets in, which the patient camnot restrain.

'The squeezing together of the inflamed surfaces, by the muscular contractions of the bladder, not only canses intense pain, but also often ruptures some of the eapillaries in the mucous membrane; so that a few drops of blood generally follow the act of urination.

No sooner has one spasmodic eontraction of the bladder subsided than another one sets in, and these continue to recur every few minutes until the suflering becomes almost unbearable.

\section{DIAGNOSIS.}

The diagnosis of the severe form of posterior urethritis can usually be made from the syniptoms alone, but the mild form is apt to be orriogked, unless we direct our patient with gonorrhoea to urinate in two glasses, each day we see him, and in this way we can detect intlammation of the posterinr urethra in its incipiency.

The two-glass urine test, as derised by Sir Henry Thompson, is based upon the physiological attion of the cut-off musele, which, by its tonic contraction, forms a barrier between the anterior and posterior urethra. Fluids injected into the anterior urethra cannot flow back into the bladder, and pus lying in the posterior urethra is prerented fron flowing out through the anterior urethra, but eseapes 
backward into the bladder, and renders the urine which had acemulated in the bladder turbid and cloudy.

If suppuration is going on in the anterior urethra (the posterior urethra being healthy), and the patient is direeted to urinate, the first gush of urine washes ont the pus, and the urine, if caught in a glass, appears turbid. If the remainder of the urine, which had collected in the bladder and is uncontaminated, is passed into another glass, the urine in the second glass is clear.

If suppuration is present in both anterior and posterior regions of the urethra, the first glass of urine passed is, of course, turbid, from the pus washed out of the urethra, and the second glass will be turbid also, because the pus formed in the posterior urethra flowed back and stained the urine which was contained in the bladder.

Turbidity of the urine is sometimes caused by urates or phosphates. If uratic in origin, the cloudiness clears up on boiling, and, if phosphatic in character, a few drops of nitric or acetie acid will render it clear. The presence of pus ean be determined by microscopic examination or by adding a few drops of liquor potassa to the suspected urine, in a test-tube, and twirling it rapidly. If pus is present it will be coagulated and float in long, ropy strings through the urine.

Chemical examination shows more albumin than can be accounted for by the pus. This superabundance of albumin is not oceasioned by structural changes in the kidney, but to increased intrapelvic pressure, caused by the frequent and severe muscular spasms of the bladder.

\section{TREATMENT OF THE MILD FORM OF POSTERIOR URETHRITIS.}

The irrigation of the urethra and the bladder by means of an irrigator, after Janet's method, is particularly adapted to cases of inflammation of the posterior urethra developing in the course of a gonorrhœa. Irrigation of the posterior urethra can also be praeticed by introducing a soft-rubber catheter beyond the cut-off muscle so that its eye lies in the posterior urethra, and injecting solutions through it by means of a large hard-rubber syringe. The best solutions to use are: Nitrate of silver, 1 in 4000 to 1 in 2000, or permanganate of potash, 1 in 6000 to 1 in 1500 ; and both, of course, should be used hot.

Instead of using copious flushings of the urethra, with a considerable quantity of fluid, we can deposit a few drops of a concen- 
trated solution of nitrate of silver directly upon the surface of the mucous membrane by means of Ultzmann's syringe. The syringe is introduced past the cut-off muscle, so that the end lies in the posterior urethra, and 15 or 20 drops of the solution are injected. Nitrate of silver, in strength ranging from 1 to 5 grains, is the best application for the purpose.

If we desire to medicate the posterior urethra alone, the patient should retain some urine in the bladder. The urine will neutralize the solution as it flows into the bladder. If a urethro-cystitis be present, the patient shonld empty his bladder first, and the injected fluid will then medicate the posterior urethra and flow back and aflect the base of the bladder as well.

As to a selection between the methods of irrigation and instillation, as a general rule, it is found that recent cases with an abundant purulent seeretion and which are free from pain or other aeute symptoms are most benefited by copious irrigations, and that after the discharge has diminished, so that the urine is elear and only shreds are present, instillations are more serviceable.

Diuretics-such as triticum repens, ura ursi, buchu, etc--are not indicated in posterior nrethritis, since, although they render the urine bland and unirritating, they increase the quantity secreted, and occasion more frequent calls on the bladder to empty itself.

\section{TREATMENT OF THE SEVERE FORM OF POSTERIOR URETHRITIS.}

In this form any kind of mechanical interference with the urethra-such as injections, irrigations, or the introduction of any instrument-should be riqully aroided. The patient should go to bed in order to secure rest for the inflamed posterior urethra, lessen its congested condition, and so diminish the resical tenesmus.

A mild saline cathartic is useful by reducing the congestion of the pelvic organs. Milk shonld be the staple article of diet, to render the urine bland and unirritating. Sandal-mood oil acts almost like a specific in some cases. After a few doses the tenesmus lessens and the escape of blood after urination ceases.

Alhalies or allialine mineral waters are contra-indicated, for the reason that the urine in the bladder is neessarily neutral or alkaline in reaction, on account of the abstinence from meat and the milk diet ordered. If the urine beeomes alkaline and pyogenie miero-organisms make their way into the blaller from without, a suppurative cystitis 
is almost sure to occur; so that a moderate degree of acidity of the urine is regarded as the best prophylactic against cystitis.

In order to maintain this condition of acid urine, it has been adrised of late to administer salicylate of soda, gr. xx three times a day, as this drug has the property of causing a strong acid reaction in the urine.

Morphia is generally required to relieve the excessive tenesmus and allay the frequent desire to urinate. The frequent desire for urination has a bad effect upon the inflammation, since the contractions of the bladder cause an increase in the hyperamia at its base. Morphia may be given by the mouth, but preferably in suppository.

Hot sitz-baths prolonged for half an hour and used several times a day sometimes lessen the tenesmus and desire to urinate;

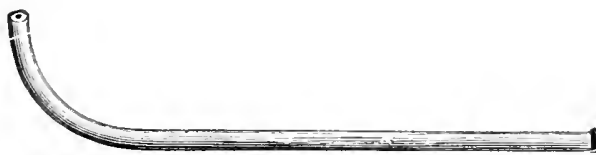

Fig. 8.-Ultzmann's syringe.

but, while useful as an adjurant, they will hardly take the place of opium.

When these methods fail to relieve the tenesmus and pain, which may be intense, the instillation of 10 drops of nitrate of silver with an Cltzmann syringe into the posterior urethra often succeeds in calming the disturbance in a few hours. We begin witl gr. $j$ to the ounce and inclease up to gr. $\mathrm{v}$ to the ounce, using it erery second or third day.

It is always better to use the instillation as a last rosort, since we can lay it down as a rule from which there are few departures: "Never to introduce an instrument into a uretlira affected with acute inflammation."

After acute symptoms have passel off the case assumes the characteristics of the mith form of posterior urethritis, and can be treated as such. 


\section{CHAPTER V.}

\section{CHRONIC URETHRITIS.}

Chrovic urethritis is one of the most obstinate and difficult affections to cure which the genito-urinary specialist is called upon to treat, unless the treatment is based upon a knowledge of the pathological changes which have taken place in the tissues, and the character and exact location in the urethra of the lesions.

A case of gonorrhoea may be called chronic when it has lasted for ten or twelve weeks.

Chronic urethritis is sometimes incorrectly called "glect," but the true definition of gleet is: A chronic muco-purulent discharge produced in certain localized areas of the mucous membrane of the urethra which are in a state of chronic catarhal or granular inflammation.

The statement that every case of gleet is dependent upon a stricture is an incorrect one. A gleety discharge may be occasioned by superficial changes in the mucous membrane, which never produce any narrowing of the urethra. On the other hand, in cases where stricture does exist, the mucous membrane lying above is never healthy, and a gleety discharge is always present.

The predisposing causes of chronic urethritis may be summed up as follows: Anything which tends to prolong or prevent the natural healing of a gonorrhœa, such as: (a) Careless ways of living on the part of the patient. (b) Injections which are too strong or used too frequently. (c) Use of alcohol or beer. (d) Sexual intercourse or erotic excitement.

At other times cases which are properly treated and which have had good care become chronic, usually on account of some diathetic taint, either tuberculosis, rheumatism, gout, or sometimes incipient pulmonary phthisis.

It is convenient to study chronic inflammation of the anterior urethra by itself, although the posterior urethra is apt to be affected as well, at the same time. 


\section{CHRONIC ANTERIOR URETHRITIS.}

\section{PATHOLOGY.}

- As a result of gonorrhwa. particularly in its later stages, an infiltration of small round cells takes place. This infiltration is the most important characteristic of the disease, and most of the other changes in the tissues result from it. The small round cells originate partly from the capillary vessels of the mucosa and partly from a proliferation of the fixed comnective-tisuse cells.

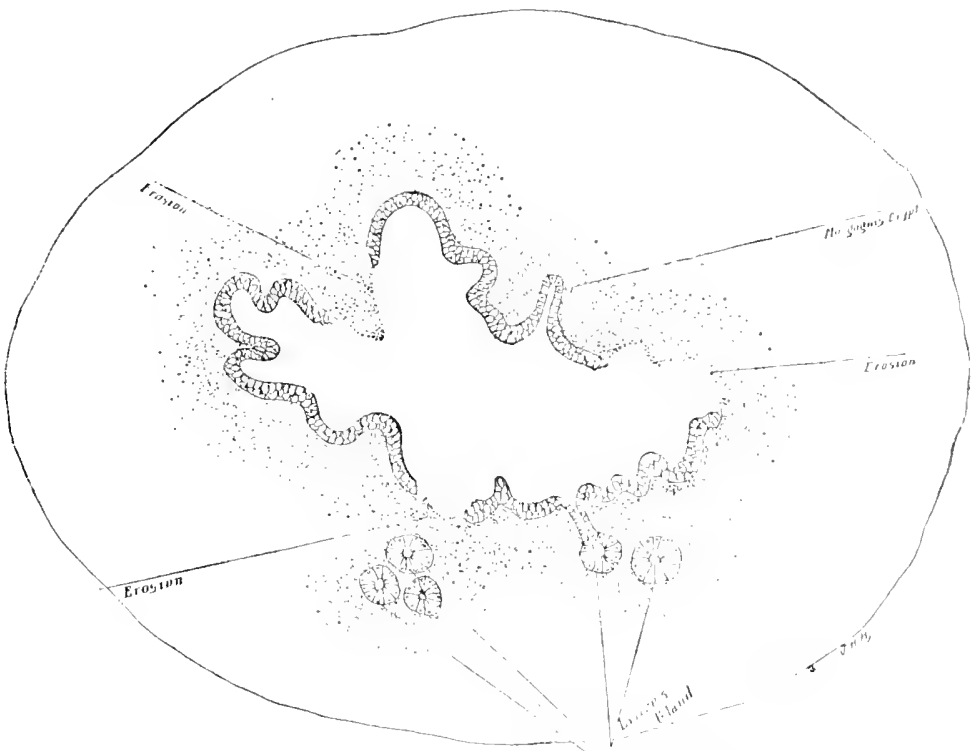

Fig. 9.-Diagram of a Cross-section of the Urethra, Representing the Histological Changes in Chronic Urethritis.

The small round-celled infiltration begins in the submucous connective tissue and surrounds the lumen of the mrethra completely.

Its favorite points of location are most freguently around the bulbous urethra, and next in frequeney at the fossa naviculario. These are the widest and most dilatahle portions of the canal, and in acute gonorrhea the stream of urine is not sufficient to wash ont the pus, which stagnates here and acts as a focus for the renewed local infection of the tissues at these points. 
'The mutous glands and follicles (Littré's glands and Morgagni's erypts), which dip down into the meshes of the corpus spongiosum, are also surromded by and imbedded in the infiltration.

Clinically we may classify the cases of chronic urethritis according to the extent of the infiltration into: (a) superficial, or mucons, form, in which the small round-celled infiltration is confined to the mucous membrane, subepithelial connective tissue, and periglandular tissue alone, and which is not followed by stricture, and (b) the deep form of infiltration.

In the latter class the infiltration extends deeply into the tissues, and involves the meshes of the corpus spongiosum extensively and is always followed by stricture.

Glandular Clanges.-During the course of the gonorrhea the gonococci penetrate into Morgagni's crypts and Littrés glands and set up an inflammation in the cavities, which is accompanied by an infiltration of small round cells around the ducts and walls of the glands (periglandular infiltration).

The infiltration around the duct stiffens it and keeps its mouth open and gaping, affording an open gateway for the escape of the inflammatory products which have formed within the cavity of the gland.

The gonococei may continne to propagate within the cavities of the glands for months, after the inflammation has entirely ceased on the free surface of the mucous membrane.

The reappearance of an acute purulent discharge containing gonococci, a so-called relapse, is due to an escape of gonococei from the plands and a reinfection of the surface of the mucous membrane.

Infection of wives and mistresses with gonorrhœa, during coitus, is often callsed in the same way, viz.: the escape of pus-cells containing gonococi, which had been formed by the suppurative process continuing in the gland-cavities after the surface of the mucous membrane had been well for months. During intercourse the pus, mixed with s'minal fluid, is deposited in the vagina of the female, and infection follows.

The inflammatory products, consisting of desquamated epithelial cells, pns-cells, and granular material, which stuff the cavities of the glands, are mashed out by the act of urination and appear floating about in the urine as shreds.

The small gramules are probably formed in the glands of the anterior urethra, and the larger plugs, shaped like a comma or tad- 
pole (Fuerbringer's hoolis), originate in the follicles of the prostatic urethra.

Larger "clap-shreds" are also always present, and are occasioned by the secretion from erosions drying upon the surface, forming a scab, which is washed away by the stream of urine.

Changes in the Mucosa.-During the acute inflammatory stage of a gonorrhoa the cylindrical epithelium lining the urethra is loosened and thrown off in patches, leaving superficial erosions. These losses of epithelium, except in rery rare instances, are not deep enough to deserve the name of ulcers.

On account of the round-celled infiltration of the submucous tissues the erosions do not heal readily, but remain uncovered by epithelium for a long time.

The blood-rossels in the submucons tissues send up newlyformed capillary loops, which traverse the infiltration in an upward direction, and as they grow toward the surface penetrate the floor of the erosion and convert it into a bed of newly-formed granulations.

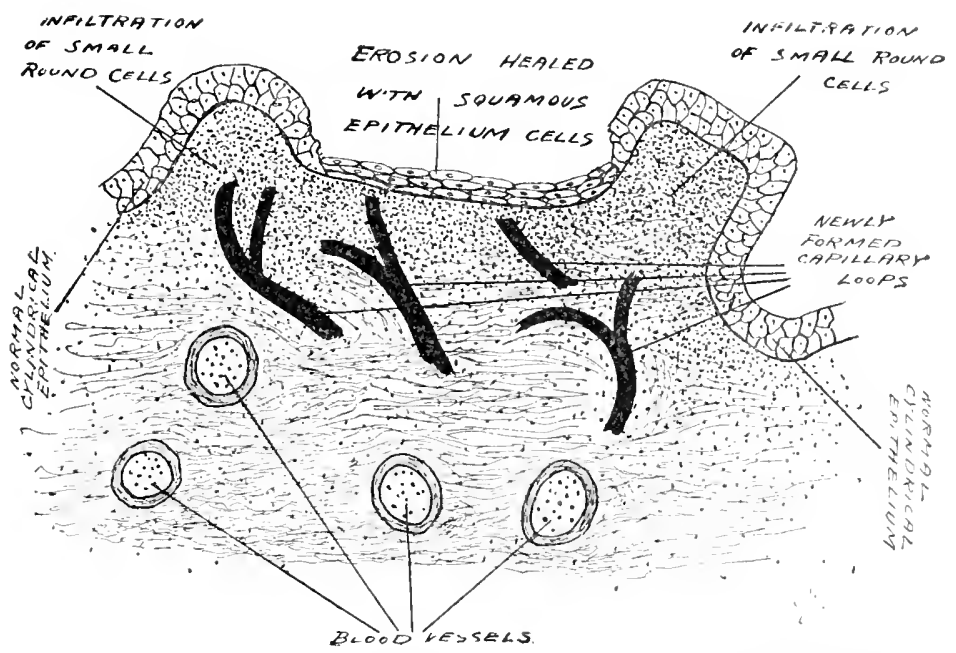

Fig. 10.-Diagram of a Section of the Urethra, Representing the Histological Changes in the Formation of a Granular Patch in Chronic Urethritis.

These "granular patches" resemble an uleer in any part of the body after it has become covered with luxuriant florid granulations, 
which are compoed purely of eapillary loops, having no tendeney to eicatrize and which ale easily broken down and destroyed by slight force.

In other case- the mucous membrane is not eroded and there are no gramular patches present. Instead of a loss of substance there is simply swelling. congetion, and urlema of the mucous membrane, in scattered patches. neasioned by its being in a condition of chronic intlammation and intiltrated with lencocytes.

These superficial changes in the mucous membrane octasion a continuous glrety discharge until they are healed.

Final healing of the lesions is brought about as follows:-

When the small romblecelled infiltration is tirst deposited, it is soft and sucenlent. and while in this state it may disappear, entirely or in part, by a process of absonplim.

If alssorption does not take place, the small, romnd, infiltrating eclls become organized, and are replaced by true fibrous connective tissue of a low grade, which goes on to contraction.

In a case where the infiltration was of the superficial, or mucous, form, involving only the mucons membrane and surrounding the glinds, stricture does not follow.

In the deep form of infiltration. however, which extends deeply into the periurethal tissues and involves the meshes of the corpus spongiosum extensirely, the heary masses of scar-tissue, into which the infiltration beomes converted. contract, imprair the dilatability or may materially decrease the calibre of the urethral canal, and form stricture.

After the intiltration which surrounded Morganits erypts and littrés gramis has been converted into scar-tissue, its subsequent eontraction squeceses the walls together; so that the glands are compressed and obliterated.

The granulations. which have formed upon the erosions, consist sinuply of capillary blood-ressels. which have been given off from the sulmucous resials anil have penetrated the infiltration in an upward direction. Dfter the formation of scar-tissue its contraction squeezes the capplliaries together and destroys them, and the gramulations disalperar as a result of stramgulation.

The erosions become eovered, not with the normal eylindrical "pithelimm of the halthy portions of the mucous menturane, but by mamy layers of squamous epitheliom.

The changes wonght he the conversion and contraction of the 


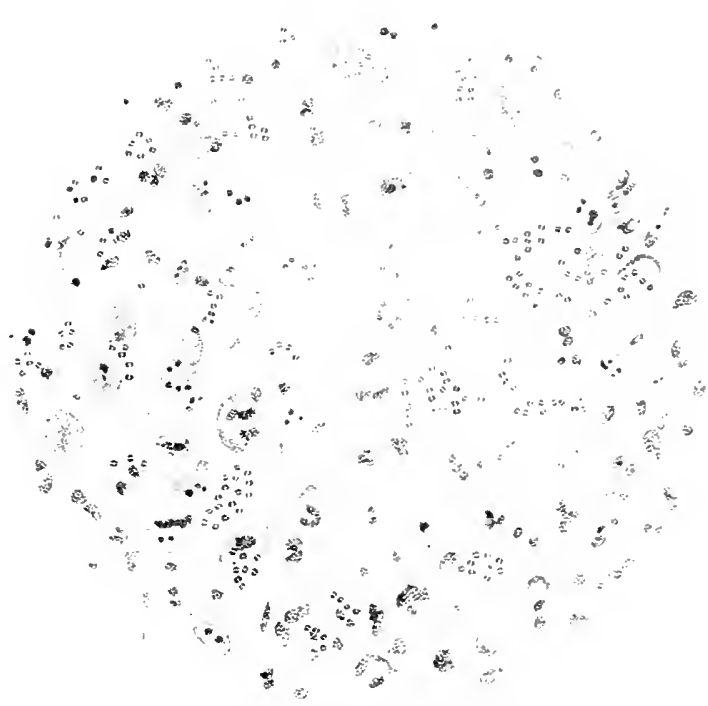

III. Conorrheal Rheumatism.

Synovial membrane infiltrated with numerous intracellular gonococel.

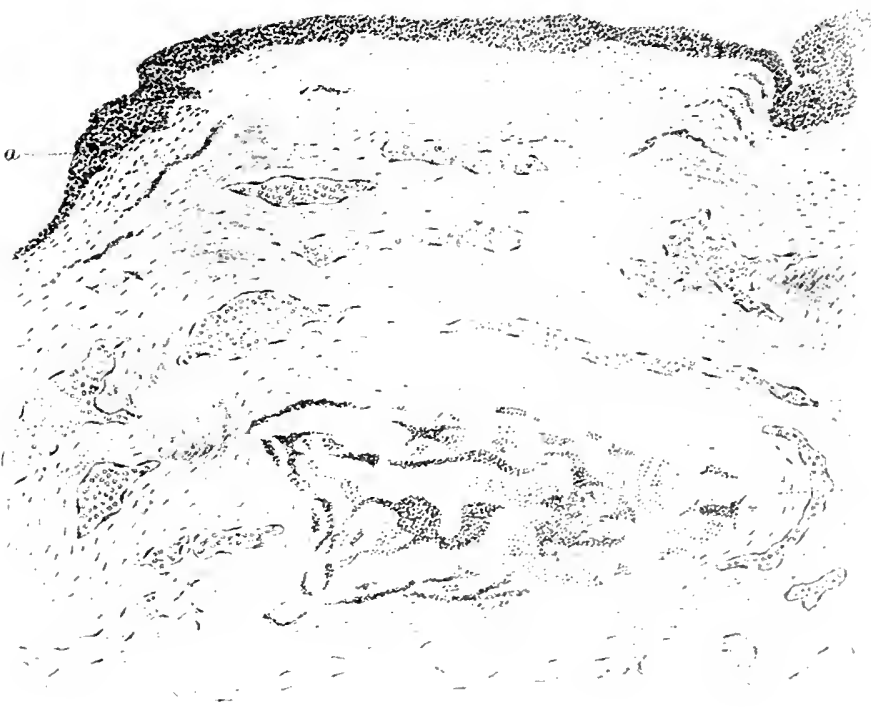

IV. Beginning Stricture.

$\boldsymbol{a}$, Squamous epithelium in many laỵers.

$b$, Contracted connective tissue.

$c$, Contracted meshes of the corpus spongiosum.

$d$, Remains of Littré's gland, obliterated through contraction of the periglandular and interstitial connective tissue. 

scar-tissue require from two to ten years for their completion, and they do not proceed with a uniform degree of rapidity: so that, on examining a case, all gradations of the process may often be seen at the same time.

\section{SYMPTOMS.}

There is an absence of any marked subjective symptoms: there may be at most an occasional tickling at the meatus.

The discharge from the urethra is muco-purulent, thin, and scanty, and is often so slight that there is only a drop in the morning or a sticking together of the lips of the meatus.

A common feature of chronic nrethritis is the exacerbalions which are constantly occurring. The patient derelops a profuse purulent discharge, which is checked very promptly by treatment.

As a result of various indiscretions, an acute inflammation is set up in the damaged portions of the urethra, and the discharge which is produced in them occasions a reinfection of healthy portions of the camal, as it passes over them.

When a considerable extent of surface of the mucous membrane is involved in the inflammatory process, if the patient passes his water into two glasses, the first glass is turbid from the quantity of pus washed out from the canal. On microscopic examination the discharge is found to be composed of pus-cells, containing gonocoeci in profusion, desquamated epithelial cells, and mucus from the crypts and follicles. After the inflammation is localized. and exists only in spots, the urine in the first glass is no longer turbid, but shows a few shreds floating in clear urine.

The presence of shreds always indicates that at some point along the urethra the mucous membrane is diseased, and a shred is simply the secretion, which forms a scab on the surface, and is washed off by the stream of urine. The form of lesion may be an erosion or granular patch or a chronic catinth of the mucous membrane lying over an infiltration.

Microscopic examination shows the composition of shreds to be pus-cells, which may or may not contain gonococei, and desquamated epithelium, held together by a quantity of muens.

In shape shreds present themscres as heary flakes; long, slender filaments; tadpole-shaped bodies, or small gramules. The heary shreds always contain pus, and sink to the bottom, while the light filaments are composed entirely of sipumous epithelial cells and float. The point of practical clinical importance to determine is whether the 


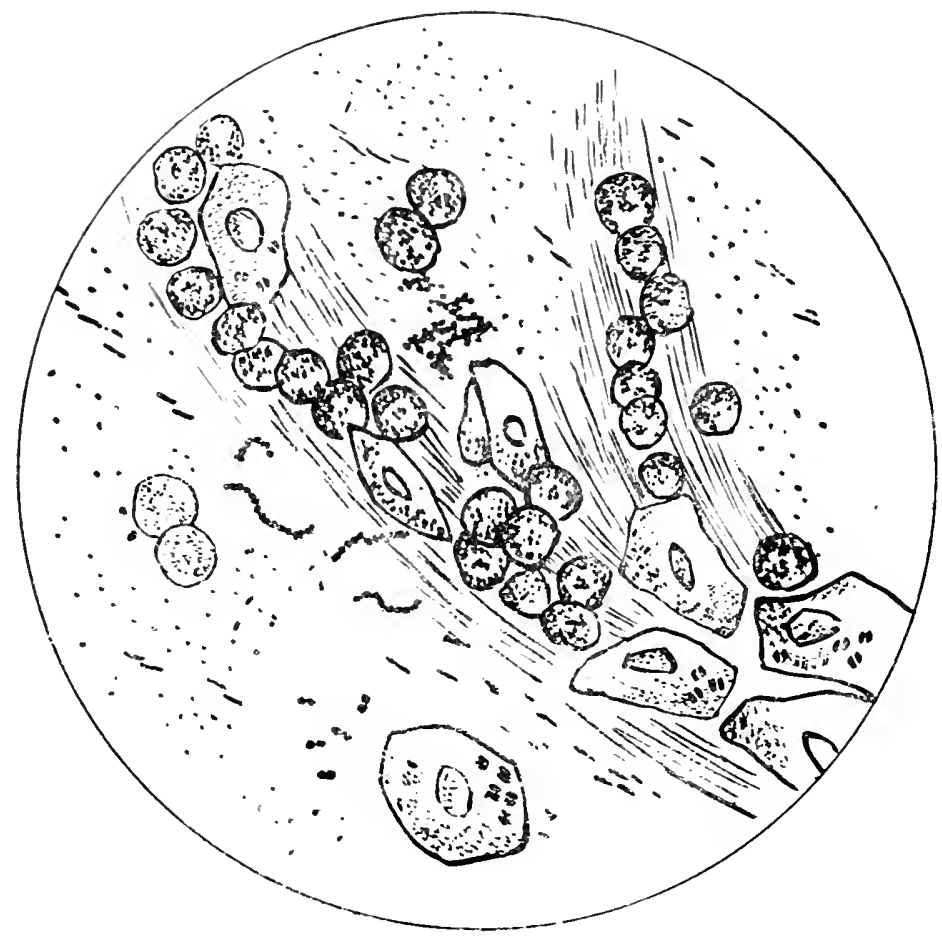

Fig. 11.- Sherl from a case of Gomorthral of long standing. Mixed Infection has oecurred. The sperimen shows dexplumated Squamons Epithelial cells, with comocueci on their surfaces, Puscells,

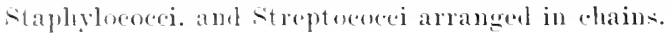

shred are made up of pus-eells containing gonococei, or whether they are composad of syumous epithelium alone, despuamated from the healed surface of a tormer lesion.

\section{DIAGNOSIS}

The peints to detrluine in making the diagnosis of the conditions in rhromic antrinos methritis are:--

(a) Whether a conciturable surfare of the urethral mucous membrane is involved in the inflammatory process, and secreting pus frecly. When this in tho ases it is indicated by a turbid. cloudy aplearaner of the tist glass of urine, on making the two-ghass test. $\mathrm{Or}_{\mathrm{r}}$ 
(b) Whether the inflammation is no longer general, but limited to localized areas. In the latter condition the first glase of urine will contain shreds floating in clear urine.

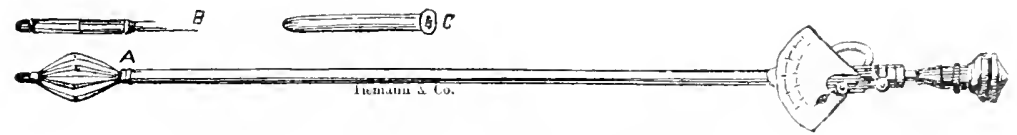

Fig. 12.-Oti, Erethrometer.

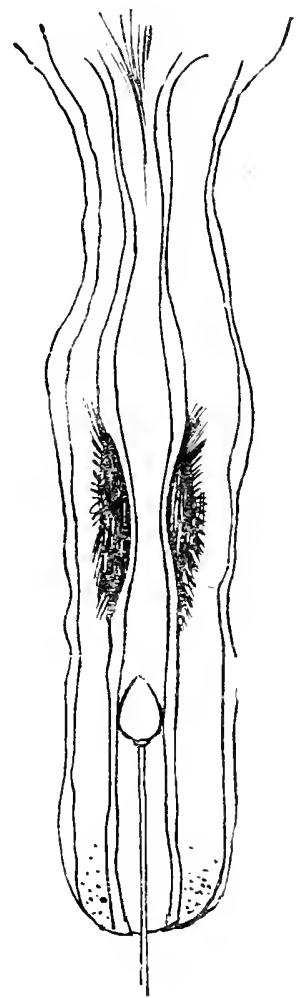

Fig l:s.

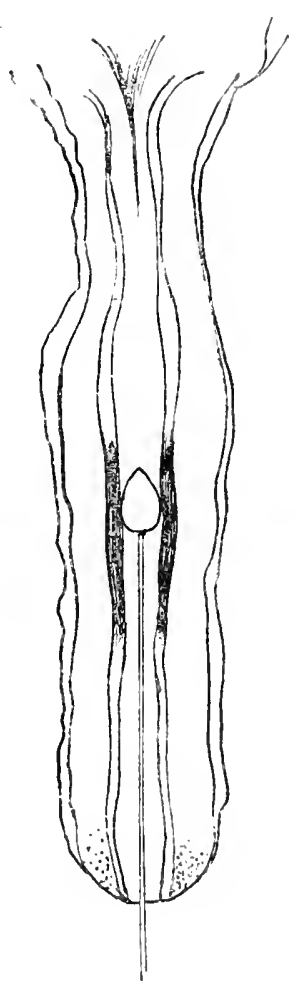

Fin. II

Fig. 13.-Diagram silowing method of detecting leep lufiltration, in Chronic Urethritis, with Bougie ì Boule or Urethrometer.

Fig. 14.--Diagram showing impossibility of recognizing Superforial Infiltration, inrolving Mucous Menbrane alone, by means of bougie à Boule or Urethrometer. 
It is equally important to ascertain if:-

(c) The inflammatory process is superficial; that is, limited to the mucous membrane and glands, or if:-

(d) The infiltration has involved the meshes of the corpus spongiosum, and commencing stricture is present. The instruments useful in settling points $c$ and $d$ are: (1) the Otis urethrometer, (2) the bulbous bougie, and (3) the endoscope.

Otis Urethrometer.-Method of Using.-The point of greatest dilatabilit!y of the normal urethra is at the bulb, and on withdrawing the urethrometer we find that the dilatability of the urethra is diminished gradually toward the meatus, except at the fossa navicularis. When a deep infiltration is present the dilatability of the methra is diminished abruptly, but the urethral canal is freely dilatable both before and behind the infiltrated point.

(on the other hand, superficial infiltrations involving only the mucons membrane do not extend into the deeper submucous tissues or mohes of corpus spongiosum and do not interfere with the dilatability of the urethral canal.

It is important to recognize deep infiltrations, while they are still soft and recent and before they have been conrerted with scartissuc, so as to bring about their absorption and prevent the formation of stricture.

The bulbous bougie, preferably the flexible rariety, may be used for the same purpose, but is better adapted to recognizing infiltratinns which have been transformed into star-tissue and begun to contract.

\section{TREATMENT.}

In the cases of chronic urethritis of the superficial variety- that is. when the diseased condition is limited to the mucous membrane, and does not affect the deeper tissues-the indication for treatment is to bring the inflammatory process to an end and promote the formation of squanous epithelium to cover the erosions. These indications are fulfilled by the local application of astringent and antiseltic solutions. Then general catarrh of the mncous membrane is present, as indicated by turbidity of the first glass of urine, the patient may inject his urethra with an ordinary gonorhoa syringe, and gradually increase the strength of the injections.

(For formulæ 
sce "Gonorrhoe.") It is preferable, however, to use an irrigator, which has the adrantage of distending the folds of mucous membrane and insuring a thorough contact of the solution with its cntire surface.

A soft-rubber catheter, attached to a large-sized hard-rubber syringe holding 4 ounces, carried down into the bulbons urethra, may be used, but is not as effective as the irrigator.

The best solution to use is the nitrate of silver, beginning with 1 in 5000 and increasing the strength to 1 in 1000 .

Permanganate of potash takes the second place as a curative agent, and should be used in the strength of 1 in 6000 or 3000 at the beginning and gradually increased to 1 in 1500 .

In cascs where no micro-organisms are present and a simple astringent is called for, Ultzmann's solution may be used:-

R Zinci sulphatis,

Pulvis aluminis Acidi carbolici,

Glycerini .............................. an mxij.

Distilled water ......................... q. s. at f f $\overline{5}$ s.

Sig.: Use half an ounce to a pint of water, and increase to one oumee to a pint.

All these solutions should be warm, and the irrigations should be made, in general, every second day, although occasionally they may be of use every day.

Isolated Foci.-After the disease has become older and the inflammation of a considerable surface of the mucous membrane has subsided, it still lingers in spots in the canal. It is no longer general, but localized to particular areas, and on examining the urine the first glass, instead of being tmrbid from pus, is clear, but contains clap-shreds, or filaments, floating in it. Nany of the localized cases heal under irrigation, but in those cases which prove obstinate concentrated solutions must be applied directly to the localized diseased spots by means of ( $a$ ) instillations with a Guyon or Cltzmann syringe, (b) Ultzmann's brush apparatus, or $(c)$ the endoscope in rery exceptional cases.

Instillations.-By means of Guyon's or Ultzmann's syringe concentrated solutions of nitrate of silver can be deposited, drop by drop, along the whole length of the urethra, from the resical splinincter to the meatus, thus bringing the medicament in contact with healthy and diseased portions alike. 
It is rerrexeptional for the diseased foed to be so isolated that they can be trated in any other way, even through the endoscope.

The indication for the nse of instillations of nitrate of silver is considered to be the mesence of clup-shreds floating in clear urine.

In a casc coming under treatment, however, for the first time, instillations shonld never lie used until the effect of irrigations has been tried. for the treatment of chronic gonorrhea is founded upon the principle of beginning with mild applications and gradually proceeding to the use of stronger and more irritating measures.

Another point to bear in mind is the fact that the irritation cansed ly the application of concentrated and canstic solutions may in itself prolong the inflammation and prevent the recovery. So that it is always desirable, in a case which has been energetically treated, to interrupt, for two or three weeks, all local applications, in order to allow the irritation cansed by them to subside.

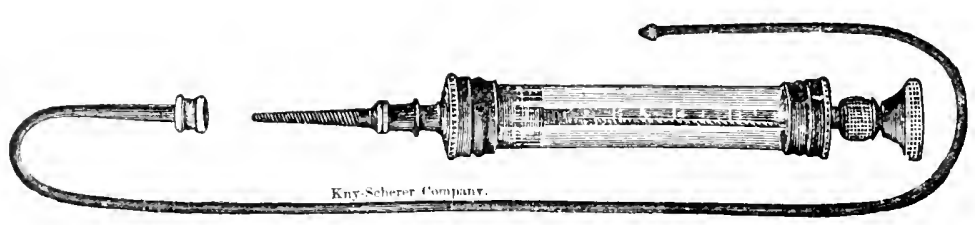

Fig. 15.-Guyon's syringe.

In bewinning the treatment with instillations, 15 drops of a 2grain-to-the-ounce solution of nitrate of silver shonld be nsed, which cances sone reaction. Jurning on urination and increased secretion, which lasts for twenty-four hours and then subsides.

In crery case where the presence of posterior urethritis is susJectent. the point of the syinge should be carried beyond the ent-off mucle and a few drops of the solution deposited along the posterior urethra: and as the syinge is withdrawn the anterior urethra is "otched." a drop at a time. along its entire length.

Ther instillations should be male every two dars and used two, three, or fonr tines, or, in fact. as long as a diminution in the shreds is notur. By this time the methra has become acenstomed to the irritation of the solution, and its strength must be increased to 5 grains to the ounce. 'l'he strength of the solutions are gradually increased in this way until the patient is well or until a strengtl of 25 grains to the onnce is attained. 
The patient may be allowed to use a mild astringent injection, upon the days when no instillation is made.

During the comrse of the treatment exacerbalioms, accomprnied by resical tenesmus, free suppuration, and turloidity of both wlasses of urine, occur at times. When these happen, the instillations must. of course, be suspended and either sandal-rood oil or salicylate of soda given by the month, or, if the exacerbation is not rery serere, the ease may be treated by irrigations until the mine is again free from pus, when the instillations may be resumed.

Cases of chronic anterior methritis which are several years old, and where the pathological changes of erosion and granulation formation are very extensive and the gonococci have entirely disappeared from the tissues, sometimes resist the nitrate-of-silver applications. In such cases linger recommends instillations of sulphate of copper, beginning with 25 grains to the ounce and increasing to 50 , 55 , and 100 grains. This is only to be used, howerer, after the nitrate of silver' is found to be ineffective.

'The first instillation of sulphate of copper is said either to cause a diminution in the number of shreds or else, in the erent of gonococei still being in the tissues, to occasion a profuse purnlent discharge. Hence it is that the presence of gonococci is considered a contraindication to the sulphate-of-copper treatment, and, in the erent of gonococci being still present, a reversion to the nitrate of silver is again in order.

Ultzmann's Brush Apparatus.-A few years ago a favorite method of treating chronic anterior methritis was by means of the brush apparatus, but of late years the treatment by irrigations and instillations has largely supplanted it.

It is usually the case that when the bully is affected the remainder of the mucous membrane is by no means healthy. If the entire anterior urethra is brushed over, by a combined rotary and withdrawing motion of the brush, with a solution of nitrate of silver from 15 to 25 grains to the onnce in strength and examined immediately afterward with the endoscope, the diseased spots will be seen to be colored a whitish gray. while the healthy portions of mucous membrane will appear maltered by the nitrate of silver. In this way the growth of epithelium is stimulated over the croded spots. The anterior urethra should be brushed orer every second day or even every day. In obstinate cases of long standing sulphate-of-coppor solutions may be used in the same way. 
Treatment with the endoscope is only applicable to a few cases, viz.: those in which the discase process is exceedingly circumscribed, and these are exceptional in point of frequency. Examination with the endoscope determines that the urethra is healthy in the main, but that certain isolated spots are diseased. If the crypts and follicles are affected, their walls are elerated and of a dark-red color (glandular and periglandular urethritis), or there may be one or two patches of granulation in the urethra. In either case we can apply a strongly concentrated solution directly upon the diseased spots, through an endoscope, by means of a cotton swab, without its coming in contact with the healthy mucous membrane.

Nitrate of silver in solution as high as 20 per cent. or copper sulphate up to 10 per cent. can be used. Destruction of the diseased chands with a galvano-caustic is necessary only in cases of extreme rarity.

In general, the value of endoscopic treatment is limited, as there are very few ases wlicre the pathological changes are confined to one or two isolated spots, and in others the cause of the continuance of the persistent secretion are changes underneath the surface of the mucous membrane, which are not recognizable by inspection.

The deep form of chronic anterior wrethritis in which, in addition to disease of the mucous membrane, an infiltration composed of small round cells is present in the submucous tissucs, even cxtending into the meshes of the corpus spongiosum, must be treated on different lines from the superficial variety of urethritis. In these cases the infiltration lying underneath the mucous membrane cannot be reached by applying astringent or bactericidal solutions to its surface, and, while the secretion may be held temporarily in check, an exacerluation occurs on the slightest provocation.

The indications for treatment are:-

I. To promote the absorption of the infiltration and restore the easticity of the urethral wall.

II. To subdue the existing superficial inflammation in the mucous membrane and glands.

III. To destroy the gonococci, which are harbored in the substance of the infiltration and in the urethral glands.

The first indication-that is, the promotion of the absorption of the infiltration-is met ly the passage of a steel sound large enough to distend the urethra fully and put the ring of infiltration upon the stretch. 
The therapentic effects of the passage of sounds are:-

I. To allar urethral hyperasthesia. The passage of the sount blunts the extreme sensitiveness of the nerve-filaments and abolishes any spasmodic contractions of the museular fibres which may be present.

II. The infiltration lying beneath the mucous membrane renders it rigid and impairs its elasticity. A sound large enough to distend the urethra stretches the infiltration and canses small tears in its substance beneath the mucous membrane. A traumatic inflammation. with increased rascularization. results from these tears, and absorption is stimulated.

III. The passage of a sound expresses and squeezes out the contents of the suppurating urethral crypts and follicles.

IV. The stretching of the urethral walls by the sound brealis down granulations and stimulates the formation of epithelinm upon eroded spots.

In order to meet these indications it is necessary to use a somd

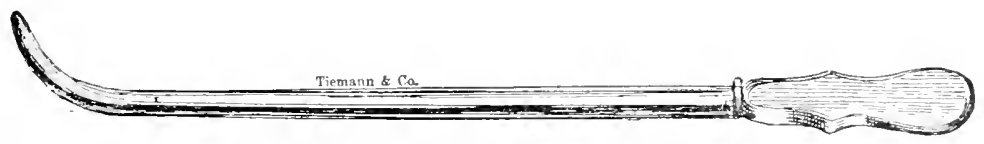

Fig. I6.-Steel Sound with Tan Buren Curve.

of large calibre, which wiil fully distend and stretch the urethral canal.

The sound should not be passed too frequentiy. In cases of soft and recent infiltration the intervals may be from two to four days, always waiting until the reaction following has subsided. In cares of hard, organized infiltration the interrals should be longer: from five to eight days.

If the meatus is too narrow to admit a sound of sufficient size, it should be divided upon the floor.

There are, however, certain cases where it is undesirable to enlarge the meatus, as, for instance. in hypospadias, and, again. there are other cases where the urethra is so capacions that a To. 30 French sound will lie in it loosely without stretching the walls or compresing the infiltration. In these cases recourse may be had to the Oberlaender dilator.

The action of the dilator is to tear apart the infiltration under- 
neath the mucous menturme, which remains intact without being woumded. 'This is an important feature of dilatation, since any fresh womut of the mucoms membrane would open up a passage for renewed infection with micro-organisms.

() Herlacuder claims that a fresh inflammation starts up from the tears in the infiltration, lut the increased vascularization occasions its alisorption.

'l'he dibatation should be rery gradual,-one or two numbers at a sitting,--and slowld not be performed oftener than once a week, and care should be taken not to lacerate the mucous membrane. If this accident llould occur, it will be announced by bamorrhage from the meatus.

It makes no diflerence, as far as treatment is concerned, whether the sulumucoid round-celled infiltration is soft and recent or whether it has hen transfomed into scar-tissne. The indications in either Gace ale. ly dilatation and pressure, to promote its absorption. Cases which are quite recent-that is, less than from two to six months oldare make worse by attempts at dilatation. Cases in which a eonsiderable surface of mucous membrane is involved are msuitable for dilatation, until the catarn has been checked by irrigations and the superficial proces is localized to a few spots in the urethra. as denoted by shreds flonting in clear urine.

The second and third indications-which are to cure the inflammation in the mucous membrane and glands and destroy the gonococci-have been ahready considered under the treatment of the superficial form of urethritis, and the measmres adapted to these ends should be combined with the dilatation.

It is always desirable to use irrigations after dilating with a sound or dilator. The folds of the mucous membrane are smoothed out by the pressue of the solum and the solution comes in contact with the whole -urface. If small tears have occured in the mucous membrane from strotching. the irrigating fluid seals them up and prevents infertions.

Instillations uf concentrated solutions are not admissible upon the smon thy of the dilatation, but should only be used two or three dali- later. 


\section{CHRONIC POSTERIOR URETHRITIS.}

The posterior urethra is involved in about 80 per cent. of all cases of acute gonorrhca. In many of these the disease never becomes chronic, but, when it does, the posterior urethra remains inflamed quite as often as the anterior.

Chronic posterior urethritis may exist alone, the inflammation having run its course and ended in the anterior part of the canal, but we frequently find a chronic inflammation of both anterior and posterior portions of the urethra, at the same time.

Acute posterior urethritis is almost invariably caused by gonorrhœa, but a chronic inflammation can be occasioned in other ways. Any cause which tends to produce a prolonged state of congestion in the posterior urethra which is oft repeated will, in time, lead to the establishment of a condition of inflammation in the mucous membrane and hyperplasia of the submucous tissues.

The causes which are usually responsilule for these conditions are excessive sexual intercourse or sexual abuses, such-as masturbation or coitus reservatus (withdrawal).

For all practical purposes, the symptoms and treatment of chronic posterior urethritis may be considered together, without regard to its etiology.

\section{PATHOLOGY.}

On account of the abundant supply of glands and follicles and the thickness and vascularity of the mucous membrane the pus-formation is apt to linger in the posterior urethra for years, and is very apt to attack the prostate and scminal vesicles.

The histological changes are substantially the same as in chronic anterior urethritis.

The mucous membrane is in a state of chronic inflammation, with desquamation of its epithelium, and the submucous tissues are the scat of a small round-celled infiltration, which also surrounds the mucous crypts. The infiltration, in time, becomes converted into scartissue and the glands are obliterated by its pressure. The infiltration, however, is not transformed into distinct bands of scar-tissue, such as form strictures in the anterior urethra, but there is simply a general condensation or fibrous hardening of the periurethral tissues.

The sclerosis of the submucous tissue does not materially narrow the calibre of the urethra, and, consequently, stricture never occurs in the posterior urethra, except from traumatic origin. 
The verumontanum, or colliculus seminalis, is always affected in chronic posterior urethritis. It is enlarged, the mucous membrane is bluish in color and softened, and the natural sensitiveness is aggrarated to a high degree. This structure is the point most highly supplied with nervous filaments in the urethra, and, on account of its increased receptive influence to painful impressions, when the submucous infiltration begins to contract it compresses these nerves and occasions marked reflex disturbances.

The symptoms are often mental, and take the form of hypochondria, depression, irritability, and inability for sustained mental effort, or may be neuralgic in character and referred to distant or associated organs.

\section{DIAGNOSIS.}

The two-glass urine test is only applicable to cases where there is a considerable amount of pus-formation. In the following instances the posterior urethra may be chronically inflamed and the second glass of urine will not be discolored:-

(a) When such a small quantity of pus is secreted that it does not flow back and discolor the urine in the bladder.

(b) When the stream of urine is feeble in force and not sufficient to wash out the mucous plugs from the crypts and follicles. Contraction of the muscular structures surrounding the urethra is necessary to accomplish their emptying.

(c) The prostatic crypts and seminal vesicles may be chronically inflamed and yet the pus formed does not flow out freely enough to appear in the urine unless direct pressure is made upon those organs by means of the finger in the rectum.

The Jadassohn-Van Zeissl method is useful in overcoming objections $a$ and $b$. The technique is as follows:-

The anterior urethra is irrigated by means of a catheter attached to a syringe or irrigator, which is carried down to the cut-off muscle. After the anterior urethra has been thoroughly cleansed the patient urinates in a glass, and the urine contains the pus or shreds washed out from the posterior urethra. The patient then passes the remainder of his urine into another glass, which represents the condition of the urine which had accumulated in the bladder.

After both anterior and posterior urethras have been cleansed of accumulated secretions by washing out and urinating, the secretions from the prostatic follicles and seminal vesicles should be collected by 
means of Jadassohn's expression urime test. By means of a finger in the rectum a pressure or massage is exerted upon the prostate glant, and the seminal resicles and their contents are squeezed out into the urethra. The patient then urinates and washes out the expressed secretions into a glass. The mine in the glass containing the secretions expressed from the prostate and resicles is called the expression urine.

In examining cases of chronic posterior urethritis we should always pay particular attention to the condition of the seminal resicles and prostatic follicles. These organs are very liable to be atfected by an extension of the gonorrhoal inflammation from the urethra, and when once attacked the gonococei are very likely to remain in them and continue to propagate for months and, indeed in some cases, for years.

\section{SYMPTOMS}

If the posterior urethra alone is diseased, there is an absence of purulent discharge from the meatus.

In the later stages of the disease there is but very slight pusformation, simply a congestion of the mucous membrane, with an infiltration and condensation of the submueous tissues. But in the early stages and in exacerbations, the pus-formation may be in considerable quantity, and will be easily shown by making the two-glass urine test.

On account of the chronic inflammation, the posterior urethra is always in a state of exaggerated sensitiveness, and the necessity for frequent urination is nearly always present. Usually the desire to urinate is so urgent that the patient cannot wait, but must respond at once to the call, or the urine escapes and wets his clothing.

Sexual symptoms are nearly always prominent. The sexual appetite is disturbed. There is either no inclination for coitus and, if indulged in, a condition of general nerrous depression follows, or else there may be a constant desire for sexual intercourse, which is not satisfied by indulgence.

The act of coitus is not satisfactorily performed. Ejaculation occurs prematurely on account of the irritable condition of the posterior urethra, and for the same reason seminal emissions, or pollutions, occur at night during sleep, nore frequently than is natural, and in some cases the seminal discharges may be stained with blood, which is derived either from the congested posterior urethra or else from an inflamed condition of the interior of the seminal vesicles. 
Mental symptoms are a striking feature of most cases of posterior urethritis. The patients are hypochondriacal, they suffer from depression of mind, and are low-spirited, melancholy, and despondent, and in extreme cases may have suicidal impulses. In this frame of mind they fall an easy prey to quacks and charlatans, whose advertisements guaranteeing to "restore lost manhood and relieve the evils attendant upon the errors of youth," appear in the columns of the daily papers.

\section{TREATMENT.}

In chronic inflammation of the posterior wrethra resulting from sexual excesses or abuses, the anterior urethra is not involved; but in posterior urethritis caused by gonorrhœa the anterior urethra is generally affected as well.

For purposes of treatment it is well to divide the cases of posterior urethritis into two groups:-

(a) Superficial, in which the mucous membrane and glands alone are involved in the inflammatory process.

(b) Deep form, in which, in addition to the inflammation in the mucous membrane, there is an infltration in the deeper tissues.

The small round-celled infiltration is deposited here and ultimately is converted into scar-tissue. It does not contract and form fibrous bands, but mercly produces a general condensation and hardening of the submucous tissue.

In the superficial form, where there is a considerable amount of pus-formation, irrigation by means of an irrigator or syringe and soft-rubber catheter introduced beyond the cut-off muscle, using nitrate-of-silver or permanganate-of-potash solution, will generally check the secretion. After the suppuration has lessened instillations with Lltimann's syringe, carried behind the cut-off muscle into the posterior urethra, of 15 drops of a nitrate-of-silver solution, beginning with 2 grains to the ounce and increasing as directed in the treatment of "Chronic Anterior Urethritis," usually causes a prompt disappearance of the remaining secretion and shreds. If it is desirable to medicate the posterior urethra without having the nitrate-of-silver solution come in contact with the interior of the bladder, it is well to make the instillation with the bladder full of urine. In that event any of the solution which flows back into the bladder is neutralized by the salts of the urine.

If, on the other hand, we wish to affect the base of the bladder 
as well as the posterior urethra, the bladder should be emptied of urine before making an instillation.

In treating the deep form of chronic posterior wrethritis the indications are:-

I. To cure the inflammation in the mucous membrane.

II. To produce absorption of the deep-lying infiltration.

To accomplish these objects it is necessary to employ, in adrition to the irrigation or instillation, the regular passage of sounds. Forcible dilatation or tearing apart of the tissues is harmful, and we should proceed with the utmost gentleness and caution in the manipulation of sounds in this region. The sound, of course, should never be passed until the free suppuration has ceased and there are only very chronic and indolent inflammatory residua remaining, on account of the danger of increasing the inflammation or of causing epididymitis.

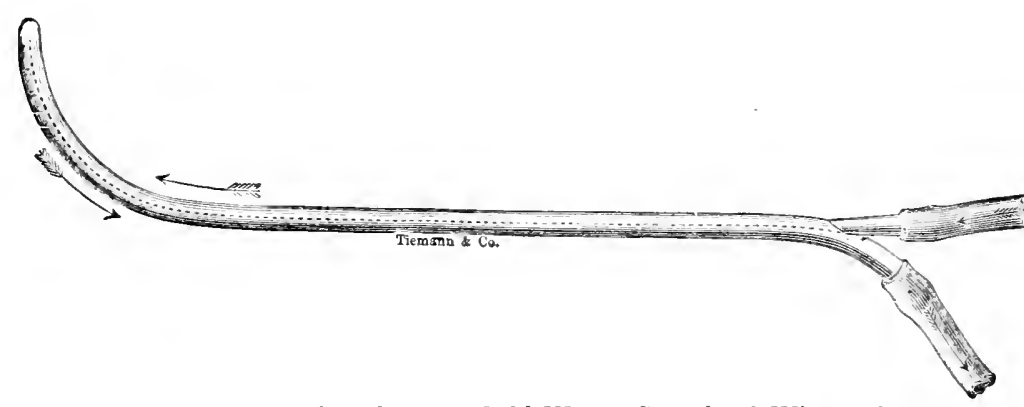

Fig. 17.-Psychrophor, or Coll-Water Somml, of Winternitz.

The Benique sound is particularly adapted to the posterior urethra, on account of its shape, which is similar to that which a soft catheter assumes when it lies in the bladder and urethra. The weight of the Benique sound has some advantage, as it produces a certain amount of compression and so stimulates absorption of the infiltration, besides emptying out the crypts and follicles.

The cases of chronic posterior urethritis which require the nse of Kollmann's posterior dilator are exceptional in frequency. When the dilator is used in the posterior methra great care should be exercised not to dilate too rapidly and so lacerate the tissues or excite epididymitis, as the tolerance to instrumentation is far less in the posterior urethra than in the anterior.

The psychrophor, or cold-water sound, made preferably with the 
Benique curve, is found to be of more benefit than the ordinary steel sound, in the cases which develop a chronic sexual neurasthenia or have local symptoms pointing to excessive irritability of the posterior urethra, such as unduly frequent seminal emissions.

The psychrophor should be used erery sccond day for ten or twenty minutes. The effect of the cold is to diminish the hypersensitireness of the rerumontanum, and the pressure of the sound acts beneficially in promoting absorption.

In every case of chronic posterior urethritis the condition of the seminal resicles should be investigated by rectal examination. It is uscless to attempt to cure an inflamed posterior urethra when a pair of inflaned seminal vesicles are discharging a quantity of gonorrhoal pus into the urethra every few days and cansing an exaccrbation. Many cases of relapsing posterior mrethritis will get permanently well through a systematic stripping of the seminal resicles, when everything else has been tried in vain.

At the same time the prostate should not be overlooked, for a frillicular prostatitis is often present as a complication, and should be traited by massage through the rectum, in order to empty out the contents of the inflamed and dilated prostatic crypts.

\section{SUMMARY OF TREATMENT OF CHRONIC ANTERIOR AND POSTERIOR URETHRITIS.}

In the great majority of cases of chronic urethritis a systematic and regular conrse of dilalations. with sounds or Oberlaender's dilator accompanied by copions irigations of wcak astringent solutions, will be more succesful then any other plan of treatment. Before beginning dilatation the inflammation of the mucous membrane should no longer be general. but confined to localized areas.

When the inflammation is general, the urine passed in a glass is turbid and cloudy from admixture with pus, but the localization of the inflammation to isolated spots is indicated by shreds floating in clear urine.

After dilatation and irrigations have been carried out for some time and the patient is not cured, as shown by persistence of morning drop and shects. concentrated solution of nitrate of silver may be applied directly to the inflamed areas by means of:-

(a) T'ltzmann's bu'ush apparatus.

(b) Guyon's or litzmann's syringe. 
(c) The endoscope.

In every case of chronic posterior urethritis the condition of the seminal vesicles and, prostate should be ascertained, and, if diseased, they should be treated by stripping or massage through the rectum.

\section{PROGNOSIS OF CHRONIC ANTERIOR AND POSTERIOR URETHRITIS.}

The duration of the disease is always protracted, and requires great patience on the part of physician and patient.

The test as to when the patient is cured is the permanent absence of pus-cells and gonococci in the urethral secretions.

When all discharge has ceased from the anterior urethra, in order to get material for microscopic examination it is necessary to inject a syringeful of 2 -per-cent. nitrate-of-silver solution into the anterior urethra. A free discharge of pus follows, which ceases in twenty-four hours. If no gonococci are found in the resulting secretion after microscopic examination of several smears, it is safe to conclude that the micro-organisms have all been eliminated from the urethra in front of the cut-off muscle.

The secretions of the prostatie crypts and seminal vesicles should be expressed by the finger in the rectum and examined at the same time. It is from these organs that the chief danger of infection comes after discharge has ceased from the meatus, and they are responsible for the majority of cases of infection of wives and mistresses. In examining the expressed secretions from the prostate and vesicles it is not enough to kemand absence of gonococci; we should insist upon an absence of pus-cells, for if pus-cells are present there may be an occasionál gonococcus in a cell which might easily be overlooked.

It is estimated that one-eighth of all the diseases of women are occasioned by gonorrheal infection attacking the vagina or cavity of the uterus, and thence extending to the Fallopian tubes and ovaries. In view of these facts, a patient who has suffered from chronic gonorrhoa should only be allowed to marry when examination shows:-

I. Absence of gonococci.

II. Absence of pus-cells.

III. Freedom from strieture.

IV. A healthy normal condition of prostate and seminal resicles.

Certain cases of chronic urethritis suffer from excess of treatment, and the inflammation is maintained by the irritation produced by the local applications. 
After a case has been under treatment for a couple of months, it is always desirable to stop all injections or instillations for ten days in order to make sure that the natural process of recovery is not retarded by ovcrtreatment.

Entirc disappearance of shreds is not to be looked for. The erosions have becn covered with many layers of squamous epithelimm, and continuance of desquamation of the upper layers goes on as the new cells are formed in the depths.

'Tlie shreds appear as thin filaments which float in the urine, and microscopic examination shows them to be composed of squamous cells alone without containing any pus-corpuscles.

\section{METHOD OF EXAMINING A CASE OF CHRONIC URETHRITIS.} First Day.

History: Take in detail.

I. Inspect pus squeezed from meatns.

II. Examine urethra with bulbous bougie or urethrometer.

\section{Second Day.}

III. Wash out shreds and seeretion from anterior urethra.

IV. Patient urinates in glass No. 1. No. 1 contains washings from posterior urethra.

V. Examine prostate and seminal vesicles per rectum.

VI. Patient urinates in glass No. 2. No. 2 contains expression urine from prostate and seminal resicles. ally.

VII. Examine shreds and pus secreted by urethra microscopic-

\section{Third Day.}

VIII. Examine urethra with endoscope unless a considerable portion of the mucous membrane is inflamed and secreting pus freely.

\section{URETHROSCOPY.}

The use of the urethroscope or endoscope is disappointing in many cases, inasmuch as the diseased processes in the urethra occur chiefly unterneath the mucous membrane, and the surface is often but little afiected. 
It has a field of great usefulness, however, for disclosing and treating diseased processes which are strictly localized to one or two isolated foci, and it is useful in diagnosis and also enables us to olserve the effects of treatment by dilatation and irrigation.

There are many varieties of urethroscopes in use, and after using most of them I have come to prefer the simple Klotz tube, illuminated by means of a light reflected with a head-mirror from a powerful central-draft oil-lamp. (The Belgian, Mitrailleuse, or Rochester lamps are suitable.)

The beginner in endoscopy should select the shortest and widest tube which will pass the meatus, doing a preliminary meatotomy if necessary. It is very difficult for even an experienced urethroscopist to see through a tube which is less than No. 24 French in calibre, and as larger tubes are used the examination becomes correspondingly easier. The tubes which are indispensable are Nos. 21,26 , and 30 French,

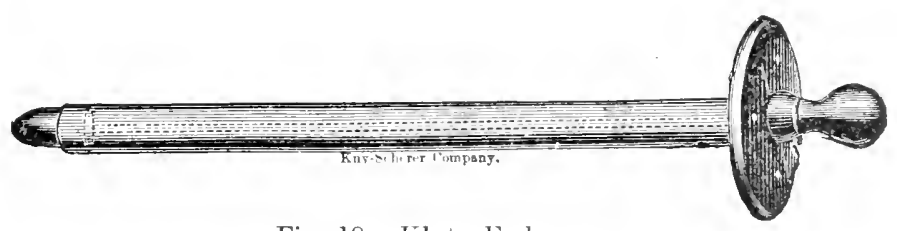

Fig. 18.-Klotz Endoscope.

each four inches long, and Nos. 26 and 28 French, each six inches long. With the short tubes the anterior urethra is examined, and the posterior urethra by means of the long ones.

Normal Appearances of Anterior Urethra.-I. Observe the surface of the funnel-shaped figure which the urethral walls assume beyond the end of the endoscope.

II. The central figure: i.e., appearance presented by the closed lumen of the urethra, which forms the apex of the funnel.

The folds of mucous membrane radiate from the central figure outward toward the periphery. Their thickness and number indicate if the mucous membrane be infiltrated, and are of diagnostic importance. Longitudinal red stripes running toward the central figure are also noted, and indicate a normal uninfiltrated condition of the mucous membrane.

The surface of the mucous membrane is smooth and glistening and with a distinct lustre; its color is ordinarily pale and rosy, but 
if normally hyperæmic may appear distinctly red or purple, without being diseased.

The openings of Morgarnirs crypts appear in the roof of the urethra as red speclis or small slits as large as a pin's head.

The posterior urethra prescnts substantially the same appearances as the anterior, except that in addition the rerumontanum is scen upon the floor of the urethra looking like a fold of mucous membrane.

In cxceptional cases the ejaculatory ducts can be seen as little dots alongside the rerumontanum.

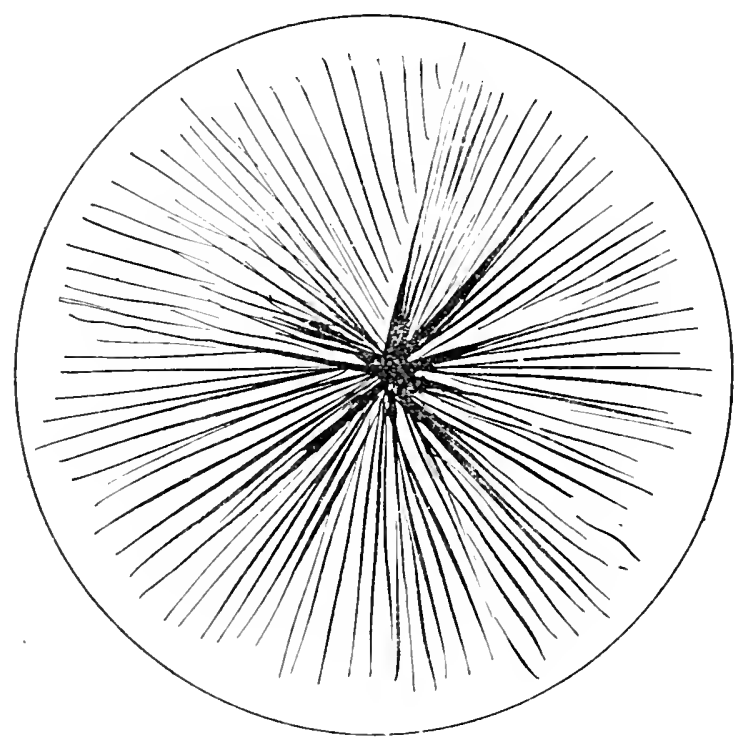

Fig. 19.- Urethroscopic picture of a Normal Urethra, showing a multitude of fine folds and small C'entral Figure.

Pathological Changes. - Chronic urethritis is divided into two forms:-

(a) Superficial: i.e., affecting mucous membrane and glands withont involving the deep tissues.

(b) Deep form in which, in addition to the involvement of mucous membrane and glands, a round-celled infiltration occurs in the deeper lying submucons tissues, which is ultimately converted into fibrillated connective tissue. 
The new fibrous tissue causes a rigidity of the urethra, and. as it contracts, produces a narrowing of the urethral calibre, or stricture, , and also destroys, by compression, the urethral glands which it surrounds.

On viewing a case of the superfirial form of chronic urethritis endoscopically, we note a swollen, cedematous, and puffy condition of the mucous membrane at the diseaser points. The folds, instead of being numerous and fine, are thick and broad.

Attention should be paid to the Tustre of the unucous membrane.

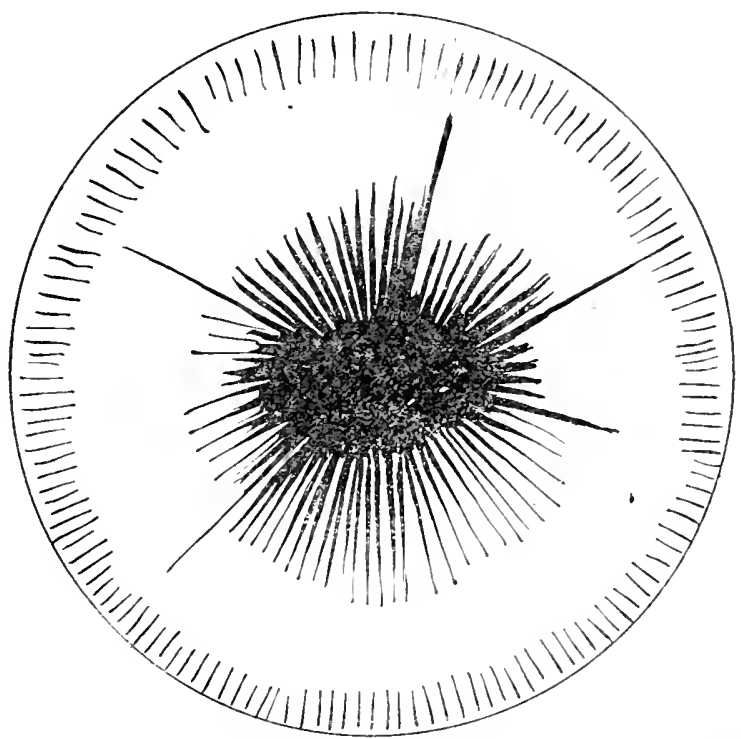

Fig. 20.-Urethroscopic picture of Soft Infiltration of the Mucous and Submucous Tissues. The Central Figure is Wide and Gaping, and the Folds of Thickened Mucous Membrane are Few in Number and Coarse and Broad.

The epithelial layer forms a smooth transparent covering with a distinct uniform lustre, which is increased when the mucous membrane is congested and swollen, and diminished when it is infiltrated or when the epithelium is desquamater.

Erosions occur in consequence of the desquamation of epithelium, which, when extensive, uncovers the orifices of Littrés glands. They beeome visible as small, round, red specks arranged in groups pro- 
jecting above the floor of the eroded surface. Later in the disease the erosions often become covered by a growth of eapillary loops from below, which converts them into beds of granulations. These appear. of the size of small grains of sand, or may only eause a papillated appearance upon the surface of the mucous membrane, and bleed freely upon the introduction of the endoscopic tube.

Granulations are generally associated with an infiltration of the deeper submueous tissues.

In rare cases silvery-white spots may be observed upon the sur-

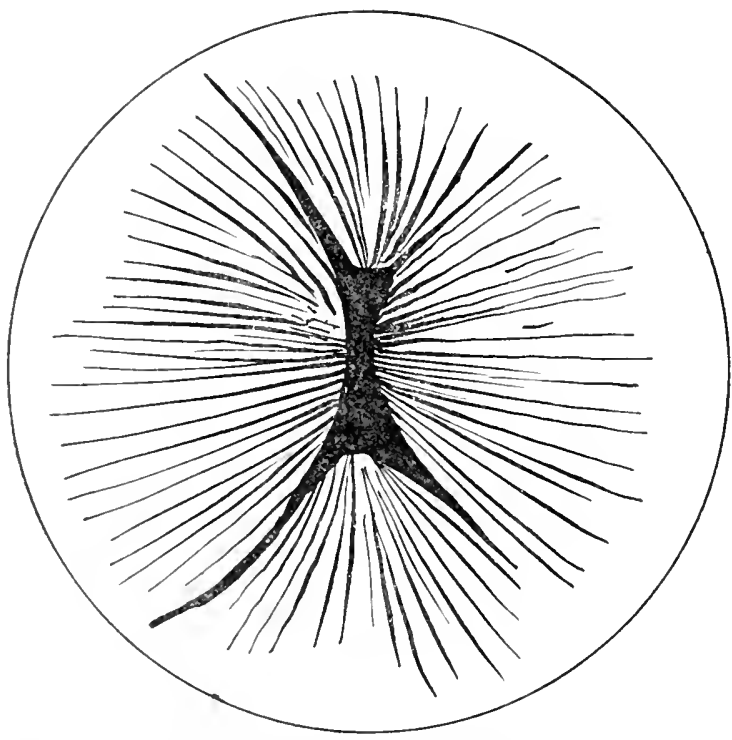

Fig. 21.-Urethroscopic picture of Hard Infiltration of the submucous tissnes. The central Figure is open, gaping, and very irregular in shape.

face of the mucous membrane, resembling the scales of psoriasis, and hence called by Oberlaender: "Psoriasis of the mucous membrane of the urethra."

Morgagni's crypts appear, not singly, but in groups, as small, red points, or depressions. Their openings are swollen and are patulous, and sometimes pus can be seen oozing from them. Later on in the disease, after periglandular infiltration has taken place, these openings gape, and are surrounded by a prominent rim of infiltration. 
Upon examining a case of the deep form of chronie urethritis in which the infiltration has become converted into fibrous tissue (hard infiltration-Oberlaender), we note that the central figure, instead of being romd, is irregular, and gaping widely. The folds of the mucous membrane have disappeared and the color is grayish.

Morgagni's crypts gape widely open, and are often seen surrounded by a ring of inflammation (periglandular infiltration). Littré's glands are sometimes visible, appearing as red speeks in a grayish, discolored mucous membrane.

In another class of cases affected with deep infiltration the openings of the glands are not apparent, as they are covered by the epithelium, which has a dry, dull appearance, and desquamates freely in spots.

According to Oberlaender, under treatment by gradual dilatation the restoration of the tissues to their normal condition can be watehed endoscopically, and after a chronic urethritis has been entirely healed the appearances are as follow:-

The epithelial layer has grown smooth and possesses its normal lustre and color. It has regained its natural condition of very minute and abundant folds. The infiltration disappears around the glands, although their mouths may still remain patulous and visible. The central figure is round, instead of being gaping and irregular, and cicatrices from former urethrotomies have become smooth and even.

By means of the endoscope we can diagnose those conditions, of rare occurrence, in the urethra, consisting of polypi or papillomata, and we can also distinguish chancre of the urethra and malignant disease.

\section{TREATMENT.}

The application of remedies is made to the diseased surface by means of a tampon of cotton wrapped around a stick ${ }^{1}$ and introduced through the endoscopic tube. The prineipal drugs in use are: nitrate of silver, from 10 to 100 grains to the ounce; iodine and carbolic acid, equal parts; sulphate of copper, 25, 50, or 100 grains to the onnee; and biehloride of mercury, in 1-per-eent. alcoholic solution.

The intervals of treatment depend upon the amount of reaction; every three days for mild solutions and from five to seven days for

1 The sticks which florists use for tying on to the ends of cut flowers answer the purpose. 
strong ones is about the arerage. After improvement begins intervals should be increased to ten days. The first application should always be mild; 10 grains to the ounce is strong enough to begin with, and it may be increased later if desired.

In regard to the selection of the appropriate remedy, it may be said in a general way that a diffuse hyperæmia calls for milder solutions of nitrate of silver: 5 or 10 grains to the ounce. Granulations require strong solutions of nitrate of silver, from 50 to 100 grains, or carbolized iodine. For erosions mild solution of nitrate of silver, 5 or 10 grains to the ounce, is the best, while for inflamed glands carbolized iodine answers well.

The prostatic urethra requires the strongest applications of nitrate of silver: but under inspection. if the lesions do not improve, the strength of the solutions can be increased or their character changed, as occasion requires.

Polypi and large warts must be remored by surgical means, and the inflamed glands which do not yield to usual treatment can be obliterated by electrolysis.

With the deep form of urethritis, in addition to applications upon the surface of the mucous membrane, we must endeavor by gradual dilatations, with sounds or dilators, to cause the disappearance of the deep-lying infiltrations. 


\section{COMPLICATIONS OF ACUTE GONORRHOEA.}

\section{CHAPTER VI.}

\section{ACUTE GONORRHEA.}

Balanitis is an inflammation of the mucous membrane of the prepuce and glans penis, and is apt to occur in men with a long foreskin, and particularly in persons of uncleanly habits.

The treatment consists in cleanliness, the use of a dusting-powder, and preventing contact of the opposing surfaces of mucous membrane by means of cotton packed between the foreskin and the glans. Occasionally the cdema and swelling are so great that the foreskin cannot be retracted, giving rise to a condition of phimosis.

Phimosis.--This should be treated by prolonged soaking of the penis in hot water and the use of an antiseptic injection thrown in under the foreskin with a flat-billed Taylor syringe. In the event of gangrene threatening or when the swelling and cedema persist, the operation of circumcision or making an incision through the foreskin upon its dorsum is called for. If chancroids are found under the prepuce after operation, they should be cauterized as well as the freshly made wound.

Paraphimosis is that condition in which a long and tight foreskin is caught back behind the glans and cannot be retracted over the head of the penis.

The treatment consists in bringing the foreskin back into its proper place by manipulation. If this prove difficult or if the glans looks gangrenous, the constricting band should be incised on the dorsum, which will generally allow reduction to be effected.

Folliculitis consists in suppuration of one of the urethral follicles, with its retention, forming a small alsscess. Which, if left to itself, opens spontaneously either into the urethra or through the skin. 
The treatment consists in laying the abscess-cavity open freely, as soon as fluctuation is noticed, evacuating the pus, and allowing it to heal by granulation.

Cowperitis is a suppurative inflammation of Cowper's glands, and should be treated in the same way as folliculitis.

Inguinal adenitis is an inflammatory enlargement of the lymphatic glands in the groin, which is generally present in gonorrhœa. The glands rarely suppurate, but usually undergo resolution.

The treatment consists in rest, hot or cold applications, and pressure. If suppuration oceur, the glands should be excised.

Chordee is a complication which adds greatly to the discomfort of a patient with gonorrhea. It is due to the plastic infiltration which takes place into the meshes of the corpus spongiosum, and renders it so rigid and inelastic that it cannot become elongated during erection, but draws on the corpora carernosa, and bends them down, like the tightened string bends a bow.

T'reatment is directed toward preventing these painful erections and allowing the patient to sleep undisturbed. To this end he should use prolonged immersions of the penis in very hot water before going to bed, and should sleep in a cool room, lightly covered. It is better for him to sleep or: the side, as the dorsal decubitus favors congestion of the centre of erection in the cord.

A towel tied around the waist with a knot in the middle of the back will assist in this, for if the patient lies on his back the knot will press into the flesh and waken him.

In serere cases these measures are not enough, and we have to administer sedative drugs. Lupulin, gr. xxx-xl at bed-time; bromide of potash in large doses, 30 grains in the afternonn and again at bedtime, or monobromide of camphor in 5-grain doses will sometimes answer. and, when they do not, we must use opium, preferably by suppository.

When the patient wakes with the penis erect and painful, he should immerse both penis and testicles in a basin of hot water, or, if that is not at hand, cold water will answer the same purpose, and he should also empty his blarder.

"Preaking the chordee" leads to violent hæmorrhage and ultimately to severe tranmatic stricture. 


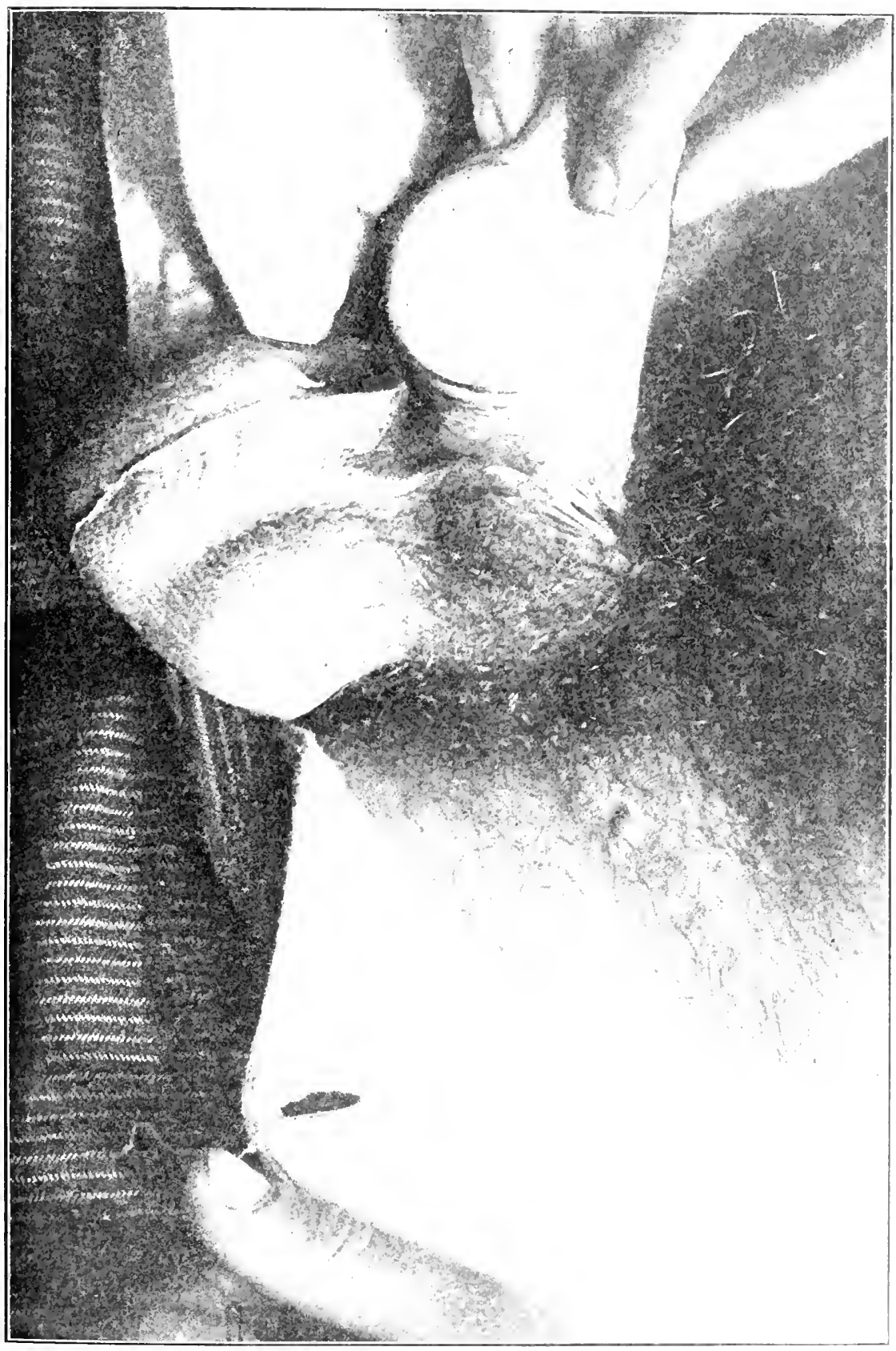



Epididymitis, or inflammation of the hear of the testicle, is not a result of metastasis through the blood-ressels or lymph-channels, but is caused by the passage of gonococci from the posterior urethra to the epididymis by way of the seminal vesicles and ras deferens.

The body of the testicle proper is not involver, as a rule, and the inflammation is limited to the epididymis, but the testicle is often apparently enlarged on account of its being surromded by the swollen and inflamed epididymis. Another element which canses the testicle to appear larger than normal is the eflusion of serum which takes place into the sac of the tunica raginalis, and cause hydrocule. This may, in time, be absorbed or remain permanently and increase.

The symptoms are always well marked. There is serere pain in the testicle, radiating up into the abdomen. The patient's gait is characteristic: he bends over as he stands and walks with his legs straddling in order to relax the spermatic cord and relieve it from the weight of the enlarged testicle.

The purulent discharge of the gonorrhoa generally ceases at once, and remains absent until the inflammation in the epididymis is better, and then the discharge begins again, only not as profusely as before.

On examining the testicle we find the epididymis enlarged, hard, and tender, and the testicle cannot be readily outlined on account of the effusion of serum into the sac of the tunica raginalis. The spermatic cord is thickened and very tender. The inflammatory produets disappear by resolution and rarely suppurate.

If both epididymes have been affected, a condition of steritity may be left, which is permanent. The function of the testicle may be destroyed in three ways:-

I. The most common is the formation of an inflammatory infiltrate in the head of the epididymis, which is not alsonbed, but remains and forms a plug, blocking up the efferent duct.

II. An atrophy of the glandular structure of the testicle may oceur, probably as a result of inflammatory products in its substance.

III. In very exceptional cases the body of the testicle suppurates and sloughs out, and in this way the organ itself may be entirely destroyed.

Tubercular disease of the testicle oceasionally follows a gonorrhœal epididymitis, and is due to the lighting up of a focus of tubercular material, which had been unsuspected and dormant in the epididymis. 
Treatment.--If seen within the first few hours, the severity of the inflammation may be lessened by applying three or four leeches along the spermatic cord. The patient should, of course, be put in bed and the testicles supported by neans of a Curling handkerchief bandiage.

Ilot applications are to be preferred rather than the ice-bag, although ice was at one time very popular. While the ice subdues the inflammatory symptoms promptly, its use is very apt to be followed by a hard, tongh infiltration in the epididymis, which is never absorbed, but remains, blocking up the epididymis and causing sterility. Another disadvantage of ice is that atrophy of the testicle is more apt to occur in the cases where it has been used.

The hot applications can be used in the form of hot lead-andopium wash or flaxseed ponttices, or a poultice made by splitting a

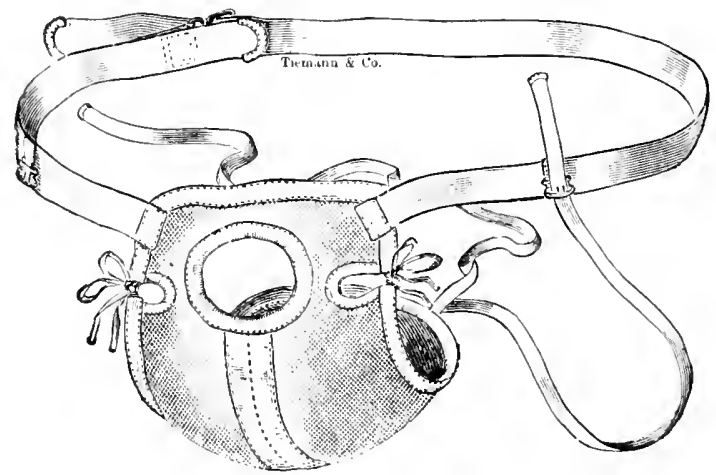

Fig. 23.--Horand-Langlebert Suspensory Bandage.

paper of chewing tobacco, dipping it in hot water for a monent, and then applying it around the testicle. In this way the nicotine passes into the circulation and lessens the arterial tension. In cases where al great deal of effusion has taken place into the cavity of the tunica vaginalis, it is desirable to relieve the tension promptly by drawing off the fluid with an aspirating needle.

In cases of epididymitis which are not very severe, the HorandLanglebert suspensory bandage, lined with a thick pad of cottonbatting, fills the indications of rest, warmth, and suspension, and at the same time permits the patient to walk about without pain.

After the pain of the acute inflammation has subsided Finger 
advises the constant use of eraporating lotions, which he believes are a surer means of causing the absorption of the infiltration in the epididymis than any other, at this particular stage, although later massage is in order.

After the patient is out of bed and begins to go about, the evaporating lotions may be discontinned, and pressure upon the testicle may be applied by means of firm bandaging used in addition to the suspensory bandage.

Various other plans have been used with a riew of promoting absorption of the inflammatory products. A favorite one consisted in strapping the testicle with straps of adhesive plaster, and another which is in common use to-day consists in the application of ointments designed to stimulate absorption. The one which is most commonly employed is:-

R Ichthyol $3 \mathrm{j}$.

I.anolin,

Vaselin aa 3ss.

M. Sig.: To be applied constantly on lint to testicle.

Iodide of potash given internally is of no use in producing absorption of the inflammatory products.

The Paquelin cautery is sometimes used by brushing it lightly over the surface of the skin of the scrotum and huming or searing it very superficially. In the beginning of the disease it has the effect of allaying pain, and later on produces absorption of the inflammatory products.

In order to prevent sterility it is important to promote the absorption of the newly formed tissue in the epididymis.

In recent cases while the infiltrate is soft this may be accomplished by a sort of massage, or kneading, of the infiltration between the thumb and finger; but in cases of long standing, when the infiltration has become hard and dense, all such attempts are unsuccessful.

Gonorrhœal rheumatism is an inflammation of one or more joints occurring in the course of a gonorrhoa and due to the direct action of the gonococcus. The gonococci are carried through the bloodcurrent, and are deposited in various joints, where they set up an inflanmation in the synovial membrane lining them.

If the gonococci alone are deposited in the joint, the resulting 
inflanmation causes an excessive secretion of serum, and a simple luydrathrosis results, or the inflammation may attack the synorial sheaths of tendons and the bursa, occasioning a chronic thickening.

Suppurutive inflammations of the joint are due to a mixed infection; in ardition to the gonococci, staphylococci and streptococci are the exciting causes.

Clinically we can distinguish three forms of gonorrhœal rhennatism:-

(a) IIydrarthrosis, which is usually confined to a single joint (monarticular), and is generally the knee.

(b) Resembling ordinary rheumatism, since it begins with fever, and several joints are involved.

(c) The synovial sheaths of tendons and muscles and the burse are attacked and become the seat of chronic inflammatory changes. The joints may be inrolved or may escape.

The course of all forms is very slow, and recovery is always protracted.

Trealment.-It is of essential importance to treat the gonorrhœa, which almost always affects the posterior urethra or its adnexa.

In the cases which begin with active symptoms-high fever, rapid pulse, and the affection of several joints-salicylate of soda acts well, but in a case which is insidions in its beginning and turns out to be a slow, chronic synoritis, giving rise to a hydrarthrosis, the salicylates are of little use.

Iodide of potash given in increasingly large doses, eren as high as 3 or + drachms a day, is sometimes of more benefit than anything else. Salol and oil of gaultheria are also sometimes of use.

The local treatment is important. The inflamed joint should be put at rest by liecping the patient in bed and applying a light splint to immobilize it. If much heat, redness, and swelling are present, an eraporating lotion or an ice-bag is in order.

After the acute stage has passed off the indications for treatment are:-

\section{To produce absorption of the watery effusion in the joint.}

The most powerful agent, to this end, is blistering, and it should be applied on different parts of the skin over the joint, and used often enough to keep the surface raw for a considerable length of time.

Ichthyol ointment, of 50-per-cent. strength, is sometimes useful as an absorbent. In all chronic cases when the patient walks about pressure over the joint by means of a rubber bandage should be ap- 
plied, and this may serve as a substitute for blistering when the amount of fluid is slight.

When in spite of treatment the fluid does not disappeal, it may be necessary to aspirate the joint, and draw ofl the fluid and irrigate its cavity witls a solution of bichloride of mercury, 1 in 5000. After the eflusion of serum bas been removed the indication is:-

II. To cause absorption of the inflammatery deposit, which has tahen place around bursa, tendons, and synovial sheaths.

This can often be accomplished by massage and the douching or spraying with hot and cold water alternately. Cases selected for this treatment should always be very chronic ones, as much harm would be done to an active inflammation by attempts at passive motion or massige. 


\section{INFLAMMATIONS OF THE PROSTATE.}

\section{CHAPTER VII.}

\section{ACUTE PROSTATITIS. ${ }^{1}$}

THE acute inflammations of the prostate occur in three wellmarked forms:-

I. Simple acute prostatitis, or congestion, which accompanies every casc of posterior urethritis. There is merely an intense hyperæmia of the gland. with a distension of all its blood-vessels. The symptoms are not characteristic, and are only those proceeding from the posterior urethritis.

On rectal examination the prostate is found to be slightly enlarged, soft, and tender to the touch.

The treatment of this condition simply consists in treating the posterior urethritis.

II. The second form of prostatitis is the acute follicular prostatitis. in which one or two prostatic follicles become the seat of an inflammation which is localized to the carities of the glands.

On rectal examination one or two nodules are discernible in the substance of the prostate, which are hard and painful on pressure and which are the inflamed follicles. This condition may terminate in:-

(a) Suppuration, in which the inflammatory material in the follicles supprirates, and breaks out into the urethra, leaving a small fistulous opening which, in the course of time, closes by granulation.

(?.) Resolution, in which the inflammatory products disappear by a process of absorption.

III. Parenchymatous prostatitis, in which the whole substance of the gland is involved in an inflammatory process. This condition may nceur as the resuli of acute congestion or follicular prostatitis. or may derenp independently in the course of a gonorrhcea.

${ }^{1}$ In the con-ideration of the acute and chronic forms of prostatitis no reforence will be made to the senile hypertrophy of the prostate, which occurs in wh men and which is considered later in a separate section. 
The congestion and swelling of the gland reaches its height about the seventh day, and may terminate in either: (a) resolution; (b) suppuration; (c) induration.

\section{SYMPTOMS.}

The symptoms of parenchymatous prostatitis are well marked. The patient has a good deal of fever, and complains of pain in the perineum and rectum, which is increased by pressure. There is a constant feeling as though a foreign body were in the rectum, which occasions expulsive efforts on the part of the muscles and causes reetal tenesmus.

If the enlargement of the prostate is considerable, it bulges into the rectum, and after defecation the stools are flat and ribbon shaped. If the prostate is enlarged anteriorly, it presses upon the urethra and causes diffienlty in micturition or complete retention of urine.

\section{COURSE AND TERMINATIONS OF PARENCHYMATOUS PROSTATITIS.}

In the cases which undergo resolution there is a gradual improvement in the symptoms. On the other hand, if suppuration takes place, an abscess forms in the centre of the gland, and the formation of pus is announced by a throbbing pain in the perineum and chills, followed by fever and sweating. If operative measures are not resorted to, the pus bursts through the eapsule of the prostate and the abscess empties itself into $(a)$ the urethra, $(b)$ the rectum, $(c)$ or through the perineum, $(d)$ or may take an eccentric course.

The pus has been known to burrow its way through the abdominal cavity and finally empty through the inguinal region, the umbilicus, the sciatic foramen, at the edge of the false ribs, through the space of Retzius, etc.

In favorable cases the fistulous opening may close by granulation, but frees, urine, or pyogenic germs may easily enter the fistula, and urinary infiltration, septic infection, and pyæmia are not infrequent results. A perforation into the rectum or urethra may leare a permanent fistulous opening, with its attendant annoyances.

In statistics collected by Segond, in $11 \pm$ cases recovery occurred in 70 and death in 34 . The third termination of induration is rarer than the two preceding. Suppuration does not oecur, and the congestion subsides, but the gland is left in a condition of enlargement and fibrous hardening, which remains permanent. 


\section{DIAGNOSIS.}

Parenchymatous prostatitis may readily be overlooked, and the case regarded as one of posterior urethritis, which is always present and has similar symptoms, unless rectal examination is made, by which the enlargement of the prostate can be easily felt.

\section{TREATMENT.}

The indications are:-

I. To prevent suppuration of the gland.

II. To lessen the severity of the posterior urethritis.

The patient is put to bed, sandal-wood oil is administered, and the pain and tenesmus controlled by opium. Locally either an icebag or hot ponltices are applied to the perineum, a safe guide for the choice between hot and cold applications being the sensation of comfort afforded to the patient.

Continuous irrigation of the rectum with hot water, for half an hour at a time, by means of Kemp's tube is often useful.

If retention of urine should occur, the catheter must be introduced, but only when absolutely necessary, and before catheterizing the urethra should be well irrigated to free it from pus, which might be pushed ahead of the catheter into the bladder.

If great difficulty is experienced in introducing the catheter, it is proper to anasthetize the patient with ether and leave the catheter tied in the bladder, to obviate the difficulty of reintroduction. In extreme cases aspiration of the bladder above the pubes may be demanded.

As sonn as pus forms, the abscess should be immediately eracuated, in order to prevent the pus from bursting through the capsule of the gland, burrowing into the tissues, and causing urinary infiltration and pyamia or at least a fistula which is slow in healing.

Technique of Operation.-The patient is anæsthetized and a sound introduced through the urethra into the bladder. A semilunar incision is made throngh the skin of the perineum, curving around the rectum in order to avoid wounding it. The incision is deepened by dissection until the capsule of the gland is reached and opened and the pus eracuated. The wound is packed and allowed to heal by granulation.

Another procedure which is only applicable to those cases where operation has been delayed and the abscess is seen obviously pointing 
in the perineum is, with the finger in the rectum, as a guide, to thrust a narrow straight bistoury directly into the fluctuating mass, through the skin of the perineum, and evacuate the pus.

\section{CHRONIC PROSTATITIS.}

Chronic inflammation of the prostate may originate as the result of an acute attack of prostatitis, particularly the follicular form. It may also occur from the extension of a chronic inflammation from the posterior urethra, which was either gonorrhcal in origin or due to the chronic congestion and inflammation brought about by sexual excesses or abuses.

\section{PATHOLOGICAL ANATOMY.}

On examination the prostate is found to be enlarged and soft. The crypts and follicles are always attacked by catarrhal inflammation, the cavities of the glands often contain pus, and the openings of their ducts are always large and patulous.

\section{SYMPTOMS.}

As chronic prostatitis is always complicated by chronic posterior urethritis, the symptoms will arise from both conditions, and are as follow:-

(a) Frequent and urgent urination.

(b) Partial or complete impotence.

(c) Mental symptoms, which are grouped under the general term "sexual neurasthenia," and consist of hypochondria, irritability of temper, depression of spirits, inability for prolonged mental effort. forgetfulness, etc.

The only symptom which points directly to the involvement of the follicles of the prostate is

(d) Prostatorrhœa, which is the term given to the discharge of sticky, glairy mucus from the meatus after stool and after urination. It is due to the contractions of the muscles about the base of the bladder and rectum, pressing upon the prostate and squeezing out the contents of the prostatic crypts and follicles through their dilated ducts. 


\section{DIAGNOSIS.}

As the elinical history of ehronic prostatitis, seminal vesiculitis, and chronic posterior urethritis is similar, we can only make a differential diagnosis by a careful examination of the patient.

On passing a bulbous bougie into the posterior urethra extreme tendermess is felt by the patient. The endoscope shows the mucous menbrane of the posterior urethra to be of a deep purple or violet color, bleeding slightly on introducing the tube, and covered possibly with granulations.

After washing out the anterior urethra, if the patient passes his urine in a glass pus may be present or not, and, at the end of the act of urination. Fnerbringer's hooklets, which have been squeezed out from the prostatic erypts, will appear floating in the glass as comma- or tadpole- shaped bodies.

The expression-urine test, which is made by stripping or expressing the contents of the prostate into the urethra, by massage through the rectum. is an important means of diagnosis, as we can in this way get the prostatic secretion for microscopic examination, and at the same time judge of the size and consistency of the prostate itself by the toucli.

Boettscher's crystals are usually found on microscopic examination in the material expressed from the prostate, upon adding a drop of 1 -per-cent. solntion of acid phosphate of ammonia to it.

The crystals are distinguished by their dagger shape and the beautiful forms which they assume upon crystallization. These crystals only form in prostatic fluid, as their base does not exist elsewhere.

\section{TREATMENT.}

It is important, in the treatment of these cases, to seeure the confidence of the patient and encourage him by explaining that his condition is one which can be enred, and that he is not impotent nor likely to become so, and that the mental disturbances of which he complains are due to a purely loeal condition which is not dangerous.

The first indication for treatment is to improve the general condition of the patient by means of diet and exercise in the open air.

Constipation is generally a prominent symptom, and it is necessary to regulate the bowels, using saline cathartics for their depleting action on the pelvic organs, which has some effect in relieving the congestinn of the prostate.

Of coure, all sources of erotic excitement should be interdicted 
on account of their effect in inducing congestion of the prostate. I'nder the head of local treatment we can speak of measures which have a more direct action upon the prostate itself.

Sitz-baths of hot salt-water of from ten to fifteen niinutes' duration have an effect in improving the condition of the diseased organ. Irrigation of the rectum with a Kemp tube, with either hot or cold water, is often beneficial.

The most useful means of affecting the prostate itself is massage through the rectum. We can in this way express the contents of the glands, and after the patient has urinated, if an irrigation is used, the fluid will penetrate into the previously emptied crypts and follicles.

It is important at the same time not to overlook the posterior urethritis, but to cure that as well.

If there is a great deal of eatarrhal inflammation, with much pus-formation, irrigations are in order; but, if the suppuration is only slight, instillations with an Ultzmann syringe are indicated. The endoseope is useful in a small number of cases when granulations are present or when it is necessary to make a strong-application to the verumontanum.

Sounds should be used cautiously and as a last resource, and we should be sure that all acute inflammatory symptoms have subsided,

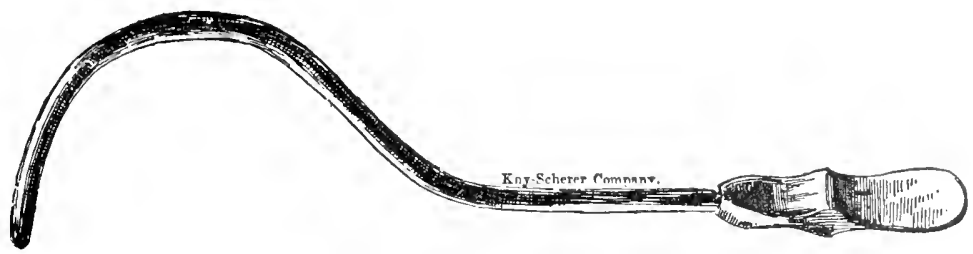

Fig. 24.-Benique Sound.

since epididymitis, vesiculitis, ete., can readily be induced by their reckless use.

The Benique sound is particularly useful here, since it does not tear or lacerate the tissues, and, from its shape and weight, it exercises a certain amount of compression, which is a stimulant to the process of absorption.

The psychrophor, or cooling sound, is a hollow sound which allows a stream of water to flow through it, in order to keep it cold as it lies in the urethra. It is made in the form of an ordinary steel sound, but 
is more nseful if made with the Beniyue curre. The pschrophor combines the eflects of presture and cold, and is often very valuable when prostatorhera is the chicf symptom and occasioned by a relaxation of the mouths of the glands. It should be used every day or second day for from fifteen to thirty minutes at a time.

\section{PROGNOSIS.}

The prognosis of chronic prostatitis is, on the whole, not unfarorable, but improvement is slow, and treatment must be continued for a long time. Improrement can be readily noted by feeling the decrease in size of the prostate through the rectmm, and after a short time under treatment the mental symptoms of the patient undergo a marked change for the better.

Relapses are apt to occur from a lighting up of the catarrhal inflammation and must be treated by suspending all local treatment with sounds and relying on irrigations or instillations of nitrate of silver into the posterior urethra. 


\section{DISEASES OF THE SEMINAL VESICLES.}

\section{CHAPTER VIII.}

\section{ACUTE SEMINAL VESICULITIS.}

ACuTE inflammations of the seminal vesicles are generally due to gonorrhea, although a chronic inflammation may originate from other causes.

A gonorrhøal inflammation of the interior of the cavity of the vesicle is excited when the gonococei pass from the posterior urethra through the ejaculatory ducts, and are deposited within the vesicle.

Its walls secrete pus and its carity soon becomes filled up and distended with a purulent accumulation.

\section{SYMPTOMS.}

The symptoms are not very characteristic, and are merely those of the posterior urethritis, which, of course, is always present. Fever and a throbbing pain in the vesicles and tenderness in the suprapubic region are always observed.

Seminal discharges which are red or chocolate colored from the admixture with blood often occur. The blood may be derived from the cavity of the vesicle itself or may be acquired by the semen, from a congested posterior urethra, as it passes through it.

If the posterior urethritis is cured, the urine is clear at first, but later in the disease the vesicles pour out their purulent contents into the posterior urethra and the pus flows back into the bladder, discoloring the urine when it is passed.

\section{DIAGNOSIS.}

The diagnosis of seminal resiculitis can only be made by rectal examination, and the resicles can be felt swollen, soft, fluctuating, and intensely tender.

Epididymitis is a most frequent result of resiculitis, and is brought about by some of the gonococci-laden pus-cells bein $\underline{n}$ carried through the vas deferens and deposited in the epididymis. 
Peritonitis, which sometimes becomes general, may occur from the close relation which the peritoneum bears to the vesicles.

\section{TREATMENT.}

The treatment of acute resiculitis is chiefly expectant, and consists in putting the patient to bed and giving a saline cathartic, which is always in order in every pelvic inflammation.

Hot sitz-baths and copious irrigations of the rectum with lot water are useful in allaying the inflammation.

If the posterior urethritis is severe, sandal-wood oil relieves the tenesmus and renders the urine less irritating.

Injections into the anterior urethra, of course, are contra-indicated, but above all things any attempt at massage or stripping the resicles should be absolutely interdicted.

Under this plan of treatment most cases of acute vesiculitis will. get well in from two to four weeks' time; but when resolution does not occur the disease passes into the chronic state.

\section{CHRONIC SEMINAL VESICULITIS.}

The condition of chronic inflammation of the seminal vesicles was but little understood until the publication of Eugene Fuller's first paper upon this subject in 1893. Chronic vesiculitis may originate from an acute attack of vesiculitis which does not undergo resolution; but, as a rule, it develops insidiously, as the result of the extension of a chronic inflammatory process which begins in the posterior urethra and extends through the ejaculatory duct. The ejaculatory duct is never compressed by the changes, but throughout the whole course of the disease it remains patulous, and sterility does not occur.

According to the classification devised by Fuller, chronic seminal resiculitis presents itself in two rarieties:-

I. Atonic vesiculitis, in which there is only an atony of the muscular fibres composing the walls of the vesicle.

II. Inflammatory vesiculitis, in which the walls of the vesicles are thickened and indurated as a result of inflammation, which may be simple, gonorrhoeal, or tubercular in origin.

Either form of vesictlitis may exist by itself; but, as a rule, there is a combination of atony and inflammation of the resicular walls. 


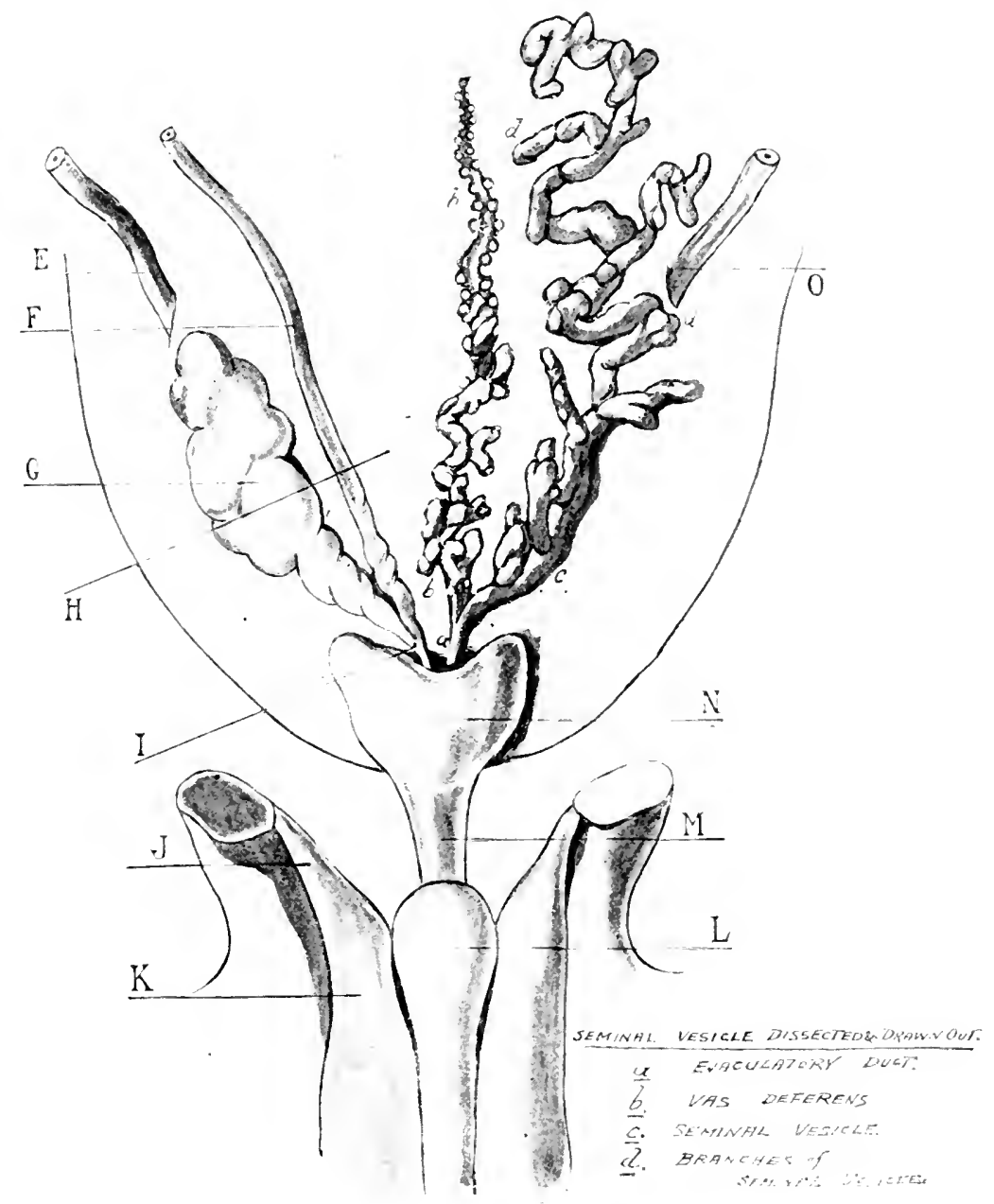

Fig. 25.-Diagram of the Seminal Vesicles. The Right Vesicle has been Dissected, and its Convolutions Drawn Out Straight.

$E$, Ureter.

$F$, Vas Deferens.

$G$, Seminal Vesicle.

$H$, Base of Bladder.

1, Ejaculatory Duct.

\section{J, Crus Penis.}

$K$. Corpus Cavernosum.

$L$, Bulbous Lrethra.

lu, Membranous Urethra.

$N$, Prostate.

$O$, Ureter. 



\section{Atonic Vesiculitis.}

This form of vesiculitis may be induced by a previous attack of acute inflammation in the organ, which does not undergo complete resolution. It is more liable, however, to be provoked by some form of sexual abuse, which consists in a frequent repetition of the sexual act. In consequence the muscular fibres become exhausted, lose their tone, and the walls of the resicles become thinned, atonied, and stretched. The cavities become distended with seminal fluid, because the weakened muscular fibres are no longer able to evacuate the contents, and the secretion becomes thick and inspissated.

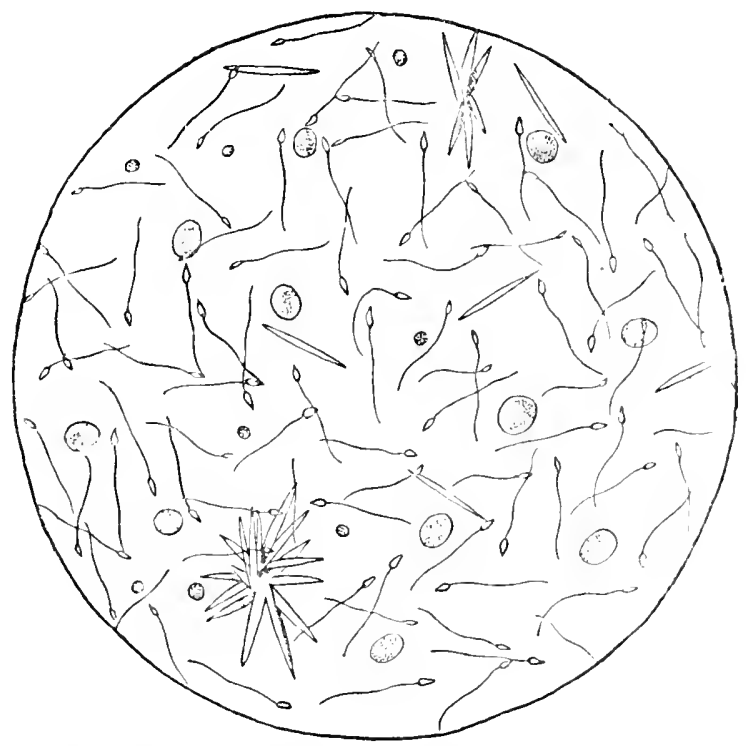

Fig. 26.-Microscopic examination of material expressed from Seminal Vesicles and Prostate, showing spermatozoa, Pus-cells, and Boettscher's Crystals.

The vesicles are distended and enlarged, and feel like a swollen leech to the examining finger.

On stripping the vesicles the expressed seminal fluid appears abnormal.

The expression urine is not clear or slightly milky in color as it should be, but the expressed contents of the resicles appear in the glass of urine as coagulated masses of gelatinous materiue, which often 
resemble casts half an inch long and as thick as a knitting-needle. These masses are the jellified contents of the vesicle, molded by the ejaculatory duets, as they are squeezed through them.

The other form in which the inspissated semen is found resembles sago-like globules floating in the urine.

After a time the atonic vesicle almost invariably becomes infected by some germ. often the bacillus coli from the rectum, and inflammatory changes are induced.

For practical purposes it is only necessary to consider two forms of chronic inflammation of the vesicle:--

(a) Chronic inflammation without perivesiculitis.

(b) Chronic inflammation with perivesiculitis.

When perivesiculitis is absent, there is no inflammatory infiltration or hyperplasia of the connective tissue surrounding the resicles. The resicle is large, and its walls are thinned and atonied, but they are hardened and sclerosed by fibrous changes, as a result of the inflammation which always occurs in this form within the walls.

A muco-purulent secretion may be abundant and accumulate within the carity of the resicle. Granulations often form within the cavity, causing hemorrhage.

The entire vesicle may be filled with a blood-clot, or, if the bleeding is slight, the seminal discharges may be stained brown or chocolate color.

When perivesiculitis is present, a small round-celled infiltration is thrown out in the early stages, surrounding and imbedding the vesicles. In time the infiltration becomes organized with fibrous connective tissue, which forms adhesions, binding the resicles down to the base of the bladder. The entire mass, composed of vesicles imbedded in fibrous tissue, appears like a part of the prostate, and may easily be mistaken for it, on rectal examination.

After the fibrous tissue has existed for a little time, it begins to contract and in consequence the vesicles are pressed upon and squeezed together so that they become smaller than normal.

The ejaculatory duct is never compressed by these changes, but always remains open; consequently there is no sterility.

\section{ETIOLOGY.}

As to the etiology of the inflammatory forms of vesiculitis, gonorrhoal infection is a very frequent cause.

Chronic vesiculitis may originate from an acule attack of vesicu- 
litis; but it is more frequently due to a gonorhcal inflammation of the posterior urethra, which extends along the ejaculatory duct and attacks the walls of the vesicle and often the perivesicular connective tissue.

Other causes besides gonorrhoea may induce chronic inflammatory changes in and around the resical walls.

A non-specific resiculitis, so called to distinguish it from the gonorrhœal, or specific, form, may arise within the cavity of the resicle itself, as a result of an atonic condition of the vesicle.

Again, a low grade of chronic inflammation of the posterior urethra may be established as a result of sexual excesses, masturbation, etc., which spreads along the ejaculatory duct and attacks the resical wall.

Finally, with old men who are beginning catheter life the traumatism often excites a subacute inflammation of the resicles. which extends to the epididymis.

\section{SYMPTOMS.}

The symptoms of both gonorrheal and simple vesiculitis depend chiefly upon the accompanying posterior urethritis, and consist in frequent urination and spasm or irritability of the cut-oft muscle. On straining at stool a glairy, sticky discharge escapes from the meatus, which is the secretion expressed from the prostatic crypts by the muscular efforts, and is termed prostatorrhoca.

The mental symptoms are always very prominent, and the individual suffers from depression of spirits and melancholy, irritability of temper, and quarrelsomeness. Hypochondria is notably marked, and patients are alarmed orer ridiculous trifles. They complain of the penis being shriveled, cold, or numb; that the testicles are retracted or that one hangs lower than the other; and suppose that the testicles are beginning to atrophy and they are growing impotent. In their alarm they consult some of the advertising quacks, who prey upon their fears and deluded ignorance.

Another important group of symptoms is connected with the sexual function. In the early stages there is an increase in the sexual desire, with frequent nocturnal emissions and premature ejaculation on coitus. The seminal discharges are often mixed with blood, which may be acquired from the posterior urethra or the carity of the vesicles. As the case progresses, erections cease. the sexual desire is lost, and at last a condition of true impotence is established. 


\section{DIAGNOSIS.}

The clinical history of these cases is generally significant of involvement of the resicles, in the gonorrhœal form. The patient complains of a gonorrhceal discharge, which lasts, with intermissions, for years. Any slight indiscretion brings on an exacerbation, which is often mistaken for a fresh attack of gonorrhœa. The discharge lasts for a few days and then subsides, until another indiscretion lights it up again.

'The diagnosis can only' be made by rectal examination, and in order to determine the condition of the vesicles a very considerable amount of practice is necessary in order to attain the necessary tactus eruditus. As the vesicles and prostate are continuous, without a line of demareation, when the perivesiculitis is present, it is difficult for the beginner to differentiate them, and, until one is experienced, a diagnosis of enlargement of the prostate is generally made, when the resicles alone are enlarged and the prostate is normal in size.

Technique of Examination.- The patient, with his bladder moderately distended with urine, assumes the "leap-frog" attitude, bending over a chair and grasping the sides with the hands. The surgeon makes counter-pressure with one fist doubled up, pressing against the bladder, and the index finger of the other hand is inserted into the rectum. In order to get well up beyond the upper margin of the vesicle, it is neeessary for the surgeon to support his right foot on a chair and by means of his knee make strong pressure against the elbow of his examining hand, in order to drive it well in.

If the resicles are normal, they feel soft, and the amount of distension depends on circumstances. If atonic resiculitis is present, they feel large, distended, tense, and very painful. If perivesiculitis exists, they are hard, indurated, and brawny, feeling like a piece of pork, on account of the infiltration through the connective tissue, which surrounds the resicles and forms adhesions which bind them down upon the bladder.

After examining the condition of the resicles their contents should be stripped or expressed by the examining finger, beginning at the upper margin and squeezing or stroking in a downward direction, so as to press out the contents through the ejaculatory duct. The quantity of expressed material which runs ont at the meatus varies from a few drofs to half a drachm or more. On examination the material is found to be thick and jellied or purulent, and the 
spermatozoa are dead. As the ease improres, the spermatozoa regain life and motion.

\section{TREATMENT.}

The treatment consists in stripping or expressing the contents of the vesicles once in trom five to seven days. The effects of stripping are to empty the vesicles of their inspissated contents, without forcing the muscular fibres to contraet, and ejeet the semen, and through the rest thus aflorded them the muscles recover their tone. The inflammatory thickening around the resicle is absorbed as a result of the massage.

Contra-indications to stripping are $(a)$ the existence of an acute attack of resienlitis, (b) blood in the expressed material, $(c)$ or exeessive tenderness. With these conditions present there is always danger of setting up an epididymitis.

The posterior urethra should not be overlooked, but should receive appropriate treatment, with irrigations or instillations or by the endoscope. It is desirable, however, not to use loeal treatment to the posterior urethra and strip the vesicles at the same sitting, but to allow a couple of days to intervene. The duration of treatment is protracted, requiring from two to twelve months to effect a cure; but since Fuller's work upon the subject it is possible to eure cases which were berond the reach of treatment before.

\section{TUBERCULOUS VESICULITIS.}

The seminal resicles are usually involved by an infiltration with tuberculous nochles during the progress of a case of genito-minary tuberculosis.

The infection may extend from tuberenlons deposits in the posterior urethra or prostate or may result in consequence of an extension of the disease from the epididymis along the ras deferens.

It is supposed that the involvement of the resicles in the majority of cases is secondary, although J. W. White has noted that the resicles often show evidences of infection weeks or months before the tuberculous process is evident in the corresponding epididymis.

A chronic or subacute inflammation of the resiche, either gonor- 
rhoal or simple, is a strong predisposing element in allowing the tubercular process to become ingrafted upon these organs.

Uncomplicated tuberculous disease of the vesicles is never acute except in the presence of a mixed infection, either with the gonococcus or staphylococcus.

In this case an acnte suppurative inflammation takes place within the carity of the vesicle, which becomes filled and distended with pus.

Incomplicated tuberculosis begins insidiously, and has a notable tendency to invade the surrounding tissues, and the process often becomes quiescent, although liable to take on renewed activity upon slight provocation.

A few cases, however, result in breaking down of the tuberculous nodules, with the formation of a perivesicular abscess, which discharges, either through the rectum or perineum, leaving a fistula.

The symptoms of tuberculosis of the vesicle are not marked, and this condition exists unsuspected until a rectal examination is made, disclosing hard nodules in the walls and perivesicular connective tissue.

As both vesicles are infiltrated and thickened as well as the connective tissue around them, the mass is continuous with the prostate, and it is no easy task to distinguish these structures apart. In adranced cases the prostate is almost always the seat of tuberculous deposits as well.

\section{TREATMENT,}

The treatment consists in hygienic measures alone, of which the most important is an out-door life in a suitable climate. Codliver-oil and creasote are the drugs most in rogue.

Operative treatment is, in general, not required in acute suppurative inflammation caused by a mixed infection, when the accumulation of pus takes place within the cavity of the vesicle, as the pus drains ont into the urethra, through the ejaculatory duct.

When, however, the pericesicular tissue infiltrated with tubercular nodules breaks down and forms an abscess, it is necessary to evacuate the pus. This may be accomplished through a free incision in the perineum, or, as Fuller and Rontier prefer, by thrusting a director into the tumefaction, through the rectum, and divulsing the tissues until an opening large enough to admit the finger is obtained.

Extirpation of the vesicle has been attempted a few times for tuberculosis, but the results are far from satisfactory.

From the inaccessible location of the vesicles, an extensive in- 
cision is required to reach them, and the hæmorrhage is difficult to control.

'The bladder-walls and prostate are generally affecter, and it is difficult or impossible to remove all the diseased material. The wound is slow in healing, and the confinement to bed exerts a bad influence upon the general condition of the patient; so that the tuberculous deposits, which are usually present elsewhere, advance in consequence. 


\section{STRICTURE OF THE URETHRA.}

\section{CHAPTER IX.}

\section{SPASMODIC STRICTURE.}

Sismodic stricture is a pure functional disturbance, without organic change, consisting in a spasm or cramp-like contraction of the cut-off muscle surrounding the membranous urethra.

\section{CAUSES.}

(a) Located in the posterior urethra. An abnormal irritability from excess in coitus or masturbation.

(b) Located in the anterior urethra. A granular patch or stricture. The introduction of a foreign body, such as a sound or bulbous bougie, or a piece of calculus, formed in the bladder and making its way through the urethra, and finally the reflex irritation caused by a narrow meatus.

(c) Point of irritation not in the urethra, but remote from it, as an operation about the anus, fissures or fistula of the anus, and entozoa in the rectum.

(d) Various psychical influences, such as shame or embarrassmient.

The influence of these various points of irritation is carried through the nervous system and causes, as a reflex, a contraction of the cut-off muscle.

\section{ORGANIC STRICTURE.}

\section{DEFINITION.}

A deposit of newly-formed fibrous connective tissue lying underneath the mucous membrane of the urethra and interfering with its dilatability. This fibrous tissue has a tendency to contract and produce a narrowing of the urethral calibre. 
ETIOLOGY.

(a) Inflammation, which is generally due to gonorrhcea, occasions an infiltration of small round cells which, if not absorbed, becomes organized into connective tissue.

(b) Traumatism.-A laceration or rupture of the urethra is healed by the process of cellular infiltration, which is thrown out to a considerable extent and subsequently becomes organized into connective tissue. Traumatic strictures are always notably dense and tough.

\section{PATHOLOGY.}

In gonorrhoa, particularly the chronic form, the mucons membrane of the urethra becomes the seat of an infiltration with small round cells, which extends into the submucous comnective tissue and finally involves the spongy tissue of the corpus cavernosum.

If the infiltration is absorbed, stricture does not form; but if absorption does not take place the infiltrating small round cells become transformed into spindle cells, and these are ultimately converted into dense, retracting connective tissue (scar-tissue). The transformation goes on slowly, and it requires at least one or two years before the soft infiltration has become organized.

The following changes in the tissues result from the contraction of the new fibrous tissue:-

The crypts and follicles of the urethra, which were surrounded by the periglandular infiltration, have been obliterated by the contraction of the fibrous tissue.

If the corpus spongiosum was involved, its meshes are obliterated in the same way.

The calibre of the urethra is narrowed by the presence of a mass of firm, bloodless scar-tissue, composed of fibrillated connective-tissue cells lying underneath the mucous membrane and covered with many layers of squamous epithelial cells.

Desquamation of these squamous epithelial cells continues for years, forming light filaments. which float in the urine.

\section{VARIETIES OF ORGANIC STRICTURE.}

I.-Soft or recent stricture is merely an infiltration of the tissues with inflammatory products, composed chiefly of small round cells. 
II.-Cicatricial or inodular stricture is a mass of new fibrous tissue which has been formed by the transformation of the soft infiltration into true contractile connective tissue, and which, as it becomes older, grows tougher, denser, and more elastic.

A stricture is described as linear when it consists of a fine band of fibres, annular when it is composed of a broader band encircling

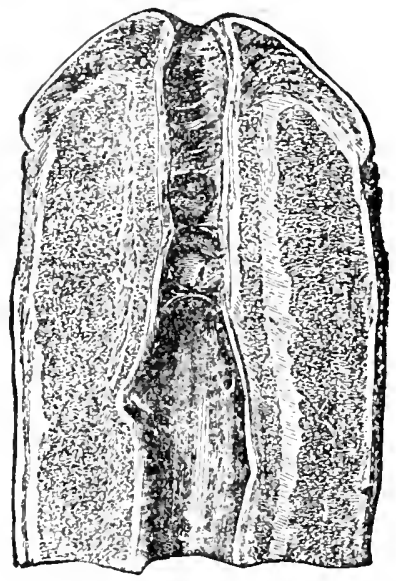

Fig. 27.-Linear stricture.

the urethra, and tortuous when it is made up of a heavy, irregular mass of tissue, producing a great deal of distortion and narrowing of the urethral canal.

\section{NUMBER.}

Trammatic stricture is ahways single, and occurs at the point of rupture in the canal.

Gonorrhceal strictures are apt to be multiple, and it is usual to have two or even three present at the same time.

\section{LOCATION.}

Sir Henry Thompson examined 320 anatomical preparations of stricture and found that in 215 cases the bulbo-membranous region was strictured and in 105 cases the stricture was in the first five inches of the canal.

The prostatic urethra is never strictured except as a result of traumatism, as inflammation here only produces a simple condensation 
and hardening of the submucous tissues, but does not narrow the calibre of the canal.

\section{CHANGES WHICH TAKE PLACE BEHIND THE STRICTURE.}

As a result of the obstruction to the free ontflow of the urine, the urethra becomes enlarged and pouch-like, and a loss of its elasticity follows, from the frequent stretching which accompanics each act of urination.

This pouch retains a drop or two of urine, which decomposes,

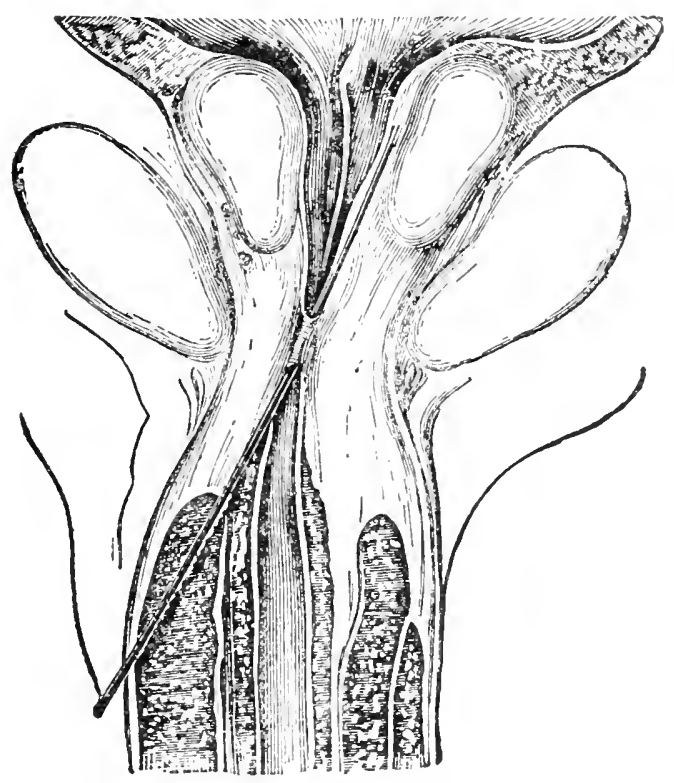

Fig. 2S.-Annular Stricture.

irritates the mucous membrane, and causes a chronic inflammation, with a gleety discharge.

The softened mucous membrane sometimes ulcerates. When a sniall ulceration occurs, a few drops of urine escape into the tissucs and occasion a small abscess, which opens externally, forming a urethral fistula, or the urine may gain access to the tissues through the crypts and follicles which become dilated.

If a large ulceration takes place, a considerable quantity of urine 


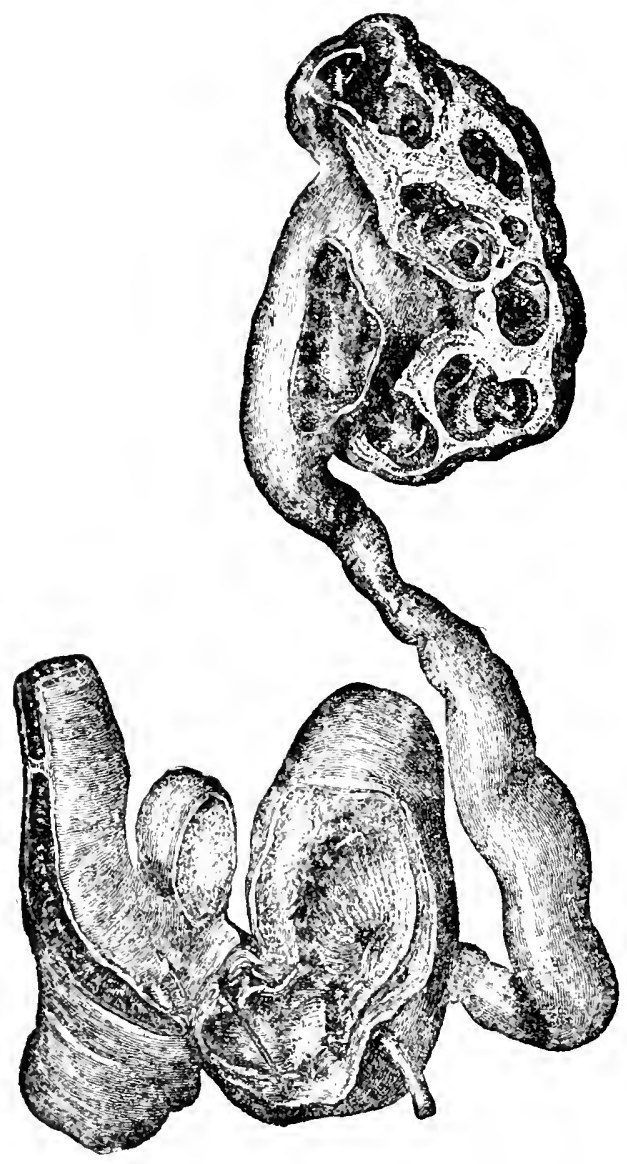

Fig. 29.-Changes behind a Stricture. Dilatation of pouch immediately behind stricture. Hypertroply and Contraction of Bladder. Dilatation of Lreter and Kidney (Hydronephrosis).

escapes into the loose cellular tissue, and extravasation of urine results.

\section{CHANGES IN BLADDER AND KIDNEYS.}

The increased eflort on the part of the bladder to overcome the resistance oftered by the stricture causes a hypertrophy of the bladder-wall. The muscular bundles project into the cavity of the bladder and diminish its capacity, and contraction of the cavity occurs. 
In exceptional cases the walls become thimned and atrophied, and resical atony results.

\section{RESIDUAL URINE.}

Definition.-The urine which remains in the bladder afler the patient has endeavored to eracuate it completely.

Residual urine exists in 93 per cent. of cases of stricture, and increases as the age of the patient advances. It results in cystitis, with vesical atony and damming back of the urine upon the kidneys.

The ureters and kidneys liecome distended and dilated from the backward pressure of the urine, as a result of the muscular contractions of the bladder during urination.

The medullary tissue of the kidneys is atrophied, and sac-like dilatations form (hydronephrosis). Pyelitis and abscess of the kidney occur as a result of infection with bacteria conveyed upward from the decomposing residual urine.

\section{SYMPTOMS.}

(a) Frequent urination, in the early stages depending upon a congestion of the posterior urethra and exaggerated irritalility. Later in the course of the case cystitis canses the desire to urinate frequently, and after the bladder has become atonied and full of residual urine the dribbling and incontinence, or orerdistension, take place.

(b) Dribbling after urination results from some drops of urine, which are caught in the pouch behind the stricture, escaping a few minutes later.

(c) Distorted or Smaller Stream.-The patient requires a longer time to pass his water, and the stream is twisted or split.

(d) Gleety discharge from the meatus, composed of muco-pus and shreds in the urine, is occasioned by the catarrhal inflammation of the mucous membrane behind the stricture, from the irritation of the decomposing urine.

(e) Retention of urime occurs at times suddenly and early from acute congestion of the nucous membrane at the strictured point, and is apt to be excited by exposure to cold or wet and alcoholic or sexual excesses.

Later in the progress of the case the retention is cansed by the direct obstruction of the outflow of mine, arising from the slowly contracting strictmre. 
(f) Pain in the urethra is neuralgic in character and inconstant, and may never be felt.

(g) Interference with Coitus. - The erections are generally feeble, and premature ejaculation occurs from congestion and irritability of the posterior urethra, or the semen may be retained behind the stricture until the engorgement of erection subsides, when it oozes from the meatus.

\section{DIAGNOSIS.}

The cxamination of the urethra, as a rule, is not very painful, but in nerrous individuals it may be injected with a 4-per-cent. solution of cocaine.

For purposes of diagnosis the steel sound is too inexact, since, without disclosing any details, it will merely demonstrate that an olstruction exists in the urethra which prevents the sound from entering the bladder.

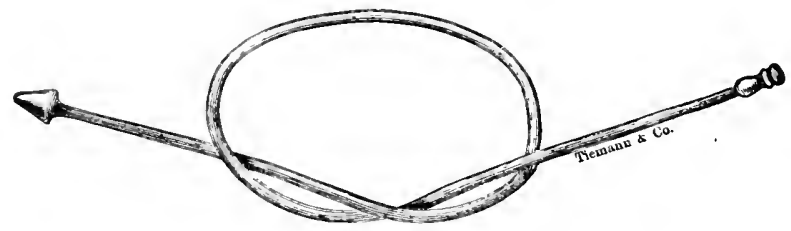

Fig. 30.-Flexible Bougie a Boule.

By means of the flexible bulbous bougie it is possible to feel the slightest pathological changes in the canal. As the bulb is introduced it glides along the urethra for six inches, until it fetches up against the cut-off muscle, and as it passes through the membranous urethra it is grasped by the muscle, but it feels freely movable again as soon as the posterior urethra is gained. When it reaches the sphincter of the bladher it is slightly grasped again, but slips by and moves freely in the eavity of the bladder.

The same sensations of resistance and grasping are felt on withdrawing the bulb, and we should guard against the error of mistaking the resistance of the ent-off muscle for a stricture in the deep urethra.

As the hulb is moved the healthy mucous membrane of the urethra feels solt and velvety, but, when a stricture is impinged upon, a sencation of jolting is felt. There is a perceptible roughness of the walls of the urethra, or one or more fine bands, like fiddle-strings, may 
be discovered. These changes are more easily felt upon the withdraual of the bulb.

The metal bulb is less useful than the flexible bongie, as its smooth, polished surface glides orer the roughnesses of the surface without being held by them.

Fig. 31.-Metal Bougies it Bunle.

The 0tis urethrometer is chiefly nseful in measuring the calitre and determining the dilatability of the urethra. It has the advantage that it can be introduced throngh a narrow meatus and expanded behind the stricture, and we are enabled to measure its ealibre with aceuracy.

In the case of very tight strictures, in which the calibre is too small to allow the passage of the smallest bulbous bongie, we have recourse to whalebone filiform guides.
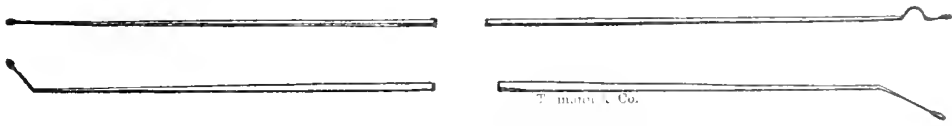

Fig. 32.-Filiform Whalebone Guides.

As long as urine flows ont past a stricture we camnot speak of it as impassable, althongh the difficulty of entering it may be great on account of its fine, narrow lumen or becanse the opening is not central, but lying off to one side.

In order to find the opening it may be necessary to pass six or eight guides down upon the face of the strieture, and, with an assistant holding them firmly in place, try, by tristing and manipulating successively one after another, to find the opening through the stricture.

It is always desirable to inject 5ij of olive-oil into the unctira. 
to lubricate the canal and ristend the narrow opening through the stricture.

Another manipulation, which sometimes succeeds, is to pass a sound down upon the face of the stricture, and hold it pressed firmly for ten to twenty minutes. In this way a certain amount of dilatation is often accomplished, and a funnel-shaped depression in the stricture is formed, with the opening at the bottom, instead of being located off to one side, as before.

In not a few instances the opening through the stricture can be discorered by visual inspection through an endoscope.

If great difficulty has been experienced in introducing a guide through a stricture, it is better not to withdraw it and take the chances of getting it in again, but tie it in, and leave it, either for the purpose of continuous dilatation or for operation.

\section{TREATMENT.}

All strictures, no matter where they are located, if soft and recent, are best treated by gradual dilatation. After the small round-celled infiltration has become converted into fibrillated connective tissue and is dense and contracted, dilatation no longer meets with the success which would have attended its use in the earlier stages. Even in these old cases, howe er, we can in many instances enlarge the calibre of the stricture and maintain it subsequently, by means of an occasional passage of a sound, at a size which does not obstruct the urinary outflow. On this arcount it is always well, in nearly every case, to try the effects of $d_{\perp}$ atation before proceeding to the more heroic measures of operatiois.

In private practice, where patients are more regardful of their health, and seek medical advice early, the larger proportion of strictures are amenable to treatment by dilatation. In hospital practice, on the other hand, patients are careless and neglect themselves, and when they do apply for treatment the stricture is apt to be so extensive and so firm and dense that operation is the only resource.

For purposes of dilatation we have recourse to:-

(a) Flexible bougies.

(b) Steel sounds, with the curve recommended by Van Buren.

(c) Oberlacuder dilator.

Tnlike the operative treatment, we may consider both regions of the urethra together in discussing the treatment by dilatation.

In the case of a stricture, particularly if located in the bulb or 


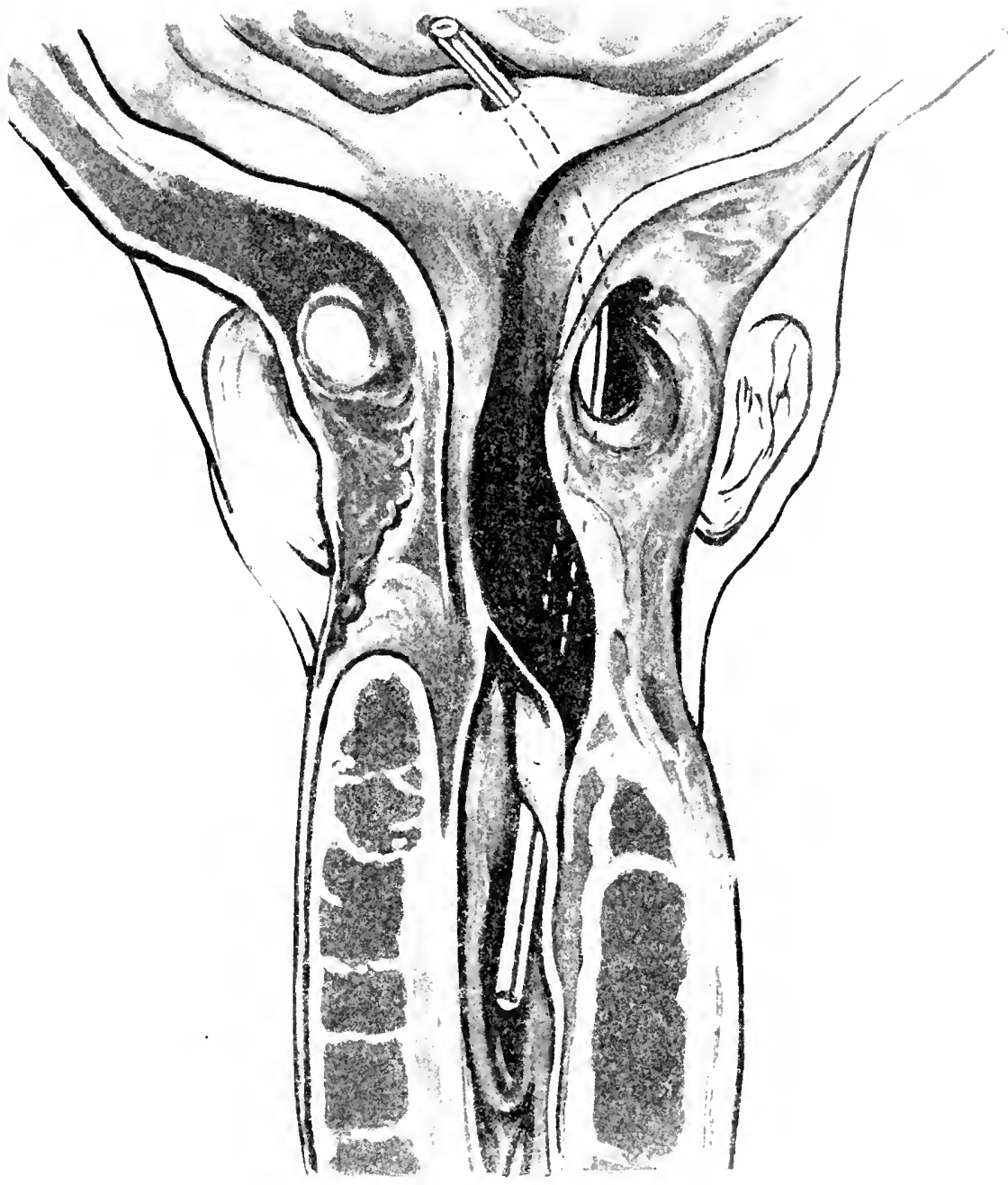

Fig. 33.-Stricture of the Bubo-membranous Urethra and Falie Passage. The instrument has been forced through the tissues into the Bladder. 

membranous urethra, which is below No. 16 French in calibre, we should always begin dilating with an elastic bougie.

If a metal sound is used, there is always danger of lacerating the inflamed and degenerated mueous membrane and pushing the sound into the periurethral tissue, making a false passage. If this aceident oceurs, the point of the sound is felt to be not in the median line, and is grasped firmly by the tissnes, and a finger in the rectum readily detects the deflection of the instrument. Free hæmorrhage from the meatus follows the withdrawal of the sound.

The treatment of such an accident consists in rest, urethral and urinary antisepsis, and the avoidance of instrumentation for three weeks until the laceration has healed.

In using an elastic bongie we should select a size which is small enough not to lacerate the tissues. When the bougie enters the stricture the sensation of its being engaged and grasped is perceived. If it is held very tightly, rather than attempt to push it ahead, it is better to wait a few moments till the spasm has relaxed, and then push the instrument farther along.

After the elastic bongie has been passed through the stricture, it can be withdrawn at once. There is no object in allowing it to remain, since it is compressible and cannot produce any absorption in the stricture; it simply dilates it mechanically. If the instrument has been introduced with comparative ease, and without much pain, we may then introduce another of a larger size.

At the next sitting, which should not take place before two to three days have elapsed, we should first introdnce the number passed upon the former occasion and then use larger sizes.

When the flexible bougie No. 16 French can be introduced with ease, we should begin gradual dilatation with the steel sound. It is important for the beginner to cultivate a suitable technique in using sounds, as mnecessary pain and sometimes actual damage to the tissues are caused by clumsy manipulation. The sound is sterilized by boiling or passing through the flame of a spirit-lamp, lubricated with vaselin or oil, and should be warm.

As the patient lies upon a table the operator stands upon his left side. Sir Henry Thompson advises that for the moment the operator should forget all his anatomical knowledge, and let the somi slip through the urethra by its oun weight, gnirling it with the utmost gentleness, and in no case should any pushing or prodding or force be used. This is well accomplished by holding the sound stationary, in 
a line with Poupart: limment, and drawing the penis up over it until the point of the sound has reached the membranous urethra. The sound is then brought into the median line of the body, and as the point passes through the membranous urethra the handle will of itself describe the arc of a circle and gradually sink down between the thighs and parallel with them. When the handle is completely depressed, it may be rotated freely from side to side, thus demonstrating that the point lies in the bladder and is freely movable.

The largest-sized sound should be selected which will pass through the stricture without using force, withdrawn, and a still larger size introduced. It is desirable, in order to obtain the effect of the proionged pressure, not to withdraw the sound at once, but to leave it lying in the stricture for from five to fifteen minutes.

It the next sitting we should begin with the sound last introduced, withdraw it, and pass the next larger size. The sounds should not he passed too frequently, and the rule is always to wait until the reaction has subsided before passing the sound again. The intervals vary, depending on the amount of reaction, from four to seven days.

There are different riews as to the extent to which it is necessary to carry the dilatation, some authorities claiming that No. 25 French is sufficient, others maintaining that No. 30 French is the proper calibre. In any case there is always danger of recontraction unless the sound is passed at occasional intervals, in order to maintain the maximum calibre, for at least eighteen months. We can introduce the sound every week, and then increase the interrals to once in two, four, six, eight, and twelve weeks.

Effects upon the stricture of passing sounds are twofold, according to Oberlaender and Tossidlo:-

I. The mechanical stretching to which it is subjected.

II. A change in the vital functions of the tissues.

As a result of the distension, small tears oceur in the mucous mombrane or the stricture-tissue, as evidenced by slight bleeding. In a few hours a profuse mucous secretion ocenrs, indicating that a melting of the stricture-callus is taking place in consequence of the reaction. Inder the increased rascularization which sets in, the stricture is partially absolled.

After dilatation to So. 25 or 30 has been aceomplished, the sound only aets upon the norrowest part of the stricture, and when it lies loosely here it accomplishes nothing further in the way of producing absorptiun of the stricture-callus, but only keeps its calibre mechanic- 
ally dilated. But in many cases the stricture-tissue begins to recontract as soon as the use of sounds is discontinued.

Oberlaender claims that by dilating the stricture to more than No. 30 French, even 40 or 45 , the fibrous tissue of the stricture is transformed into a "dead scar," which has no longer any tendency to contract. He states that, by systematic examinations with the endoscope, an evident improvement, which is perceptible to the eye, occurs only after the dilatation of the urethra has exceeded No. 30 French.

When the meatus is of normal size, it will not admit a sound large enough to accomplish this result, and, even if meatotomy is done, the meatus can only be cut to a size sufficient to admit a No. 30 French sound, and this calibre is not enough to accomplish the overdistension of the urethra, according to the views of Oberlaender. On this account he has devised the Oberlaender dilator, which can be introduced, with the blades closed, through a small meatus; after it is in place the blades are separated by turning the screw at the end, and we can get any amount of distension of the urethra which we may desire, even as high as No. 45 French. It should be distinctly understood that forcible dilatation or divulsion is not intended, but only a gradual and temporary dilatation of the stricture. ${ }^{1}$

Technique.-The urethra is cocainized, and the dilator, which is provided with a rubber cover, is oiled and introduced. If the sound which was passed on the case previously was No. 26 , we separate the blades of the dilator to one number higher: i.e., to No. 27.

The blades should be separated very slowly, and after we have screwed them apart one number, as indicated on the dial, we should wait until the pain has subsided, and then increase the separation to the next higher number.

After each dilatation a muco-purulent secretion occurs, which indicates the melting and absorption of the stricture-callus. If the discharge is profuse in quantity, it indicates that the stretching has been too vigorous. The usual intervals for dilatation are about ten days, on the average; and at each sitting the dilatation should be increased from one to two numbers.

The extent to which the stricture should be finally dilated is a matter of individual experience, and beginners generally make the mistake of dilating too rapidly. A sharp bleeding or an excessive

${ }^{1}$ Kollmann's dilator is a more recent instrument, and is to be preferred, as it is provided with four dilating blades, and is less apt to cause a laceration of the urethra than Oberlaender's dilator. 
secretion following dilatation is an indication that an error has been made in the technique, and that the dilatation has been too rapid or too great. If such an accident occurs, it is necessary to wait from three to six meeks, until all tenderness of the urethra has disappeared and the secretion has diminished and is but slight in amount.

In regard to the extent to which dilatation should be carried before the stricture can be said to be cured, Oberlaender states that the encloscope is the only guide. The mucous membrane should show a normal healthy surface and the fibrous tissue should be transformed into a "dead scar," which has no longer any tendeney to contract. As soon as this condition is attained, all further dilatation ean be suspended, quite irrespective as to whether the stricture has been dilated to No. 28 or 30 or to 40 or 45 French.

As with the use of sounds after active treatment has ceased, the stricture should still be dilated, with the dilator, at occasional interrals to prevent relapses.

Relapses sometimes oceur, and ean be recognized by the endoseope and treated with dilatations again, before any decided contraction has had time to occur. For this reason, it is desirable to examine cases with the endoscope at intervals of three or six months after treatment has ceased.

Strictures of very small calibre, which are so tight as not to permit the passage of the smallest flexible bougie, can often be penetrated by means of the filiform whalebone guide. After this instrument has penetrated the strieture and the end has entered the bladder there are three courses open to us:-

(a) Continuous Dilatation. - If a bongie is passed through a stricture which grasps it tightly and is left in place for twenty-four hours, the stricture ulcerates superficially, but widens rapidly, so that the bougie lies loosely within it, and can be withdrawn, and a larger instrument introduced to take its place.

In cases of retention of urine, a guide may be left tied in the bladder, and the urine escapes, flowing away alongside of the guide.

On the following dars larger instruments may be introduced and tied in until the stricture is sufficiently dilated to admit being treated with sounds.

Continnous dilatation. while formerly much in rogue, is now almost olsolete. methods $b$ and $c$ having taken its place.

(b) Tunneled Sound and Gradual Dilatation.-A tunneled sound is threaded over the guide and introduced through the strieture, dilat- 
ing it. The sound is then withdrawn and a larger one introducer in the same way. This is a very useful method of treating tight strictures, which are not too hard and fibrous to admit of gradual dilatiation.

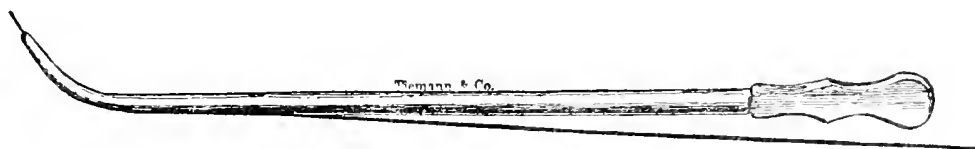

Fig. 34.-Tunneled Sound.

In case of retention of urine, where it is necessary to relieve a distended bladder, Gouley's tunneled calheter can be used in place of the tumneled sound, and the urine withdrawn through it, by pulling out the stylet and thus making the hollow shaft permeable.

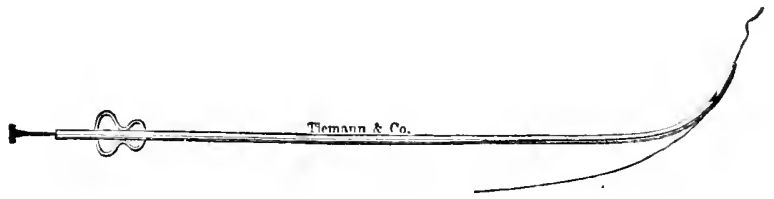

Fig. 35.-Gouley's Tummeled Catheter.

(c) Immediate 0peration. - Internal urethrotomy by Maisonneuves urethrotome or external urethrotomy. (See the following section on the operative treatment of stricture.)

\section{TREATMENT OF STRICTURE BY SURGICAL OPERATION.}

As we have before stated, a soft or recent stricture, no matter in what part of the canal it is located, is best treated by gradual dilatation with sounds or dilators; but, as the stricture becomes olfler, it grows dense, firm, and fibrous, and the gradual dilatation is no longer practicable.

We are obliged to resort to a division or cutting through the stricture-band by means of the knife, and to that end we employ two different operations:-

(a) Internal urethrotomy.

(b) External urethrotomy. 
The cloice of the operation depends eutirely upon the point in the urethra at which the stricture is located, and for the purpose of making the indications for operation clear we can divide the urethra into two regions:-

Region I extends from the meatus backward for a distance of five inches, and its termination corresponds to the peno-scrotal junction.

Region II includes the bulbous and membranous urethra. It extends from a point five inches distant from the meatus back as far as the prostatic urethra.

The operation of internal urethrotomy through the meatus is restricted to Region I, and external urethrotomy through a perineal incision is only applicable to strictures located in Region II.

\section{INTERNAL URETHROTOMY.}

Preparatory Treatment.-Before any operation on the urethra the urine should be rendered aseptic by salol, gr. $x t . i$. $d$, and if strongly acid it should be neutralized by citrate of potash or bicarbonate of potash.

The urethral canal should be irrigated with Thiersch's fluid or salt solution to free it from germs as much as possible.

It is desirable to fill the bladder with Thiersch's fluid by means of a catheter, and leave the fluid in, to flow out after the operation, bathing the cut surfaces and diluting the urine, when the patient urinates later.

Anæsthetic.-A 4-per-cent. solution of cocaine is, as a rule, sufficient, unless in the case of very extreme strictures, which require a general anasthetic.

The technique of internal urethrotomy is as follows: If the meatus is small, it should be incised on the floor to No. 30 French, and any stricture-bands which are within an inch of it should be divided at the same time with a straight, probe-pointed bistoury.

The Otis urethrotome, preferably with Rand's modification, is introduced into the urethra closed. After its point has entered behind the stricture the blades are separated, by means of the screw, to the full extent the calibre of the stricture will allow. The instrument is theu withdrawn until the projection at the end catches against the 
stricture and is held. We know by the feeling of resistance that the projection which conceals the linife lies in close contact with the point we wish to cut. The knife is then withdrawn for an inch, cutting through the stricture, and toward the roof of the urethra. And we

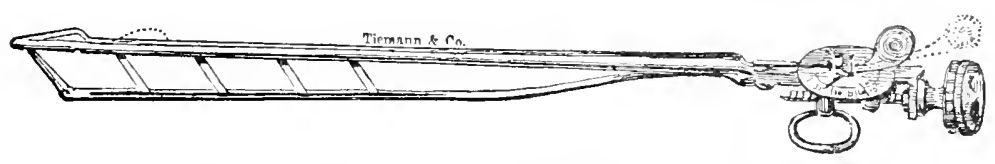

Fig. 36.-Otis Urethrotome, as Modified by Rand.

can afterward readily demonstrate that the stricture has been cut through by separating the blades more widely than was before possible.

The linife is again pushed back into its place of concealment in the instrument, and the urethrotome is drawn out toward the meatus, and any other stricture-bands present are cut in the same way.

In every case treated by internal urethrotomy the question arises: "How deep shall ne make the incision through the stricture?" This is still a point under discussion, since almost every author has his own line of procedure. Dr. Otis derised a scale of measurements of the penis, as a guide to depth of the incision, and claims that a penis three inches in circumference should be cut to admit a No. 30 French sound, while a penis four inches in circumference should be cut so that a No. 38 sound can be passed.

I believe we will have better results by treating each case on its individual morits. The stricture which is comparatively light and does not involve the whole corpus spongiosum may be completely divided, as shown by the entire absence of resistance when the blades of the urethrotome are separated, after cutting the stricture.

In the case of a heary stricture which involves all the corpus spongiosum we cannot divide the whole thickness of the stricture, but must content ourselves with entting it throngh partially, and trust to keeping the channel open by passing sounds frequently for the rest of the patient's life.

After the stricture has been cut to the extent which wc desire and the urethrotome has been withdrawn, a bulbnus hougie. To. 30 or 32 French, is introduced into the canal, to make sure that all bands are completely divided. 
After-treatment.-The patient should be kept in bed and on light diet and directed to drink freely of water and milk to dilute the urine.

In order to keep the cut surfaces from growing together, a straight sound can be passed every day for the first week and subsequently every second day, and, after a month, once a week. Later the passage of the sound need only take place once a month; but there is ahrays danger of recontraction unless a sound is passed at intervals.

Dangers of Internal Urethrotomy.-Nortality. 2 to 5 per cent.

(a) Hrmorrhage.

(b) Infiltration of urine through wound into tissues, producing abscesses and septicemia.

(c) Urinary fever. It is especially important to see that the kidneys are healthy before doing an internal urethrotomy.

(d) Deformities of the penis subsequently.

When a deep cut is made into the roof of the urethra a large amount of scar-tissue is formed in healing. This scar subsequently contracts, and bends the penis into the shape of a bow, which causes erections to be painful, and renders coitus difficult or impossible.

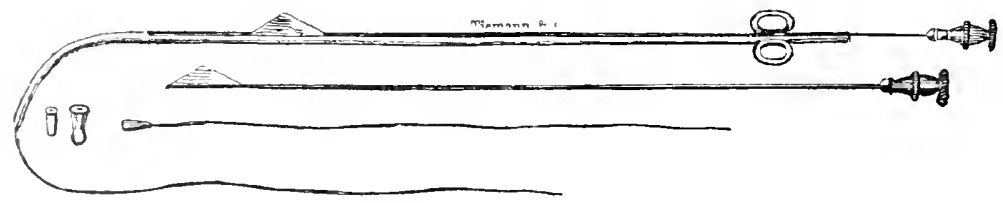

Fig. 37.-Maisomneure's Urethrotome.

Strictures of small calibre, under No. 18 French, are not large cnough to admit the passage of an Otis urethrotome. In these cases a filiform guide should be passed through the stricture and by means of (a) Maisonnenve's mrethrotome the stricture can be divided, from before backward, and afterward cut larger with an Otis uretlirotome, or we can introduce over the guide $(b)$ a divulsor and stretch the stricture so that an Otis urethrotome can be used.

T'he operation of divulsion as formerly practiced has been entirely superseded, as it is inexact and dangerous, producing extersive lacerations of the mrethra. which are followed by hæmorrhage and septic absorption.

Summary of Indications for Internal Urethrotomy.-This operation is apulicable only to stricture of the pendulous urethra which 
is dense and fibrous and cannot be treated by gradual dilatation with sounds, and located less than fire inches from the meatus.

It is especially adapted to the following varietics:-

(a) Distinctly fibrous or non-dilatable stricture.

(b) Resilient stricture. (Definition: Elastic and India-rubberlike, contracting quickly after instrumentation.)

(c) Irritable stricture. (Definition: Standing instrumentation badly and easily excited to inflammation.)

(d) Cases where urethral fever follows each introduction of a sound.

When a stricture is located more than five inches from the meatus. internal urethrotomy is no longer a suitable operation, on account of the danger of hæmorrhage, which is difficult to control. The blood flows backward, orercomes the resistance of the cut-off muscle, and fills the bladder.

The means of controlling the bleeding are as follow:-

(a) Pressure on the perineum by means of a crutch, firmly applied against it.

(b) Introduction of a full-sized catheter into the bladder, which makes pressure on the urethral walls and closes the bleeding ressels.

(c) Perincal scetion and introduction of a catheter through the wound into the bladder, with firm ganze packing around it.

Another danger is infiltration of urine, which soaks into the tissues through the cut and produces absess and sepsis.

\section{EXTERNAL URETHROTOMY.}

In consequence of the dangers of hemorrhage and infiltration of urine, we have recourse to the operation of external urethrotomy throngh the perineum when the stricture is located in the bulbous or membranous urethra, riz.: in Region II.

Forms of Operation.-.(a) With a filiform guide: Gouley's operation, modified by Rand.

(b) Without a guide: Wheclhonse operation.

Gouley's Operation With a Guide.-Technique.-A filifom guile is introduced through the stricture and into the bladder and a tmuneled sound is slid orer the guide, till its point rests acainst the stricture. The urethra is opened in the perineum, by cutting duwn upon the end of the tunneled somnd. 
The sound is withdrawn, the guide being held with forceps, to prevent its being pulled ont of the bladder.

The free end of the whalebone guide is then brought out through

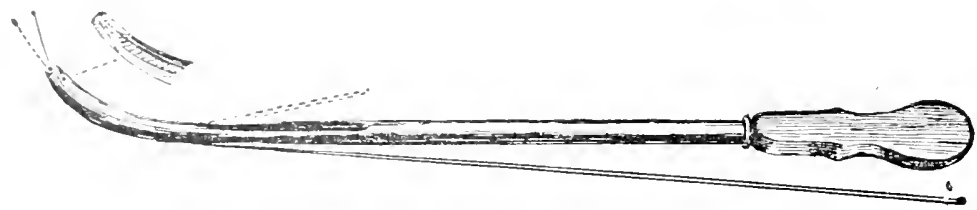

Fig. 3s.-Rand's Tumneled sound.

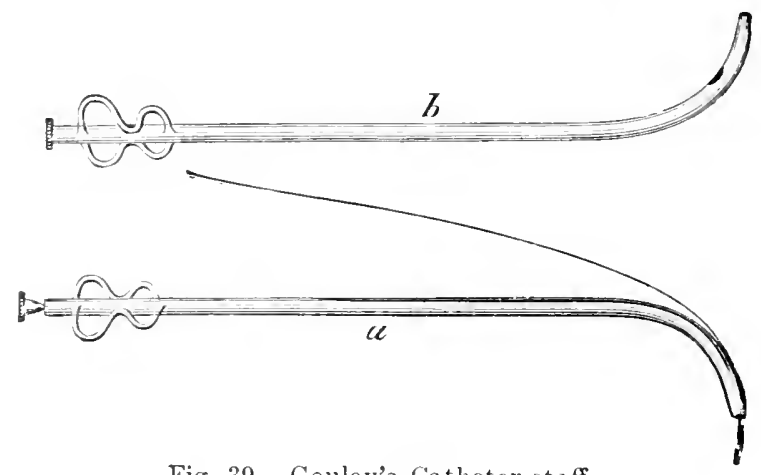

Fig. 39.-Gouley's Catheter-staff.

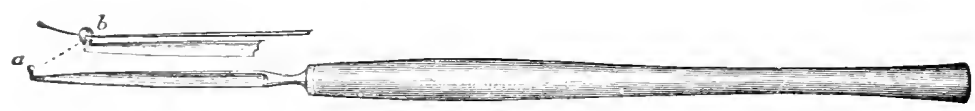

Fig. 40.-Tunneled Knife.

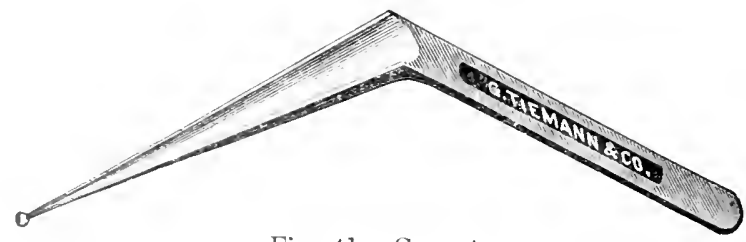

Fig. 41.-Gorget.

the wound (the other end remaining in situ, in the bladder). Rand's tumneled knife is then threaded orer the guide and pushed through the stricture, dividing its fibres. into the bladder. A gorget is introduced into the bladder, and any undivided fibres of the stricture are 
cut with a blunt-pointed straight bistoury, using the gorget as a director.

The operation of external urethrotomy is rendered comparatively simple, if we are able to introduce a filiform guide into the bladter, and it may be impossible to reach the bladder without the assistance furnished by the guide. For that reason it is desirable to perserere in our efforts to get a guide in; when we fail, however, we are obliged to do the operation of

External Urethrotomy Without a Guide, as Devised by Wheelhouse.-Technique.-A Wheelhouse staff is introdneed into the ure-

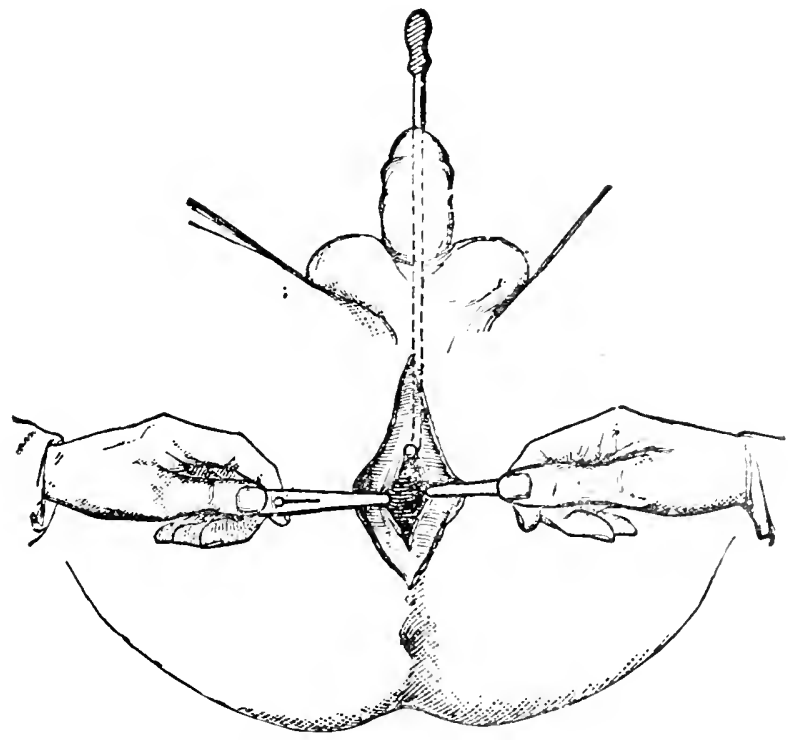

Fig. 42.-External Urethrotomy. Wheelhouse Operation, Exposing the Urethra.

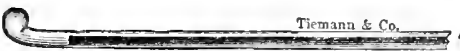

Fig. 43.-Wheelhouse Staff.

thra, till its end rests against the face of the stricture. 'The urethra is opened through the perineum, cutting down upon the end $n f$ the staff. The wound in the urethra is retracted by small hools: on 
wither side, and the upper angle of the wound is held up by hooking the projecting end of the staff against it and drawing it as much out of the way as possible.

Search is then made for the distal end of the urethra by means

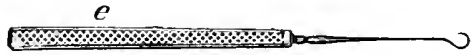

Fig. 44.--Small Tenaculum for Holding Apart Incised Urethra

in External Urethrotomy.

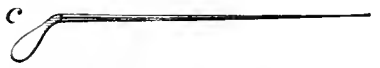

Fig. 45.-Arnott's Grooved Probe.

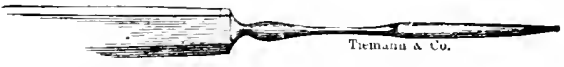

Fig. 46.--Gouley's Beaked Bistoury.

of Arnott's grooved probe or a filiform guide and by inspection. If the opening is found and the probe introduced, a Gouley knife is slipped along the groore in the probe and the stricture is cut through.

In case of failure to find the opening we may have recourse to Guiteras's trocar. The rami of the pubis form a triangle, with the symphysis at the apex. The urethra lies immediately under and onehalf an inch below the simphysis.

If our anatomical knowledge enables us to locate the urethra accurately, by stabbing, in the proper place with the trocar, we will puncture the stricture-tissue, and the trocar will lie in the prostatic urethra beyond, as can be demonstrated by pushing the trocar along through it, until it reaches the biadder, and withdrawing the stylet, when a stream of urine will flow through the hollow cannula. A knife is then introduced along the groove in the instrument and the stricture divided in the usual way.

In the event of this procedure not being successful we may have recourse to retrograde catheterization. This operation consists in making a suprapubic crstotomy and introducing a staff into the bladder and through the prostutic methra and along the canal until its further progress is.barred by the stricture, through which it cannot pass. The end of the staff is felt, corered by stricture-tissue, by means of a finger in the wound. and the tissue which intervenes between the end of the staft and the perineal wound is cut through with a knife, 
in this way bringing the end of the staff into view in the wound. The urethra is thus made again one continuous canal.

We may have recourse to still another procedure, which is to let the patient recover from his anrsthetic and when consciousness is restored and the bladder is full of urine, if the patient endeavors to pass water, a few drops of urine may be seen to escape at one point in the perineal wound, thus indicating the location of the end of the urethra, which had not been discoverable before.

Treatment After External Urethrotomy.-Hamorrhage during the operation is controlled by clamping the bleeding ressels with artery-forceps, which, if necessary, may be left in situ for twenty-four hours in the wound, before being removed. As a rule, this is umnecessary, as the general oozing can be perfectly controlled by firm pressure by means of gauze, packed into the wound and around the catheter.

An important indication is to secure good drainage of the bladder and prevent the urine from coming in contact with the freshly made wound until septic absorption is guarded against by the formation of gramulations. This is readily accomplished by carrying a No. 30 French catheter through the wound and into the bladder, and taking a stitch through both lips of the wound and the eatheter to prevent it from being forced out. The end of the catheter is attached to a long tube, which drains into a bottle on the floor.

In a case where, in addition to cutting a stricture in the deep urethra, a stricture in the anterior part of the canal had been divided

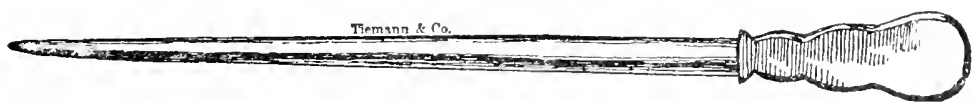

Fig. 47.--Straight Steel Sound.

by internal urethrotomy, a straight sound should be paseed through the meatus and anterior urethra down to the catheter on the second day after the operation and upon every alternate day until the drainage tube is removed from the wound and bladder. The ganze packing should be removed from around the tube in forty-eight hours and the wound irrigated and repacked. It is desirable to irrigate the bladder with Thiersch's fluid every day. About the seventh day after the operation, when granulations have formed, the catheter is removed from the wound and a curved sound passed through the meatus 
in to the blitdder. The sound is passed every second day into the bladler until the perineal wound is healed and the patient discharged.

The patient may be allowed to get out of bed and sit in a chair a week alter the operation, and the ability to hold the water and pass it at will is regained about the same time.

Recontraction of the stricture is almost certain to take place, after dirision of a heary stricture, unless the patient passes a sound, at occasional intervals, for the rest of his life, and this fact should be (arnestly impressed upon him, so that he may not neglect the precaution.

Résumé.-External urethrotomy is applicable only to strictures located more than five inclies from the meatus: i.e., in the bulbous and membranous urethra.

Summary of indications:-

(a) Rupture of urethra.

(b) Urinary infiltration.

(c) Impassable stricture complicated by retention of urine.

(d) Tough fibrous stricture of small calibre which is difficult to dilate.

(e) Traumatic stricture.

\section{MISCELLANEOUS.}

Stricture at the meatus may be congenital or the result of gonorrhoa. In these cases dilatation is useless, and the proper procedure is meatotomy, which is accomplished by cutting the meatus upon the floor by means of a straight probe-pointed bistoury.

There is a tendency on the part of the cut surfaces to unite afterward, which is counteracted by ditating the meatus three times daily with a sound, or preferably a glass cone.

Stricture Complicated by a False Passage.-A large number of cilses of old deep-seated stricture, which have been under instrumentatiun a number of times, are apt to have one or more false passages, which have been made by the bungling use of a sound.

1 false passage adds to the difficulty of entering the bladder with a ratheter or sound, becanse it is very apt to engage the point of the instrument and "pnoket" it.

In these cases the filiform bougie is very serviceable in furnishing 
a guide into the bladder. Our plan of procedure is to introduce one guide after another through the urethra, in hopes that one of them will slip past the opening of the false passage, pass through the stricture, and thus into the bladder.

Combined external and internal urethrotomy is always demanted when both pendulous and bulbous portions of the urethra are the seat of strictures.

It is also often a good plan to open the depp nrethra and drain the bladder with a catheter after an extensive internal urethrotomy, in order to prevent the urine from passing over the fresh cut surface in the urethra and causing urinary fever.

\section{TREATMENT OF INTRACTABLE STRICTURE BY RESECTION OF A PORTION OF THE URETHRA.}

Occasionally dense fibrous strictures of the deep urethra, with a large amount of periurethral induration, are met with, which are sometimes gonorrhoal, but generally trammatic, in origin.

Such patients have usually had their strictures divided by external urethrotomy several times, but, in spite of the regular pasinge of sounds, they contract rapidly, and it is impossible to keep them open.

A conservative method of lealing with such cases is to lay lane the urethra and excise the strictured jortion.

This was first done by König in 1882, who cut out the strictured portion of the urethra and brought the separated edges again into apposition and stitched them together. In 1892 Guyon and Albarran resected strictures and allowed the space between the cut cnds of the urethra to fill in with granulations.

The author has used the method derised by Fuller with sitisfactory results.

Technique.-A sound is introduced through the meatus and the perineal urethra exposed by dissection. If the bulbous urethra is inrolved, the scrotum must be split in two halves in order to lay the urethra bare. The strictured portion of the urethra is entirely cxcised with curved scissors, with the exception of a narrow bridge of urethral tissue one-quarter of an inch wide on the roof. 
A large catheter is inserted at the lower angle of the perincal wound to drain the bladder, and a No. 26 French soft catheter is passed through the meatus and perineal urethra, till its end rests against the perineal tube. The perineal tissues are then sewed around the urethral tube with catgut, thus building up a channel which will eventually form the new urethra.

The skin-edges are then brought together with superficial sutures, and, if the scrotum has been divided, a couple of deep silkworm-gut sutures may be introdnced.

Both tubes may be removed in one week, but a sound should not be passed for three weeks.

\section{EXTRAVASATION OF URINE.}

Extravasation of urine is one of the severest and most dangerous complications which occurs as a result of stricture. It is by no means uncommon, and Sir Henry Thompson found it occurring 8 times out of 217 cases of stricture.

In its attempts at evacuation the straining and pressure of the hypertrophied bladder cause a rupture of the thin walls of the ponch-like dilatation of the urethra, lying behind the stricture, and the urine escapes into the periurethral cellular tissue.

When, as is most frequently the case, the rupture occurs in front of the subpubic ligament, the urine burrows through the cellular tissue of the scrotum and penis and extends upward toward the hypogastrium.

Abscess rapidly forms, the tissues become gangrenous and slough, and spontaneous evacuation of the pus and urine occurs, with considerable destruction of tissue, leaving urinary fistulæ. The septic condition is always rery pronounced, and such patients usually die unless an operation is performed promptly after the rupture occurs.

When the rupture of the urethra takes place posterior to the subpubic ligament, the burrowing of urine takes place in a different direction. In this case the urine cannot make its way forward through the cellular tissue of the penis, but it burrows under the decp layer of the perincal fascia and accumulates in the prevesical space, forning a swelling above the symphysis. From this point it 
extends and inflammatory swelling and suppuration of the connective tissue within the abdomen vecurs and the patient dies of pyamia.

\section{TREATMENT.}

Urinary extrarasation demands immediate operation in order to save the patient's life and prevent extensive sloughing and low of tissue.

An external urethrotomy should be performed and the bladrler drained through a catheter in order to prevent further escape of urine into the tissues. At the same time the collections of pus and urine in the tissues should be opened up, drained freely, and packed with gauze.

If the prostatic urethra was ruptured behind the subpubic ligament and extravasation has taken place into the prevesical space, the pus and urine should be evacuated by means of a suprapubic cystotonny. 


\section{CHAPTER $\mathrm{X}$.}

\section{URINARY FEVER. ${ }^{1}$}

URINARY fever is also spoken of as urethral fever or catheter ferer, and may be defined as a set of symptoms of which chill and ferer are the most prominent, generally occurring in consequence of a traumatism to the genito-urinary tract. It oceurs in two forms: acute and chronic.

Acute Form.- Shortly after instrumentation of the urethra or bladder-i.e., passing sounds, dilatation, urethrotomy, or cystoscopy -a chill occurs lasting half an hour or more and followed by fever, and the minary secretion is lessened or entirely suppressed. After a few hours the temperature falls, with profuse sweating, the kidneys begin to secrete freely again, and the urine contains abundart urates and sometimes albumin.

The chronic form may result as the outcome of an acute attack or may be developed insidionsly without attracting the attention of the patient, who only complains of malaise, dyspepsia, etc. The course of the fever is prolonged, and the patient loses strength and emaciates and is often mildly delirious at night. 'This condition is generally combined with serere cystitis and often pyelitis, and in the end the patient succumbs to the cachexia induced by the chronic poisoning.

\section{PROGNOSIS.}

Crinary fever is a serious disease, especially in old men or with those having diseased kidneys. The most important factor in the recovery of acute cases is the freedom with which the kidneys secrete urine, and, when the kidneys do not resume their function after an acute attack or were previously diseased, the outlook is grave.

\section{ETIOLOGY.}

I'rinary fever was formerly thought to be due to the shock and disturbance to the nerrous șetem, attendant upon passing an instrument into the urethra, and this ricw is still held by many authorities,

"Die Stricturen der Harmiahre," by H. Wossidlo. 
but it is generally regarded at the present time as an infertious disease which is brought about by the entrance of pathogenic organisms into the blood-circulation, through wounds or lacerations of the urethra or bladder. Among various other organisms the bacterium coli commune plays an important part in the cansation of urinary fever.

Micro-organisms are introduced into the blood-circulation in the following ways:-

(a) The sound or dilator may have been dirty and infected with germs, which are introduced into an accidental laceration made in the mucous membrane by the instrument.

(b) The instrument was clean, but the urine was septic and contains micro-organisms from a cystitis or pyelitis, and the attack of fever follows very soon after the first passage of urine, through the urethra. The micro-organisms in the urine pass into the bloot through an accidental laceration in the mucous membrane and occasion the attack.

(c) When instrument and wrine are both sterile, pathogenic organisms may be already existent in the urethra, lying especially in the parts behind a stricture, and are introduced into the circulation through a traumatism.

'The course of the ferer depends upon the size of the wound and the quantity of miero-organisms absorbed. If a small amount of septic material is taken up in oft-repeated doses, the fever runs a chronic course.

In case the kidneys were diseased and incapable of climinating waste-products completely, before the onset of the fever, the attack is more serere, inasmuch as the kidneys fail to excrete the products of nitrogenous waste and, in addition, cannot clear the blood of the aceumulated toxins.

\section{TREATMENT.}

Prophylaxis. - In order to avoid urinary fever the following conditions are necessary:-

I. Healthy kidneys. As already stated, imperfect elimination of urea predisposes strongly to urinary fever: henee it is important to ascertain that the kidneys are healthy before operating on the urethra.

II. Complete asepsis of instruments and genito-minary tract. It is easier to secure asepsis of instruments (see section on care of 
instruments) than to sterilize the genito-urinary tract. A good deal can be accomplished in that direction by irrigations of the bladder and urethra before instrumentation with either boric acid or salt solutions.

The urine should be sterilized by the administration by the mouth of salol, gr. $x$, three times a day, or urotropin, gr. viij, three times a day.

III. All instrumentation should be made with the utmost gentleness to aroid causing lacerations. By avoiding any tearing of the mucous membrane there is no opportunity for miero-organisms to make their way into the blood-current and so excite the fever.

Treatment of the Attack.-As already stated, after the chill the fever subsides, when the patient breaks out into a profuse sweat, and the indication, therefore, is to encourage free perspiration, by putting the patient to bed, surrounding him with hot bottles, and giving a hot drink.

Phenacetin is useful for the headache, and a saline eathartic should be given the next day to complete the elimination of the toxin.

The chronic form of urinary fever is generally accompanied by cystitis, and here the indications are to secure free drainage of the bladder and keep it clean by frequent irrigations. If the inflammation has extended from the bladder upward through the ureters and involved the kidneys, the indication for treatment is to increase the reduced secretion of urine to the normal standard again by means of milk diet and large draughts of spring-water.

Salol and Urotropin should be giren as urinary antiseptics and small doses of quinine are useful as a tonic.

\section{CARE OF URETHRAL INSTRUMENTS.}

All the instruments which are used in the urethra should be perfectly smooth and highly polished, since any roughness upon the surface will abraid the delicate mucous membrane and expose the patient to the dangers of septic absorption and urinary fever. Instruments should also be well lubricated before introduction. Taselin is most commonly employed, but it has the disadvantage of coating the mucous membrane and preventing its contact with irri- 
gating solutions used afterward. Lubrichondrin is a preparation made from Irish moss, is slippery and soluble, readily washes oll, and is preferable to use. Any lubricant shonld be kept in and used from the flexible tubes, which protect it from the air and keep it sterile.

Sounds should be kept in a drawer and prevented from knocling against each other, as that destroys the polish, and after using they should be washed with a piece of ganze in hot water with soap. Afterward they ought to be dipped in aleohol as far as the handle and flamed, or they nay be boiled in a solution of washing soda. ${ }^{1}$ The soda is added to prevent rusting. Urethrotomes, tunneled sounds, lithotrites, silver eatheters, and endoscopie tubes should be serubbed with a brush in hot water with soap and afterward boiled in soda solution. Soft-rubber catheters should be washed off ontside and ruming water allowed to flow through them; but this is not enough for disinfection, and, in order to accomplish this, they must be boiled in plain water afterward.

Flexible gum-elastic bougies and catheters, filiform guides, and rubber-dilator covers and cystoseopes do not stand boiling. They can be disinfected by washing with gauze and soap in hot water, and exposing afterward to the vapor of formalin, in the formalin sterilizer (made by Schering \& Glatz, New York).

A more recent method of sterilizing gum-elastic and soft-rubber catheters now in use in Berlin is to immerse them in the following solution:-

R* Glycerin,

Water $\ldots \ldots \ldots \ldots \ldots \ldots \ldots \ldots \ldots \ldots \ldots$ aa $s$ ounces.

Corrosive sublimate.................... \& grains.

After six hours' immersion laboratory experiments show the catheter to be sterile, and prolonged exposure of the instrument in the solution does not roughen or crack its surfaces.

Cystoscopes may be kept in a jar filled with 5-per-cent. carbolicacid solution, and by keeping them point downward with the eyepiece above the level of the fluid the telescope is not damaged.

Knives with fine, delicate edges are dulled by boiling, but can be sterilized by placing in aleohol or 20 -per-cent. carbolic solution or in the formalin sterilizing eabinet.

${ }^{1}$ Flaming is to be preferred, as boiling roughens their surfaces. 


\section{DISEASES OF THE BLADDER.}

\section{CHAPTER XI.}

\section{CYSTITIS.}

ETIOLOGY.

IT is an event of great rarity for a perfectly normal bladder to become the seat of inflammation, while, on the other hand, any non-inflammatory affection of this organ is most apt to be complicated, sooner or later, by the element of germ-infection, with inflammation ensuing.

There are numerous conditions which may act as predisposing causes to cystitis, although they may exist indefinitely without actually cansing inflammation. The one most frequently met with in practice is the retention of residual urine, occurring in cases of stricture and enlarged prostate or resulting from paralysis of the nerve-supply to the bladder, depending upon a fractured spine or a myelitis. Retention of urine in itself cannot cause inflammation so long as the bladder remains free from infection with micro-organisms; but stagnant urine affords an excellent culture-medium for the growth and development of germs which may be introduced into the bladder, and the retention of urine, if long continued, impairs the vitality of the mucous membrane by keeping it congested.

Chronic congestion of the mucous membrane may occur without the presence of retained urine. and may be occasioned by calculus, some forms of prostatic enlargement, and in women by pregnancy and menstruation.

The normal epithelium of the bladder offers a barrier to the penetration of micro-organisms so long as its cells are intact; but if the bladder-walls are congested for some time, the superficial cells are loosened and desquamated and the softer cells underneath are exposed, allowing the entrance of germs.

The hyperamia also causes the small blood-ressels to rupture, on aceount of the increased vascular pressure.

Exposure to cold is often considered an exciting cause of cystitis, but it can only act in an indirect way by lowering the vitality of the tissues, so that germ-infection may the more easily take place. 
The various predisposing causes above mentioned cannot in themselves excite inflammation. They operate by preventing the bladder from being evacuated and allowing residual nrine to accumulate and form a farorable culture-medium for germs, or by producing congestion of the mucous membrane which lowers its vitality and causes desquamation of its protective cells, thus opening up avenues for infection.

The exciting cause of an attack of cystitis is invariably microorganisms. Normal urine is an ascptic thuid, free from germs, and can be injected into the peritoneal cavity withont cansing suppuration. In every case of cystitis varions forms of cocci and bacteria are always present.

Many of these organisms are incapable of cansing cystitis, if they find their way into a normal bladder, which can be completely emptied of its urine, because they are roided along with the urine, without causing any injury to the bladder.

On the other hand, the staphylococeus pyogenes and the urobacillus liquefaciens septicus and virulent cultures of the barillus coli possess the power of breaking up urea and forming ammonia, and on this account they are able in themselves, without the aid of a favorable predisposing canse, to excite cystitis.

Micro-organisms Found in Cystitis.-C. Mansell Moullin ${ }^{1}$ examined the urine from 30 ases of suppurative eystitis. most of which were old men with enlarged prostates. Cases of tubercular and gonorrhoal cystitis were exchded, and the following results were obtained:-

The reaction was acid or neutral in 24 and alkaline in 6 .

Nicro-organisms were present in abundance in all of them.

The bacillus coli was present in 21 of the acid urines and in 4 of the alkaline cases.

Streptococeus pyogenes accurred in 4 acid and in 3 allialine cases.

The urobacillus liquefaciens was present in $\tilde{\partial}$ of the alkaline cases.

In addition a staphylococens was present in 8 and a cliplococens in 2 .

These examinations show that the bacillus coli is the orginisn

1 "Inflammation of the Bladder and Urinary Ferer," Blakiston, 18.s. 
most frequently present in cystitis when the urine is acid, and the mobacillus liquefaciens septicus when it is alkaline.

Bacillus coli in shape is a short round bacillus with rounded ends which is found normally in the intestinal canal. The virulence of its cultures depends upon the source from which they are taken, a growth from the contents of the healthy intestine having less virulence than one taken from a case of infantile diarrhcea, while a culture from choleraic discharges is virulent in the highest degree.

Unlike other micro-organisms, the bacillus coli has but little eflect in cansing the urine to become alkaline by decomposing its urea, and the urine retains its acid reaction for a considerable length of time. Although the bacillus coli is a harmless saprophyte in the intestine, it has the effect, when injected into the tissues, of causing an abscess or of inducing toxæmia or general septicæmia.

The urobacillus liquefaciens septicus is chiefly of interest from its power of decomposing urea and causing the urine to become alkaline within a few hours.

Cnltures of the bacillus coli, if injected into the bladder, often fail to produce cystitis unless a predisposing cause, such as retention of urine or congestion, be present.

If, however, the urobacillus gains admission to the bladder, cystitis almost invariably follows on account of the ammonia which is set free from the decomposed urea and which acts as an exciting cause for the inflammation.

The streptococcus pyogenes and staphylococcus pyogenes aureus are both capable of inducing septicemia if they gain entrance into the blood-circulation, and locally they attack the bladder-iralls, causing diffuse inflammation with suppuration.

The streptococcus does not decompose urea, and when it is fouml in alkaline urine the decomposition has been effected through the agency of another micro-organism, which is often the urobacillus.

Experimental and clinical evidence serves to show that, while all cases of ordinary suppurative cystitis are dependent upon these (r) similar organisms. inflammation of the bladder cannot be induced by their mere introduction into it, so long as the urine can be completely eracuated and its walls are in a healthy condition. The urobacillus liquefaciens alone is capable of exciting cystitis in a normal bladder, through its property of decomposing urea.

If, however, there be residual urine retained in the bladder, behind a stricture or enlarged prostate, or if the bladder-wall is con- 
gested and eroded from the irritation of a calculus or the administrittion of cantharides, cystitis will surcly result from the infroduction of the other forms of micro-organisms.

Avenues Through which Micro-organisms Reach the Bladder.Germs may enter the bladder by the following routes:-

I. Through the urethra.

II. They may descend with the urine from the kidnoys. bladder.

III. They may pass through the blood-circulation direct to the

IV. They may pass through the lymphatics from adjacent organs.

I. Infection Through the Urethra.-As atready stated, the bacillus coli is responsible for the larger number of cases of cystitis. Its chief habitat is the intestine, where it is always present, although varying greatly in virulence. It is also usually to be found, but mixed with other organisms, upon the glans penis and moder the prepuce and in the fossa navicularis, and in women it exists in the meatus urinarius and the folds of the vulva.

The deep urethra in the male probably does not afford a restingplace for germs while it is healthy. When, however, it is altered by disease, either stricture or enlargement of the prostate, the dilated pouch, containing stagnant urine and mueus, affords a farorable hibernating-place for bacteria.

Cystitis does not occur in men with healthy urethras. except as a result of instrumentation, but in cases of enlarged prostate it is of common occurrence, even when no instruments hare been used. In most instances where the bladder is infected from the urethra a catheter or sound is the means by which the germs are introduced.

Although the instrument may have been sterile before introduction, it may have been infected by brushing against the patient's clothing or acquired germs from contact with the glans penis ol meatus, or it may have carried germs along from a pouch behind a stricture or enlarged prostate in the urethra itself.

But, unless the predisposing causes of congestion of the blathirwall or residual urine be present, the micro-organisms will usually be swept out of the bladder with the first act of urination, without causing any damage.

II. Infection of the Bladder with Bacteria which Desenul in the Urine from the Kidneys. - The bacillus coli, which exists in profusion in the intestinal canal, readily makes its way through the wall of 
the intestine, into the blood-cireulation and notably so, if diarrhoa or intestinal disease be present. After gaining the blood-circulation the hacilli are eliminated by the kidneys, and passing out with the urine gain entrance into the bladder, and under the existence of faroring conditions, such as congestion or residual urine, cystitis is excited.

III. Infection of the Bladder Through the Blood-eurrent. - Infutive emboli ocenring in the course of some general disease may be calried through the capillaries, and local foci of disease in the biadder may be originated through their agency. This cause of infection, however, seldom occurs except as a result of tuberculosis.

IT. Micro-organisms Formed in an Adjacent Organ may be Carried to the Bladiter. Through the Chamel of the Lymphatie Tessels.This form of infection has been proved experimentally and will serve to explain the occurrence of cystitis in women who are the subjects of salpingitis and endometritis and in whom the micro-organisms probably pas from the oraries or nterus to the bladider, through the lymphatics.

\section{CLASSIFICATION.}

Crstitis begins acutely, and frequently becomes chronic. Varions attempts to group the varieties from an etiological or anatomical stand-point have been made, but for practical uses a division into simple. suppurative and specific crstitis serres the purpose.

Tnder the term simple cystitis is understood the inflammation of the bladiler which is not caused by germ-infection, but which results from a mechanical cause. such as the irritation from a calculus or crystals in the urine or from a chemical irritation caused by "antharites. Simple cretitis exists as a theoretical condition only, for, an: a matter of fact, the complication of germ-infection occurs in every race ant in a few hours the bladder becomes infected with micro"leminims, and the simple inflammation is converted into suppurative cystitis.

\section{PATHOLOGICAL CHANGES IN THE BLADDER.}

Location.--The disense process is most marked in the region of the trigone ame particularly so around the ureters and urethral orifier. The fundus is nsully nearly or quite normal.

The mucons membrane is the part nsually affected, but the in- 
flammation may attack the deeper structures and the muscular, subserous, and even the serous coats may be inrolved.

In acute cystitis the cystoscone affords a means of sturlying the changes in the mucous membrane of the bladder. Its color is found to be brilliant scarlet, with branching lines marking the course of the distended ressels, which blced readily. The surface of the mucous membrane has lost its polish, is ragged and relvety, and has flakes of lymph adhering to it. In serere cases the epithclium is detached, leaving erosions.

In very septic cases portions of the mucous membrane sloug? away, and hang from the walls in shreds, and minute abscesses may form in the submucous coat or among the muscular layers. Microorganisms are intariably present; the urine is filled with them and they lie on the surface and between the epithelial cells.

The pathological changes in chronic cystitis resemble those of the acute form, but are more marlied. The erosions are deeper, sometimes forming actual ulcers. The surface of the nucous membrane is black or slate colored, from the escape of blood-pigment into the tissues through small capillary ruptures.

In the early stages the muscular coat may undergo a true hypertrophy of its fibres, but, as a rule, the prolonged inflammation and the vascular degeneration lead in time to a condition of fibroid induration and sclerosis of the bladder-wall. The walls are thicker and denser than normal and their elasticity is entirely lost. Is a result of those conditions the cavity of the bladder often becomes so small and contracted that it can only hold a few ounces. The bladder-wall on contracting falls into folds, with spaces between them, from which it is difficult to empty the urine even with a catheter.

In time the spaces become stretched. forming sac-like dilatations, which may be as large as the bladder itself.

These sacs are only covered by serous membrane, and hare $n$ muscular fibres; so that they cannot empty themselves of their contained urine, and, filled with stagnant decomposing urine, they hecome a farorite seat for stonc-formation.

On the other hand, the bladder-walls, instead of being hard and fibrous around a small contracted carity, may become thin ant flaccid, and the carity may be distended to a capacity of a yrurt or more.

Membranous Cystitis. - As a result of intense scptic infution of 


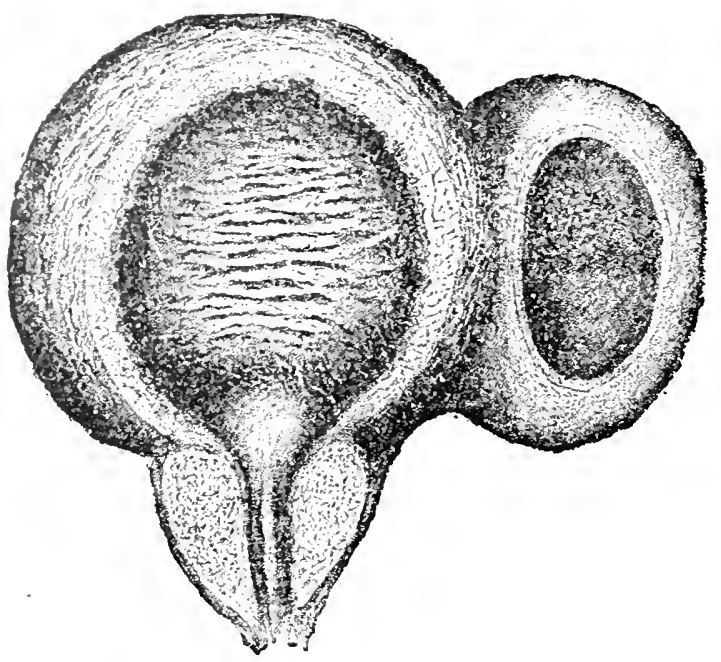

Fig. 48.-Formation of a Sacculation in a hypertrophied Bladder from Prostatic enlargement and prolonged Cystitis.

the bladder. combined with pressure on its blood-ressels, sufficient to shut off the circulation and cause gangrene, a false membrane may form within the bladder, which is thrown off as a coat of its walls.

The microscope shors these coats to be composed of epithelial cells, lymph, urinary salts, and micro-organisms entangled together.

The slough may make its exit through the urethra or the whole thickness of the bladder-wall may slough into the carity of the abdomen.

\section{SYMPTOMS.}

\section{Local Symptoms.}

I. Frequent Urination.-The inflamed and irritable condition of the bladiler-walls and posterior urethra renders the bladder very intolerant of any tension and after a small quantity of urine collects it is expeller. Prolonged standing or jolting increases the desire to urinate in cystitis and particularly so when the inflammation depends upon a resical calculus.

II. Painful Urination.-In acute cases the pain is more or less constantly present orer the bladder, and is aggrarated by the act of urination. As the dusire to urinate comes on, the pain increases, 
and as the bladder contracts the pain may be agonizing, and is telt in the bladder and rectum, and racliates to the end of the penis and down the thighs.

In serere cases there is a desire to minate every few minutas, and the act of urination is accompanied by great straming or tenesmus.

In the presence of stone or posterior urethritis of gonorrhual origin the pain is increased after the act of urination, on accomnt of the inflamed walls of the bladder being squeezed together by the muscular contractions. Strangury is noted in the most severe cases only, in which the pain is continuous and where the patient makes violent and straining eflorts to urinate and only expels a few drops of blood-stained urine each time.

In chronic cases but little pain is experienced, except in the instances of enlarged prostate, when the bladder is hypertropliced and very much contracted.

III. Pyuria.-Pus in the urine is a constant occurrence in every case of cystitis. If the urine is acid in reaction the pus appcars as a cloud distributed through the urine, but if reaction is alkaline and ammoniacal, the pus is coagulated and takes the form of a viscid, tenacious, ropy mass, at the bottom of the ressel.

IV. Hæmaturia.-A slight amount of bleeding oceurs from a rupture of the congested capillaries in the bladder-walls, in all cases of cystitis. The blood is always intimately mixed with the urine and disseminated through it. A gush of pure blood following the act of urination is a characteristic sign of inflammation of the posterior urethra.

\section{Constitutional Symptoms.}

The constitutional symptoms in cystitis are caused by the toxins which are absorbed into the circulation, and their severity depends upon, first, the amount of the poison absorbed and, secondly, upon the rapidity of its absorption.

If the bladder is able to empty itself thoroughly, absorytion does not take place to any great extent and the constitutional disturbance is not marked.

If, on the other hand, the bladder cammot evacuate itself completely or if pyogenic organisms have inraded its walls. serere constitutional disturbance follows.

In acute cystitis the blakher can ustally empty itself, ant the 
temperature is not high and the constitutional symptoms are not farticularly marked; but if, from some cause, obstruction to the ontilow of mine exists, and the protecting epithelium has been desquimated from the bladder-wall, an opportunity for the absorption of toxins is afforded and the constitutional symptoms are grave.

This is particularly true in cases of mixed infection, in which the lacillus coli is associated with the urobacillus liquefaciens septicus or staphylococcus pyogenes.

The ammonia which is formed from the decomposed urea helps to break down the protecting layer of bladder epithelium, and the erosions thus formed allow large quantities of toxins to pass rapidly into the blood-circulation.

In old men at the beginning of catheter-life an asthenic form of cystitis is not uncommon. It is always associated with chronic urinary fever and is often fatal.

In chronic cystitis the constitutional disturbance is slight becanse there is very little absorption of toxins from the bladder. Grave symptoms occur only when an acute attack is ingrafted upon a bladder which has suffered with chronic inflammation. Some organism which is capable of decomposing urea enters and ammonia is formed. The tissues, altered by long-continued inflammation, respond violently to the irritant, the kidneys become involved, and septic poisoning follows.

\section{DIAGNOSIS.}

The symptoms of frequent urination and pain, when accompanied by turbidity of the urine from pus and occasionally blood, are very characteristic of cystitis. The presence of pus or blood in the urine, howerer, only signifies that there is a condition of suppuration sonewhere along the genito-urinary tract. In chronic cases of cystitis it is always important to exclude such local conditions as stricture, stone, or enlarged prostate, and in acute cases the absence of gonorrhoul or tubercular infection should be ascertained and the state of the prostate and seminal resicles shonld always be examined.

The use of the cystuscope is not admissible in acute cases, on accomnt of its increasing the existing irritation, but in chronic cystitis it is indispensable. By its means it is possible to exclude malignunt discase and tuberculosis of the bladder and calculus, and the 


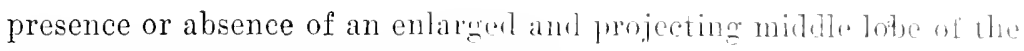
prostate can be readily ascertaincd.

Pyelitis can at the same time be diagnosed or excluded. -nmletimes from the appearance of the meteral openings, but with abo-ilute certainty by catheterizing the mreters.

The microscopic examination is almost as important, to duetrmine the character of the micro-organisms, the rariety of the urinary crystals, and the form of the epithelial cells, from which some conclusion as to the involvement of the kidneys may be drawn.

\section{PROGNOSIS.}

Acute eystitis may heal eompletely or it may become chronic. The cure of chronic eystitis depends entirely upen its eatsation. When it is due to stone, stricture, or enlarged prostate, and its canse can be removed by surgical interference, the prognosis is good.

Chronic eystitis in itself rarely canses death except in the aged and debilitated, who die from absorption of toxins and urinary fever; but, if inflammation of the bladder continnes for some time, the infection travels up the ureters, and the kidneys become the scat of disease which nltimately proves fatal.

\section{PREVENTIVE TREATMENT.}

Suppurative cystitis is always caused by micro-organisms. of which the most common form is the bacillus coli commmis, which originates in the intestine and may make its way into the bladker through the urethra, general blood-cireulation. or lymphatics.

Under ordinary conditions the bacillus coli is a harmless salpophyte, but diarrhea, constipation, and other intestinal diworkrs convert it into an extremely virulent progenie germ. Hence it follows that the intestinal canal should be kept as aseptic as posible by means of calomel, purgatives, and intestinal antiseptics. Local foci of infection, where germs can grow in the nrethra and aromm the external genitals of the male and female, shonld be liept clean and aseptic.

As long as the mucons membrane lining the bladklu remains mbroken and the organ is capable of being completely umption of its contained urine, bacteria may be introduced into the himblum without harm, as they are soon flushed out by the stream of urine, and eystitis can rarely be induced unless the bladuler-watl hats been 
congestee and the epithelial lining eroded or it contains residual urine, eaused by an enlarged prostate, stricture, or vesical atony. On this acconnt it is important to attend to any local diseased conditions which interfere with its proper emptying, and allow residual urine to aceumulate or the bladder-walls to become congested and eroded.

Citheters and sounds must be sterile before using, and the cxternal genitals should always be eleansed before instrumentation (see section on asepsis of instruments).

\section{GENERAL TREATMENT OF ACUTE CYSTITIS.}

In all but the mildest eases the patient should be kept in bed until the severity of the symptoms is controlled and the acute stage is past.

The room should be maintained at an even temperature, for the congestion of an inflamed bladder is notably increased by chilling the surfaee of the body and by the museular efforts of walking.

Purgatives.-A brisk ealomel purge should always be administered at the begimning of an attack for the purpose of elearing out the intestine, which is the main source from which the bacillus coli is derived. It has been shown experimentally that reetal obstruction is almost immediately followed by the appearance of enormous numbers of colon baeilli, which come either from the kidneys or directly from the rectum to the bladder through the thin intervening walls. 1)uring the progress of the case a daily movement of the bowels should be secured by Hunyadi or Rubinat water.

Hot sitz-baths at a temperature of $100^{\circ}$ or $105^{\circ} \mathrm{F}$. are serviceable in allaying the vesical irritability and tenesmus. The exposed part of the patient's body shonld be well covered with blankets while taking them.

The diet should be light and largely composed of milk. Meat should not be allowed at all or only in small quantities. Fresh fruit may be taken in moderation. Alcohol is interdicted unless perhaps in old men who need a stimulant, in which case whisky well diluted may be used.

Diluents. - Pure spring-water or distilled water may be taken freely, unless the desire to urinate is very frequent and urgent. The various infusions of triticum, buehu, ete, probably render the urine hand, simply through the water they contain. Alkalies should never he arministered as a routine measure, since in cystitis the urine is frequently allialine from deeomposition of urea into ammonia or from 


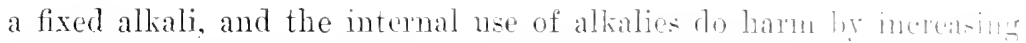
the alkalinity. If the urine is highly acill and deposits unc-iteid colstals, bicarbonate or citrate of potash is nsetul in allaying the incitatinn.

Opium is often required in acute cases to control the resical tumemus, pain, and irritability. It is given preferably in $1 / 2$-grain morplia suppositories, but may be used by the mouth as well. It is munire to inject morphia into the blatder. If the bladder mucous munbrane is morolien, it will not be absorbed, and, if erosions are lrescut, absorption may oceur too quickly, and give rise to symptoms of poisoning.

The same holds true of cocaine, which has little or no eflect in causing local anæsthesia of the bladder, as it does in other mucous membranes, and has the great disadrantage of very materially increasing the congestion of the mucous membrane. at times sulliciently to cause retention of urine.

Balsams.-Sandal-wood oil has a very beneficial action in allaying the too frequent urimation and pain of cystitis, in its acute stage. Later in the attack, when the secretion of pus has diminished so thit the urine, instead of being turbid, presents only a fine clondincs, the oleoresins, such as turpentine, copaiba, cubels, and thuid extract of pichi, and minute doses of cantharidin dissolred in alcohol, have an effect in quickly causing a cessation of the suppuration and a clearing up of the urine.

Urinary Antiseptics. - The antisentics are supposed. throush their germicidal properties, to destroy the inflammatory proeess at its root. Salol may be considered as a representative of the group. It is given in doses of gr. $x$ three times a day, and acts promptly as a thstroyer of miero-organisms, through the constituent carbolic aciel, which, from its decomposition, is set free in the urine. In the same way boric and benzoic acids, in doses of 20 grains per day, extecice their germicidal power, and are to be chosen when the urine is allialine in reaction from the presence of a fixed alkali.

In the gromp of aniline derivatives may be mentioned mathylblue and Trotropin. Iethyl-bluc, first recommended by Einholu, in the quantity of 15 grains a day in tablets or capsules. is olten of service when the urine contains large quantitice of bacteria. The nune from using this drug, becomes greenish at first in color and latur wl an ittense blue. Urotropin, which discolves phospluatic eomoretions and causes phosphaturia to disappear with raphlity and when furmat-

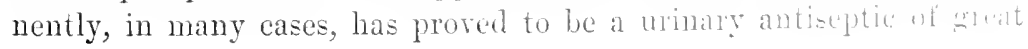


value, and particularly so in cases of ehronic cystitis aecompanied by ammoniacal decomposition of urine. It does not destroy the microorwanisms, but prevents their growth and development. The proper dose is $2 t$ grains per day; if this is exceeded, burning in the urethra and frequent urination oecur.

\section{LOCAL TREATMENT OF ACUTE CYSTITIS.}

Bladder-mashing, which is so essential in ehronic cases, is entirely inadmissible in the aente forms. The only rarieties of local application which ean be used without doing harm are:-

Instillations of Nitrate of Silver.-These ean be used with advantage in the most acute eases of cystitis. The principal indications for their use are painful and frequent urination, prorided the bladder is capable of emptying itself. By means of an Ultzmann syringe 20 drops of nitrate-of-silver solution, inereasing in strength from gr. $j$ to gr. $x$, are deposited every second or third day in the posterior urethra, from which point it flows back into the bladder and triekles over the surface of the trigone, which, together with the posterior urethra, are the parts most involved in the inflammatory process. The bladder should be empty, before the instillation, as the nitrate of silver is neutralized if it comes in contact with urine. Instillations are mainly useful in acute cases, although sometimes of service in chronic cystitis.

\section{GENERAL TREATMENT OF CHRONIC CYSTITIS.}

After the serere pain and frequent urination of the acute stage have passed oft, the urine still contains pus in large quantities and has to be voided more frequently than in the normal condition.

The patient should no longer be confined to bed, but should have the benefit of the fresh air, although much walking is, of course, ont of the question for him. He should be instructed to elothe the body in Hannel, to wear woolen stockings, and India rubbers in damp weather, and eautioned particularly to aroid exposure to a draft or dampness.

The sandal-mood oil is of less use in chronic cases than in the acute, but the oleoresins are of rahe; and the urinary antiseptics, Lrotropin and salol, have here their field of greatest usefulness. By mrans of the cencral treatment but little can be accomplished, and the main reliance is the loeal treatment. 


\section{LOCAL TREATMENT OF CHRONIC CYSTITIS.}

Indications.-I. Remove any local source of irritation within the bladder or any obstacle to its evacuation. P'alliative measures for the cure of cystitis are of no avail if a calculus or tumor be alluivel to remain within the bladder, or a stricture or enlarged prostate causes residual urine to accumulate.

Indication II. Remove the urine from the bladder and keep it empty.

In cystitis, if the bladder is able to empty itself completely, the micro-organisms are soon swept away and the attack is over; but, if residual urine is allower to remain and become stagnant, it affords an excellent culture-medium for the growth of the germs.

The simplest form of drainage of the bladder is by means of a soft-rubber catheter passed once a day or oftener, as needed; but in atonic bladders, or severe cases, this is not sufficient; but the bladder must be kiept empty.

Permanent catheterization may be employed by passing a catheter into the bladder throngh the urethra and tying it in, so that the urine is voided as fast as it is secreted by the kidneys. The disadvantages of this method are that after a few days it is apt to excite inflammation of the urethra, epididymitis, urinary fever, or suppression of urine. In consequence of these drawbacks, except in very light cases of cystitis or in the presence of debility which is so great that there is danger in giving an anrsthetic, it is best to drain the bladder through a perineal or suprapubic incision.

Perineal Drainage.-Technique of Operation.-A lithotomy-staff is introduced into the bladder through the urethra and an incision is made through the perineum, into the membranous urethra, by thrusting a long, straight bistoury three-fourths of an inch in front of the anus, until its point strikes the groove in the staff. A gorget is introduced into the bladder through the wound and a No. 30 French, softrubler catheter carried along it, till its eye lies within the carity of the bladder.

A silk suture is then passed through the skin of the perineal wound and the catheter, in this way keeping the catheter from being forced out by the contractions of the bladder.

Instead of a rubber catheter, Watson's silver perineal drimatetube may lue used in cases where the blarder is tolerant; but the metal tube is apt to excite more pain than the soft-mbher catheter.

The catheter is attached to a rubber tube whose cont lies in a 
bottle on the floor to receive the mine. In this way the bladder is kept entirely ampty, and can be thoroughly cleansed by irrigations.

The length of time during which drainage is to be maintained raries greatly, depending upon the degree of the cystitis. It should be continued until the mine is free from pus and acid in reaction and the muscular walls of the bladder have regained their tone, and this, in a severe case of long standing, may require some months.

Sumalulic drainage is to be preferred to drainage through the perinemm, when it is continued for some length of time, as the flow of urine throngh the abdominal fistula can be received in a rubber bag under the patient's clothing (the Bloodgood bladder-drain) and he can go aljout and lieep dry.

The operative treatment is resorted to only in particularly severe cases, which on account of extreme tenderness make all local means of treatment impossible. Such instances occur in patients with contracted bladders and but little residual urine. They suffer from constant pain and frequent and painful urination, with the discharge of a few drops of ammoniacal urine each time. Again, in inveterate cases of chronic cystitis, where the bladder is atonic and perhaps sacculated, and where bladder-washing and instillations have been used withont effect, permanent drainage through a fistula often causes a marked improvement.

Indication III. Destroy micro-organisms or check their growth and remore pus and fermentation products from the bladder by means of irrigation of the bladder. Washing the bladder is the most efficient mode of cleansing the bladder-walls of adherent pus, micro-organisms, and urinary salts. The micro-organisms which lie beneath the mucons membrane camnot be reached, but, by lessening the congestion of the mucous membrane and remoring decomposed urine, it is put in a condition which farors its recovery.

Aluhongh the bladker can be filled by hydrostatic pressure from an irrigator, it lequires a catheter to wash it out. The only suitable forms of catheter are the soft rubber and the gum elastic. They should be of lare size and provided with two eyes, as the stream flowing thrnugh them stirs up the stagnant urine more effectually if it enters the biakler from two directions.

A large hand-rinblier syinge holding five ounces may be used, and has the advantage that it is possible to judge of the extent to which the bladter is fillod ly the feeling of resistance, to the inflowing stratu, otfered by the mmecular walls of the bladder. The successive 
jets entering also set up contractions of the muscrlar walls, which have an influence in restoring their tone.

The general custom is to use an irrigutor raised to the height of two to four feet and attached to the catheter by a short glass connecting-tube. The patient should rest upon a table or sofa in a half-sitting position, and the anterior urethra shonld be irrigated to cleanse il, before the catheter is carried into the blarler.

As soon as a sense of resistance is perceived or the patient fects pain, the inflow should be stopped, and after a short panse the thuid be allowed to flow ont again, and the proceeding repeated until the solution comes away clear. The amount of fluid necessary to flow in at one time is small: 60 to 150 grammes (from 2 to 5 ounces) is enough.

\section{SOLUTIONS FOR WASHING THE BIADDER.}

For simply cleansing the walls of the bladder and removing adherent pus and decomposing urine, normal salt solution, 0.6 per eent., or boric-acid water, 4 per cent., in strength, are well adapted to the purpose. Salicylic aeid, 3 parts to 1000 , has some effeet in checking the fermentation process.

On aceount of the bacterial origin of the cystitis, many varions antiseptics have been recommended, but on more extended trials they have proved disappointing and have fallen into disuse.

Nitrate of silver is an exception to the antiseptics just mentioned. and is the most valuable remedy we possess in suppurative eystitis. It should be used in the strength of $1-4000$ up to $1-1000$ begimning with the weaker and gradually increasing. The solution should be retained in the bladder two or three minutes and then allowed to flow out again. If much pain is caused it may be neutralized by washing afterward with salt solution. The application should be made every two to three days. Permanganate of potash is less neeful than nitrate of silver in cystitis, but is oecasionally of service.

In chronic cystitis, when the bladier is contracted and much pain is complained of, the distension attendant mpon washing the hidhler contra-indicates its use, and instillatioms of nitrate of silver have to he

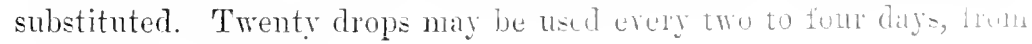
gr. $\mathrm{v}$ to $\mathrm{x}$ in strength. 


\section{SPECİFIC CYSTITIS.}

\section{GONORRHEAL CYSTITIS.}

Statistics show that the posterior urethra is, involved in 80 per cent. of the cases of gonorrhœa, but a true cystitis arising from infection of the bladder mucous membrane with gonococci is extremely rare, although a few cases have been recorded.

Inflammation of the trigone of the bladder frequently occurs during the course of a gonorrhcea, after the posterior urethra has been attacked, and is almost always due, not to the entrance of the gonococci into the bladder, but of some other pathogenic organism, and the infection is therefore of the mixed variety.

Its symptoms, course, and treatment are those of the ordinary forms of suppurative cystitis, and have already been considered under that heading.

\section{TUBERCULOUS CYSTITIS.}

Tubercular involvement of the bladder occurs most frequently in young adults between the ages of 15 and 40 , although no age is exempt.

\section{MODES OF INFECTION.}

Primary infection of the bladder is of rare occurrence, although it is met with occasionally, for. so long as the vesical epithelium is intact. it affords an efficient barrier to the entrance into the tissues of any tubercle hacilli which may have found their way into the bladder. If the mucous membrane has been injured or eroded by the action of ammoniacal urine, the tubercle bacilli can easily enter the tisnes. For this reason primary tuberenlosis of the bladder is rare, and when the bladder becomes tuberculous it is usually infected from a denosit elsewhere.

scemdary infertion of the bladder may result from the direct extension of a tulierenlous infiltration of the seminal vesicles or prostate or the bacilli may be conreyed by the lymphatics or blood-circulation from the lidneys or testicle. Most of the cases of bladder tubereulosis, howeter. originate from the mural transplantation of tubercle bacilli contained in the urine from a tubercular kidney. 
PATHOLOGICAL CHANGES.

The tubercular deposit always begins around the vesico-urethral orifice and trigone or around the wethral openings. The process does not last long as a pure tubereulosis, for it is soon complicated by infection with other micro-organisms, causing suppurative cystitis and ammoniacal decomposition of urine.

A cystoscopic examination made early in the case shows a few minute papules or pin-head-sized ulcerations, and later in the disease are to be seen irregular-shaped ulcerations corered with a deposit of urinary salts and sloughing material, and the base of the ulcer may be covered with fungating granulations, which bleed easily.

The walls of the bladder in some places become hard and rigid and in others are softened, and its capacity is diminished, so that it may only hold a few ounces of urine. The cellular tissue surrounding the base of the bladder becomes the seat of multiple abscesses, which break and form fistulæ.

\section{SYMPTOMS AND COURSE.}

Tuberculosis of the bladder begins insidiously, and often without any apparent cause. Its symptoms are those of eystitis, viz.: frequent urination, pyuria, and hæmaturia. Bleeding is a prominent symptom, appears early in the disease, and is more or less persistent throughout its course.

In the later stages, after suppurative cystitis has made its appearance, the striking symptom is the painful and frequent mination, occasioned by the contraction of the cavity of the bladder and the ammoniacal urine coming in contact with its ulcerated walls. The endeavor to rid the bladder of the irritating urine canses straining every few minutes, which is accompanied by intense pain. The kilneys are, after a time, infected, and become the seat of abscesses. Abscesses and fistulæ form around the base of the bladder, and the patient dies from exhaustion induced by the incessant pain and the septic fever.

\section{DIAGNOSIS.}

Tuberculosis should always be suspected when a young man of tubercular heredity develops a cystitis without any apparent exciting or predisposing cause, which runs a very persistent and intractable course, and is not cured by the ordinary treatment.

The cystoscope should be used with extreme precautions to avoid 
(at1-ing an infection of the bladder with jus-organisms. It may show tuturete in groups or dirseminated, or ragged. punched-out, irregular uluets.

I micmecupic examination of the pus from the bladder confim the diagnusis by discheing tubercle bacilli. There may be difficulty in finding the bacilli, eren after centrifugating, as they are with sanny. If none are fount. the sediment may be injected into the subcutancous tissues orer the abdomen of a guinea-pig. If the animal is lifled four weks later. and the lrmph-glands are tubercular or tubereular nodules are diseminated throughout the body, the diagnosis is established.

A careful search slould always be made for tubercular foci in neighboring orrans, and nodules may usually be found in the prostate, seminal resicles, or epididymis.

Tubercular incolvement of the hilney often exists and is overlooked. The symptoms are not marked: there is an absence of renal pain and colie and no tenderness on pressure. It may be suspected, howerer. when the urine is persistently of a low specific grarity and acid in reaction, and when the pyoria is intermittent in character, and the amount of pus is greater than could be furnished by the ulcers in the hadter.

If the erstoscope shows a tuberenlar ulceration about the month of one ureter, it is an evidence that the kidney on that side is affected; but the point can only be definitely determined by catheterizing the ureters or making use of llarrison's rectal segregator and examining scratatcly the wine from each kidney.

\section{PROGNOSIS.}

The prognosis in tuberculnsis of the bladler is always grave. and runcery is excendingly rare, but the disease may remain latent for yar- mules complicated by infection with progenic micro-organisms. This lonstons the juroces: the kinneys are lialle to become tubercular, and the blatiler grows rapidly worse.

\section{TREATMENT.}

The attemut has been made by Guyon. Reverdin, and others th cure vecical tuberculosis in its earliest stages by opening the bladder - uprapulic and curetting nut the nleer.

The recort to surgical measures in the beginning of tuberculosis 
of the bladder, in the hope of curing the disertor, is to-day practically abandoned. The bladder infection is almost always secondary to thbercular deposits in other organs which are out of reach, and sureical interference in the majority of cases only increases the rapridity of the course of the disease.

In incipient tubereulosis of the bladder the indication for treatment consists in improving the vitality of the tissues, and to this end life in the open air or a long sea-royage is the best measure.

Under this régime patients often improve and a few recover. After suppurative cystitis and pyelitis have set in, no benefit is to be derived from leaving home.

Creasote and.carbonate of guaiacol are recommended by some authorities, and by others are considered of no arail.

Local treatment is harmful, as a rule. The tubercular deposits camnot be reached, and there is danger, in bladrler-washing and instillations, of introducing pus-organisms and causing suppurative cystitis. After this has occurred, the instillation of corrosive sullimate (Guyon), using 20 drops of 1 to 5000 sohution and increasing up to 1 to 1000 , are often of marked benefit. The reaction following is rather severe, and may last five to six days, and should be allowed to subside before repetition. After the reactive symptoms have pased away, the pain of urination is lessened and the intervals between the acts are prolonged.

Nitrate-of-silver instillations are irritating, and aggravate the inflammation. This is so notably the case that a violent reaction following the use of nitrate of silver in a case of cystitis suggests that it is tubercular in origin.

Later in the disease, when the pain and frequency of urination become unbearable, morphia is the only means of relieving the sutiering.

As time goes by and opium fails to control the tenesmus and frequent micturition, permanent drainage of the bladder through a fistula should be established. This is not surcestul in relieving the difficulty entirely, but is the last refuge for the relief of the intruse suffering. The suprapubic opening is to be preferren, as the apparatus for receiving the urine can be more easily applied and the fatient is not long confined to bed, but can be up and ahout.

At the time of operation the bladder may be inspected, and if a few isolated tmerenlar nfeers are found they an be remored with the eurette or Paquelin cautery and jodoform rubbed into the spots. 
The subseyuent healing of the ulcers has a farorable influence upon the pain and tenesmus, and the permanent fistula in the bladder allords an opportunity for the complete and painless evacuation of ammoniacal urine and pus. and also admits of its cavity being kept clean by irrigations, and the congested mucous membrane can also be nedicated by instillations of corrosive sublimate or nitrate of silver.

When the bladder cavity is very much contracted and its walls are thick and rigid, suprapubic cystotomy cannot be so readily performed. and permanent drainage, through a fistula in the perineum has to be resorted to.

There is always the danger, in establishing an artificial fistula, that the round itself may be infected and become the seat of a tubercular infiltration, increasing the extent and rapidity of the progress of the disease. The perineal wound is much more liable to infection than the suprapubic opening, and should only be used when the suprapulbic fistula is impracticable.

\section{BACTERIURIA.}

This condition is characterized by the quantities of bacteria which are found growing in the urine, which is entirely free from pus. The mucous membrane of the bladder is perfectly healthy and the infection is confined to the urine alone.

On inspection the urine appears turbid and of a whitish-yellow color, and the odor is rery disagreeable, resembling stale fish or freculent material.

On centrifugating, and examining the sediment microscopically, it is found to be composed of the bacillus coli communis in enormous yuantities. Pus and epithelial cells exist in very small numbers.

It is often impossible to trace the manner in which the bacteria gain access to the bladiler, although in most of the cases reported there has been a condition of hrmaturia, enlargement of the prostate requiring catheterization, stricture, or recent gonorrhœa, and in women a recent infammation of the pelvic organs. It is therefore supposert that the lacteria enter the bladder $(a)$ through the urethra, (li) with the urine from the kidneys. (c) from the general blood-current, or (d) from adjacent organs through the lymphatics, although their place of proliferation is not discoverable. 


\section{SYMPTOMS.}

The symptoms of bacteriuria are not marked, and the tumbin urine with a foul odor is often the only sign.

Sometimes the urination is frequent and urgent, and accompanicul by a burning pain in the urethra, and rarely a chill followed by fever, which resembles urinary ferer in its onset and course, is noted.

\section{COURSE.}

The course of the disease is variable, occasionally brief and transient, sometimes more protracted, with remissions alternating with exacerbations, and it often becomes chronic, lasting for years.

\section{PROGNOSIS.}

The prognosis is rather unfavorable as regards a cure, unless the place of bacterial growth is accessible. If this is not the case, the bacteria are apt to remain permanently in the urine, but the general health does not suffer and the urinary organs remain in a healthy state.

\section{DIAGNOSIS AND TREATMENT.}

The diagnosis can only be made by excluding cystitis and finding the bacteria in the urine with the microscope.

The treatment consists in first removing any possible source of growth for the bacteria by relieving habitual constipation or enteritis. If the breeding-place of the micro-organisms is in some local condition, such as a posterior urethritis or stricture, it should be remorent.

When no cause is discoverable, the administration of urinary antiseptics internally,-salol, methyl-blue, and Urotropin,-in order (1) destroy the bacteria, is called for. The patient should drink frem of pure distilled or spring- water in order to mechanically wash out and remove the fermenting contents of the bladder.

Bladder-washing with solution of nitrate of silver or sublimate is, in general, of little use, and still at tinues it may be of some value. 


\section{TUMORS OF THE BLADDER.}

The new growths which necur in the bladder may be either benign or malignant. The benign tumors, or papillomata, appear in ditlerent forms: (a) As villous polypi, composed of loops of bloodresscls, which grow and project into the cavity of the bladder in long, waring strings, and (b) fibro-papillomata, which are pedunculated tumors of a firmer consistence.

\section{Malignant Tumors.}

Primary cancer of the bladder is exceedingly rare, but secondary deposits are not infrequent. The forms in which it presents are as

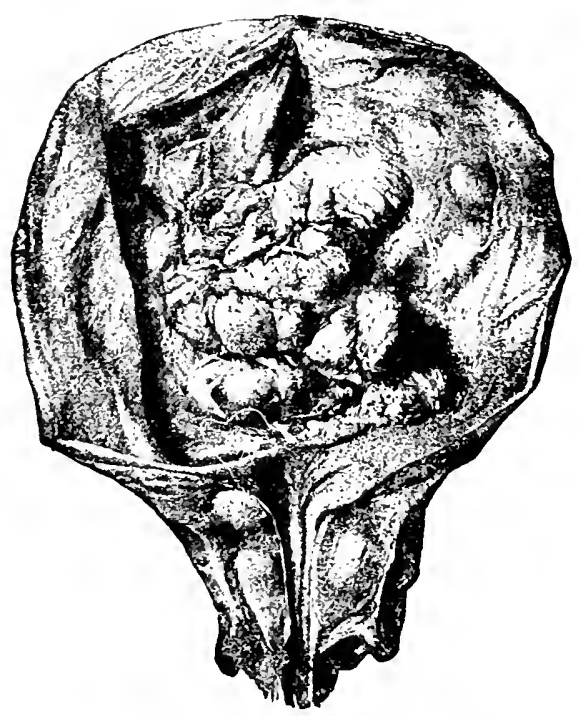

Fig. 49.-Carcinoma of the Bladder.

sarenula or carcinoma, which is either epithelial or the glandular(rlled rariety.

They are less apt to be polypoid in appearance, but are spread nut. infiltrating the tissues. The surface is covered with granulations $n$ villosities. and in the later stages is nlcerated and presents gaping nluers. The tumors are often multiple and their development is slow. 
Both benign and malignant forms of growth are usually located in the region of the trigone or near the openings of the ureters.

Tumors of the bladder predispose to cystitis, which in time leads to disease of the kidney, either suppurative pyelitis or hydronephrosis, or death may occur from the constantly recurring hæmorrhages.

If cancer of the bladder is primary, it is not apt to affect distant organs.

Vesical ealeulus is a frequent complication of new growth in the bladder from a deposit of the urinary salts upon the ulcerated bladder-wall or upon a piece of necrotic tissue separated by slonghing.

\section{SYMPTOMS.}

Hæmaturia is the most prominent symptom of tumor of the bladder. It comes on suddenly and without provocation, and may last a few hours or for some weeks. The quantity of blood lost is variable and fluctuating. At one time it may be abundant and upon the next urination the water may be almost elear.

Frequency of urination and tenesmus are often present in the later stages, and depend upon the cystitis, or are due to infiltration of the trigone and vesical neck by a malignant growth. Pain is never a prominent symptom, and when it occurs it is occasioned by the cystitis.

\section{DIAGNOSIS.}

The presence of a tumor due to malignant disease may often be felt by palpation, placing one finger in the rectum and making counter-pressure over the pubes with the other hand.

Any thickening of the trigone can be appreciated by introducing a sound into the bladder and feeling the thickness of the tissue which lies between the sound and the finger in the rectum.

Polypoid growths are not capable of being made out by palpation.

The cystoscope is not always available, on account of the amount and rapidity of the hæmorrhage obscuring the medium; but when it is possible to use it a definite diagnosis of the conditions can be marte by an experienced observer.

Cystotomy, either perineal or suprapubic, with digital exploration or inspection of the bladder, affords the means of making a positive diagnosis.

The suprapubic opening is to be preferred, because if a tmmor 
is present it can be removed more readily than through a perineal wound.

PROGNOSIS.

Benign tumors, if let alone, may cause death either from the repeated hrmorrhages or from pyelonephritis following cystitis. 'They can be readily removed by operation, but are apt to grow again.

Malignant tumors are difficult to remove completely, as they infiltrate the bladder-wall and are liable to recur. Authorities give the duration of life at from two to three years after the commencement of the disease.

\section{TREATMENT.}

The hæmorrhage is sometimes controlled by hot injections of alum, $3 \mathrm{iv}$ to the pint; fluid extract of hydrastis, $5 \mathrm{ij}$ to the pint; or acetanilid, 3ij to the pint.

Clots retained in the bladder, if moderate in size, may be let alone, as they will soften and be passed naturally.

If the bladder should become greatly distended by retention of urine, the clots may be withdrawn by a large catheter and suction syringe or a litholapaxy evacuating tube and aspirator.

If the bleeding continues and is persistent, suprapubic cystotomy should be performed without further delay. The suprapubic opening aftords an opportunity for inspecting the interior of the bladder, and through it the tumor may be removed.

Polypoid growths are best treated by cutting through the mucous membrane at their bascs, remoring the whole tumor, and sewing up the ineision witl catgut sutures, or they may be removed by crushing or twisting off with forceps or by the sharp spoon, or they may be burned off with the Paquelin cautery or the galvano-caustic loop.

Infiltrating growths, which are always malignant, cannot be remored by these means, but require the complete extirpation of the portion of the bladder-wall upon which they are located.

If the tumor should be found too extensive for removal, the suprapuhic opening should be maintained as a means of permanently draining the bladder of decomposing urine and blood-clots. 


\section{CHAPTER XII.}

\section{VESICAL CALCULUS.}

THE stones which form in the bladder are classified, according to their composition, into three varieties:-

(a) Uratic calculi, which are made up of uric acid and urates. They form about three-fifths of the total number of calculi, and are the softest of any in their consistence.

(b) 0xalic calculi are composed of oxalate of lime. They occur less frequently than the others, the estimated proportion being about 3 per cent. They are the hardest and heaviest of all the varieties, and are usnally studded with numerous projecting nodules, from which they derive the name of mulberry calculi.

(c) Phosphatic calculi are formed from phosphates and carbonates, which are often combined with urate of ammonia. Phosphatic stones are not so hard as the oxalic, but harder than the uratic stones. They are never due to constitutional or diathetic conditions, but are always the result of cystitis and decomposing urine, from which the salts are deposited and agglutinated together by the pus.

Stones composed of a single element alone are rarely met with. As a rule, two or more elements are found together, arranged in concentric layers around the nucleus.

The formation of a calculus is not a simple process of a deposit of salts, and it is a common occurrence for urine to contain crystals of uric acid, oxalates, or phosphates for a long time without the formation of a stone; but in the presence of albuminoid material these crystals change their molecular form, and gain a tendency to coalesce and adhere to each other and also to a sort of frame-work composed of colloid material, which is furnished by the pus.

The practical deduction to be drawn from this fact is that, while crystals may be present in the mine for years without the formation of a stone, a cystitis producing pus gives the necessary stimulns to coalescence, and a stone is very liable to form.

\section{NUMBER.}

Stones are usually single, although very frequently they are multiple; sometimes five or six may be present. and instances are nn recorl where three or four hundred stones were taken from the hialdiur. 
It occasionally happens that a single stone becomes multiple throun a process of spontaneous fracture.

It has been found in laboratory experiments that, if a calculus formed in a solution of gum were placed in a solution of a different epecific gravity, it would split up into segments. Hence it is probable that the instances reported of the spontaneous fracture of stones in the bladder, which have been accredited to different mineral or springwaters, is due to the effect produced upon the stone by surrounding it with urine whose specific gravity and reaction have been changed from their original state at the time of formation of the stone by the ingestion of large quantities of water. The albuminoid material forming the frame-work of the stone absorbs fluid and swells, bursting apart the laminæ of the stone and so fracturing it into segments.

\section{ETIOLOGY.}

For a calculus to form, it is absolutely essential that there should be a foreign body in the bladder, since the crystals must have a nuctous around which they may coalesce and adhere.

The nucleus may be a foreign body introduced from without, such as a broken catheter- or pipe-stem, pin, etc., or it may be a small piece of necrotic tissue which has been sloughed off from the bladder-wall.

In many cases of stone, howerer, the nucleus is composed of uratic crystals. These become agglomerated in the kidney and pass through the ureter, causing renal colic. They drop into the bladder, and, if they are not roided through the urethra, form a nucleus.

Predisposing Causes of Uratic and 0xalic Calculi. - As these stones are dependent upon a constitutional or diathetic state, certain things which influence the general condition of bodily health play an imprortant rôle in their formation.

It has been generally supposed that the habitual drinking of water inpregnated with the salts of lime was rery apt to occasion r.aic alculus. Investigations to determine this point show that the af calculus are not equally distributed through the limestone districts, and that they are just as common in the adjacent regions where the water is free from lime, and on these accounts the limutrme thenry has heen abandoned.

Diet and constitutional habit, however, are important factors in the production of stone, since the excretion of uric and oxalic acids 


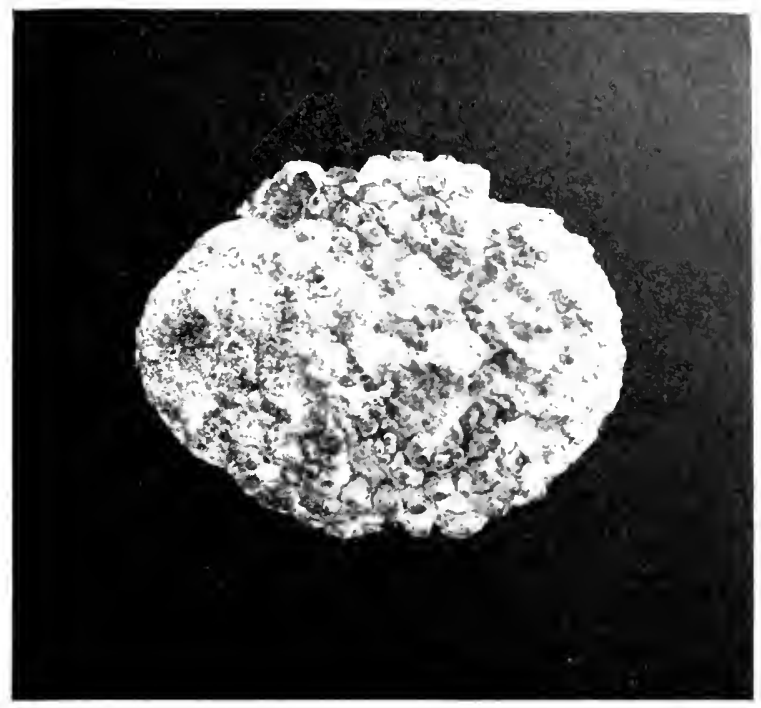

Fig. 50.-Oxalie or Mulherry Calmlus. (Author's Specimen, from Kings County Hospital.)

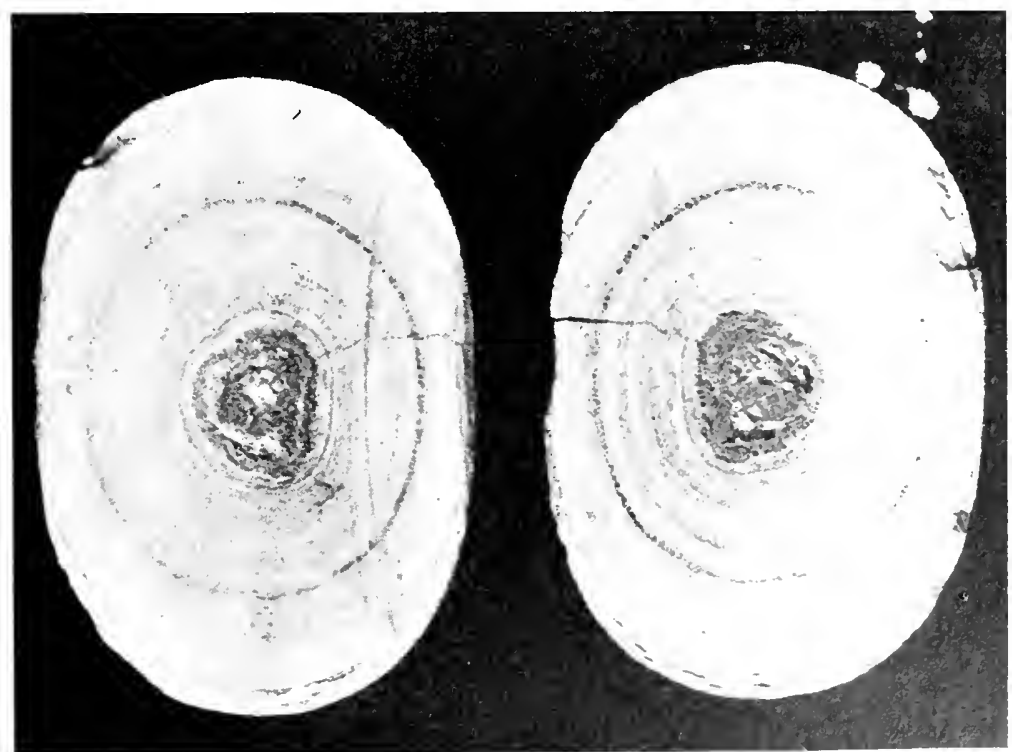

Fig. 51.- Tesical Caleulus, split in two Halves, showing molle of formation, by a deposit of I'ho-phates in comeentrie Lilyers, arenull al Uric-Acid Nueleus. Weight, 1370 Grans. (Authors speesmen. Frm Kings County Iospital.) 



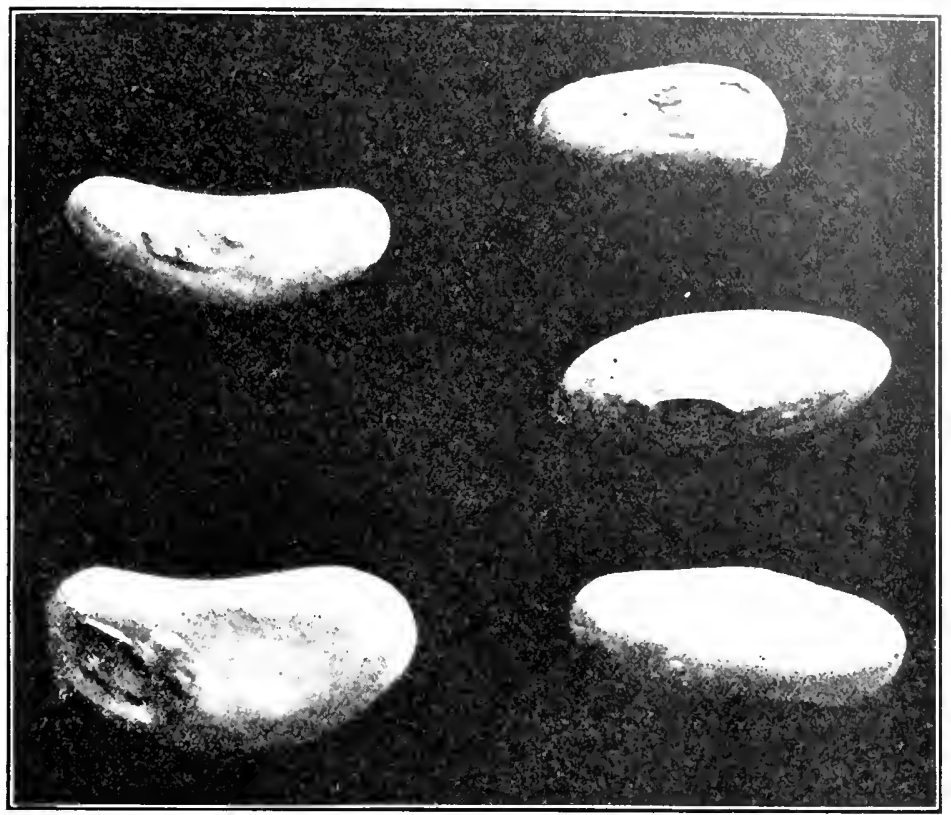

Fig. 52.-Multiple Phosphatic Calculi, removed by Suprapulic Cystotomy from same Patient. (Author's sperimen, from Kings County Hospital.)

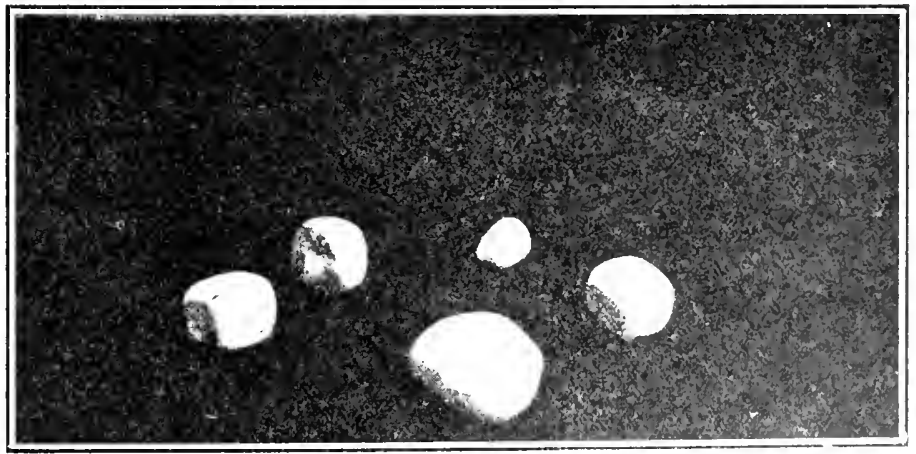

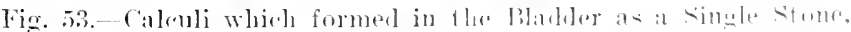

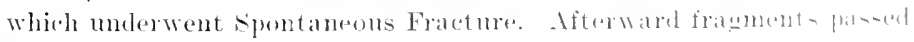
into the Prostatic Crethra, where they lewalne inlatede and wate

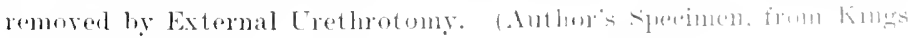
(ounty Ho-pital.) 

depends upon the quality of the food and the diathesis. On this account children with feeble digestive powers, who eat largely of nitrogenous food, are unable to perform the processes of oxidation completely, and the results of the retrograde metamorphosis of the tissues are eliminated, not as urea, which is freely soluble, but as urates or oxalates.

The same is also true of adults, who cat more nitrogenous food than they require, use alcohol and malt liquors freely, and take but little exercise. Such persons are subject to attacks of gout or various manifestations of lithæmia, which are included under the term of gouty diathesis.

The gouty diathesis is notably an inherited condition, and on that account different members of successive generations of a family are liable to develop oxalic or uratic stone in the bladder or some other gonty manifestation.

Phosphatic stones are not constitutional in their origin, but are dependent upon purely local causes. They result in consequence of urine decomposing and throwing down crystals of the triple phosphates, which are glued together by muco-pus, and form nuclei, arom which crystallization goes on rapidly.

On this account any obstruction which prevents the bladder from emptying itself-such as stricture, hypertrophied prostate, and paralysis - allows residnal urine to accumulate, and, if cystitis occurs, alkaline fermentation of the urine, deposit of crystals, and stoneformation result.

\section{SYMPTOMS.}

While it is possible for a small stone to exist for years without producing marked symptoms, and particularly so if it lie in the ponch behind an enlarged prostate, it is usual for a stone in the bladder to give rise to the following prominent symptoms: $(a)$ pain, (b) increased frequency of urination. $(c)$ blood, and $(d)$ sudilen stoppage of urine in full stream.

The pain is referred to the head of the penis under the lans, and shoots into the perineum and down the thighs.

It is felt especially at the end of urination, for, as the last few drops of urine are expelled, the muscular contractions of the biuliter drive the stone forcibly into its neck, causing a contusion.

The pain is increased by riding in a jolting wagon, by going downstairs, or even by walking. 
Increased frequency of urination oceurs through the day, when the patient is about on his feet and the stone is moving in the bladter. At night, when the individual is quiet in bed, the stone does not move, and the intervals between urination are longer.

Blood never appears in any large quantity in the urine, but a few drops are squeezed out at the end of urination, or it may be mixed with urine, giving it a smoky color. It is not a constant symptom, but intermittent.

Sudden stoppage of the urine in full stream is a very characteristic symptom, when it is present, and is caused by the stone being carried into the vesical outlet, obstructing it and shutting off the flow of urine like a valve.

It is rarely observed in old men with enlarged prostates, as the stone lies in the deep posterior prostatic pouch.

Cystitis always exists when a stone is present, and the symptoms of calculus are complicated by those of inflammation of the bladder.

\section{DIAGNOSIS.}

The elinical history of stone is merely suggestive of the condition, but to make a positive diagnosis the stone must be touched with an instrument or through an incision in the bladder or seen with the cystoscope.

Examination with Thompson's Searcher, or Sounding for Stone. - The patient should lie on his back, and with old men it is desirable

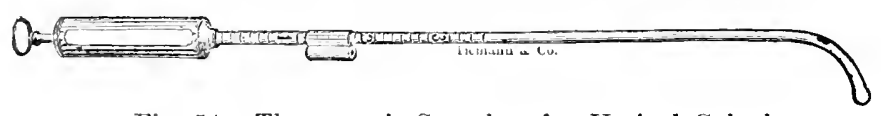

Fig. 54.-Thompson's Searcher for Vesical Calculus.

to elevate the hips so that the stone may roll out of the postprostatic ponch. The bladder shonld contain from four to six ounces of sterilized water, in order to distend its folds and allow the beak of the seareher to move freely.

The searcher is introduced after the manner of a sound, and the trigone and post-prostatic pouch should be examined by turning the beak of the seareher from side to side and rotating it behind the prostate, as the stone always lies in the most dependent portion of the bladder.

If the stone is not touched, the fluid in the bladder should be 
allowed to flow out through the hollow shaft of the searcher, and, as the bladder collapses, the stone is often brought up against the point of the searcher and the impact can be felt.

There are certain precautions to be observed in sounding for stone. The patient's genitals should be cleansed and the instruments must, of course, be sterile. Children should always be examined under chloroform, as otherwise they would be restless, and the bladder might be wounded by a sudden movement.

In old men there is a great deal of danger of minary fever. They should be examined at home and kept in bed from twenty-four

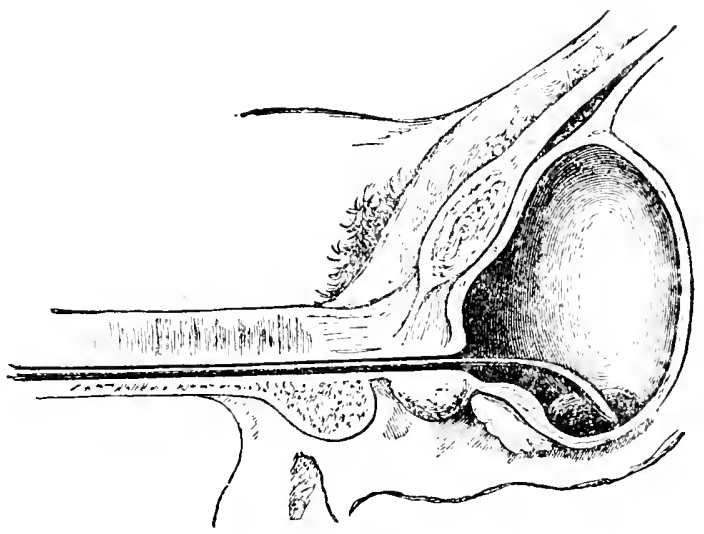

Fig. 55.--Searching for Stone Lying in the Post-prostatic Pouch.

to forty-eight hours after the examination. It is always desirable to administer salol or Urotropin for two days before searching.

If there is a strong suspicion of the presence of a stone, it is advisable to make all the preparations for immediate operation, in order to avoid the reaction which so often follows an examination of the bladder.

The presence of a stone is perceived by the searcher and gives rise to a sharp click, which can be felt and heard. Soft stones give a dull or nuffled sound when tonched, but the sound is sharper in character when a hard stone is struck.

The size of a stone may be measured by graping it between the jaws of a lithotrite; but the determination of the si:e or number of 
the stones is difficult with the searcher, and for this purpose the cystoscope is of great value.

With this instrument it is possible to see the stones distinctly, unless the bladker is bleeding freely, and their size, shape, and location can all be accurately ascertained.

Phosphatic stones are white and round, uratic stones are yellowish and oval, and oxalic concretions are dark and covered with bosses and sharp points; but it should be remembered that most stones are composed of different elements arranged in concentric layers. At the same time the position of the stone may be ascertained, for a stone lying in a deep pouch behind an enlarged prostate or attached to the bladder-twall as an incrustation or lying in a saccular dilatation will often be out of reach of a scarcher, and may be overlooked.

An examination of the urine often throws light on the character of the stone by revealing the predominant form of the urinary erystals.

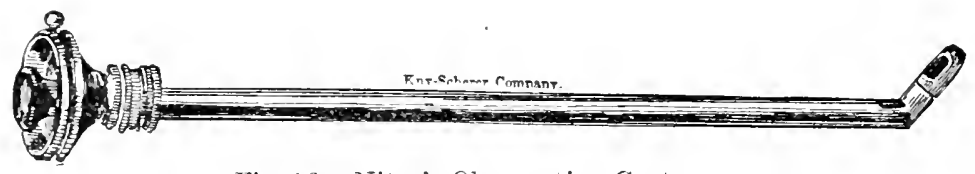

Fig. 56.-Nitze's Observation Crstoscope.

The litholapaxy pump is sometimes useful in detecting a small stone which elndes the searcher. The tube is introduced and water forced into the bladder with the bulb. The outflow of the water forees the stone against the orifice of the tube, and the click is appreciable to the ear and to the touch.

Since the introduction into practice of the cystoscope the exploration of the bladder through a suprapubic or perineal wound is rarcly called for.

In former times it was often necessary in doubtful cases, particularly in the instance of a calculus lying in a saccular dilatation or in the presence of an incrustation of the bladder-wall with urinary crystils.

\section{PREVENTIVE TREATMENT.}

The presence of erystals in freshly-voided urine which is still warm should be regarded as an indication that calculus is liable to form, and the tendency to the formation of uratic and oxalic stones 
should be guarded against by attention to the patient's general health. He should be cautioned against using an excess of nutrogenous food, sugar, or fat. It is in general thought best not to cut off all the nitrogenous food, but to allow a general diet, with a ruduction of the accustomed yuantity of meat.

Moderate and systematic daily exercise in the open air is of great assistance in faroring oxidation.

The ingestion of large quantities of pure spring- or distiller water has the effect of diluting concentrated urine, rendering soluble its contained salts, and flushing out the kidneys.

If the urine is strongly acid, citrate of potash is indicated in order to render it neutral.

If phosphates are abundant and due to imperfect assimilation, the mineral acids, particularly phosphorie, in doses of $m x v$ three times a day, and the vegetable bitters improve the digestive powers. The phosphaturia often disappears for a time and sometimes permanently under the use of Urotropin, gr. viij three times a day, or benzoic acid.

The adoption of local measures for preventing the formation of phosphatic calculi is much oftener crowned with success.

As these stones are always caused by the decomposition of alkaline urine in the presence of cystitis, the indications are $(a)$ to drain the bladder by removing a stricture, if present, and evacuating residual urine, in cases of enlarged prostate or atony and distension of the bladder in paralytics, and (b) to cure the existing cystitis by bladder-washing.

In this way the decomposition of urine is checked and the precipitation of phosphates and carbonates ceases.

Attempts to dissolve stones after their formation have been made for many years, but, while some have been moderately successful in the laboratory, no method has yet been found which is capable of dissolving a stone in the bladder.

The various waters from mineral springs which have derived some reputation as solvents owe it to the fact, already spoken of, that in a few extremely rare instances spontanenus fracture of the stone occurs when the specifie gravity of the fluid surrounding it is altered. 


\section{OPERATIVE TREATMENT.}

Litholapaxy, or crushing the stone and immediately washing out the fragments from the bladder, is the operation of choice in all cases of vesical calculus.

It is a prerequisite of the operation that: I. The lithotrite and eracuating catheters should be introduced easily and without wounding the urethra or prostate. In children under sixteen years of age litholapaxy is regarded by many surgeons as inadmissible on account of the small size of the bladder and urethra.

It is adrised, however, by others whenever the urethra is large enough to admit the instruments.

II. The stone must be movable in the bladder, of moderate size, and not too hard.

If a stone lies in a saccular dilatation or is adherent to the bladder-wall, it camnot be crushed. Very large stones form such a great quantity of detris after crushing that the operation of washing out becomes too protracted.

The contra-indications to litholapaxy are (a) extreme prostatic hypertrophy. The prostate may be so much enlarged that the lithotrite cannot be introduced, or, even if passed into the bladder, it cannot reach and grasp the stone as it lies in the post-prostatic pouch.

(b) Tight stricture of the deep urethra. Such a narrowing of the urethra will prevent the passage of the instruments, and requires external urethrotomy for its cure, and, at the same time, the stone may be removed through the perineal wound, although if very large it may have to be crushed with a lithotrite first.

(c) Severe cystitis which does not yield to ordinary treatment. In this condition it is better to remore the stone through an incision, which will subsequently serve for the prolonged drainage of the bladder and at the same time afford an opportunity for its irrigation.

(d) Contracted and irritable bladder does not admit of sufficient distension, and is too intolerant of instruments to allow the stone to be crushed.

(e) Nephritis and suppurative pyelitis, since uræmic coma and death follow litholapaxy, when the kidneys are diseased, more frequently than after the eutting operations.

Technique.-The patient, with his rectum previously emptied, is anæsthetized, lyimg upon his back. The hips are elerated, to allow the stone to roll out of the post-prostatic ponch. 
The bladder is washed out and filled with six to cinth ounces of boric-acid solution.

The lithotrite is passed through the urethra after the manner of a somd, and rests on the floor of the bladder behind the prostate,

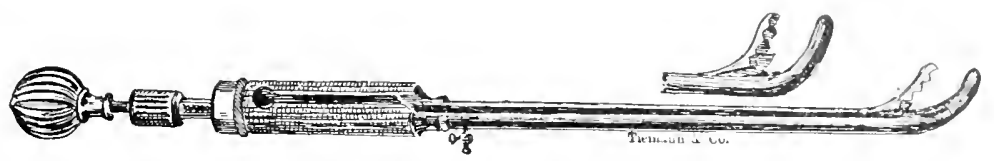

Fig. 57.-Bigelow's Lithotrite.

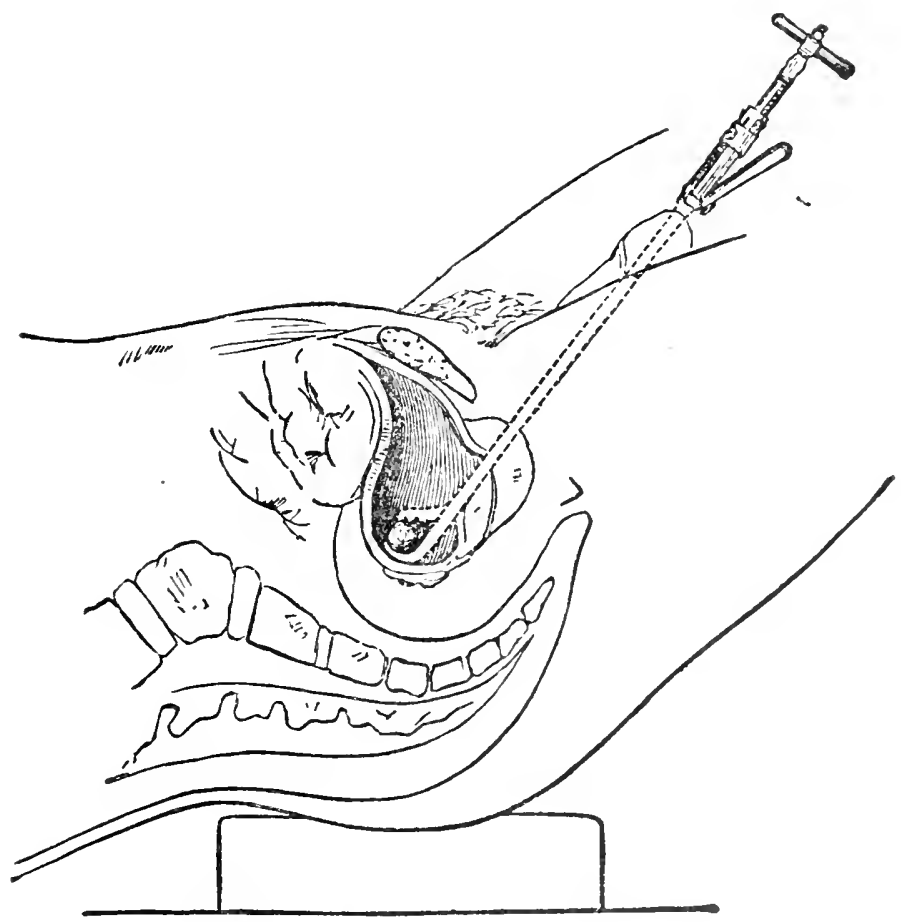

Fig. 58.--Method of Grasping the Stone in Lithotrity.

with its beak pointing upward. The lithotrite, from its weight, sinks in and forms a depression in the wall of the bladder. Ordinarily the stone lies alongside the beak of the lithotrite, and when the jaws are opened it rolls in between them. The jaws are closed and the 
stone is felt to be grasped. If the jaws fail to seize the stone, the hades are opened in the upright position, turned over on one side, and shut along the floor of the bladder. If the stone is not found, the manceure is repeated in the opposite direction.

If the stone lies in a deep post-prostatic pouch, raising the patient's hips may roll it out within reach, or it may be pried up by a finger in the rectum.

In the event of these failing, the jaws of the lithotrite may be turned so as to point downward, in the hope of seizing the stone as

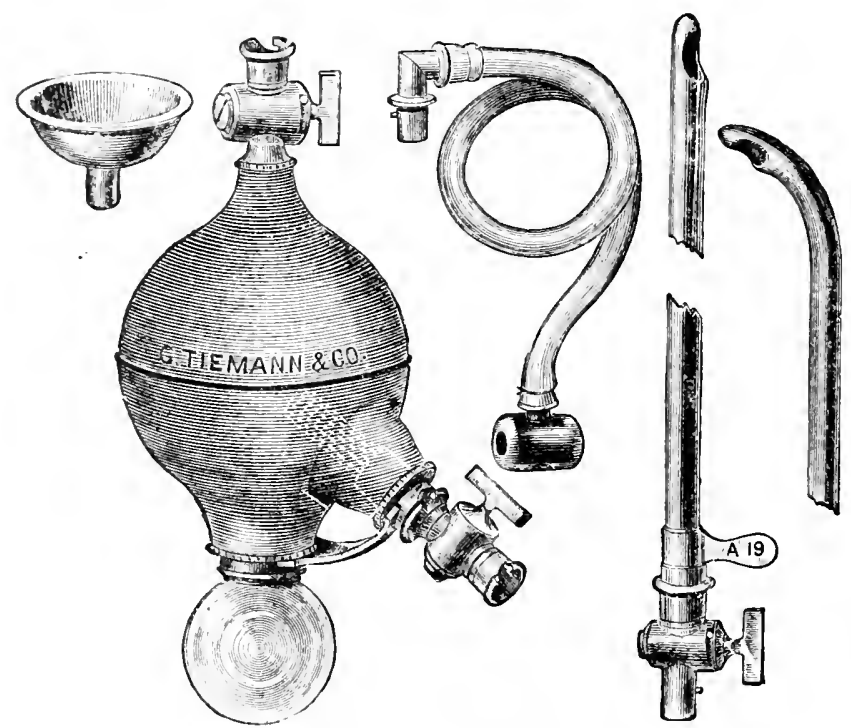

Fig. 59.-Bigelow's Evacuator.

it lies; but in this case there is always danger of nipping and cutting out a piece of the bladder-wall.

When the stone has been caught between the jaws, the resistance it offers is easily felt on approximating them. The blades of the lithotrite are then locked, and by turning the handle the jaws are screrred together, comminuting the stone into fragments. The manourres of opening the jaws, catching the stone, and breaking it are repeated till the surgeon perceives that no large fragments are left, and the lithotrite is withdrawn.

The next step is the evacuation of the débris. The evacuating 
tube is introduced into the bladder and its contained fluid allowerl to flow out with a gush, carrying with it some of the fragments. The bladder is then filled with water by pumping in the contents of the bulb, and the fluid then flows out again into the receiver, carrying more fragments. The pumping is continued till no more fragnents come away, and auscultation over the bladder fails to perceive the click of a fragment remaining behind which is too large to enter the tube. If this is the case the lithotrite should be introduced again and the piece crushed and pumped ont.

The after-treatment consists in keeping the patient in bed and allowing him to drink freely of water, to keep the kidneys active.

If retention of urine occurs, it is well to tie a eatheter in, in order to aroid the irritation of its frequent passage.

After ten days have passed the bladder should be pmmped onee more to remove the sand and mucus which is left and which might serve-as a nucleus for another stone, and the entire absence of fragments should be verified by a cystoscopic examination.

Mortality of Litholapaxy.-The following statistics, compiled by Cabot, show the death-rate at different times of life:-

Children under 14,241 cases, with 4 deaths $=1.66$ per cent. $\begin{array}{lllllllll}\text { Adults, } 14 \text { to } 50, & 400 & \text { " } & & 13 & * & =3.25 & \text { " } & \text { " } \\ \text { Old men, } & 433 & \text { " } & \text { " } & 26 & \text { " } & =6 & \text { " }\end{array}$

Perineal Lithotomy.-Until within a few years, and before the technique of litholapaxy and suprapubic lithotomy was perfected, the only way of removing a stone was through a perineal incision.

At the present time the perineal operation has fallen into almost entire disuse, except in children. It offers the advantage of good drainage for the bladder, but its disadvantages are hæmorrhage, which is difficult to control, and the bruising and laceration of the tissues caused by dragging the stone through a small ineision, which leads to infection and slonghing. For these reasons the mortality is higher with stones over $1 \frac{1}{4}$ inches in diameter after the perineal operation than following suprapubic lithotomy.

Perineal lithotomy is particularly dangerous in old men with enlarged prostates, and should not be undertaken withont some urgent reason.

It is also difficult to reach a stone from the perineum when the prostate is very much enlarged, on account of the increased length of the perineal distance. Before the fifty-fifth year a small stone can 
be removed with greater safety through a median perineal incision than by suprapubic lithotomy, but ordinarily such cases are better treated by litholapaxy.

The indications for median perineal lithotomy may be tabulated as follows:-

(a) Small stones of not more than $1 \frac{1}{4}$ inches in diameter, complicated by stricture of the urethra, which demands external urethrotomy.

(b) Small stones in the presence of atony of the bladder with

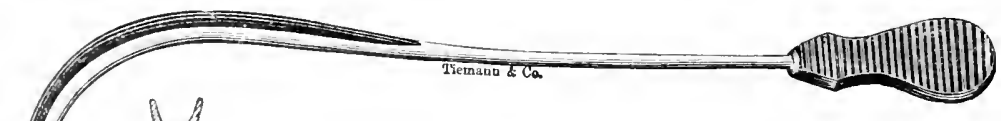

Fig. 60.-Lithotomy-staff.

no expulsive power, and chronic cystitis, provided that the prostate is not very much enlarged.

(c) Contracted and irritable bladder, which does not admit of sufficient distension for suprapubic cystotomy and is too intolerant to allow litholapaxy.

Perineal Lithotrity.-Reginald Harrison has enlarged the scope

Fig. 61.-Lithotomy+knife.

of median lithotomy by devising a lithotrite which is introduced through a median incision and by which stones of considerable size can be crushed in the bladder and removed through the perineal wound.

I'erineal Lithotomy.-Technique.-(a) Median Operation.-This is simply a boutonnière, or external urethrotomy, and is performed by introducing a grooved lithotomy-staff through the urethra into the bladder.

The staff is held steadily by an assistant and the surgeon makes 
a direet thrust or stab with a long, straight bistoury, an inch and a half in front of the rectum, through the tissues lying in front of the urethra, and strikes the groove in the staff. A gorget is then slid along the groove until it enters the bladder, which is explored

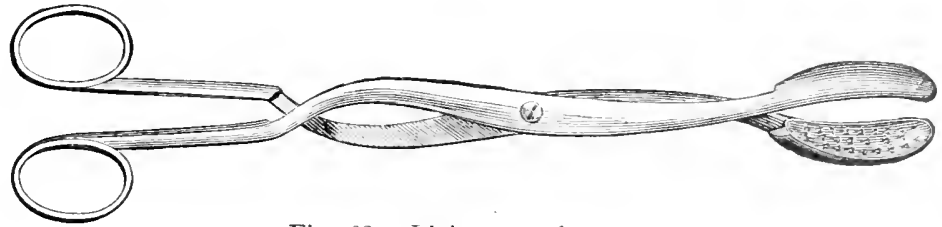

Fig. 62.-Lithotomy-forceps.

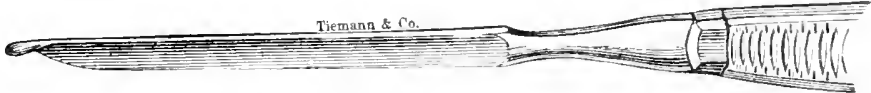

Fig. 63.-Blizard's Probe-Pointed Knife.

by means of the finger. If a stone is found it is grasped by the stone forceps and withdrawn, if not too large.

If the stome prove too large to pass through the wound, (b)

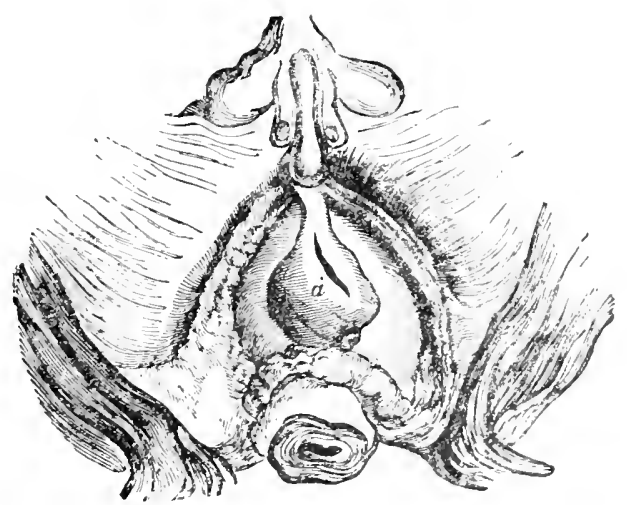

Fig. 64.-Incision Through the Urethra and Prostate in Lateral Lithotomy.

perineal lithotrity may be performed by introducing Harrison's lithotrite, crushing the stone, and either extracting or pumping out the fragments with a litholapaxy evacuator.

The operation may, if desired, be converted into (c) luteral 
perineal lithotomy, which was the operation formerly used exclusively in all cases of stone. A Blizard linife is slid along the groove in the statr, as it rests in the bladder, until the point of the knife has entered the bladder. The knife is then withdrawn, making a sweeping cut, outward, downward, and a little to the left, enlarging the original median incision and cutting through the left lobe of the prostate, the perineal muscles, and the skin.

Fig. 65.-Lithotomy-scoop.

In this way the incision is made of sufficient size to permit the extraction of large stones with the forceps or scoop; but, on account of the drawbacks of hæmorrhage and laceration of the tissues, this form of lithotomy is rarely used at the present time.

After-treatment of Perineal Lithotomy. - The wound is never sewed, but is left to heal by granulation. Hæmorrhage is inconsiderable after the median operation, and is easily controlled by a gauze packing around a large soft-rubber catheter. It is often very severe after a lateral lithotomy, and often requires the use of the shirted canmula to hold it in check.

The mortality of perineal lithotomy has been tabulated by Freyer and Rosenthal, as follows:-

Deaths before the 20 th year. ... 5 to 8 per cent.
" in adults $\ldots \ldots \ldots \ldots \ldots \ldots$
" above the 40 th year.....

These figures refer, in the main, to the operation of lateral lithotomy, and apply less to median lithotomy or lithotrity.

Suprapubic Lithotomy. - Suprapubic lithotomy was first performed in the year 1550 by Pierre Franco, but did not gain favor until 1880 , when the application of aseptic methods and improvements in the technique of the operation reduced the previously high rate of mortality.

At the present time, in the cases where litholapaxy cannot be applied and a cutting operation must be employed, suprapubic lithotomy is, under most conditions, the operation of choice. 
The advantages of suprapubic over perineal lithotomy are:--

(a) The suprapubic operation admits of complete inspection and evacuation of the bladder.

(b) Wounding the ejaculatory duets, neck of the bladker, and rectum, and dangerous hæmorrhage from incised blood-ressels are avoikled.

(c) The laceration and bruising of the tissues, which occurs when a large stone is dragged through a perineal wound, are aroided.

(d) The bladder is rendered easy of access, while in the presence of a considerable degree of prostatic hypertrophy the perineal distance is so much increased that it may be impossible to reach the bladder from below.

The special indications for suprapubic lithotomy hare been tabulated as follows:-

(a) Tery large stones, even though they be soft. (b) Large, hard stones. (c) All fixed stones, incrustations of the bladder-wall, sacculated stones, etc. (d) Cases where a high degree of prostatic hypertrophy exists which prevents the seizure of the stone by the lithotrite.

The technique of the operation is based upon the anatomical fact that, when the bladder is filled, the fold of peritonemm, which lies in front of it, is raised up two inches above the pubes, leaving a space, called the space of Retzius or prevesical space. uncorered by peritoneum, through which the bladder-wall may be safely ineised.

Technique.-The India-rubber bag which was used in the earlier operations for distending the rectum, and so bringing the bladder nearer to the abdominal wall, is rarely, if ever, employed at present. The Trendelenburg posture is only exceptionally required.

A catheter is introduced through the urethra and the bladiler is filled with from eight to twelve ounces of sterilized water by means of a syringe. Helferich and Bristow use air for this purpose.

The catheter is withdrawn and a rubber band is tied around the root of the penis.

An incision is made in the median line of the abdomen. beginning three inches above the pubes and extending down over it. which divides the skin and subentaneous fat. There is no linea alla in this region and the incision is carried directly through the muscular bundles, and the transversalis fascia is divided. The edges of the wound are held apart by large angular retractors and the bladter appears lying at the bottom of the wound. It is recoginizud by its 
shape and by the prevesical fat, which is adherent to it. If any doubt exists, it may be punctured with an exploring hypodermic syringe.

In old men the peritoneal fold in front of the bladder is sometimes unusually long, and extends down low into the space of Retzius. In such a case it should be peeled up from the bladder and held out of the way. If the peritoneum is accidentally wounded, it should be at once sewed up with fine catgut.

After the bladder is exposed, it should be steadied with a sharp hook. thrust through its wall at the upper angle of the wound, and two long silk retraction sutures are passed through the bladder-walls, at the sides of the wound.

These are held by an assistant and the bladder is opened between them by means of a stab-like thrust, with a long, sharp bistoury. As the water in the bladder is flowing ont, the incision may be enlarged to the desired extent by means of a straight, blunt-pointed bistoury.

The cavity of the bladder is then searched with the finger, and the stone removed with a lithotomy-scoop or forceps. If it is desired to inspect the interior of the bladder, its walls may be distended by Watson's bladder speculum and the cavity illuminated by Pilcher's electric lamp or a head-mirror and reflected light.

The advisability of sewing the wound in the bladder or of leaving it open is still nuder discussion. All authorities, however, agree that in the presence of severe suppurative cystitis and foul purulent urine. the bladder should not be sewed, but left open for drainage. With a moderately-healthy bladder in young persons, opinions differ, and the following plans are adopted:-

(a) Kocher sutures the wound in the bladder with a double row of sutures, the first row including the mucous membrane, and the second row, which may be a continuous suture, includes the muscular and serous coats of the bladder. The space of Retzius is packed with ganze, and the wound in the abdomen left open for several days. If the stitches in the bladder hold, the abdominal wound may be closed by secondary suture. Drainage of the bladder is provided for by a permanent catheter introduced either through the urethra or preferably through a small perineal incision.

(b) In addition to suturing the wound in the bladder the abdominal incision is also cloced by sutures, excepting the lower angle, through which a wisp of gauze is inserted for drainage. 
(c) The wounds in the bladder and abdominal wal! os: rint - 21 tured, but are left open and allowed to heal by smanulation.

By this latter method the convalescence is prolonged and the patient is subjected to a great deal of inconvenience from the urine flowing out over the wound and wetting the bed continually. To aroid this objection, the following plans for drainage have been snggested:-

(a) Guyon's donble tubes. Two large rubber drainage-tubes are introduced through the abdominal wound into the base of the bladder, and gauze packed around them.

(b) In addition to the suprapubic tubes, permanent catheterization throngh a boutonniere is carried out.

(c) The Dawbarn bladder-drain consists in a reservoir of water hung upon the wall and from which the water flows out through in rubber tube. This rubber tube is connected to a single tube introduced into the bladder loy a T-shaped glass tube. As the water flows out of the reservoir a suction is ereated which keeps the bladder empty.

(d) The Bloodgood bladder-drain which is a rubber bag attached to a hard-rubber tube entering the bladder. The urine flows out through it and is collected and retained in the rubber bag.

The length of time required for the healing of the wound is from two to three weeks when the bladder is sutured, and from three to six weeks when the wound is left to heal by granulation.

The after-treatment may be attended with difficulties, and there is always danger of urinary infiltration of the walls of the wound. In order to avoid this it has been recommended, and particularly so in the presence of a foul cystitis, to open the abrlomen first. and to make the incision into the bladder several days later.

In the time elapsing between the two operations gramlations have had time to form and septic alsorption is prevented.

The mortality statistics of suprapubic lithotomy collecter by Cabot show the following results:-

AGE. CASES.

Adults, 14 to 50 vears. 100

old men .......... 53

$\begin{array}{cc}\text { Deaths } & \text { Percestage. } \\ 12 & 12 \\ 1 \% & 32\end{array}$




\section{OPERATIONS FOR CALCULUS IN CHILDREN.}

Perineal Lithotomy.-The median operation is impracticable in children, on account of the difficulty in withdrawing a stone through the small posterior urethra. For the same reason Harrison's lithotrite cannot be used. The lateral operation, however, can be readily performed with much greater safety than in adults, for, as the prostate and urethra grow larger, they become more vascular, and the danger of hemorrhage increases.

'The lateral operation has a drawback in the danger of wounding the ejaculatory seminal duct and producing sterility on that side, in after-life. The mortality is about 3 per cent., and lateral lithotomy is still generally considered to be the operation of choice in the case of small stones in children.

Litholapaxy in children is attended with some difficulties, on account of the small size of the urethra interfering with the introduction of the lithotrite and the relative hardness of the stones, as met with. In spite of this it has been performed a great many times, and the death-rate is low: only about 3 per cent. As far as the death-rate is concerned, there is but little choice between lateral lithotomy and litholapaxy.

Suprapubic cystotomy in children is more dangerous, according to the collected statistics, than either of the other operations, the mortality being about 10 per cent., although stones one and onequarter inches in diameter or orer are considered too large for lateral lithotomy and must be removed through a suprapubic incision.

The size of a stone is readily estimated in children by the bimanual cxamination with one finger in the rectum and the other hand over the bladder. 


\section{DISEASES OF THE PROSTATE.}

\section{CHAPTER XIII.}

\section{SENILE HYPERTROPHY OF THE PROSTATE.}

THE prostate is placed like a sphincter around the first inch of the urethra. It consists of two lateral lobes and a median central portion connecting them, which is sometimes spoken of as the third, or median, lobe. In its histological structure it resembles the uterus in the female, and is composed of muscular fibres, glandular elements, and a connective-tissue stroma uniting them.

While the gland may be enlarged as a result of gonorihuea or sexual abuses in young men, true hypertrophy of the prostate never occurs until the decline of life, when other degenerative changes incident to advancing years begin. It is almost never observed until the patient is past his fifticth year.

Sir Henry Thompson states that hypertrophy of the prostate exists in 34 per cent. of men at and above 60 years of age. and that it produces manifest symptoms in abont 15 or 16 per cent. of the cases, when it is enlarged.

The cause of hypertrophy of the prostate is entirely unknown.

\section{PATHOLOGY.}

The pathological change consists in a general enlargement of the entire organ, or the increase in size may be confined to one or both lateral lobes or to the median lobe. The character of the enlargement of the gland and its size and consistency depend upon which of the normal tissues of the prostate have been chiefly affecteri by the process of hypertrophy.

The first change which oceurs is a growth of the gland-tubules with their associated muscle. so as to form a new glami-sulictance closely resembling the normal prostatic substance. This constitutes the first or glandular stage of hypertrophy.

In this stage small tumors often form in the sulstanes of the 
prostate, causing an enlargement, which is irregular and which may impinge upon the mrethra and cause it to become distorted.

After a rarying length of time degenerative changes set in, which ultimately convert the new tissue into a mass of more or less dense fibrous connective tissue, containing the atrophied remains of the glandular and muscular elements. This constitutes the second or fibrous stage.

After the adenomatous structures have been obliterated by the degenerative changes, the enlargement of the prostate becomes even and symmetrical, and instead of being soft and elastic, as in the glandular stage, it is very hard and dense.

The hypertrophy may consist in an overgrowth of both glandular and stromal tissues in equal proportions, causing a symmetrical enlargement of the prostate, which is not excessive and seldom causes any symptoms.

The size to which the prostate may grow depends upon the nature of the tissue involved. The prostate may be only slightly increased in size or it may become as large as a hen's egg or an orange, and in very exceptional instances may reach the size of a cocoa-nut, and fill up the entire pelvis.

Form of 0bstruction.--In certain cases the lateral lobes may be enlarged, but in such a way as not to interfere with the urinary outlet, and it is possible to empty the bladder entirely. As a rule, however. the posterior median portion, or third lobe, becomes enlarged, and assumes the shape of a bar or dam across the mouth of the bladder, behind which the urine accumulates and cannot flow out, or the outgrowth may be more circumscribed in form and act as a ball-valve, which shuts down over the resical orifice.

Alexander has recently suggested that the enlarged prostate interferes with the rhythmical contraction of the detrusor muscle, in the region of the trigone, and the bladder, on this account, is incapable of emptying itself of the last few ounces of urine, which are normally expelled by the action of this muscle. This view will serve to account for the cases in which there is no appreciable obstruction in the shape of a projecting outgrowth from the prostate, but where residnal urine accumulates.

In many instances the enlargement of the prostate does not produce any symptoms, and it is only of consequence when it acts as an obstruction placed at the outlet of the bladder, thus preventing it fiom completely evacuating its contained urine. 


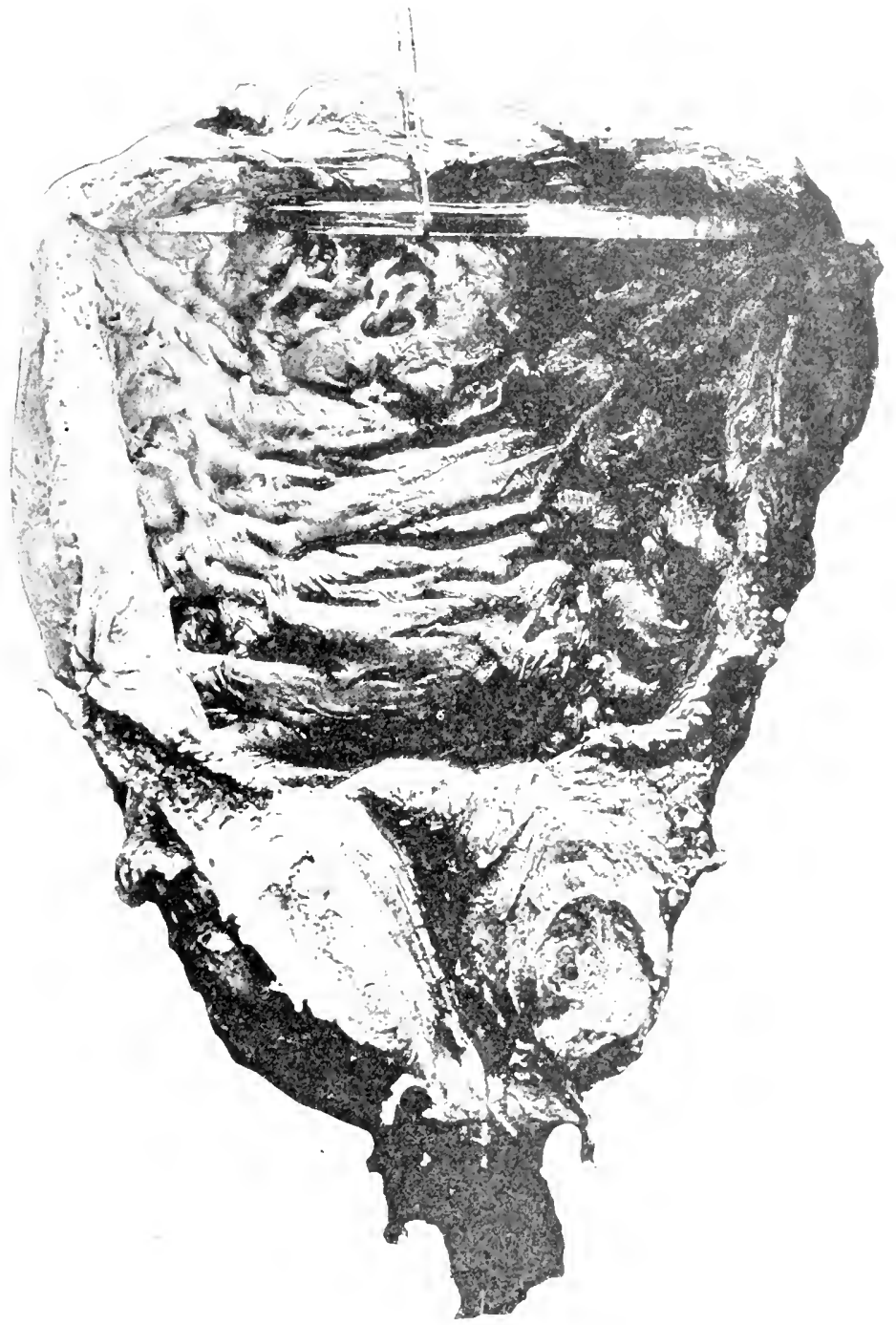

Fig. 66.-Prostatic Hypertrophy. Median Enlarcement. in the form of a Bar. Suitable for Bottini's Operation. A litrge Blatder. (By Courtesy of Dr. F. S. Watson.) 



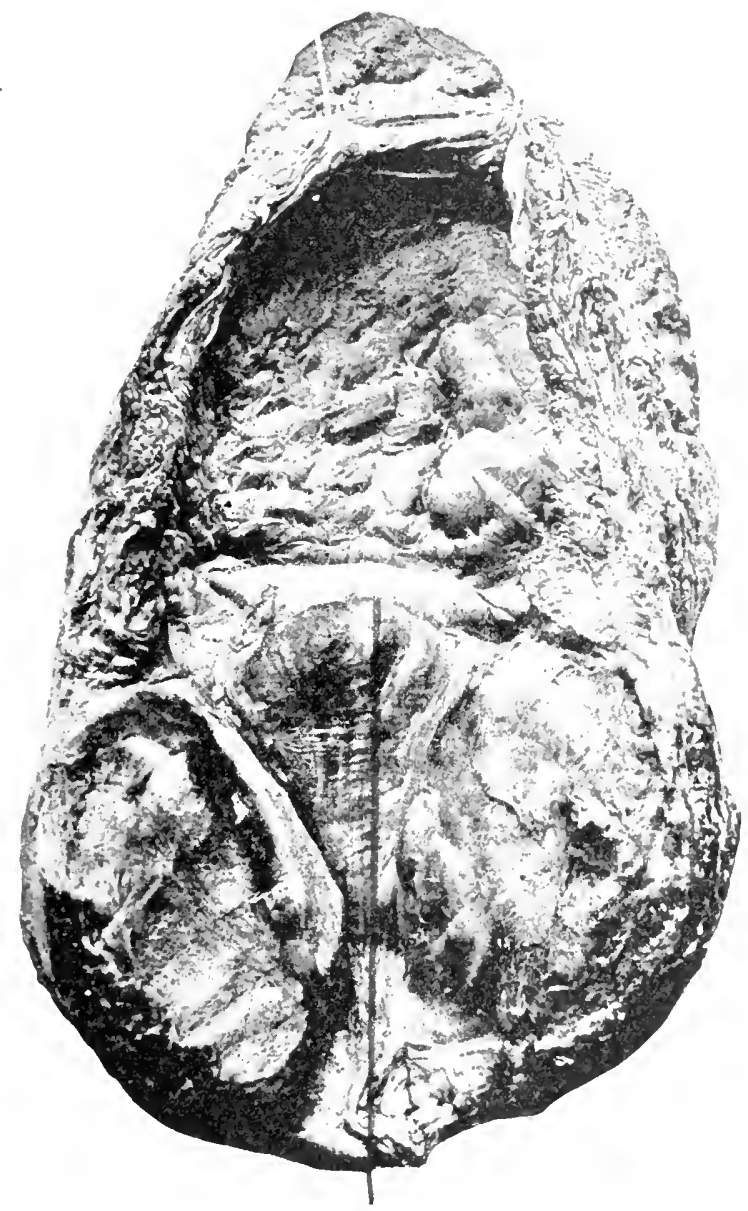

Fig. 67.-Prostatic Hypertrophy. Enlargement of Lateral Lobes and Median Portion. Bladder Contracted and Non-distensible. (By Courtesy of Dr. F. S. Watson.) 



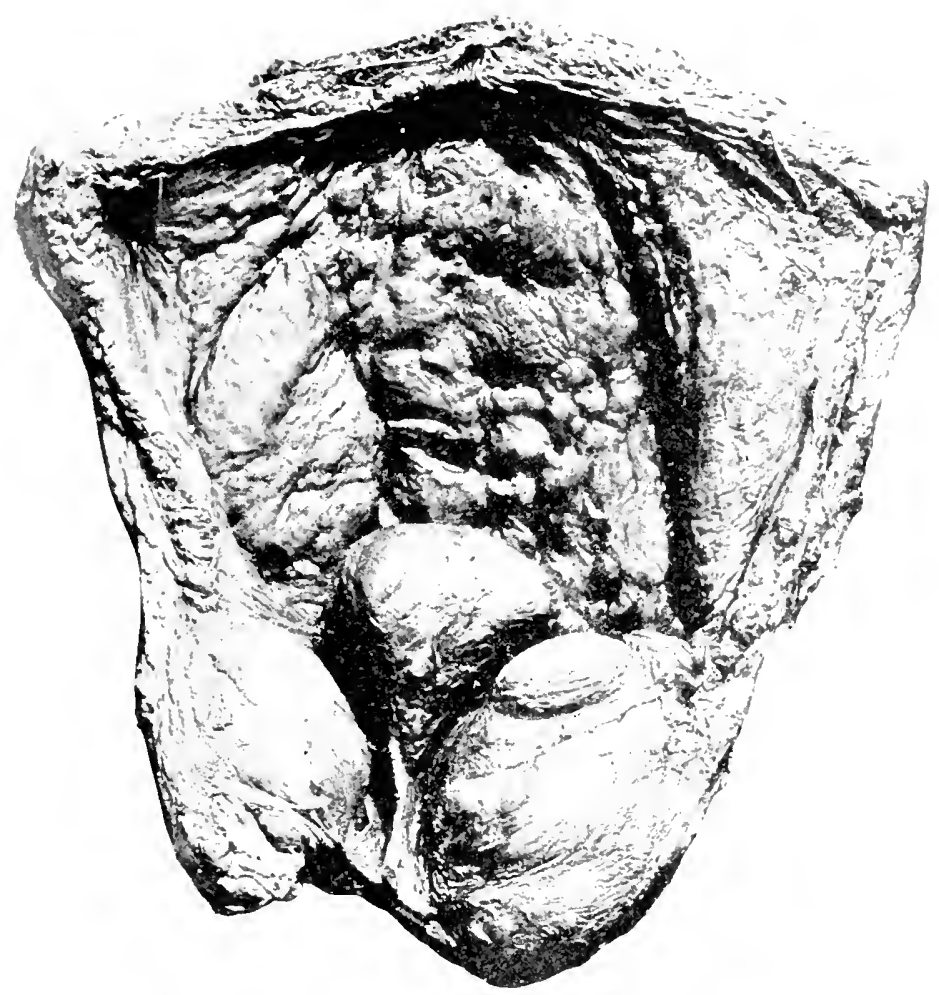

Fig. 68.-Prostatic Hypertrophy. Enlargement of Lateral and Median Lobes. Deep Post-prustatic Puuch. (By Cuurtesy of I)r. F. S. Watson.) 

The various symptoms and diseased conditions which ofenr in consequence of the prostatic hypertrophy are all due to two (n) mlitions:-

(a) The obstruction caused by the enlargement of the prostate at the resical outlet interferes with the minary outlow, and the bladder cannot be completely emptied by the muscular etlorts of the patient. Residual urine accumulates, pathological changes ncenr in the bladder-wall, and secondarily the neter and kidneys become aflected.

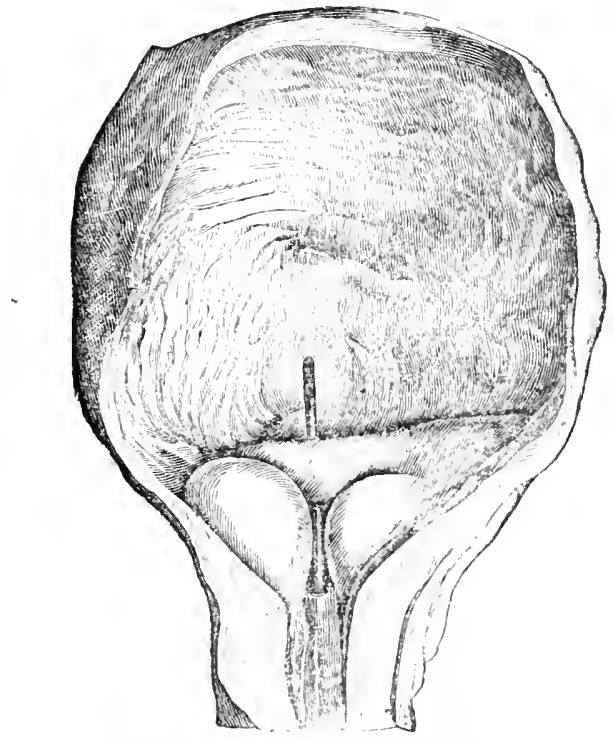

Fig. 69.-Prostatic Hypertrophy. Enlarement of the Lateral Lobes, with Increase in Size of the Nedian Purtion, Forming a Lar, Through Which a False Passage has been Made.

(b) The return-flow of venous blood from the bladeler-wall is prevented by the pressure exerted upon the reins by the enlitred prostate, and a congestion of the bladter-ralle occurs as a result.

Changes in the Urethra.-In consequence of the entargement of the prostate, notable alterations orcur in the prostatic uretha:-

(a) It becomes elongated.

(b) The normal curve is changed.

(c) The calibre of the urethra, instead of being ronnt. is thattened from side to side by the pressure of the lateral lobes, so that it becomes a vertical slit. 
As a result of these changes catheters of ordinary shape are often inapplicable to cases of prostatic hypertrophy, and special catheters have to be employed.

\section{SYMPTOMS.}

One of the first symptoms of enlarged prostate which a patient observes is:-

I. Difficulty in starting the flow of urinc.

In addition to this is noted:-

II. Deficiency in the force of the stream.

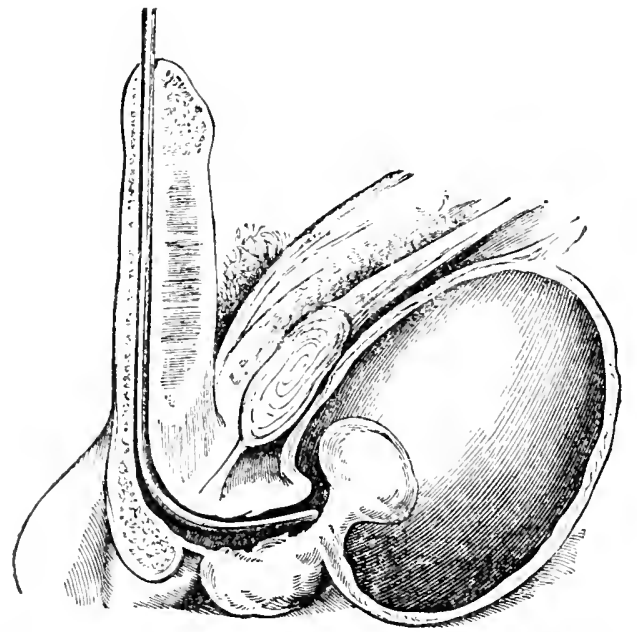

Fig. 70.-Prostatic Hypertrophy. Pedunculated Middle Lobe Obstructing Passage of a Catheter.

Both of these symptoms are occasioned, in part, by the feeble and atonic condition of the muscular fibres of the bladder, and partly on account of the prostatic obstruction.

III. Frequent calls to urinate especially at night.

This symptom is explained by the fact that the enlarged prostate presses on the plexus of veins lying around it, and so obstructs the flow of renous blood. As the blood from the bladder is returned through this plexus, any interference with its circulation causes a passive congestion of the bladder-walls. Through the day the muscular activity improves the circulation, but at night the muscles are in repose, and a passive hyperemia occurs. 
The increased frequency of urination at night oftun helys to ristinguish a case of enlarged prostate from one of resical calculus. In the case of a stone the desire to urinate is more frequent during the day, when the stone is rolling about in the bladder, wut at night the patient is quiet in bed, and the stone does not canse much irritation.

One of the most important conditions from the stand-point of treatment and proguosis, which enlarged prostate gives rise to. is residual urine. Residual urine may be defined as the urine which remains in the bladder after the patient has voluntarily tried to evacuate it completely, and always occurs as a result of an obstruction.

Residual urine exists in the majority of cases of prostatic hypertrophy, and in the early stages and in cases of slight obstruction may only amount to an ounce or two. The tendency is for it to increase in quantity as the bladder-walls become atonic and the prostatic enlargement becomes more dereloped; so that in exceptional cases it may reach a quart in quantity.

After residual urine has existed for a varying length of time cystitis is generally excited by the entrance into the bladder of microorganisms, coming from the urethra or rectmm or introduced artificially upon a dirty catheter.

The causation of cystitis is favored by the stagnant resilual urine and also by the catarhal mucus, which is formed in the congested mucous membrane lining the bladder as a result of its condition of chronic passive hyperæmia.

The urea of the urine is decomposed by bacterial action. assisted by the presence of the catarrhal muens, and carbonate of anmonia is set free. The urine becomes alkaline in reaction and ammoniacal in odor, is strongly irritating, and adds still further to the existing inflammation of the bladder-wall.

When cystitis is well established, the frequency of urination is increased, and the patient urinales as frequently by day as at night.

The mucous membrane of the bladder surrounding the urethril orifice becomes turgid and congested, and serves to occhule still more the outhet from the bladder, and the residual mine increases. The various changes dependent upon cystitis and obstruction lead to changes in the wall of the bladder. In order to furnish sulficint propelling force to overcome the olstruction formed hy the protatic overgrowth, the muscular fibres of the bladter-mall undergu hypertrophy. The bundles of muscular filmes grow larger and purojet into the bladder, and diminish its capacity. 
The muscular eflorts of the bladder force the urine between the hundles of muscle, and in time saccular dilatations form, which may he as large as the bladder itself. As these sacs are only covered outside with peritonem and the serous wall of the bladder and have no muscular fibres, they cannot empty themselves. In consequence, the urine stagnates and decomposes, and it frequently happens that a calculus forms in them.

In course of time as the case progresses the hypertrophy gives place to atrophy and distension. The distension of the thinned bladder-wall may be very great; so much so, that it may contain a ynart of residual urine and reach as high as the umbilicus.

The distension oceurs so gradually that the patient is unaware of his condition, and notices only a slight feeling of weight in the abdomen, and that his habitually frequent urination is slightly increased. As the residual urine increases, the bladder grows more distunded and the intervals between the acts of urination become still slorter. After the bladder is stretched and distended to its utmost (apacity a new symptom occurs: that of incontinence of urine, or constant dribbling.

The bladder is so full that it can hold no more, and the overtaxed sphineter yields, allowing the eseape of a small quantity of urine every few minutes.

The conditions of decomposing residual urine and cystitis just described provide snitable conditions for the formation of phosphatic calculi. As Keyes expresses it, "Stone is the logical sequence of obstruction to urinary outflow aided by resical catarrh."

The stagnant decomposing urine deposits salts, which become glued together by muco-pus into a solid eoneretion, or are deposited mum a nuclens, which may be a bit of neerotie tissue sloughed off from the bladder-wall.

A single stone or several may exist without causing any symptmm. ant they may lie unsuspected for months in the poeket behind an mlareed midnle lobe. Their surfaces are smooth, and they are preventer? from rolling about in the bladder, and the weakened muscular filues of the bladder camnot drive them forcibly out of the mot-prositic ponch against the resical orifice during the art of unination.

It smmetimes haprens that after hypertrophy begins the bladderntwat of arowing weakener and atonic and ultimately becommalurges hypertrophy with contraction. In this condi- 
tion the overgrowth of the prostate does not form an obstruction to the outflow of urine, and residual urine does not accumulate, and the bladder, instead of being distended and enlarged, unctergoes a diminution in the size of its carity, so that it may only contain six or eight ounces of urine when fully distended.

In addition to the contraction of its size there is also a permanent congestion of the vesical neck from pressure on the reins by the enlarged prostate. This venous engorgement oceasions an extreme irritability of the bladder, with an almost constant desire to urinate.

Of the two conditions spoken of, the patient with an atonied, relaxed bladder which does not cause him trouble, even though it is incapable of emptying itself except by catheter, suffer's far less inconvenience than the man who has an hypertrophied irritable bladder, and is tormented continnally with an intolerable desire to urinate.

Changes in Kidneys and Ureters.-As the obstruction offered by the prostate increases, a greater amount of muscular eflort is required to empty the bladder, and, as a result of the pressure, the urine is forced back into, the ureters, causing them to become distended, and sac-like dilatations form at various points.

The backward pressure and damming back of the mine is continued, and saccular dilatations of the pelves of the kidneys occur, giving rise to hydronephrosis.

The salts of the stagnant decomposing urine are deposited, and calculi are apt to form in the various dilatations in the kidney.

The process of dilatation becomes further complicated by germinfection, which usually ascends up the ureters, and as a result cantes pyelitis or abscess of the kidney.

Retention of urine occurs in nearly every case of enlarged prostate, and presents itself in two forms:-

(a) Acute Retention.--This occurs suddenly in an individunl who up to that time had been able to pass water with a fair deyree of freedom. It is frequently brought on by chilling or wetting the surface of the body and taking cold. The sudden congestion of the mucous membrane around the vesical orifice canses it to swell ni] and occlude the opening entirely, in the same way that a cold in the head will close up the nasal passages.

On the other hand, the retention may be occasioned by a spatsmodic contraction of the cut-off muscle (spranodic stricture) which oceurs as a reflex from constipation and scylialia in the reetum, or an 
arit combition of the urine from overindulgence in wine or malt liguters.

(b) Chrnic retution, as already described, is caused by the obstrucion at the resical ontlet from the enlarged prostate and partly indumat by the atonic condition of the muscular walls of the bladuer.

\section{DIAGNOSIS.}

In the case of a man orer fifty years of age, complaining of difficulties of urination, enlarged prostate should always be suspected.

It is desirable to conduct the examination systematically, and to that end

I. A rectal examination should be made, to feel the prostate and jullue of its size and consistency and to determine, if possible, whether the enlargement is fibrous or glandular in character.

uf course, only the posterior portion of the gland can be felt throngh the rectum, and it is impossible to determine the shape of the obstrueting overgrowth in this way, but its density and the rxtent of the enlargenent of the entire gland can be determined by the rectal touch.

II. The quantity of residual urine should be collected and neasured after the following method: The patient is requested to pass water, and endeavors to empty the bladder completely. A catheter is then introduced into the bladder, and all the urine which remains behind and which the patient was unable to void spontaneously is known under the term "residual urine." The residnal urine should be measured and set aside for microseopic examination.

The quantity of residual urine indicates the extent to which the mostatic enlargement interferes with the complete emptying of the bladder: but in order to determine the shape of the outgrowth which projects up into the bladder and blocks its outlet, we must have recourse to the cystoscope.

It the time of collecting the residual urine we can also determine the lemath of the urethra by measuring the distance from the ye of the cathoter to the point upon its shaft to which it is necessary to introrlue it before the urine begins to flow. This measurement often fails to lemonstrite an existing enlargement, as a decided alnumbl of olstruction may be present which eauses but very little "humpintion of the urethra.

III. IV mus of a cystoscopic examination it is possible to see whenent of the mildle lobe projecting upward, and to some 
extent to see a lateral lobe if it impinges upon the resical outle As the eystoseope is introduced through the urethra it will also serve to exehde the presence of a tight stricture, and by its nse in the bladder we can prove the presence or absence of resical mhluhus, which is so apt to be present in cases of enlarged prostate with resichal urine.

There are certain precautions to observe in every examination of prostatic eases: The patients are old men whose ritality is low and who are more or less feeble. Their urinary pasages are in an irritable condition, and they are very liable to urinury ferer, and any prolonged or rough examination is apt to be followed by ehills, ferer, and constitutional disturbances. For these reasons the instruments should be aseptic, and when practicable it is desirable to cleanse the urethra and bladder by irrigation before instrumentation.

The instruments should be used with extreme gentleness to aroid any traumatism, which would allow the entrance of micro-organisms into the circulation, and the first examination should not be too prolonged.

It is desirable that the patient should be examined for the first time in his own home, so that he can go to bed at once after the examination, and thus avoid the necessity of going ont-of-doors, rumning the risk of chilling the body.

A distended bladder should never be emptied at the first sitting. Cases are on record where sudden death has oceurred when a full bladder has been emptied by the eatheter with the patient in a standing position.

Although such an event is, of course, rare, drawing off the entire contents of the bladder allows the enlarged blood-ressels which have been acenstomed to the support of a certain amount of fluid to collapse, a transudation of blood follows; so much so that a great deal of hæmorrhage may ocenr, and the existing cystitis is always made worse.

If the bladder is considerably distended, it is alwars better to leave one-half or one-fourth of its contents in it, or to inject six or eight ounces of warm salt or borax solution and leave it in.

\section{GENERAL TREATMENT.}

In all cases of prostatic hypertrophy hygienic measures alre very important in preventing attacks of retention of urine. T'The pratient should wear flannel underelothing in winter, and. as the circulation of 
blood is pon in the feet and they are the parts most easily chilled, woolen stockings should be worn.

The should aroid any overindulgence in alcohol or malt liquors, although a glass or two of claret or a little whisky may be permitted.

The bowels onght to receive due attention, and constipation should be guarded against. Any orerdistension of the bladder with retained urine has the effect of still further weakening the atonic muscular walls, and on this account the patient should be instructed to pass his water at regular intervals, two to four hours apart.

In the early stages of prostatic hypertrophy, when the enlargement is only moderate in size and not very dense, and when the quantity of resichal urine is small, the chief source of annoyance to the patient is from the irritability of the bladder, which causes a frequent desire to urinate.

Such cases are often benefited by the passage of a large-sized steel sound, which relieves the irritability of the bladder and overcomes the muscular spasm of the urethra. The action of the sound is to press ont and empty the engorged venous plexus around the prostate, and while it does not prevent the increase in size of the gland, it seems at least to retard its growth. 'The sound should be used once in every five days, and allowed to remain lying in the urethra from ten to fifteen minutes at a time.

The presence of residual urine offers an important indication for treatment.

If the residual urine is only one or two ounces in quantity and clear in color. it is only necessary to pass the catheter once in four to eight weeks, in order to observe the progress of the case and ascertain that the obstruction is not becoming greater or the atony of the badder-wall increasing.

In time, however, the residual urine increases in amount, and, when it reaches three or four ounces in quantity, the catheter should be employed once a day to remore it from the bladder, a convenient morking rule (if the urine is sterile) being to use the catheter once daily, preferably at bed-time, for three ounces, twice daily for six onnces, and then once more for every additional two ounces.' With sterile urine it is rarely necessary to catheterize oftener than once in four hours.

Tnfortunately, however, the bladder rarely escapes infection for any longth of time after beginning catheterization, and then a new element is introduced into the case: that of cystitis. Inflam- 
mation of the bladder is generally ushered in by some rise in temperature, which may run for a few days and subside or may continue for some length of time, as a result of absorption into the blood of septic material.

The frequency of the desire to urinate is notably increased, and the urine contains pus and in the early stages blood may be present.

The fermentative changes in the urine rapidly cause its decomposition, and it becomes strongly alkaline in reaction and ammoniacal in odor.

Aftcr cystitis has lasted for a short time the contractility of the muscular fibres of the bladder-walls is still more impaired, and the residual urine increases in quantity, so that the catheter has to be used more frequently, in order to prevent the accumulation of urime, which is stagnant and soon decomposes.

The indications for the treatment of this condition may be summed up as follows:-

(a) Drain the bladder of residual urine.

(b) Keep it as clean and aseptic as possible, and check decomposition of the urine by means of bladder-washing and the administration of urinary antiseptics by the mouth.

For the treatment of the cystitis the reader is referred to the chapter on the treatment of "Chronic Cystitis."

The ordinary routine treatment of a case of prostatic hypertrophy, consisting in bladder-wasling and daily catheterization, can be readily carried out by the patient himself, if he is moderatcly intelligent, after he has been instructed by his attending physician.

The best form of catheter for him to use is the soft-rubber instrument, and, indeed, every patient with enlarged prostate shonld be familiar with the method of passing the catheter and should keep one at hand, as he is always liable to an attack of retention of urine from some slight cause, and unless prompt relief can be obtained the bladder-wall may suffer irreparable damage from the distension and stretching caused by the accummlation of urine.

An important part of the instruction of the patient consists in teaching him the proper method for the care of the catheter. This is a very material point in the management of the case. and, if cystitis is not already established, the frequent use of the catheter generally induces it, unless the greatest care to aroid infection is taken by means of scrupulous cleanliness.

It is not difficult to cleanse the outsire of the calluntur, but the 
interior is its most dangerous part, on account of the difficulty of disinfecting it.

After using the catheter a stream of hot water should be allowed to mu through it from a fancet or forced through with a syringe, in andition to serubbing off the ontside with soap and water. But "wen this is not sufficient to remore all the accumulations of pus and urine. retc.

boiling the catheter in plain water is a perfect means of disinfeeting the soft-rubber instruments, and, by having a number of them, they can all be boiled at once and laid away in clean towels and a fresh one taken for each catheterization.

The formalin sterilizing cabinet also affords a reliable means of disinfecting, both the soft-rubber and the gum-elastic instruments, after first scrubbing and allowing water to flow through them, and the plan of sterilizing a number of instruments at the same time may be adopted.

These methods, howerer, camnot always be applied to every case, as in the instance of travelers, who cannot carry the necessary sterilizing outfit. In such cases the catheter may be kept sterile by immersing it in a bottle of carbolic-acid solntion from 2 to 5 per cent., after washing, and allowing it to remain there. ${ }^{1}$

In every case the catheter should be frequently inspected, and discarded at once when it becomes dry and cracked.

At the time the patient is instructed as to taking care of his catheter he shonld also be tanght to wash the glans penis with a cotton sponge and soap and water every time before the catheter is introdnced, in order to avoid carrying micro-organisms into the urethra from the head of the penis.

But in spite of all precantions it is most exceptional for the urine to remain clear, and, as a rule, a slight degree of cystitis is contimually present. On this account and to lessen the tendeney to the formation of calculus, it is very desirable that the patient should use irrigation of the bladder once or twice each day.

"'The following solution is now being used in Berlin for sterilizing gumelastic catheters:-

R Gilycerin,

Trater. of each $\tilde{\tilde{3}}$ viij.

Corrosive sulblimate.................... gr. viij.

After six hours' immersion laboratory experiments show the atheter to be sterile, and prolonged immersion in the fluid does not roughen or eraek the surtaces. 
This ean be very readily done, at the time he nuses the milnoter, by means of a rubber douche-bag or fomtain-syringe raind up three feet high and attached by its tube to the end of thr athetr. The mild solutions, either boric acid, salt solution, or furmanganate of potash are suitable for this purpose.

Commencement of Catheter-life.-In cases of prostatic hypertrophy where there is little or no cystitis and the quantity of residnal urine is only from four to six ounces, if due preeautions are taken to guard against cystitis, the patient rery soon becomes aceustomed to the use of the catheter. But, in those instances where the recidual urine amounts to ten ounces or more, a considerable amount of constitutional disturbance usually follows the first catheterization. Lrinary fever generally oceurs, and, if the kitneys were previously cliseased and the patient is very old and feeble, it is by no means rare for the case to terminate by death.

It is desirable, then, before beginning catheterization, in the presence of large quantitics of residual urine, to warn the patient that there is some risk attached to the procedure, and to insist that he shall remain quietly at his home and for the most part in bed for a fortnight. During that time the urine should be drawn by the eatheter three or four times a day and from four to six omnces of boric-acid solution thrown into the bladder each time that the urine is withdrawn, so that the bladder will not be left entirely empty.

Urinary antisepties should be given by the mouth, but it is better to ayoid washing out the bladder for the first week or so, nntil a certain degree of tolerance is established. Under these precantions the advanced cases of prostatic hypertrophy with an excess of residual urine are introduced into catheter-life with a minimum degree of risk. But even in spite of all care patients who are old and feeble, and are suffering from chronic Bright's disease or prelitis, sometimes develop a chronic form of urinary fever, induced by the beginning of catheter-life, which terminates fatally.

In these instances death would bare resulted from the kidney disease, in any case, after a short time, and, had the nst of the eatheter been commenced at an earlier period in the divase, the secondary involvement of the kidneys womld have becn aroited.

Retention of Urine--Wost old men with enlargerl prostates suffer at some time in their lives with an attack of acute retention of urine, from spasm of the ent-off muscle and swolling of the muens membrane at the vesical outlet. Such a comation calls for prompt 
cutheterization. The use of prolonged hot baths and a full dose of opinm, which so often relieves a spasmodic stricture in a young man, is not to be recommended in retention of urine from an enlarged prostate. The orerdistension of the bladder must be relieved at once, as the stretching of its muscular fibres induees a condition of atony.

'The form of calheter to be selected depends upon the shape which the orergrown prostate has assumed. It is often found impossible to introduce a flexible soft-rubber catheter, and it is necessary to try various shapes, until one is found which will override the obstruction offered by an enlarged middle lobe or an adenomatous tumor which deflects the urethra to one side.

The form of catheter which is most frequently serviceable is the Mercier catheter, with the end turned up (condé). The angled end usually slips up past an enlarged middle lobe and enters the bladder. The catheter bicoudé acts in a similar manner and is useful when the overgrowth of the middle lobe is excessive.

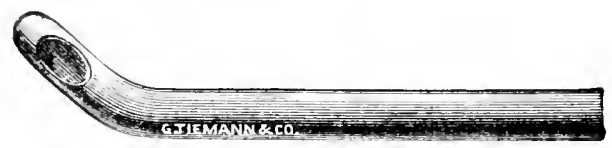

Fig. 71.-Mercier Catheter Condé.

The sitver prostutic catheter, with a long beak and an exaggerated curve, is especially designed to fit the elongated sweep of the urethra distorted by the prostatic overgrowth, and is frequently successful in reaching the bladder. When it fails to enter the bladder the following manipulation is often successful: The surgeon has provided himself beforehand with a common English eatheter with a stylet, which has been previously prepared by giving it an exaggerated curve, and it is then laid away until needed. When it is wanted, the stylet is withdram and the catheter is introduced into the urethra. The warmth of the body causes the curve to increase, and the point of the instrument orerrides the obstruction. The eatheter may also be introduced with the stylet in it down to the prostatic urethra. When it reaches this point the stylet is withdrawn and the point of the instrmuent curves around the enlarged prostate.

In the manipulations with the silver and English catheters due care should be used not to make a false passage in the swollen tissues arvund the prostate. The attempts to reach the bladder with a filiform 
guide and a tunneled sound threaded over it are of very little use exeept in cases of stricture.

A bladder which is full and distended, if it has bcen so for more than a few hours, should nerer be completely cmptied at one silling, but six or eight ounces of urine or boric-acid solution shonlu be left in it, to support the blood-vessels and give the muscles sonething to contract on.

If all attempts to enter the bladder are unavailing, in order to relieve the retention it is necessary to have recourse to suprapubic aspiration of the bladder.

The needle attached to the aspirator is thrust directly downward, a finger's breadth above the pubes, and penetrates the bladder withont wounding the peritoneum, as when the bladder is distended the fold of peritoneum covering it is raised up two and one-half inches above the pubes.

Aspiration affords prompt relief; but, while the bladder has been emptied for the space of a week by means of repeated aspirations, it cannot be done with safety more than a few times.

Suppuration often results after three or four tappings, or the bladder-wall becomes soft and leaks; so that aspiration can only be depended on as a temporary expedient, for a day or two.

In certain cases of enlarged prostate, which are suffering from chronic retention, it is desirable to keep the bladder empty and at the same time avoid the frequent introduction of a catheter; to attain this end continuous catheterization is accomplished by introdneing a soft-rubber eatheter into the bladder and tying it, so that it cannot be forced out.

The indications for the use of continuous catheterization are smmmarized by J. W. White as follows:-

(a) When great difficulty is experienced in introducing the catheter.

(b) When much bleeding follows the introduction of the catherer, as is generally the case when mnsuccessful attempts at catheterization have been made before.

(c) When much cystitis with pminlent or ammoniacal urine exists and the frequent passage of a catheter is impracticalule on acemint of the pain and hemorrhage which it pronuces.

By means of continuons atheterization the hlanter is irainer and readily washed out, and a general improvement in the symptoms takes place. 


\section{CHAPTER XIV.}

\section{OPERATIVE TREATMENT OF HYPERTROPHIED PROSTATE.}

'line removal of the obstruction oflered by an enlarged prostate, by nleans of a surgical operation, is not to be thought of in patients who have but a small quantity of residual mine and little or no cystitis. and when eatheterization is easy and not accompanied by pan or blecding. In many instances the patient can be made entirely comfortable throughout his life by regular catheterization.

In some cases, however, when the catheter has been used for a long time, the prostatic hypertrophy increases in size, the contractile pow of the bladder lessens, the eystitis grows worse, and the presence of a small quantity of urine in the bladder causes extreme tenesnuls until it is removed. As a result of the frequent demands for urination, the catheter has to be passed so often that the urethra becomes irritable and bleeds freely, and the eystitis grows progressively worse. In the expressive words of J. W. White the condition of the patient is that of "approaching break-down in catheter-life." These are all indications that eatheterization will soon have to be discontinned and some other means of draining the bladder adopted.

Indeed, all of the surgical operations for prostatic hypertrophy are directed to the one end of draining the bladder, either through the removal of the obstructing portion of the prostate or by means of an artificial permanent fistula.

A list of the operations may be tabulated as follows:-

Radical Operations.-

Bottini"s Operation.

Prostatotomy $\left\{\begin{array}{l}\text { Perineal. } \\ \text { Suprapubic. }\end{array}\right.$

Prostatectomy $\left\{\begin{array}{l}\text { Suprapubic, } \\ \text { Perineal, or } \\ \text { Combination of both. }\end{array}\right.$

Tasectomy.

Cistration, or Orchidectomy.

Palliative operations, in which no part of the prostate is removed, are mity madertaken for the purpose of draining the bladder through a fistulous opoung:- 
(a) Perineal cystotomy and drainage through a cathour petainent in the bladder.

(b) Suprapubic cystotomy.

(c) Puncture through the substance of the prostate with a trocar.

\section{BOTTINI'S OPERATION.}

Bottini's operation consists in buming channels through the enlarged prostate by means of a specially-devised instrument, heated with the electric current, and introduced into the bladder through the urethra. The instrument was devised by Bottini, of Pavia, Italy, and used by him for orer twenty years.

Description of Instrument. - The instrument is shaped like a lithotrite, and is provided with a thin platino-iridium blade, which is concealed when the instrument is closed, and is mored backward or

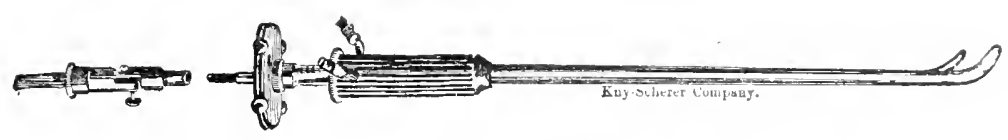

Fig. 72.-Bottini's Instrument, as Modified by Freudenberg.

forward in a slot in the shaft by turning a wheel at the end of the instrument. This blade is heated to a dull cherry red by means of an electric current conducted to it from a storage battery, through a cord attached to the extreme end of the instrmment. All parts of the instrument, except the blade, are prevented from becoming lieated by a stream of cold water, which constantly circulates through it.

Technique of Operalion.-General anasthesia is not required; a 4-per-cent. solution of cocaine is introduced into the posteriur urethra. after first washing the bladder with boric acid and letting it rnn ont again through the catheter. Bottini's instrument is introduced into the bladder and hooked up against the projection of the midlle lutu. The cold-water stream is turned on, and when ererthing is in rumliness the electric current is switched on, a pause of ten seconds is mante for the blade to become hot, and then the wheel is slowly tumml, causing the red-hot blade to slide out from its place of contealmont and slowly burn its way through the tissues of the prostate. Alter 
the incision has been made deep enough the wheel is reversed, and the blade is slid hack into its place of concealment, charring for a eccoml time the tissues of the incision, and then the electric current is turned ofl.

An anterior cut is made in the same way and a lateral cut through the lobe which is apparently most enlarged.

The length of the incisions depends upon the size of the prostate, but, as a rule, threc centimetres are sufficient for the posterior cut and two centimetres for the lateral cuts.

The after-treatment consists in keeping the patient in bed and passing a catheter if there is retention of urine.

Hamorhage is a rare accident, but can be controlled by the pressme of a large-sized catheter tied in the bladder.

It is not necessary to pass sounds, as the cuts show no tendency to close up.

The sloughs usually separate about the eighth to the fourteenth day, and some slight bleeding occurs; and if the operation has been successful in screring the obstruction the urine begins to flow freely.

The maximum of improvement is not attained, however, until after four weeks have passed, as by that time the bladder has recovered its tone to some degree, and the cicatrices in the prostate, formed by the healing of the incisions, have begun to contract and npen up the passage.

Selection of Cases.-Bottini's operation appears to be contraindicated in the cases of very large prostates the size of a small apple or larger, as the instrument can scarcely burn a furrow in them deep cnough to orercome the obstruction.

It is particularly adapted to the small, hard, fibrous prostates where there is a decided bar at the neek of the bladder.

The adrantages of the operation are that:-

(a) No anasthetic is required, and it is not accompanied with great pain.

(b) It is comparatively free from danger to life.

(c) It is applicable to the hard, fibrous prostates which cannot be successfully remored by prostatectomy and which do not atrophy after castration.

lirsults, as shown by Wossidlo's statistics:-

Cis:

110
CTRFN.

0.5
IMPROTED.

16
No Preit.T.

20
Dentus.

9

59 per eent. 13.6 per cent. 18.2 per cent. 8.2 per cent. 


\section{PROSTATOTOMY.}

By the term of prostatotomy is understood the simple division of a middle lobe, or eutting off a prominence which juts ont like a nipple and projects into the bladder, or the cutting of a $I^{2}$-shaped piece out of the prostate, in order to get a low-level channel from the bladder.

Prostatotomy may be performed by means of an opening into the bladder made above the pubes, but the perineal route is the one usually selected.

If the prostate is not more than three inches thick, it is possible, by means of an opening in the perineal urethra, to reach a projecting middle lobe, which may be readily divided with a knife, or, if it has assumed a nipple-like form, can be snipped off with scissors or an écraseur.

It is rarely possible by means of a simple prostatotomy to remove enough of the overgrowth of prostatic tissue to relieve the symptoms of obstruction, and the chief advantage which follows is from the benefit which the patient derives from the prolonged drainage of the urethra through a catheter and the attendant improvement of the cystitis.

According to Watson, 50 per cent. of cases subjected to perineal prostatotomy had a restoration of the function of the bladder and 10 per cent. died.

\section{PROSTATECTOMY.}

In this operation the entire substance of the prostate is enucleated from its capsule and removed through an incision.

Perineal Prostatectomy. - As a result of the experience gained in prostatotomies, in which a projection from a median lobe was torn off or a V-shaped piece was cut out of it, the next step in the development of the operation was fo enucleate the entire gland through a perineal incision.

In this operation, as it was done originally, the prostate wals approacher through a median perineal incision by which the uretha was 
opened into, as in external urethrotomy. The wound thus made, how. erer, was 100 limited in extent to afford ready access to the gland, and a transerse perincal incision two and one-half inches in length and curing aromint the rectum was adopted.

In one-1lird of the cases it was found, at the time of operation, that the distance from the junction of the membranous and prostatic urethra to the most distant point of the median enlargement within the blatder was more than three inches, and consequently the prostate was located out of reach of the finger, and enucleation was impracticable.

To obviate this difficulty Alexander and Nicoll practice making a suprapubic incision into the bladder, and, by means of two fingers introduced into the bladder, press the prostate down, so that it can be easily reached through a median incision in the perineum.

The advantage of opening the bladder above the pubes is not alone to press down the prostate so that it can be reached from below, but an opportunity is afforded to examine the cavity of the bladder thoroughly with the finger and to search for and remove a calculus, if present. If the stone should happen to be located in a saccular dilatation of the bladder, it would be impossible to remove it in any other way.

Technique of Alexander's Operation of Perineal Prostatectomy Combined with Suprapubic Cystotomy.-The bladder is first opened by mons of a suprapubic cystotomy. The patient is then placed in the lithotony position, and a grooved staff introduced throngh the urethra into the bladder. The membranous urethra is opened upon the staff by means of a median perineal incision. The forefinger of the surgeon's left hand is then passed through the suprapubic wound in the bladder, and presses the prostate downward into the perineum. The forefinger of the right hand is introduced through the perineal woml into the prostatic urethra, and its mucous membrane is torn throngh at one side. The finger is pushed through the opening thus made, and emuleates one of the lateral lobes of the prostate. The liece, after it is enucleated, can be seized with foreeps and withdrawn through the perineal wound.

The finger is then reintrodnced, and the lateral lobe of the other side and the middle lobe are enucleated and removed in the same way. The unly part of the urethra which is torn lies in front of the ejaculatory ducts. The perineal wound is drained through a large eatheter carricel through it into the bladder, and hæmorrhage is controlled by 
a gauze packing around the catheter. A drainage-tube is introducul through the wound in the bladder, and the bladder-malls sutunul around it. Both wounds are usually healed at the end of five weeks.

The adrantages which Alexander claims for his metirod are as follow:-

"I. The entire obstructing portions of the prostate are thoroughly removed through a perineal opening withont injury to the moncous membrane of the bladder or of the prostatic methra abore the opening of the seminal ducts.

"II. Hæmorrhage is rarely a serious complication.

"III. The most eflicient and thorough drainage of the blatder is secured.

"IV. The time required by practiced hands to perform the operation is comparatively short.

"V. The best conditions are left for a return of complete roluntary urination."

"The dangers of the operalion are:-

"I. Suppression of urine in those cases in which there is atvanced renal disease.

"II. Hæmorrhage, which is rarely serious and can always be controlled by packing the perineal wound with ganze about the drainagetube.

"III. Injury to the rectum. It is, however, only necseary to remember this danger to aroid it."

The results in Alexander's hands are as follow: Ont of 31 cascs operated upon, 3 deaths, 1 partial snecess, and $2 \gamma$ snccessful cases. in which the vesical function was restored and patients emptied their bladders completely.

Suprapubic Prostatectomy.-The removal of the prostate cland by means of a suprapubic cystotomy is considered by some surgeons to possess certain advantages over the perineal operation. The patient is saved any loss of blood from the perineal incision, and the operation can be very rapidly performed.

There is, however, great liability to lacerate the base of the blander if the prostate is at all adherent, but the chief disadrantare comrs from the urine soaking throngh the incision which is made in the latie of the bladder for the purpose of enucleating the prostate. and cansing suppuration of the surrounding tissues, and the hamorrhatec is often alarming.

The suprapubic operation can only be performed when the earrity 
of the biadier is fairly eapacions, for when the bladder-walls are thickcued and its carity very much contracted, this operation is impracticable.

The operation of suprapubic prostatectomy as originally performed by Belfield and McGill has been simplified in certain points by Eugene Fuller.

T'echnique of Fuller's Operation.-The patient is laid flat upon his back. The Trendelenburg posture is not adapted, nor is Petersen's rectal bag used. The bladder is filled with ten ounces of boric-acid solution, and a rapid suprapubic cystotomy is made.

The location and extent of the prostatic obstruction is determined by examination with the left forefinger. By means of a pair of long-handled scissors, with serrated edges, slipped along the left forefinger as a guide, an incision is made through the mucous membrane of the bladder, which lies above the prostate.

The cut exposes the prostatic tissue, and extends from the margin of the internal vesical opening of the urethra about one and onehalf inches in a backward direction. The serrated edges of the scissors prevent any free hæmorrhage from the incision.

Firm counter-pressure is made with the fist against the perineum, and the forefinger of the other hand is slipped through the incision in the bladder-wall, and by means of the finger the entire prostate is 'mucleated, en masse or piece by picce, depending upon the character of the growth.

After the prostate has been entirely enucleated the patient is put in the lithotomy position, an opening is made into the urethra through the perinemm, and a large-sized rubber catheter is inserted, through it, into the bladder for purposes of drainage.

Finally, after hot irrigation, the wound in the abdominal wall is partially closed with silk-worm-gut sutures, one of which includes the bladder-wall at the upper angle of the vesical incision and another one jases through the bladder-wall at the lower angle of the wound. In this way the bladder is liept in close apposition with the abdominal wall, so that there may be no leakage into the space of Retzius. A doulle drainage-tuhe is inserted into the bladder.

The chief alement of success in this operation is the rapidity with which it is performed, and it is also essential to use the smallest quanlity of olher with which anasthesia can be maintained, on account of thr lowerd vitality attendant upon the age of the patients.

The after-lealment consists in careful and thorough vesical irri- 
gation through the drainage-tubes and the administration of lares. quantities of distilled water by the mouth, for its diuretic ellect.

In order to guard against hypostatic pulmonary congestion firm lying in bed, the patient should never be allowed to lic long in one position, but should be carefully turned from side to side, and the shoulders should be elevated occasionally.

Results of Suprapubic and Perineal Prostatectomy. - It the time that the operation of complete removal of the prostate first came into prominence the objection was raised by Guyon, Socin, and Thompson, on theoretical grounds, that, even though the obstructing prostate he removed, the contractile power of the bladder has been so much inpaired that the patient will be incapable of spontaneous urination after the operation. The tabulated results of 150 prostatectomies, however, now demonstrate that in 75 per cent. of the cases restoration of the biadder function does take place, and that patients who were previously dependent upon the catheter are enabled to minate voluntarily again.

In spite of the good results attained through prostatectomy, there are certain grave objections to the operation. The subjects of enlarged prostate are always advaneed in years, and, as a rule, their vitality is at a low ebb. Prostatectomy is a serious operation and attended with a very considerable amount of shock.

In the days following the operation the patient is exposed to the dangers of ether-pnemonia, to which old people are liable, and to the risks of septicæmia from infiltration of urine into the wound, or nræmic poisoning from a failure of secretion on the part of the kidneys.

On these accounts prostatectomy should never be molertaken in the case of a debilitated, feeble old man, or in one who has marked atheromatous degeneration of the arteries.

Even in selected cases, although the results, as far as restoration of the bladder functions are concermed, are better than after the other operations, the mortality is higher, and ranges from 11 per cent. (Predal) to 20 per cent. (Watson).

Another contra-indication to prostatectomy is a hard, fibrous condition of the prostate. If the prostate is in this state, it is diflicult or impossible to enucleate it, and some other surgical procedure must be adopted. 


\section{CASTRATION.}

T'he danger to life and the dilliculty of performing the various operations of prostatectomy have led surgeons to scek for other means of remoring the olstructing prostate.

In 1893.1 . William White, of Philadelphia, first published the lesults of his experiments, showing that the removal of both testicles caused an atrophy and shrinking of the prostate.

Velpean and Sir Henry Thompson called attention, years ago, to the similarity between the fibromyomata oceurring in the prostate and those of the uterus. Following up this suggestion, White castrated a number of logs, and found that the operation was in every ease followed by a rapid atrophy, first of the glandular structures of the prostate and then of the muscular elements.

The theoretical grounds upon which White accounts for the diminution in size of the prostate are as follow:-

The prostate in the man, although not embryologically the true homologue of the uterus, is developed from tissues quite distinet from those which go to form the urinary passages.

The structure of the prostate and the uterus is similar, as they are both hollow muscular organs containing glandular follicles.

The normal growth of the prostate is in direct relation to the sexual life of the individual, and its overgrowth occurs at a period when the sexual life is fading out, but is usually not extinct.

The reproductive powers of life end sooner in the female than in the male, and, on this account, fibroid tumors of the uterus appear earlier in women than do prostatic growths in men.

The histology of uterine and prostatic growths are notably similar in both sexes.

The uterine tumors do not appear after the menopause, and if already present mulerog atrophy with the cessation of menstruation.

The prostate in men does not continue to increase in size after a certain time of life is reached, but has a tendeney to grow smaller.

In the female removal of the ovaries causes the uterine fibrony yomata to disappear, and the uterus itself undergoes an atrophy as well. and, in the male, removal of the testicles has a similar effect upon the prostate.

Diminution in Size of the Prostate.-The first effect upon the frutate of the remoral of the testicles is to cause a rapid disappearance uf the congertion, which reduces the gland somewhat in size. This 
slight reduction in bulk often relieves the obstruction and the matient can sometimes urinate spontaneously a few hours after the oferatins of castration

The experiments on dogs and autopsies upon mon have shown that a true atrophy of the gland takes place from absorption and di:appearance of its glandular structure, which necurs first and is followed later by the partial disappearance of the connertive-tis-ne stroma which lies between the glandular elements.

These changes may require a few weeks or from four to -ix months before they are completed; so that we should not be disappointed if after castration several months elapse before any good results are manifested.

The general indications for castration are the same as for any of the other operative procedures, viz.: "approaching break-down in catheter-life."

Castration, however, is particularly adapted to the cases of larce. soft, clastic prostates, congested with blood and containing a good deal of glandular tissue.

The prostates which have advanced to the second stage of hypertrophy, in which the glandular tissue has disappeared and has been replaced by connective tissue, causing the prostate to becomc hard and of a dense consistence, are unfavorable cases for castration, and, while good results sometimes follow from the decongestion of the orwan, the filorous residue of the prostate does not shrink after the operation.

Clinical Results of Castration.-Castration has now been done for the relief of hypertrophied prostate in hundreds of cases, and the results of the operation have been closely followed by many different observers. The statistics of 111 cases of castration, which were collected by White, showed that a rapiri atrophy of the prostatic enlargement occurred in 87 per cent. There was a disappearance or lessening of the long-standing cystitis in 52 per cent., a return of resical contractility in 66 per eent., and an amelioration of the most troublesome symptoms-viz.: inability to pass water. frequent urination, and painful urination-in 89 per cent. of the cases.

It will be noted that in Dr. White's table the resical contrictility was restored in 66 per cent.

Fenwick takes the ground that, if a blakler has been criplled for three years or more by severe cystitis and entirely incapable of being emptien except by the catheter, it is highly prohahle that. even if the prostate shrink, after castration, the muscular fibres of the blad- 
Jer will be so atonied that the catheter can never be dispensed with, althongh its introduction will be easy.

In commetion with this view it is interesting to note that Bruns reports 28 cases in which the catheter had been used from a few months up to two years and in which voluntary urination was restored in $2:$ cases.

In 20 cases in which the catheter had been used from two to twenty years spontaneous urination returned in 8 of them after castration.

'The cystilis is reported as improved in 52 per cent. of White's cases, and the improvement is accounted for by the possibility of the bladder being completely emptied of its residual urine, owing to the reduction in size of the prostate and also from the relief of the passive hyperemia in the prostatic plexus of reins and the nucous nembrane of the bladder-walls.

In 1897 Englisch published tabulated statistics of 202 cases of castration, which had been performed by various surgeons. Of these. in $14 ;$ only were the details stated with sufficient fullness to make them worthy of record. Englisch finds that the age of the patient has but little intluence upon the result, but the amount of prostatic obstruction, the quantity of residual urine, and the state of the kidneys bear a direct relation to the mortality of the operation. In these cases, as reported, of 106 patients operated upon, who suffered with retention of urine, 23 per cent. died, and, of 18 patients without retention, only $5 \%$ per cent. died. The state of the bladder and kidneys is a still more important ensideration in the result. Of 47 patients with severe eystitis who were eastrated, 20 died, 2 were cured, and 19 were improved; of 46 patients with slight cystitis, 4 died, 20 were cured, and 20 were improved. In 110 cases a successful result was shown by rerluction in size of the prostate and an improvement in the accompanying symptoms in 69 of them. A reduetion in the size of the prostate without an improvement of the symptoms ras noted in 6 cases. and an improrement in the symptoms without reduction of the size of the prostate in 27 cases.

The morlatity of castration was found to be 34 deaths in 220 cases. In 14 of these it was not possible to attribute death to the operation, and in the other 20 death was almost invariably due to advanced disease of the urinary organs.

In 1898 Albarran and Motz collected and published statisties of 121 enstrations. They divided the cases into four groups:- 
(a) Cases of dysuria, but without retention. Of 14 enses of prostatic hypertrophy with these symptoms, 7 were cured and $t$ were inproved.

(b) Cases of acute retention. Of 20 prostatics with aeute retention, 3 died soon after the operation and the other 17 quiclily recrained the ability to empty the bladder, and did not experience a relapse.

(c) Cases of chronic partial retention. Of $t 1$ prostatics with chronic retention which was not complete, 4 died after eastration, 19 were cured, 14 were improved, and in 4 the residual urine was not decreased in quantity, but the vesical tenesmus improved.

(d) Cases of complete chronic retention. Of 49 prostatics with complete retention, 10 died after the operation, 20 were entirely cured, 14 had a partial return of spontaneous urination, and in 5 there was no result.

In the acute cases of retention and dysuria the good results are attributed to the relief of the congested state of the prostate, and in the patients with chronic retention a diminution in the size of the prostate is brought about and at the same time the contraetility of the bladder is increased.

It should be borne in mind that improvement does not follow after every castration, and it is generally conceded that the hard, fibrous prostates are the ones which do not atrophy after a remoral of the testicles.

A careless diagnosis is responsible for a certain number of faihures.

Various local conditions, such as a narrow meatus, a long prepuce, stricture of the urethra, and particularly a vesical calculus, all produce symptoms resembling those resulting from an enlarged prostate, and, indeed, may be present as complications. Therefore before procceding to advise a castration all such conditions should be excluded by appropriate examination.

The ease and rapidity with which castration can be performed has also led to its being used as a last resource to relieve the distress of old men, who were nearly moribund and who were unfit to stand the slightest form of operative procedure. In such cases, of course, no relief can be expected from a castration, and the patient's ineritable death is only hastened.

Mortality of Castration.-On examining the statistical tables the death-rate of castration seems surprisingly high for such a simple operation. Of the $15+$ eases collected by Engliseh. 16 prer cent. तlied, 14 per eent. of Albarran and Motz's $15 \pm$ eases died, and 18 yer eent. of White's 111 cases resulted fatally. 
This hich death-rate may be explained by the fact that a number of paticuts we operated upon who were in a uremic or even dying mindion. White states that 13 of the fatal eases in his report died from existing kidney disease, and, if these are deducted, it will leave a leath-rate of only $r$ or 8 per cent., which he considers the legitimate mortality of castration. 'This riew is also borne out by Englisch's taliles. In 106 cases which he collected and who suffered from retention of urine, 23 per cent. died; while, of 18 patients castrated who had no retention, the unortality was only $5 \frac{1}{2}$ per cent.

His tables also show the relation which disease of the bladder and kidneys bears to the mortality. Of 47 cases who had severe cystitis, 42 per cent. died; while, of 46 cases with but a slight degree of cystitis, only $s$ per cent. were fatal.

In Albarran and Motz's cases, of the patients with acute retention of urine, 15 per cent. died; of those with chronic complete retention, $\because 0$ per cent. dicd; while with those of partial retention the death-rate was only 9 per cent.

Remote Results of Castration.-A small number of patients after heing castrated developed delirium, or a condition resembling senile dementia, and it was formerly thought that the removal of the testicles was responsible for this state, upon the supposition that the testicular secretion was necessary to the mental vigor and equipoise of a man. That riew is now abandoned, and the delirium which occasionally follows a castration is supposed to be either traumatic or uremic in origin, as a similar form of delirium is known to occur at times after any surgical operation upon an aged person.

From the opportunities which have been afforded in the Orient, from times of earliest antiquity, to observe the eunuchs, it is highly probable that the mere removal of the testicles will have but little infuence on the mental force of the man. Curvan states that, in the East, the eunuchs are shrewd and sagacious mentally and rigorous in body. with the exception of those who are degraded by practices of Eexual perversion. 


\section{VASECTOMY.}

The disinelination of patients to allow the remoral of their testicles has led surgeons to endearor to cause an atrophy of the prostate by means of ligating the spermatic cord through an incision in the serotum.

Experiments on animals show that, when the spermatie cord is divided, the prostate sometimes grows smaller. The decrease in size is accounted for by a lessening of the congested condition of the gland, which takes place immediately after the operation.

In two anatomico-pathological examinations of prostates marle some time after the operation, no atrophy of the gland was discernible, althongh the contractility of the bladder had been improved by the operation.

Albarran and Motz collected the following statistics of the operation of vasectomy: In 47 cases 7 deaths followed the operation. A diminution in the size of the prostate occurred in 21 eases, which was due to the effect of the operation in causing a decongestion of the gland. In some of the cases a subsequent examination showed that the diminution in size was only temporary, lasting about a month. Tasectomy exerted little influence upon the contractile power of the bladder. In 11 cases of acute retention 3 were enred and 5 improved. In 40 cases with dysuria, or incomplete retention, 4 were cured and 5 improved; and in 19 cases of chronie complete retention 3 were cured and 3 improved, 5 died, and 8 were without results.

From these studies Albarran and Motz conelude that resection of the vasa deferentia is often followed by a lessening of the congestion of the prostate, which canses its rolume to diminish temporarily, and evokes an improvement of the dysuria, the eystitis, or the retention, from which the patient suffers.

There is nothing to prove that the operation canses an atrophy of the hypertrophied prostate, or that in cases of chronic incomplete retention the residual urine is diminished if the operation is made at a time when the prostate is not congested. There are also no records to show that after the operation the bladder is able to empty itself. 


\section{PALLIATIVE OPERATIONS.}

In these operations no attempt is made to remove any portion of the protate. They are done for the sole purpose of draining the bladder, treating the eystitis, and avoiding eatheterization.

The bladder may be drained by means of

(a) Perineal opening. (b) Suprapubic cystotomy.

The perineal incision is the operation of choice when it is desired to drain the bladder temporarily for the sake of giving a respite to the urethra which has been irritated by the hourly passage of a catheter, or to remore blood-clots from the bladder which have originated from liamorihage into its carity.

In cases of serere and obstinate crstitis the perineal opening and continuous drainage serve to clear the bladder from the thick mucopurulent urine and to restore the vesical mucons membrane to a healthy state and the urine to its normal acid reaction.

The perincal incision is a less serious operation than suprapubic cystotomy; the danger to life is not as great, and an opportunity is also oflered to search the bladder with the finger, and, if a small stone is found. it ean be removed.

The prostatic urethra is stretched and dilated with the finger at the same time, and a large catheter is introduced through the wound and kept in the bladder for from one to three weeks, draining it of its residual urine and allowing it to be washed out and kept clean and aseptic.

'The drainage of the bladder gives great comfort. The patient is not aroused from sleep every few minutes to pass water. The tenesmus and pain on mrination are no longer felt, the cystitis subsides, and the prostatic adema lessens. Sometimes spontaneous urination follows. After the drainage is discontinued the introduction of a catheter beconles easy.

Unfortunately, however, drainage through the perineum cannot be kept up indefinitely. The posterior urethra becomes irritated and resents the presence of the catheter after a few days, and so much pain and spasm are caused by it that the catheter has to be removed.

In such cases, when the drainage is still required, we must have recourse to another mode of accomplishing it: i.e.,

Suprapubic Cystotomy.-An opening made into the bladder above the pubes gives an opportunity to inspect the cavity of the bladder and 
remove a calculus if present, and if the stone happens to be in a siccular dilatation it can never be found in any other way.

The suprapubic fistula answers very well as a permanent opening and is more easily managed by the patient than the jerineal opening. A tube is arranged to pass through the fistula, in the abdominal wall, into the bladder, siphon off the urine, and allow it to flow into a reservoir under the clothing (the Bloodgood hladder-drain). The patient wearing such an apparatus is able to be up and about, even to take long walks aud enjoy a fair amount of activity.

Puncture Through the Substance of the Prostate with a Trocar.In cases where there is an imperative necessity for draining the bladder, but for some reason the retention of a eatheter in the urethra is impracticable, drainage may be aceomplished by plunging a trocar into the perineum and through the substance of the prostate, as suggested by Reginald Harrison.

A catheter is introduced into the bladder through the cannula of the trocar and left in, and the canmula is withdrawn. A serious objection to this form of tumneling the prostate is the danger of the septic urine leaking into the substance of the prostate through the wound, and causing parenchymatous suppuration.

\section{CHOICE OF OPERATIONS IN HYPERTROPHIED PROSTATE.}

The knowledge which we possess at the present time, of the relative worth of the various operations, is too indefinite to enable us to lay down any hard-and-fast rules for the selection of the procedure best adapted to relieve a particular case.

There are certain conditions which differ in each individual, and which have to be carefully considered before selecting the operation which seems most applicable to the case in hand.

\section{POINTS FOR CONSIDERATION.}

I. General Conditions.-(a) The age of the pationt.

(b) The state of his general strength.

(c) The condition of his sexual power's.

(d) The state of his kidneys.

(e) The amount of atheroma in the arteries. 
II. Coudition of the Prostate and Bladder.-(a) The size and density of the jircstate.

(b) The form of the obstruction: whether it is in the form of a bar across the resical outlet, a tumor deflecting the urethra, or a nlass the size of an orange filling up the pelvis.

(c) The condition of the bladder in relation to its atony or hypertrophy.

(d) The quantity of residual mine.

(c) Catheterization, its ease, or if attended with pain and bleeding, and its necessary frequency.

(f) 'The severity of the accompanying cystitis.

III. Complicating Conditions.-(a) Tesical calculus.

(b) Stricture.

(c) Diabetes.

For the better selection of an operation it is useful to adopt the classification of prostatic cases suggested by J. William White.

Class A.-Patients with moderate enlargement of the prostate, who sulfer little or no pain, and with clear residual urine to the extent of three or four ounces. For these cases no operation is to be thouglit of, and they get on very well with methodic catheterism. But in time, as these difficulties increase, they come into Class B.

Class B.-These patients suffer with marked obstruction from the prostatic overgrowth at the resical outlet. The prostate ranges in size from one and one-half inches in diameter to three inches (about the size of a lemon). The residual urine may be eight or ten ounces in amount, or there may be complete retention. The cystitis is marked, and the urine is purulent, ammoniacal, and fotid. Operative interference of some lind is clearly indicated, and the rhoice lies between prostatectomy, castration, and Bottini's operation.

If the patient is strong and vigorous, with active sexual powers, and is free from atheroma and with sound kidneys, and if the hypertrophy is glandular in character, causing the prostate to be soft and clistic and of large size, prostatectomy may be considered. although it should be lon me in mind that even in skilled hands the death-rate rateges from 11 to 20 per cent.

If the patient is older and more feeble, and has atheroma or 
diseased kidneys, Bottini's operation or castration woukd be the operation of ehoice.

Bottini's operation appears to be indicated particularly in the patients with hard, dense, fibrous prostates, for castration has but little effeet in eausing this variety to shrink.

The soft elastic prostates give the best results after calstralion, although Bottini's operation may be applied in these casos also.

'The ehoiee between Bottini's operation and castration is sometimes determined by the form of the obstruction, which may be demonstrated by a eystoseopie examination.

Class C. - In this class the prostate is as large as a basc-ball or small orange. Retention of urine is nearly or quite complete, and eatheterization is difficult and painful. The lidneys are usually liseased, and atheroma is extensive. In these cases eastration probalily offers a better chanee of relief than any of the other opcrations.

Class $\mathbf{D}$ ineludes the desperate eases with enormous prostates and exeessive cystitis. The bladder is dilated and saecular, the retention eomplete, and catheterism diffienlt. These patients are advaneed in years, and suffer from chronie minary fever. The kidneys are diseased, and atheroma is extreme. Any surgieal operation is attended with great danger, but the patients' suffering is so great that they are willing to aceept any risk for the sake of relief. In these eases the choice of operation would lie between the establishment of permanent drainage of the blathler through a fistula, preforably suprapubie, or eastration.

Dr. White says: "Castration offers more hope of material benefit, with less risk, than any other operation, although, of course, the mortality is high. It oecasionally fails to do good; but, on the other hand, I have seen an improvement and many cases have been reported that were simply marvelous, eystitis ranishing and the power of voluntary urination returning in patients in whom such results would have been thought almost beyond the limits of possibility."

Diabetes is regarded as debarring all operations unless cathotorization is impossible. In sneh cases permanent drainage thromeh a suprapubic fistula affords the best means of relief.

For the purpose of presenting the statistical material which hats been eollected, in a tabular form. Wossidlo has arranged the tollowing table, in order to eompare the results of the rarions operattoms: 


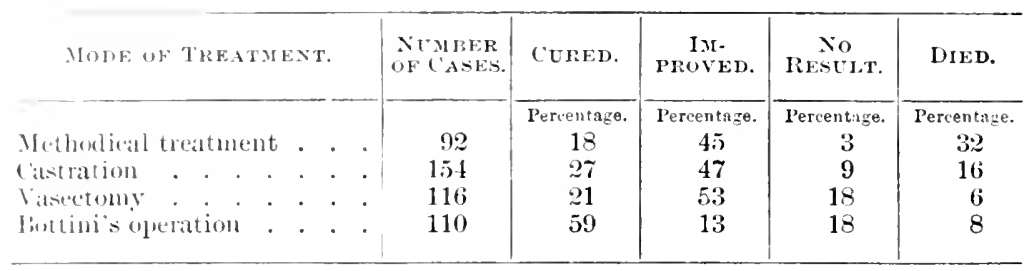

In the course of time, as our knowledge is increased through experience, it is fair to assume that, by a judicious selection of the operation for each individual patient, we shall be able to reduce the number of cases in which there is no result or but slight improvement, and that our efforts will be more uniformly certain of complete success than at the present time. 


\section{CHAPTER XV.}

\section{TUBERCULOSIS OF THE PROSTATE.}

Tre prostate is involved in nearly every case of genito-urinary tuberculosis. Of 26 cases of tuberculosis of the prostate reported by Socin, in 24 of them other genito-urinary organs were allected and only in 2 did the genito-urinary apparatus escape. In these two instances the lungs and bones were the seat of tubereular deposits.

Although Sir Henry Thompson denied that the prostate coull ever be the seat of primary tuberculosis, later investigators have proved conelusively that it may be, and Desnos and Krzwicki even go so far as to state that, in their opinion, in most eases of genitourinary tuberculosis the prostate is the organ which is first infected with tubercle bacilli, and from that focus the infection subsequently travels to the adjacent structures. This point is important to bear in mind in connection with the operative treatment, which will be considered later.

The time of life at which tuberculosis is most apt to fasten upon the genital organs is that period at which sexual activity is most highly developed, and consequently we find that our patients are usually between twenty and forty-five years of age.

Predisposing causes play an important rôle in the etiology of tuberculosis of the prostate. Anything which induces prolonged and oft-repeated congestion of the posterior wrethra weakens the resistance of the tissues; consequently a tubereular outbreak is more liable to occur in the person of a young man who has practiced some form of sexual abuse or has been the subject of a prolonged attack of gonorrhœa of the posterior wrethra. But this in itself is not sullicient to cause the disease, and to bring this about the tubercle bacilli must be actually introduced into and develop in the substance of the gland.

As to the modes of infection, the micro-organisms are most frequently brought to the prostate in the blood-circulation. oftun from a tubercular deposit in some distant organ. In other cascs they may be taken into the body throngh the respiratory or alimentary tricts, and, passing along with the blood-current, be ultimately deposited in the prostate. 
The innculation of bacilli, howerer, may be direct, and be occasioned by an infected catheter or through coitus with a tubereulous female, or the prostate may be infected by a process of extension from some neighboring organ.

\section{PATHOLOGY.}

A deposit of tubercle takes place in the substance of the prostate, and either one or both lobes are affected. The tubercular nodules are multiple from the beginning, or else soon become so, and they enlarge until several coalesce, when they break down and form ahecesses.

Cnless remored by operation, the pus bursts through into the rectum or urethra or even the hypogastrium, and multiple fistulous tracts are formed. In rare instances the cheesy mass becomes the seat of calcareous changes, or the fluid portion of the mass is absorbed and the residue is encapsulated, and a healing of the lesion results.

It is important to note that the bladder and seminal vesicles are always involred sooner or later in the course of the disease. The epididymis is also affected, although in many cases this organ is the first attacked, and the infection extends to the prostate subsequently.

\section{SYMPTOMS AND COURSE.}

If the process begins in the central part of the prostate, no definite symptoms are caused; but if the nodules are located superficially, and cause a bulging of the prostate toward the rectum, a sense of reight in the perineum and difficulty in defecating is experienced.

If, on the other hand, the tubercular foci lie close to the urethri, the symptoms are those of posterior urethritis, viz.: frequent and urgent urination, accompanied by a nuco-purulent discharge from the urothra and shreds in the urine.

There is no distinct pain after the act of micturition, but a feeling as though the bladder were not fully emptied.

Defecation spermatorrhea sometimes occurs if the deeper-lying prostatic lubules are infiltrated with nodules, and another form of sccretion from the meatus is occasioned by the breaking down and discharge of small alsscesses through the urethra.

IIrmaturia is a frequent symptom, and is not constant, but intermittent. The blood comes at the end of urination, and is not due 
to an ulceration of the urethra, as formerly sumprein, int nur. to the congestion of the prostate.

In uncomplicated cases pain may be excruciating, and is mmetimes so severe that it overshadows all the other symptoms.

As the disease progresses the bladder is almavs attecter. Thic is announced by the occurrence of pain after urination and tenesmus. and as the cystitis grows worse the bladder symptoms bocome the marked feature in the case.

In most cases of tuberculous prostatitis death is caused by an ascending infection, involving first the bladder and subsequently the kidneys, or the lungs may be attacked, or a general miliary tuberculosis may be established.

In a few rare instances the disease remains limited to the prostate; an abscess forms, which breaks and discharges, the carity cicatrizes, and a curc follows.

\section{DIAGNOSIS.}

On rectal examination the tubercular prostate will be fouml enlarged in one or both lobes. The enlargement is distinctly nodular or lumpy, and at first of a stony hardness. After the abscess form: points of softening with fluctuation can be rearlily felt.

It is often extremely difficult, indeed sometimes impossible, to determine whether the enlargement is in the prostate or involves the? seminal vesicles, for these different organs may be so blended touther by the inflammatory exudation that the lines of demareation camot be defined. On this account it was formerly supposed that erery tubercular process in this region was confined to the prostate, whereas we now know it to be true that the tubercular process may attack prostate and resicles together, or either organ be involved alone.

The gonorrhoal inflammations of the prostate. chronic prostatitis, or the acute suppurative form resembles in physical signs the tuberculous disease, and it is impossible definitely to establish the diagnosis of tuberculosis until the presence of tubercle bacilli lias been demonstrated in the secretions. These may be collueted ly expressing, with the finger in the rectum, the socrotions from the prostate gland; and the bacilli are also generally plocent in the discharge from the meatus which so often exists.

If no tubercle bacilli are found in eximining the servelions, guinea-pigs may be inoculated with the liwharess. ant. if the litg develops tuberculosis, the diagnosis is, of course. cstablisherl. 
In cases of general tuberculosis it is usually safe to consider every entargement of the prostate tuberenlar in character, and the (m) lifticulty in diagnosis arises in the cases where the prostate is the seat of primary tubereulosis.

\section{PROGNOSIS.}

The prognosis is, of course, of the gravest, although when the tuberculosis is limited to the prostate alone spontaneous cure sometimes occurs through healing of the cavity after the abscess has formed and burst.

Lnfarorable elements in the case are an hereditary predisposition to tubereulosis and a tendency for the disease to extend and involve other organs.

\section{TREATMENT.}

The general treatment consists in endeavoring to vitalize the tissues by means of a life in the open air or a prolonged sea-voyage, abundance of nourishing food, and the administration of codliver-oil, creasote, guaiacol, and other antitubercular remedies.

There is a difference of opinion as to the advisability of beginning local treatment early. In general, it is better to avoid instrumentation, for the reason that the local resistance of the tissues is reduced, and infection of the bladder with other micro-organisms readily oecurs.

On the other hand, instillations into the posterior urethra in the early stages have their adrocates. Guyon advises sublimate sol, 1-5000 to 1-3000; and iodoform in glycerin is warmly recommended ly Berlieley Hill. Everyone is agreed that nitrate of silver uniformly acts badly, and its use is contra-indicated.

After cystitis has set in the principal indications are to control the pain and tenesmus, but these matters have been considered in another section. (See "Cystitis.")

Lnder the head of operative treatment may be considered, first, the suggestion of Ifofmam. which was to inject 10-per-cent. emulsinn of indoform and glycerin into the substance of the prostate by means of a long needle thrust in through the perineum. This urocelure has rever gained firor, and is to-day praetically abandonerl.

I few yeal's ago, when the dictum of Sir Henry Thompson, that "tuluerenlosis of the prostate was never primary, but always secondary 
to deposits elsewhere," was accepted as final, it was thompht win... to attempt to extirpate the diseased prostate, and the rute was only to operate when pus had formed, in order to evacuate the contents it the abscess.

At the present time the opinion has changed, and the operation of laying bare the prostate by a semilunar incision curving aroum! the rectum and removing all the diseased tissue with a cur.tte commends itself as a rational and conservative procedure, and one which is likely to bring about a radical eure, when the disease is limited to the prostate alone.

When the prostate is secondarily affected, and deposits exist in other organs, the indication for operation is not so clear, but even in these cases an operation is likely to save the putient the misery and suffering from a prolonged course of suppuration of the prostate, with the slow formation of fistula. 


\section{DISEASES OF THE KIDNEYS.}

\section{CHAPTER XVI.}

\section{MOVABLE KIDNEY.}

Ax unnatural morability of the kidney occurs in two forms:-

(a) Movable kidney is more frequent in its occurrence than the other form. In this variety the kidney moves about freely behind the peritoneum, as it lies in a sort of pouch or cavity formed within its own fatty capsule.

(b) True floating kidney lies closely surrounded by its fatty capsule, and is supplied with a mesonephron, which is attached by one end to the spinal column, but which is so long that it allows the kidney to float about freely in the peritoneal cavity. The extent of the excursions of the kidney depends only upon the length of its mesonephron.

\section{ETIOLOGY.}

Morable kidney oceurs more frequently in females than in males, Lindner finding that 1 out of every 5 or 6 women examined were so affected, and the right kidney is more frequently movable than the left.

True floating kidney is either caused by a congenital mesonephron or it may exceptionally be the last stage of a movable kiliney.

Various reasons are suggested for the preponderance of movable kidney in the female, the ehief of which are laxity of the abdominal walls as a result of child-bearing. On closer scrutiny, the facts do not uphold this theory, for a movable kidney is found as often among virgins and nullipare as among women who have borne children.

It is probable, however, that the cause of movable kidney in loth sexes is the absorption of the fat which surrounds the kidney and acts as a cushion and support to it, as a consequence of the enuciation ocemring in wasting diseases.

If the fat is absorbed, a slight blow or muscular strain, such $(\because 26)$ 
as the exertion of romiting, may be enough to hosen the kithey trom its attachment and permit it to move about more or leso fruels.

\section{SYMPTOMS.}

The symptoms of movable kidney are, of enurse, obsure. Gastro-intestinal symptoms, such as flatulence and dysperpia, ale generally present, and have been accounted for by the supposition that the kidney in its abnormal situation exerts pressure upon the duodenum and narrows its lumen, thus causing partial retention and fermentation of its contents.

Edebohls considers these symptoms due to pressure and traction upon or stretching and irritation of fibres of the solar plexus, lying in the abdomen and belonging to the sympathetic nervous system, because it would seem that the theory of obliteration of the lumen of the duodenum is insufficient to account for the symptoms.

Edebohls calls attention to the other symptoms which are generally caused by floating kidney, as follows: Pain is felt in the epigastrium, which is not increased by pressure, and is located somewhere to the left of the median line at or near the free border of the left costal cartilages. General nervousmess in greater or less degree exists, and is usually accompanied by cardiac palpitation and habitually rapid action of the heart.

These patients are also unable to sleep or to rest comfortably while lying upon the left side.

In true floating kidney with a long mesonephron in addition to the presence of the above-mentioned disturbances the patient is conscious of abdominal pains, of a dragging or pulling character, and the sensation is felt as if some foreign body were moring about in the abdominal cavity, particularly after a sudden muscular exertion, or upon rising up surdenly after lying down.

The pain may be only wearing in character or it may oceur in paroxysms and be agonizing. Its onset is sudden, and it is apt to follow fatigue or active exertion. The pain in some cases is due to attacks of local peritonitis.

Nervous disturbances, hypochondria, melancholia, and hysteriil usually occur, either as reflexes or from disturbances of nutrition. The secretion of urine by the misplaced lithey is, in general, not interfered with. 


\section{DIAGNOSIS.}

The diagnosis is not difficult to make in thin subjects with relaxed abdominal walls. By palprating the flank between the fixed border of the ribs and the crest of the ilium the displaced kidney can be felt between the two hands.

In fat subjects the diagnosis presents greater difficulties. If the kidney has been displaced into the pelvis, it may be mistaken for an orarian or fibroid tumor; but the kidney may be differentiated by replacing it with ease in its natural position in the flank, while the attachment of its mesonephron prevents its complete deseent into the pelvis.

If the mesonephron is shorter and the kidney is found lying in the abdomen, it might be looked upon as a distended gall-bladder, enlargement of the spleen, or a tumor of the omentum.

\section{PROGNOSIS.}

A kidney which once becomes movable never again becomes firmly fastened in place, except by operative interference.

A misplaced kidney, however, is, in general, not fatal to life, and, if death oceurs, it is usually the result of exhaustion from chronie dyspepsia, continued pain, and nervous depression, although death is sometimes due to malignant disease developing in the displaced organ.

\section{TREATMENT.}

The symptoms of movable kidney may be ameliorated by lying in bed, by the Weir Mitchell treatment, and by massage and electricity; but none of these methods offer much prospect of permanent relief (Edebohls).

A simple bandage of elastic webbing without any pad over the lidney and which, encircling the whole abdomen, makes as much pressure as the patient can comfortably bear, helps to support all the aldominal viseera and with them the kidney. Such a method may be tried before proceeding to operation, but it is generally found to be ineffective in holding the lidney in place.

On account of the difficulty of retaining the kidney in position, nephrorrhaphy, or fixation of the kidney, is advised by Edebohls as the first resort in patients with movable kidneys which produce decided symptoms. 
Nephrorrhaphy is an operation which is not attenderl with much danger to life and it is generally successful in fixing the kirney and relieving the symptoms. Delvoie reports 215 cases operater on by fixation, of which 135 were cured, 30 improved, 25 unimproved, 2010 relapsed, and 5 died.

Nephrectomy, or total removal of the kidney, has no justification when the kidney has only a small range of motion, and the opuration is accompanied by a very high mortality.

In cases of true floating lidney with a long mesonephron it may be impossible to fix the lidney in place. Such a contingency would demand nephrectomy by the lumbar incision if possible, and, if this is not practicable, by means of a laparotomy, although the mortality is much higher with the abdominal route than when the kidney is removed through the flank and without opening the peritoneal cavity.

\section{RENAL CALCULUS.}

The formation of stone in the lidneys is of frequent occurrence, and often precedes resical calculus. Stones composed of uric-acid crystals are most common, and the next in point of frequeney are oxalic stones. Phosphatic calculi are rare, and are caused by the alkaline decomposition of urine as a result of pyogenic infection.

The stones are found in the pelvis of the lidney, and may be single or may be in hundreds.

\section{ETIOLOGY.}

The cansation of kidney stone is generally the gouty diathesis (see "Vesical Calculus"). A few crystals are agglomerated in one of the renal tubules upon some substance, such as a blood-clot, coagulum of pus, or roughness of the wall, which serves as a nucleus. The agglomeration is washed out into the pelvis of the liduey, where it becomes the nucleus of a calculus.

The stone, if single, may be adherent to the walls of the pulvis of the kidney, or it may be movable and act as a ball-ralve, closing the mouth of the ureter. The urine is dammed back, in conserpence causing hydronephrosis. 
Infection with micro-organisms usually oceurs, and suppurative nuplititis follows.

\section{SYMPTOMS.}

A stone may be present in the kidney for years without causing much diromfort. as the symptoms are due, not to the mere presence of a forcin body, but to obstruction. They are as follow:-

(a) Attaclis of renal colic.

(b) J'ain.

(c) Hamaturia.

(d) T'yuria.

(c) Disturbances of urinary function.

(f) Gastro-intestinal disturbances.

(.) Passage of fragments of calculi.

Attacks of renal colic are strongly indicative of kirney stone, although typical paroxysms may be induced by other causes, and not infrequently renal stone has been diagnosed and operated for and its absence denonstrated on cutting open the kidney.

Iienal colic is caused by the stone being forced ont of the pelvis of the kidney and entering the meter. An attack comes on abruptly, and is characterized by agonizing pain, which is felt in the loin, and radiates down into the testicle and along the inner side of the thigh.

The pain may also radiate through the abdomen and chest, and be very intense in the back. In serere attacks nausea and romiting occur, the pulse is feeble, the shin is covered with a cold sweat, and the patient is in a state of collapse.

In lighter cases urination is frequent, and the urine discolored with blood, or the patient may be unable to empty his bladder.

In rare cases the secretion of urine may be entirely suppressed cither from (a) reflex disturbance of the secreting kidney; $(b)$ extensive disease of the other lidney, whose ureter is not blocked; $(c)$ where only one lidney exists in the body.

In these cases uramic symptoms develop after a week, and death talics place within a fortnight after the obstruction.

The pain and disturbance of ureteral colic may last for a few minutes or for a number of hours. and usually ceases abruptly as the stone either drops back into the pehis of the kidney or makes its escape from the lower end of the ureter and enters the bladder.

The stone occasionally lycomes permanently fixed in the ureter, in which casc, after some time, the walls give way slightly around it, 


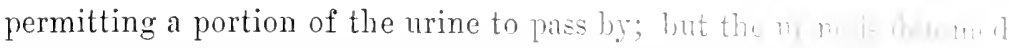
back upon the kidney, and hydronephrosis is deruloped.

Pain is felt over the affected kidney, which is mereasul ly moti.n. jarring, and pressure over the side.

The sensation is that of a feeling of weight, rather than of acute pain, but the patient is liable to have paroxysms of acute pain. which often occur at night, when he is at rest in bed.

The pain radiates along the ureter and into the traticle, and often causes contraction of the cremaster muscle, with drawing up of the testicle on the affected side. The pain may be referred to the healihy kidney or to the bladder, thigh, or calf of the leg.

Hæmaturia occurs most frequently when the stome is passing through the ureter, but may appear when the stone is in the pelyis of the kidney.

The bleeding may come on in a slight amount, giving the mine a smoky color, or the urine may be free for days until, after some sudden exertion or a prolonged ride, a considerable amount of hleeding takes place.

Pyuria occurs only after suppurative disease of the kidney has occurred, but this is usually established in time.

Disturbances of Urinary Function.-The irritative effect of stone in the kidney causes it to secrete an excessive quantity of urine by day when the patient is moving about, but at night. when he is quiet in bed, the secretion of urine is normal in quantity.

As a result of blocking the ureter with a stone, the urine may he diminished in quantity or entirely suppressed for a time. If it persists after the attack is past, it is clear either that the mreter of the functionating kidney is blocked with a stone and that the other kidney is so much diseased that it is not capable of secreting or else that there is only one kidney present.

Occasionally the obstruction derelops insidiously withont attracting attention until symptoms of mrenia set in.

Gastro-intestinal disturbances may be reflex in oring or $11 \mathrm{ny}$ result from imperfect elimination by the diseaced kidneys. T) spensia, romiting, and epigastric tenderness may easily cause the comdition of the kidneys to be overlooked.

Passage of fragments of calculi is wery often aheent, ol the flitsments are overlooked by the patient. but when puresut it is af ereat

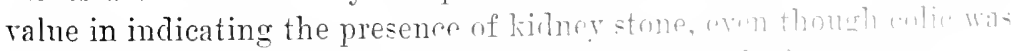
not caused by the pasage of the fragnents throlt- 


\section{DIAGNOSIS.}

A positive diagnosis of renal calculus is difficult to make, and many cases are on record in which the kidney was incised for the purpuse of remoring a stone when none was present.

The most characteristic symptoms of kidney stone are passage of gravel or fragments of stone, attacks of typical renal colic, hæmaturia, and, in time, pyelitis.

Himaturia and pain are often caused by tubercular or malignant disease of the kidney, and oxaluria and strongly-acid urine occasion a dull ache over the lidneys or even paroxysms of pain, which are sometimes accompanied by hæmaturia.

Spinal caries of the lower dorsal vertebre and locomotor ataxia may simulate the pain of lidney stone.

During an attack of renal colic it may be impossible to distinguish between the passage of a gall-stone or of a stone through the ureter.

Before attempting any operation it is, of course, essential to determine if both kidneys are affected or if the disease is confined to one side only. The location of the pain and tenderness on palpation throw some light as to which side the disease is located upon; but the question may be settled positively by catheterization of the ureters.

The x-ray has been of great use since its introduction in diagnosing cases of lidney stone, and is now regarded as one of the indispensable diagnostic aids.

As a final resort in the cases where stone is suspecterl and the patient's health is failing, it is justifiable to make an exploratory incision into the kidney by lumbar nephrotomy.

\section{PROGNOSIS.}

A stone may remain in the pelvis of the kidney for years without causing any serious disability, and only at times inducing transient pain or hæmaturia; but the patient is continually exposed to the danger of obstruction of the ureter and suppuration of the kidneys from pyogenic infection.

If a fragment of stone passes into the ureter, it usually escapes into the bladder, but if it become impacted in the ureter, disorganization of the kidney ultimately takes place, and the same is true if suppuration of the lidney occurs. 


\section{TREATMENT.}

Attacks of renal colic are sometimes cut short hy a molonged hot bath and a full dose of morphia hypodermically.

In giving opium it is necessary to exercise cantion and nut give too large a quantity, for as soon as the stone is reletserl and slips out of the ureter the pain ceases, and the patient may be orerenme by the effect of the drug.

In cases which do not respond to morphia it may be necestary to secure relaxation by means of anasthesia with chloroform or ether.

In the intervals of the attacks the general diathetic condition of the patient upon which the formation of stone dupends should receive proper attention (see "Treatment of Stone in the Bladder").

But little success has been attained by efforts at dissolving stones. Piperazin in 5-grain doses taken in a pint of water three times a day is thought to have some effect in dissolving the albuminous franework of a uric-acid calculus, and phosphatic stones may perhaps be softened and broken down by Urotropin.

The most important point in the treatment, in addition to resulation of the diet and general hygiene, is to keep the urine abundint and of low specific gravity by drinking freely of pure spring-or thistilled water.

Although a stone may remain for years in the pelvis of the kitney without danger to life, certain conditions-such as a deterioration of the general health, blocking of the ureter, or pyogenic infection of the kidney-call at once for the operation of nephrolithotomy.

This is indicated when the pain is persistent and severe, calling for the constant use of anodynes and accompanied by freyuent attacks of renal colic and gradual emaciation and loss of strength.

Through a lumbar incision the kinney can be exposed and cut into, on its outer convex side, sufticiently to admit the fingur into its pelvis, which can be thoroughly surched and, the stone fomm and removed.

If the kidney has been the seat of prolenced suppuration and has undergone complete disorganization, nephrectomy (untire remoral uf the lidney) may be indicated. 


\section{PYELITIS.}

In suppurative pyelitis, often called surgical kidney, the pelvis of one kidney may be attacked alone or both kidneys may be affected.

The pathological change, if due to an ascending infection, begins in the pelvis of the kidney, which becomes distended with pus, and if the ureter is blocked so that the pus cannot be discharged into the bladder, in conrse of time the interstitial secreting substance of the kidney is utterly destroyed and the organ is converted into a mere shell surrounded by its capsule and filled with pus. This condition is called pyonephrosis.

In pyelonephritis the suppurative process involves not only the pelvis, but the secreting structures of the lidney as well.

A number of small abscesses form between the tubules, and as they increase in size the intervening portions of tissue break down, so that sereral of the abscesses become merged into one.

The secreting parts of the lidney involved in the process are destroyed and the secretory function of the kirney is, of course. impaired.

After destruction of the kidney-substance the fluid portion of the

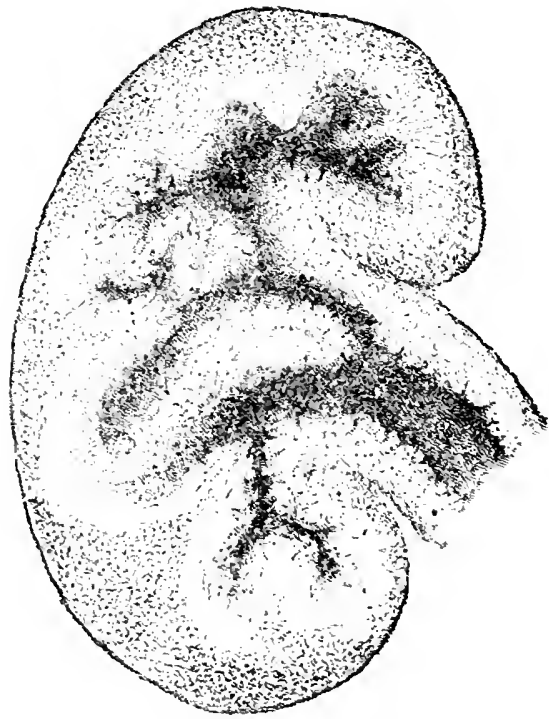

Fig. 73.--Tuberculous Pyelonephritis. 
pus is often absorbed, and upon antopsy made up of several sacculi containing grayish, jutty-he un. . Wh are often mixed with calcareons material.

\section{ETIOLOGY.}

Suppuration of the kidney is always caused by micro-oriani-nuof which the most frequent forms are the stalylylococcus anreus and the colon bacillus, and in tubercular pyelitis the tulsercle lacillns.

The modes of infection may be (a) ascenting, caused by the jhisage of micro-organisms from the bladder upward through the ureter. and $(b)$ homatogenous, in which case infective rmboli occurning in sur sis, tuberculosis, or the infectious ferers are conveyed to the kidnu through the blood-current.

Predisposing Causes.-The most important and frequent canse of pyelitis is inveterate cystitis from the retention of urine in the blathler behind a stricture or enlarged prostate.

The urine is dammed back upon the kidneys, cansing a distension of the pelvis, and infection with micro-organisms which are converent through the ureters from the bladiler readily takes place.

Renal calculi in themselves do not excite pyelitis, but the protlonged mechanical irritation to the kidney which their presence canses lowers its resisting-power and permits the entry of germs.

Infectious diseases-such as typhoid ferer, pnemmonia, scariatini. small-pox, and general tuberculosis-lead to the formation of infectious emboli, which are carried through the general blook-circulation and are often deposited in the lidney.

\section{SYMPTOMS.}

As most cases of pyelitis are secondary to some other condition the symptoms are obscured by those of the priniry discalse.

There are, however, certain definite sympoms which alwas atecompany pyelitis:-

(a) Changes in the Urine.-Pus is alwars present in connrideralle quantities, although it often disappears for a ferr hours. firmu a temporary blocking of the ureter.

Blood is frequently present, and is increased ly artivity an the part of the patient. It may be miformly diswminaterl thrught the urine or appear as long clots formed in the nreters. 
The quantity of urine secreted is increased, and is from two to three times the normal amount. Its reaction is persistently acid, unless cystitis is established, when it becomes ammoniacal, and the symptoms of cystitis-pain, tenesmus, and frequent urination-oceur.

Jlicroscopic examination of the sediment shows pus, albumin, small quantities of epithelial cells from the pelvis of the kidney, considcrable mucin, and numberless micro-organisms.

If pyelitis is dependent upon a renal ealculus, in addition to the pus-cells fragments of the calculus may be present, and crystals of uric acid, urates, or triple phosphates are always found. The quantity of desquamated epithelimm is greater, and some blood is present.

(b) Fever of an intermittent type often accompanied by chills, which may be easily mistaken for malaria, is a prominent symptom.

(c) Emaciation and progressice deterioration of health are results of the fever and toxæmia. In old men the appetite and strength fail, the tongue becomes dry and brown, and the pulse feeble and intermittent.

(d) Pain is rarely a prominent symptom, unless caused by a kidney calculus, although a dull aching, which is subject to exacerbations, is experienced.

It is by no means infrequent that the pyelitis remains latent without causing any symptoms, until the operation of litholapaxy, internal urethrotony, or the commencement of catheter-life causes it to become active.

In these cases the symptoms which arise are partly due to want of elimination, on account of the destruction of the secreting portion of the kidney, and partially from urinary fever, caused by the absorption of miero-organisms and toxins into the circulation from a wound in the genito-urinary tract.

The symptoms consist in a liminution in quantity or complete suppression of urine. The urine contains blood, often in abundance. The temperature rises, and is accompanied by feeble pulse and great prostration. Delirium and coma set in, and are followed by death.

\section{DIAGNOSIS.}

The chicf lifficulty in maling a diagnosis of pyelitis is to distinguish it from eystitis.

To this end pain and tenderness over the kilney and perhaps a histinct tumor may be noted on palpation. 
The acidity of the urine, which persists smeral hily witer : is passed, and the sudden fluctuations in the quantity of fus are sinnificant of pyelitis.

A manœurre which is sometimes of use, in dilierentiatimg leetween cystitis and pyelitis, consists in washing ont the lilarkler thwroughly, and then waiting for fiftcen minutes; the urine which hats accumulated in the bladder during that time is then drawn off with a catheter.

If the kidneys are healthy, but cystitis is present, a small anount of pus will appear in the urine when it is drawn off, but if the bladder is healthy and pyelitis exists, the urine will contain a comparatively large quantity of pus.

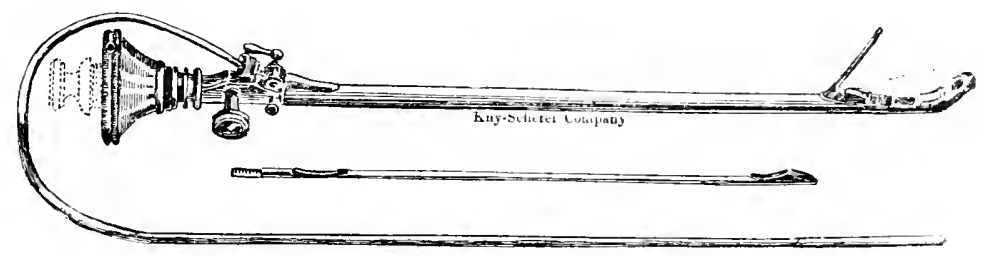

Fig. 74.-Nitze's Cystoscope for Catheterizing the Ureters.

The cystoscope is an important diagnostic aid, and by its use cystitis can be excluded and the discharge of purulent urine can often be seen coming in jets from one or both ureters.

By means of the catheterizing cystoscope the ureters may be catheterized and the mine from each kidney collected separatcly, or Harris's segregator can be used for the same purpose. In women the Kelly method of catheterizing the ureters can be employed.

\section{PROGNOSIS.}

The prognosis of pyelitis depends, of course, upon its (anust. When it occurs in the course of an. infectious fever, recovery anderally takes place. Tubercular or suppurative prelitis may terminite by inspissation of the pus, or it may break through the alluthe of the kidney and set up perinephric abseess, or make its way thrombly the? skin of the loin, or break into the intestime or huges.

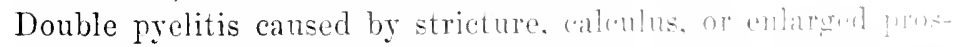

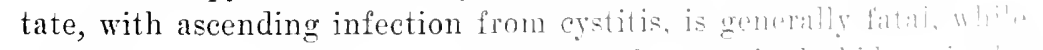

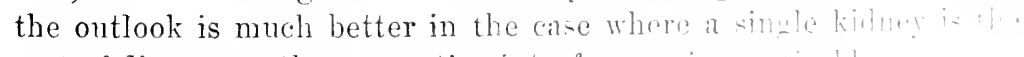
seat of disease, as then operative interference is mantinitu. 


\section{TREATMENT.}

In mild cases of pyelitis occurring in the course of an infectious discase the patient should be kept in bed, put on a milk diet, and large quantities of distilled water given by the mouth. Crinary antisintics-Urotropin and salol-are indicated and citrate of potash -hould be administered to diminish the acidity of the urine as it lies in the pelvis of the kidney.

The surgical treatment inchudes the removal of any obstruction to the frce escape of urine from the bladder, relief of the cystitis, the removal of renal calculi, and the evacuation of collections of pus in the kidney, nephrotomy and under certain conditions nephrectomy, or the removal of the kilney itself.

In pyelitis of gonorrheal origin and in all acute cases which after a short time begin to show improvement, Caspar and Kelly suggcst catheterization of the ureters and washing out the pelvis of the kidney with 3-per-cent. boric-acid or 1- to 2-per-cent. nitrate-ofsilver solutions.

The indications for surgical operation are intermittent pyuria with fever during the intermissions and septicæmia, even though the pus constantly escapes through the ureter.

The opcration indicated depends upon the extent of the destructive process in the kidney and whether one or both kidneys are affected.

I. One Kidney Alone Diseased.--(a) In the case of an abscess of the lidney, from a punctured wound or a renal calculus, it is desirable to drain as early as possible, in order to prevent destruction of the kidney-structures.

(b) If the entire kidney is infiltrated and riddled with abscesses, nephrotony is first performed and the pus evacuated. It often happens that the patient's condition improves and the sinus heals up and the remains of the lidney can be left in place to be of some degree of service as an eliminative organ. In many cases, howerer, the kidney-structure has undergone so much disorganization that its extirpation is indicated, and, after the pus has been evacuated by an incision, the kidney may be removed a few days later by nephrectomy.

It is, in all eases, considered safer to allow an interval of a few days to elapse before removing the kidney entirely, except in cases of tubercular pyelitis, where the kidney should be entirely removed at the priming operation. 
II. Both Kidneys Diseased.-In such conditions nenllw..th is out of the question, and in feeble old men, who art sufturing frm stricture or enlarged prostate, no operation ean be undertaken which holds out much prospect of recorery.

If the patient is in better general condition, evacuation of the pus by incision (double nephrotomy) may relieve the septic conclition, and, if the kidneys are not too much disorganized, they may be able to carry on their eliminative function sufficiently to maintain life.

\section{HYDRONEPHROSIS.}

When, from some mechanical obstacle in the ureter, the urine is prevented from flowing out from the pelvis of the kilney, retention of the non-purulent urine occurs, and the pelvis and calyces

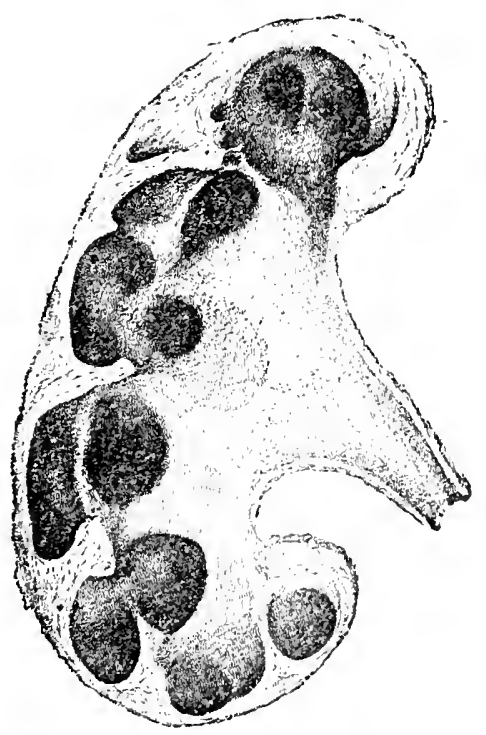

Fig. 75.-Hydronephrotic Kidney without much enlargenunt.

of the kidney become enormously dilated. Atrojhy of its surputing substance takes place, with the formation of distinct cysts, whith may attain to a very great size. 


\section{ETIOLOGY.}

Congenital Causes.-The ureter may be entirely absent, or obliterated in some part, or the obstruction may derelop after birth, on account of a tristing of the ureter or the formation of a ralve-like fold, cansing partial or complete closure of its lumen.

If an abnormal insertion of the ureter exists either at its origin from the pelvis of the lidney or at its entrance into the bladder, the angle of the insertion of the ureter may be so acute that it is compressed and its lumen closed when the patient is in the standing position.

Acquired causes operate either by compressing the ureter or obstructing the outflow of urine and causing it to be dammed back upon the kidneys. Tumors of the ovary and uterus, prostatic hypertrophy, and atony of the bladder will produce this effect at times.

A rery frequent canse of hydronephrosis is a renal calculus, acting as a ball-valve, as it lies in the pelvis of the kidney at the mouth of the ureter, or if it enters the ureter and becomes fixed at any point in its course.

From any of these causes the obstruction may be complete, so that no urine can pass through the ureter; or it may be incomplete, and a portion of the urine passes through into the bladder.

Intermittent hydronephrosis occurs chiefly as a result of a movable kidney, as changes in the position of the kidney cause bends and twists in the ureter, and in consequence stagnation of the urine in the pelvis of the kidney, and ultimately dilatation with cystic formation.

The sac becomes completely filled, and then, if the obstruction is temporarily remored, it is emptied, and a large discharge of urine occurs into the blatder, and the hydronephrotic tumor disappears.

The obstruction in the ureter returns and the sac gradually retills, and after a varying time is again emptied.

The process of aceumulation and discharge is repeated indefinitely mless the mreter hecomes completely and permanently blocked, which nsmilly oceurs in the course of time.

\section{SYMPTOMS.}

The smutoms of hrolmephrosis are rague and indefinite, and com-int in the furmation of a fluctuating tumor in the flank, which 
may be large enough to fill the entire abkominal waty the with frequent urination, and a diminution in the quantity of mune par-wl.

The symptoms caused by intermittent hylronephrois ane enenally occasioned by a movable kidney, in which condition the unetur is obstructed by being bent at an angle.

The attacks are aecompanied by violent pain and diminution in the quantity of urine and may easily be mistaken for an attack of renal calculus. When the kidney falls hack into its normal place the ureter is straightened out, the pain ceases suddenly, and large yuintities of urine are discharged.

\section{PROGNOSIS.}

Hydronephrosis may remain stationary for years. but the pressure of the acemulated fluid leads, in time, to an atrophy of more or less of the parenchyma of the kidney-substance, although the secreting structures are nerer entirely destroyed.

The affection is often complieated by infection with pyogenic organisms, and the case becomes conrerted into one of yyclitis, with its attendant dangers.

Large sacs have been known to rupture into the abdominal cavity and cause peritonitis, and, in a few cases of intermitrent hydronephrosis, spontaneous eure has oceurred.

\section{DIAGNOSIS.}

The diagnosis presents many difficultics, although a history of the sudden disappearance of a tumor coincident with the discharge of large quantities of urine is eminently sugacestive of intemiftent hydronephrosis.

An ovarian tumor is very liable to be mistaken for hychmophrosis. The distinguishing points are the situation of the livhny tumor in the flank, with the colnn or small intestine in front of it.

Exploratory puneture of the immor may throw some light unum the case, by furnishing a fluid containing urea or min ald, hut then ingredients of urine often disappear ly aborption. and the lingin resembles that contained in any simple eyst. Catheterization of the ureters may settle the question of liamosis by dompuntatimn if

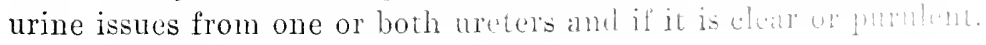




\section{TREATMENT.}

It is soldom possible to remove the obstruction in the ureter and resestablish the flow of urine through it, except when due to morable lidney or pressure upon the ureter from an abdominal growth, and the various attempts to do away with the obstruction by massage and ureteral catheterization, ete., have been rarely crowned with success.

In double hydronephrosis incision of both saccular kidneys by lumbar neplnotony, with an interval between the two operations, and the establishment of a permanent fistula, although eausing great inconvenience to the patient, aflords the only hope of arresting the destruction of the secreting portions of the kidney and saving the life of the patient.

In unilateral hydronephrosis incision and drainage through the loin (nephrotomy) is the operation of ehoice, and later on, if the annoyance of the fistula becomes unbearable and the other kidney is found to be healthy, nephrectomy may be done upon the diseased kidney and the fistula allowed to elose.

Before nephrectomy is undertaken it may be desirable to endearor to remore the obstruction in the ureter by catheterization, or the adrisability of transplantation of the ureters may be considered.

The operation of aspiration, or lumbar puncture, although formerly practiced, is selrlom employed to-day, except in the cases where an operation is strongly indicated, but for some reason nephrotomy cannot be performed at the time.

In certain cases of intermittent hydronephrosis a permanent cure is said to have followed the operation of lumbar puncture.

When hydroneplnrosis depends upon a movable or floating kidney, mephrorrhaphy is indicated, and, when the kidney is fixed in place, the accumulation of urine and orerdistension of the pelvis of the liduey may cease. 


\section{DISEASES OF THE TESTICLES.}

\section{CHAPTER XVII. \\ ECTOPY OF THE TESTICLE.}

THE testicles are developed in the abrominal cavity of the foetus. About the fifth month of fotal life they begin to descend, and pass through the inguinal canal into the scrotum, arriving there about a

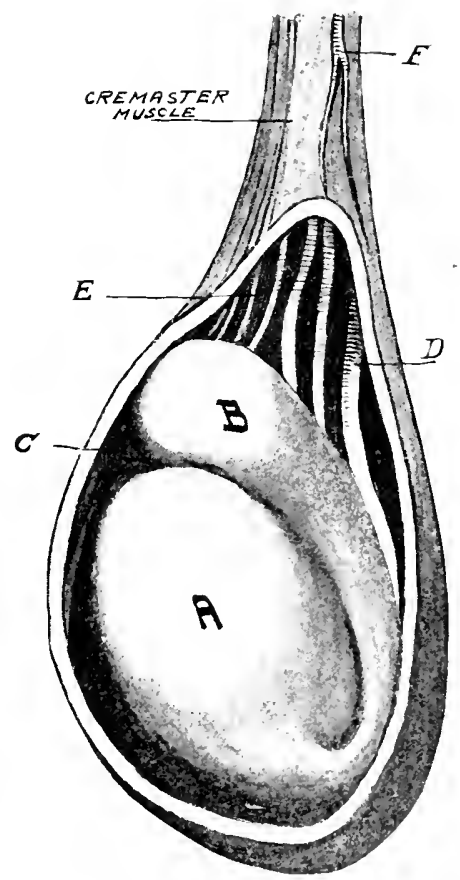

Fig. 76.-Testicle and Epididynis Exposed hy Cutting away Part of the Tunica Vatgalis.
$A$, Testis.
D. Tas Deferent.
$B$, Epididymis.
E, spermatio Artury and Vins.
$C$, Tunica Vaginalis.
$F$, Artery of curd. 
noonth before birth. One or both testicles may fail to follow the Iurmal course, and may be retained:-

(a) In the abdomen (eryptorchism).

(b) In the inguinal camal.

(c) The testis may take an aberrant course and be found lodged under the skin of the abdominal wall, the thigh, or perineum.

\section{ETIOLOGY.}

The causes which operate to prevent the normal descent of the testes are obscure. It may be accounted for, howerer, by assuming that the extcrnal inguinal ring is of too small a size to allow the testicle to pass through it, or that the ressels accompanying the spermatic cord are too short to allow the cord itself to be stretched sufficiently to allow the testis to reach the bottom of the scrotum. If the testicle is held by a long mesorchium in the abdominal carity its mobility may be so great that it slips past the opening of the inguinal canal without entering it.

The rearing of a truss, on account of a hernia, in a case where the descent of the testicle has been delayed after birth, will also prerent the testis from arriving at its normal place in the scrotum.

The causes of cruro-femoral and perineal ectopy are still more obscure. It is thought, however, that an overderelopment of certain bands of the gubernaculum will have the effect of drawing the testis to one side and thus occasioning its aberrant course.

\section{RESULTS.}

The results of ectopy are impairment of the growth and derelopment of the testis, so that it remains undersized, but probably possesses the porrer of forming spermatozoa, unless its structure is destroyed by attacks of inflammation, which are very liable to occur. After the testicle has been disorganized sterility, of course. follows.

\section{COMPLICATIONS.}

Iernia is a very frequent accompaniment, and if the testicle lies in the inguinal canal it interferes with the wearing of a truss, so that strangulation of the hernia is very liable to occur.

The testicle when not lying protected by the thighs, in the ecintum. is very liable to be struck and bruised, and the ectopic tesijcle rarely escapes sereral attacks of tramatic inflammation. 
It is also subject to gonorrheal inthumnim of a gonorrhea from the posterior urethrit.

Malignant disease is very apt to occur in thr a.tum. and is predisposed to by the attacks of inflammatione to whin? testicle in this situation is so liatile.

\section{DIAGNOSIS.}

The diagnosis is made by (a) the absence of the testicle from the scrotum; ( $($ ) the detection of a smooth. oral, solt tumor in the inguinal canal, which mores up and down when the pationt coughts and strains.

The testicle should not be mistaken for a hemia, which often complicates this condition.

\section{TREATMENT.}

In intra-abdominal ectopy, or eryptorchism, it is imposible to rectify the condition by operation, as the spermalic cort is ton shout to admit of placing the testis in the scrotum.

In addition, as the testicle is protected from trammatiom, inflammation does not occur in it, and the dangers of destruction of its function, with consequent sterility or malignant degeneration, do not occur. and the chief reasons for operation do not exist.

In inguinal retention it is often posible by means of gentle manipulation to push the testicle into its uroper place in the sarotum, and it may be retained in position by wearing a proper truse, which closes the ring and prevents its retmrn.

If this procedure fail to retain the testicle in place, and the child is over six years of age, a surgical operation should be performed. for the reason that the testicle does not reach its full size and functional development while in an almormal situation. From its exposed lucation it is also constantly lialule to blows and injuries. These learl to repeated attacks of inflammation, cansing sterility. and also predisp.... to malignant discase. The selcetion of the opreratim will depend upun the history of the case. An operation to replace the testicle in the scrotum is only ardrisable before the occurrence of attarks af inflimmation. After attacks of inflammation have occurred. the surenter structure of the testicle is destroyed. ant the orem is remlerent wh.....

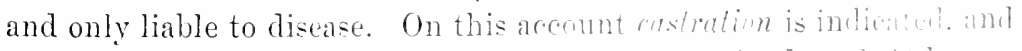

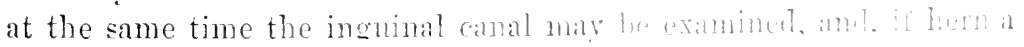
is also present, it may he radically cured and the cantal chent. 


\section{MALIGNANT DISEASE OF THE TESTICLE.}

It is diflicult to draw a distinction between benign and malignant growths of the testicle, becanse histologically the tumors are nearly always mixed formations composed of fibrous, myxomatous, sareomatous. and cartilaginous elements associated together, and tumors of the testicle which are apparently innocent are often followed by secondary deposits in the adjacent lymphatic glands and other organs.

\section{VARIETIES AND CLASSIFICATION.}

Sarcoma may be composed of spindle- or round-cells, or may, in its early stages, appear as numerous small cysts, filled with elear or lark fluid, seattered through the substance of the gland.

Carcinoma is usually of the soft, or encephaloid, variety.

Lymphadenoma, chondroma, fibroma, myxoma, and osteoma are also occasionally met with.

The clinical history of sarcoma and carcinoma, which are by far the most common forms, may be considered together.

The age at which the disease usually appears is from fifteen to forty-fice years, but sarcoma is occasionally found in very young children.

The disease begins in the glandular epithelium of the tubes or in the comnective tissue between the tubes, and increases in size, involving the whole body of the testicle.

The tumor formed is smooth and uniform, until the tunica albuginea breaks down, and after this occurs the growth feels irregular or nodular, with areas which are soft and fluctuating. The tumor increases more rapidly in size after rupture of the tunica albuginea and often becomes enormons. The skin of the scrotum sloughs and allows parts of the testicle and granulations to protrude through the opening, forming fungus testis.

The spermatic cord enlarges from the infiltration of its tissues by the new growth, and the adjacent lymphatic glands become infected and enlarged.

The veins of the scrotum swell, and the lower extremities become redematous from the pressure of the pelvic and lumbar glands upon the iliac veins.

The general health fails, the patient becomes cachectic and ema- 


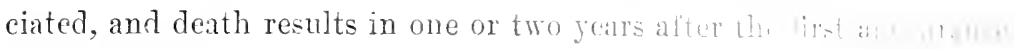
of the growth.

\section{PROGNOSIS AND TREATMENT.}

The great majority of tumors of the testicle are cither mal innut. tubercular, or syphilitic, and it is always desiralibe to try the rifect wit inunctions of mereury and large deses of iodide of potakh, for ten day. if there is any suspicion of syphilis.

If there is no improvement at the end of that time. castration should be at once performed.

If this is done early enough, the discase may be permanently cured, but unfortunately it is not uncommon for the lymplatic glanis in the pelvis to be involved. and a recurrence of the distase cliten takes place within a year after the operation.

\section{TUBERCULOSIS OF THE TESTICLE.}

The testicle is frequently the seat of tuberculosis, which always begins in the epididymis and may subsequently involve the borly of the testicle.

The epididymis may be affected $(a)$ primarily, which is most frequent; (b) secondarily from a tubercular deposit in one of the otler genito-urinary organs; or $(c)$ in consequence of a general tuberculosis.

\section{CHANNELS THROUGH WHICH TUBERCLE BACILLI ARE CONVEYED TO THE EPIDIDYMIS.}

When the epididymis is affected primarily, the bacilli are introduced into the general blood-circulation and carried directly to the epididymis by the spermatic artery.

When the infection in the epididymis is secondary, it is fleuguently derived from the seminal vesicles. These are often atfectent hy tritrculosis, as the result of tuberele bacilli which were introlum inte thet urethra during coitus.

After the tubercular process is establishen in the which the bacilli are carried along the vas deferene and lowlen in the ethitity mis

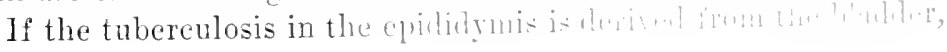


prostate, or other adjacent organs, the infection is usually carried by means of the lymphatics.

There are certain predisposing cuuses to tubercular infection, such as hereditary tendency to eonsumption and such local causes as the prolonged congestion from erotic excitement, an attack of gonorrluea, or slight tramatism. These causes all probably operate in the same way, and act by lowering the resistance of the tissues and permitting the tubcrcle bacilli to take efieet.

The time of life at which the testicle is most liable to be attacked is during its period of functional activity, from the age of puberty until past the fiftieth yoar.

\section{COURSE.}

One or two small nodules form in the head of the epididymis. Occasionally they remain latent for years or may beeome encapsulated and converted into fibrous tissue. As a rule, however, the nodules grow and coalesce until the whole epididymis is so much enlarged that it surrounds the testicle. After a time, the tubercular mass softens, beconce cheesy, and breaks lown.

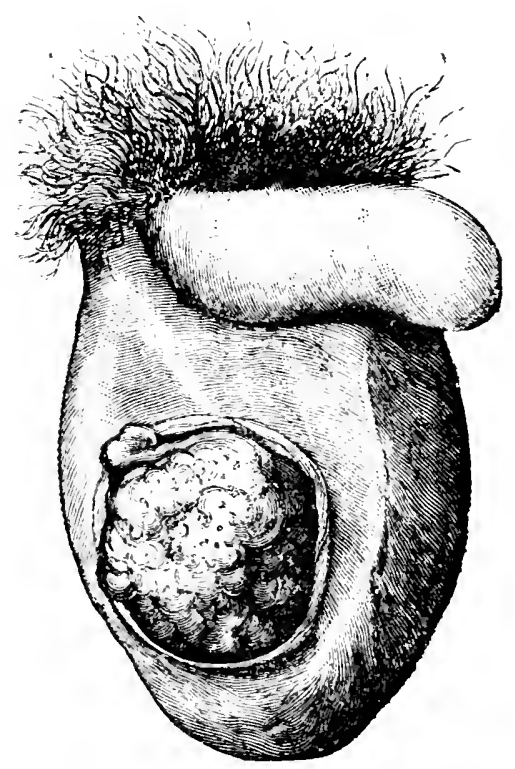

Fig. 77.--Ifernia or Fungus Testis. 
The skin of the scrotum lying orer the nulutes is atiatimi to the testicle, glued fast by the inflammatory alhesions, and the fus in discharged through an opening in it from the tuberentar afseces. bits ing a fistula.

The disease seldom limits itself to the epididymis, but, il let alone, spreads to the testicle. This occurs in three-fourths of the cases, as shown by autopsy.

After the abscess in the epididymis or testicle has opened, a considerable amount of attached skin, lying orer the nodule, may slough away, leaving an opening in the scrotum through which a mass of new granulation tissue, growing from the testicle or tunica alluginea, may protrude, forming hernia testis, or fungus testis.

In the earlier stages of the disease, before abscess-formation, the tunica vaginalis is affected by the inflammation, and, if an excess of fluid is secreted by its walls, hydrocele may be present.

On the other hand, adhesive inflammation may take place, and the sac of the tunica raginalis become obliterated.

Occasionally purulent collections containing tuberele bacilli are found in small cavities, circumscribed by the adhesions.

The vas deferens is always affected in time, and shows small localized nodules in its continuity, which are most liable to be located at its extremities,-i.e., near the seminal resicle and the epididrmis- the intermediate portion being free.

More rarely the cord is uniformly thickened, with a general diffuse tubercular infiltration.

The other testicle in time becomes affected, and the diseate rins its usual course.

Alchough tuberculosis of the testicle uswally rums a rery slow and insidious course, the nodules developing gradnally until the formation of pus, rare cases are occasionally met with in which the discase runs a very acute course, called by Rechus galloping consumption of

\section{the testicle.}

The nodules which had existed quietly for a time from some cantu take on a very rapid growth, and cascation and ahiress-formation ore-vur in about three weeks. In this form the terticle itsolf is aluats at-

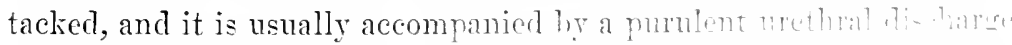
containing tubercle bacilli. The discharen may nrighinto spuntancously or it may begin as a gonoriluon from during coitus. 


\section{SYMPTOMS AND DIAGNOSIS.}

Tulerenlar nodules may exist in the epididymis, and remain latent for months, without causing a $r_{1} y$ discomfort, and, if discorered, they are uslially found accidentally.

blecumfort does not occur until inflammation begins, with its fain, swelling, and subsequent abscess-formation.

In the early stages examination shows one or more small nodules of a peculiar stony hardness to the touch located in the head of the epididymis and along the cord.

Later in the discase, after the nodules have become cheesy or broken down, the skin is found to be adherent over a circumseribed tumor, with hard walls and a central softening, which may yield fluctuation if pus is present.

Although the nodule may be actually located in the epididymis, the enlargement of this part of the organ is so great that it may surround the body of the testicle, giving rise to an appearance as though the nodule were in the testicle itself.

Suspicions as to the tubercular nature of a growth should always be aroused by the history of an epididymitis coming on without any apparent cause or after a slight injury, and, if hard nodules are found in the seminal resicles or prostate, the diagnosis of tuberculosis is sufficiently establisher, and may be confirmed by finding tuberele bacilli in the contents of the cheesy nodules.

Lnder treatment, the serere inflammatory symptoms of epididymitis quickly subside, and are nsually followed by a general softening of the tmmor and formation of abscess. differing in this way from a simple epididrmitis, which, after it is over, leaves a hard and thickencel epididymis.

\section{PROGNOSIS}

It is a very rare occurrence for tuberculosis of the testicle to become permanently cured without operation, even under the most farorable climatic influences.

When a cure docs oceur, it is through a process of encapsulation and fihroid degeneration, but it often happens that the process of encap-ulation only closes un the infective material for a time. and under favoring conditions the deposit again hecomes active and continmes its usual conrse of cascation. ahscess-formation, and infection of remote oreans.

Tuluremlar nodules, however, may remain latent for many years 
before abscess forms, or mintil a slight trunm w

rhoea stirs them into activity.

After suppuration the discase rmos a libit on

a fatal termination either by inducing tutherentis is n an.

or by the exhanstion and ferer incident to the whin purating fistulæ.

\section{TREATMENT.}

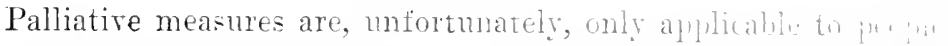
of means, who are able to lead an ont-of-door life ur take a long wa-

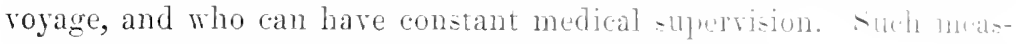
ures may retard the caseation of the nodules if begun in the arition stage while the deposits are sullil and hard.

As soon as the deposits begin to solten and beconne cherey. sulgical measures should at once be resorted to, as waiting for resuluim, which never occurs, is only a waste of valuable time, anl allow- the extension of the disease to remote organs.

In the case of hospital patients, who are poorly nomrithed and badly housed, and who cannot have suitable climatic surround nus, the nodules should be remorerl at once.

\section{OPERATIVE TREATMENT.}

Erasion, or curetting, is the operation which is applicable to the cases where one or two small nodules exist in the epirlidynis. The softened area should be opened and the cuntents well scrapenl out with a sharp spoon, iodoform rubbed in, and the carity lacked and allowet to heal by granulation.

Castration is the operation best alipted to the antrancenl ans...

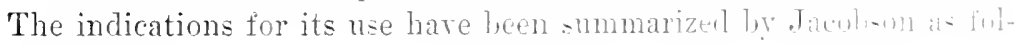
lows:-

(a) When erasion has faited in lesions of the enidily mis

(b) When discharging fistula are present or are numerome.

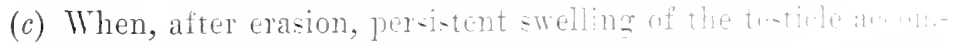
panied with night-sweats and los of fleth is unche

(d) When fungus testis exists or when the howly uf the the involved.

(e) In the presence of purulent lycilrumente

Castration is need in two nifferent chow:

Class A.-In primary tulurentw. 
(1) onc bricle and has not cxtended too high along the cord, and when the hiather, prostate, and resicks are not affected. In such a case a reasonalib hope may be entertined that the disease may be permanently cranticated from the body. If the seminal resicles are affected the indication for castration is not so clear, although, if the deposits are small and of recent date and the patient's general condition favors rapid healing, removing the testicle with its diseased nodules may retard the development of the tubereular foei located elsewhere.

Class B.-In cases where other organs of the body are tubercular and a cure is impossible, hygienic measures alone are the only treatment applicable. An exception should be made to this rule when the testicle is disorganized and the scrotum riddled with sinuses discharging pus. Here castration is indicated to relieve the patient from the drain of the exhausting discharges and from one source of his discomfort.

In the operation of castration the infiltrated skin and cellular tissue should be removed. The spermatic cord should be divided as high up as possible, if necessary laying open the inguinal canal and removing the cord as far as the internal ring.

The cord should be tied with a double ligature through it, as a single ligature is liable to slip off and cause fatal hæmorrhage.

\section{SYPHILIS OF THE TESTICLE.}

The testicle is attacked by spphilis in the late secondary and tertiary periods, which presents itself in two forms:-

(a) Interstitial or diffuse form consists in an infiltration of the comnctive tiscue betwcen the tubules, which becomes converted into a hard, fibrons induration, which compresses and destroys the tubules, and an atrophy of the entire olcan results.

(b) Circumscribed or gummatous form is a deposit of gummatous nolules varying in size from a pin-head to a hen's egg. After they have enlarged to a considerable size the mechanical compression nit the cortex causes a checs degeneration of the substance and a confraction of the nodule, or the centre becomes softened and is discharwer thromeh an ulcerated opening in the skin of the scrotum.

The cpilingmis may be involver secondarily in both forms, and 
hydrocele nsually occurs from an effusion of fluid into the sac of the tunica vaginalis.

\section{SYMPTOMS AND COURSE.}

The body of the testicle enlarges slowly and insidionsly, without causing any pain and it often becomes as large as an orange.

In shape its outline is regular, although the protuberance of a circumscribed gumma may sometimes be felt. Its consistence is lense, and it feels heavy. 'The spermatic cord, as a rule, is not thickened.

The scrotum is not affected until after the gumma brealis down, when it becomes adherent, inflames, and ulcerates, and fungus testis forms.

The disease is seldom bilateral at the beginning, but the other testicle may be attacked later.

Sterility only results from advanced disease in both organs, as a part of the secreting substance of the gland is spared.

\section{DIAGNOSIS.}

Syphilitic testicle is liable to be mistaken for tubereulosis, neoplasms, gonorrhcal epididymitis, or hæunatocele.

-The distinguishing points in syphilis are the regular outline and smooth, hard surface, and the fact that the cord is not enlarged, and in addition the history of a past attack of syphilis.

The diagnosis may be confirmed by the effects of antisyphilitic treatment, and this should be tried in every case of tumor of the testicle before proceeding to operation, if there is the slightest question of a previous syphilitic infection.

\section{PROGNOSIS.}

Under appropriate treatment syphilitic infiltration is absorled, and it is surprising to note how quickly large swellings disappear, and the function of the testicle is restored.

In untreated persons-particularly in tubercular, alcoholic, and weakly individuals-the gummata break down and dischrore and hernia testis follows, but even neglected cases, which furcult vilceration of the scrotum and large fungus testis, eencrally hat in from fom to six weeks under antisyphilitic treatment. 


\section{TREATMENT.}

The treatment consists in a general course of mercurial inunctions and the internal administration of iodide of potash in increasing doses, running it up to $1 / 2$ ounce per day, if necessary, to cause absorption of the newly-formed tissue. 


\section{HYDROCELE, HAEMATOCELE, AND VARICOCELE.}

\section{CHAPTER XVIII.}

HYDROCELE.

Before the testicle descends from the abdominal cavity in the foetus it is preceded by a process of peritonem, which makes its way through the inguinal canal and forms a pouch in the serotum called the tunica vaginalis testis. After the testicle has desconderi it lies behind the ponch, and is adherent to it.

Under ordinary conditions the opening in the pouch which formerly communicated with the abdominal cavity is closed. If it remain open, congenital hydrocele is said to exist. If the opening closes, the testicle is provided with a shut sac, lying in front of it and partly surrounding it, which serves as a protection against injuries, and allows of a certain freedom of motion.

\section{ACUTE HYDROCELE.}

ETIOLOGY.

Acute hydrocele is usually the result of a contusion of the testicle or a punctured wound of the sac of the tumica ratinalis. It may also occur from an extension of inflammation from the epididymis or testicle, occurring in the course of gronorithoa or other infectious disease.

\section{PATHOLOGY.}

Its pathology consists in an accumulation of seroms Huil. whith is occasionally stained with blood in the sac. and al depnsit of lymph upon its walls. 


\section{SYMPTOMS.}

The symptoms consist in œdema and redness of the scrotum, accompanied by an elastic painful swelling, which is tender on pressme over the testicle.

If much fibrin is deposited, crepitation is sometimes observed. Acute hydrocele terminates either in $(a)$ recovery in two to three weeks, with the formation of adhesions within the sac and a thickening of its walls; (b) in suppuration; or $(c)$ it may become chronic.

\section{TREATMENT.}

The treatment consists in rest, with suspension of the scrotum and the local use of an ice-bag or hot application. After the acute symptoms hare subsided the patient can walk about, wearing a suspensory bandage. If much fluid is present in the sac and tension is extreme, aspiration is required.

\section{ACUTE PURULENT HYDROCELE.}

It occasionally happens that a fibroserous hydrocele becomes purulent instead of resolving, or suppuration may occur early as a result of an infected puncture of the sac, or an extension of a suppurative process from the testicle or epididymis.

In such a case the contents of the sac, instead of being clear serum, is composed of pus. Such a condition is apt to result in involvement of the peritoneum or a general septic infection, unless the pus is evacuated by a free incision and drainage.

\section{CHRONIC HYDROCELE.}

Chronic hydrncele is generally confined to one side, and ordinarily oceurs beiween the twentieth and fortieth years. although children are occasionally born with it. It generally begins insidionsly, althongh it may follow an acute attack.

It is supposed to be a mere passive process of transudation de- 


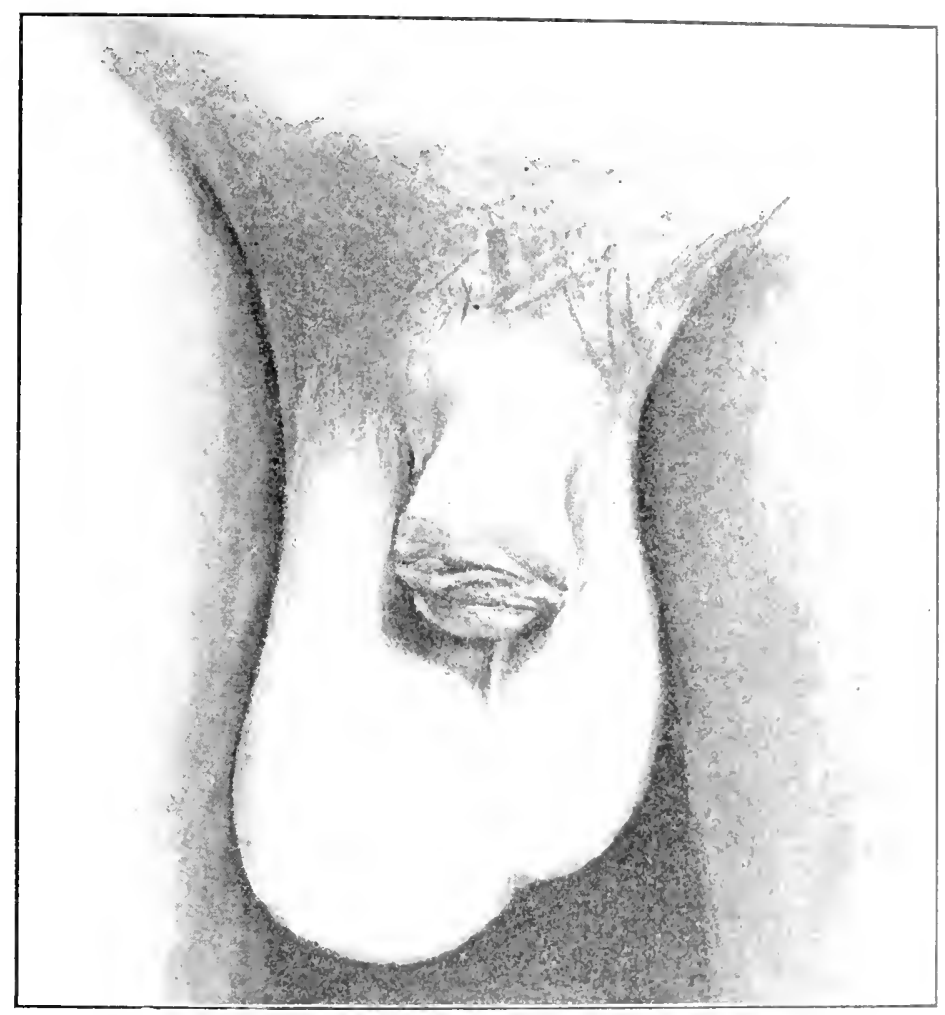

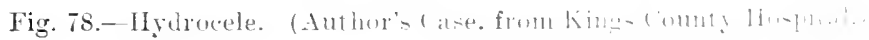





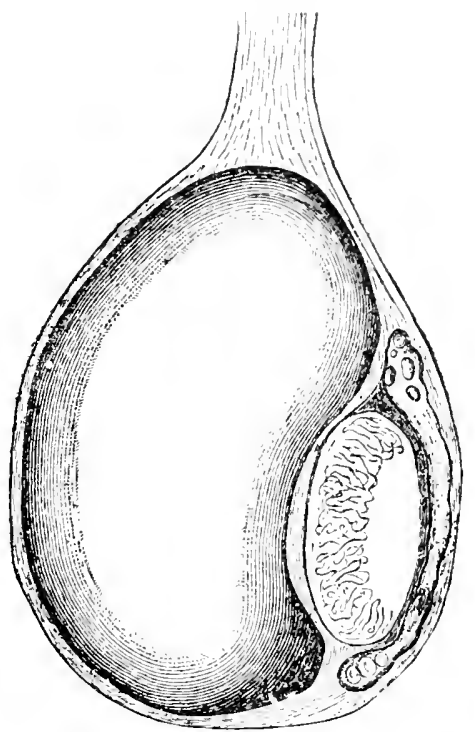

Fig. 79.-Tertical Section of Simple Hydrocele.

pendent upon the state of the blood-vessels and circulation, when it occurs as a primary disease.

\section{PATHOLOGY.}

The quantity of fluid which the sac contains is variable, from a few ounces to one, two, or eren three quarts. Its color is usually clear and watery, but it may be greenish or bloorly. If it contain a sarge number of desquamated epithelial cells and lencocytes, it appears turbid, and blood gives it a reddish or brown color.

In rare cases the fluid is white, and resembles mill, firm an admixture with lymph (hydrocele chylosa), and in the tropics, and in cases of true elephantiasis, filiaria are found in the milk-like content: of the sac.

In long-standing cases of chronic bydrocele the tunica raginalic is thickened, and may be calcifier in portions, and the tricticle and epididymis are hard and atrophied.

Sometimes adhesions form, and the sac is convertert into a number of distinct compartments. In other cates small fithere or fithcystic bodies are attached to the wall or lie loweng within the silc. 


\section{SYMPTOMS AND DIAGNOSIS.}

Hydrocele causes no symptoms exeept those which arise from the inerease in size and weight of the scrotal enlargement.

On palpation, the tumor is found to be pear-shaped and elastic to the touch, dull on pereussion, without impulse on coughing, and it cannot be reduced and returned into the abdomen.

Attention to these points will usually differentiate a hydrocele from a hernia, hæmatocele, neoplasm of the testicle, or a hydrocele of the cord.

An additional point in diagnosis is the translucency of the

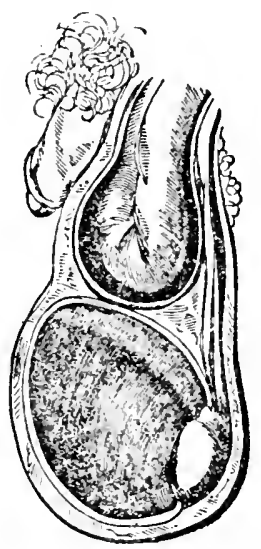

Fig. S0.-Hydrocele Complicated by Hernia.

hydrocele when it is viewed through a tube with a candle placed on the other side of the scrotum.

This sign is not infallible, however, as the thickened walls of the sae or turbidity of its contents from pus or blood prevent the light from being transmitted.

As a last resource in diagnosis a suspected hydrocele may be aspirated with a fine needle, or the patient may be prepared for operation and the contents of the scrotum exposed by an incision.

\section{TREATMENT.}

The spontaneous healing of a hydrocele in an adult is such a rare occurrence that operation is always called for. 
Puncture with a troear may be regarder as paliative only, for the sac always fills up again with fluid in a short time.

Technique.-The serotum is grasped in the hand and made trise, and after locating the position of the testicle, which is usually a little below and lying behind the sac, the trocar, with its point lirected obliquely upward and inward, is thrust into the swelling, ant the fluid is withdrawn.

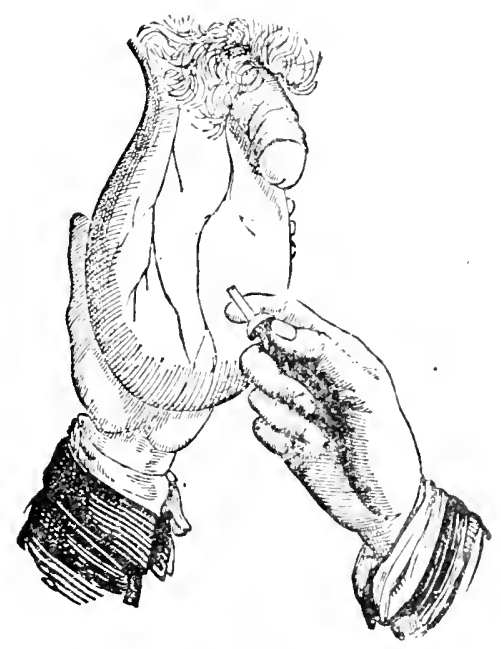

Fig. S1.-Tapping a Hýdrocele.

Radical Treatment by Injection.-This is succes: ful in many cases of hydrocele of moderate size and where the walls of the sac are not thickened, although it is more liable to be followed by a reliajec than after incision.

The advantages of injection are that no anasthetic is required and the patient is only confined to the house three or four diays.

Technique.-A hypodermic syringe is filled with 30 drops of pure carbolic acid, and the needle is introduced into the carity of the sac. The hydrocele is then tapped with a trocar in the ortinary way, and the fluid drawn off entirely. It is necessary that the sac should be completely emptied, for, if fluid is left in. the curnoli: acid is diluted, so that it does not produce the necestary amount of

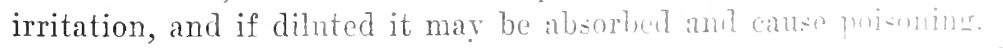

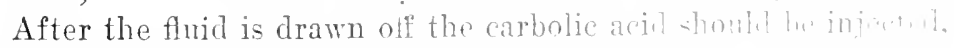
through the hypodermic needle. Which has romaimul in "th, and 
the carbolic acid is not withdrawn, but left in the sac. Inflammatory reaction is excited, but the exudate is gradually absorbed, and the sac becomes obliterated by the formation of adhesions between its risceral and parietal walls.

Incision of the sac is preferred to the treatment by injection in the following cases (Morris):-

I. When the sac is very thick, opaque, cartilaginous, or calcified.

II. If doubt exists as to whether the hydrocele is congenital or is a hydrocele of a hernial sac with a small opening into the peritoneum.

III. When a hernia complicates liydrocele and a radical cure of both is desired.

IV. When a loose or pedunculated fibrous body is present in the tunica raginalis.

$\mathrm{V}$. When organic disease of the testicle is suspected.

VI. When on account of ill health or lessened resistance the risk of inflammation after injection is especially to be dreaded.

There are two methods ${ }^{1}$ of performing the operation of incision:

(a) Vollimann's Operation of Simple Ineision.-In this procedure the sac is opened by a longitudinal incision through the scrotum, and after the fiuid is evacuated the edges of the walls of the sac are stitched to the edges of the wound in the skin, to prevent leakage into the cellular tissue of the scrotum. The cavity of the sac is packed and allowed to heal by granulation, and in this way the sac becomes obliterated. The patient is able to get out of bed in a week, and the wound is healed by about the third week.

(b) Von Bergmann's Operation of Incision and Excision of the Tunica Vaginalis.-Tillmanns considers this the best radical operation. The sac is laid bare by a longitudinal incision, and by blunt dissection is freed from the cellular tissue. The redundant portion of the sac is then cut away with scissors from the testicle, but leaving enough of the wall of the sac to cover the testicle itself. The external wound is closed with sutures, and a dressing applied which will exert compression. The wound is cicatrized in from eight to twelve dars. Relapses are more certainly prevented through excision than by other methods, and the wound heals in a very short time.

${ }^{1}$ An excellent operative procedure recently suggested by Doyen for thinwalled hrdroceles consists in ineising the sac and everting the testicle so that it lies ontside the sac. The wound in the sac is then sutured to prevent the return of the hydrocele, and the incision in the serotum is elosed without drainage. 


\section{HYDROCELE OF THE SPERMATIC CORD.}

(a) The cystic form is found as one or more smill a t a a

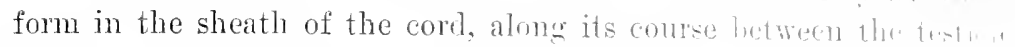
and the internal ring. The cysts are caused by an eflusion of smous fluid into some part of the processns vaginalis, which was not chliterated after the descent of the testicle.

The cysts nsually occur between the testicle and externial ling. and may extend so far down into the scrotum as to dimplane the testicle to one side; in a few anses the cyst lies within the ingminal canal.

Occasionally the cyst forming a hydrocele of the cord is not closed at either end. but conmunicates with the tunien raginalis and also the peritoneal cavity (communicating hydrocele of the cord). and it is a frequent occurrence for the ryst to communicate with the sae of the tunica raginalis testis. The cysts are shaped lilit an egg, and range in size from a pea to a hen's egg.

(b) The diffuse form of hydrocele of the cort is of extremely rare oceurrence, and consists in an welematous infiltration of the entire sheath of the cord, ceasing abruptly at the testicle below. "The chief importance of hydrocele of the cord is from a diagnostie stamipoint: in differentiating it from eryptorchism or hernia.

\section{TREATMENT.}

The use of injections of earbolic acid is attended with some danger, as the eyst may commmicate with the peritoneal cavity and the carbolic acid may flow back into the ablominal carity.

Operation is usually called for, and consists in division of the common sheath of the cord, in a longitudinal direction, ind cxinction of as much of the sac as can be separated from the cort. If the sac is foum to be open, communicating with the preritoneal carvity it

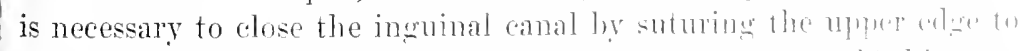
Pompart's ligament, as in bassinits ountation for hermia. If this were

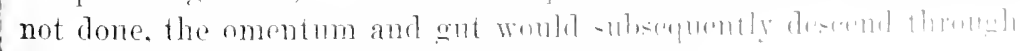
the patnlous inguinal canal, and camtec hernia. 


\section{HÆMATOCELE.}

ITrmatocele is the name given to the tumor which forms when a hamorrhage occurs $(a)$ into the sac of the tunica vaginalis (vaginal hamatocele), or (b) into the scrotal tissues around the testicle (extraraginal hrmatocele), or $(c)$ with the sheath of the spermatic cord (hamatocele of the eord).

\section{ETIOLOGY.}

A predisposing cause in the shape of disease of the walls of the tunica vaginalis or of the testicle is very apt to be present.

The cxciting cause of the hæmorrhage is always traumatism, which may be slight, such as coughing, sneezing, or straining at stool,-or it may be severe and the result of a blow or lick upon the scrotum or the accidental puncture of the testis with a trocar in tapping a hydrocele.

\section{SYMPTOMS AND COURSE.}

Swelling of the scrotum takes place very quickly after the injury, and a tumor forms, which is a hard, smooth, globular swelling surromnding the testicle. After a few days inflammatory thickening of the tissues occurs, and the hæmatocele is liable to be mistaken for a neoplasm of the testicle.

The blood-clot may remain for years without being absorbed, in which case the walls of the tunica vaginalis become thick and rense, and the testicle undergoes atrophy. On the other hand, the clot may become infected, and suppuration, ending in abscess, occurs.

\section{TREATMENT.}

In recent cases a moderate effusion of blood may be absorbed with the assistance of rest in bed, elevation of the scrotum, and 25- to 50-per-cent. ointment of ichthyol; but spontaneous absorption is of seldom occurrence, and operation is usually necessary.

The operative tratment consists in laying open the sac by a free incision and evacuating the blood-clot. The sac of the tunica raninalis may afterward be treated as a hydrocele, and either exsected or its carity packed and allowed to granulate. 


\section{HÆMATOCELE OF THE SPERMATIC CORD.}

This is a rare affection, and may occur from direct violence or from the strain of lifting a heavy weight, even though the cord is perfectly healthy.

A fusiform swelling rapidly develops along the cord, which is easily mistaken for hernia. After a few hours the swelling becomes hard and tense, with ecchymoses into the surronnding tissues, and the diagnosis is made clear.

The treatment is conducted on the same lines as for hiematocele of the tunica raginalis.

\section{VARICOCELE.}

Varicocele is an abnormal dilatation of the reins of the spermatic cord, and is analogous to the varicosities which occur in the reins of the lower extremities.

A slight varicocele often disappears spontaneonsly after marriage, when sexual intercourse is duly regulated and also in old age.

\section{ETIOLOGY.}

The causes of raricocele are, in a general way, those of varicosities elsewhere; prolonged standing, ungratified sexual excitement continued for some time, and constipation, all tend to cause passive congestion and disturbance of the circulation, with increase of hydrostatic pressure in the veins of the cord.

The spermatic veins are more liable to enlarge than others, because their valves are insufficient to uphold the long colmmn of blood, which also derive but little external support from the loose celluliar tissue of the scrotum in which they lie.

Billroth believes that there is an inherited predisposition to the formation of varicosities, which is first manifested in the spermatic veins, and later in those of the rectum and legs.

Taricocele occurs most frequently on the left side. becanse. whil. the right spermatic rein enters the rena cara at a sharp angle. the left spermatic vein, after passing underneath the sicmoid thexure, 
empties at a right angle into the left renal vein. The pressure exerted by the colon and the right-angled insertion of the vein tend to impede the current of blood, and cause an increase in the hydrostatic pressure, which is greater on the left side than on the right.

\section{SYMPTOMS.}

In robust and vigorous men varicocele of moderate size causes but little discomfort, at most only a sense of weight and dragging in the spermatic cord, after long standing, walking, riding, or any sudken exertion. These things all exaggerate the swelling of the reins, and the discomfort from them is always increased toward evening.

In young men who are weak or anæmic or who have previously masturbated excessively, a feeling of fullness and burning in the scrotum and cord, accompanied by pains radiating into the abdomen and occasionally marked neuralgia of the testicle, is often met with. Such individuals are frequently sexual hypochondriacs, and suffer from mental depression and the fear of impaired virility, or even impotence, arising from the varicocele. They should be taught to regard the varicocele, if moderate in size, with indifference, as it has a natural tendency to subside as age advances and particularly after the regulated coitus of marriage is instituted.

It is only in cases of very pronounced varicocele, where the circulation is materially impaired, that serious atrophy of the testicle occurs, althongh in every case of varicosities of the cord the circulation in the testicle is somewhat interfered with, and it is softer and smaller than normal.

The spermatic rein may become the seat of disease, and in rare cases thrombosis, phlebitis, gangrene, etc., may occur.

\section{DIAGNOSIS.}

The diagnosis of varicocele is usually easy, and the vems in the scrotum feel like a bunch of angle-worms. In very marked cases the enlarged blue reins can be seen shining through the skin of the scrotum. In cases of moderate severity the testicle is slightly flabluy and the skin of the scrotum relaxed.

If the patient lies down, the swelling disappears, not suddenly, like a hernia. but more gradually, and almost imperceptibly; and, if he stands upright again, pressure with the finger over the 


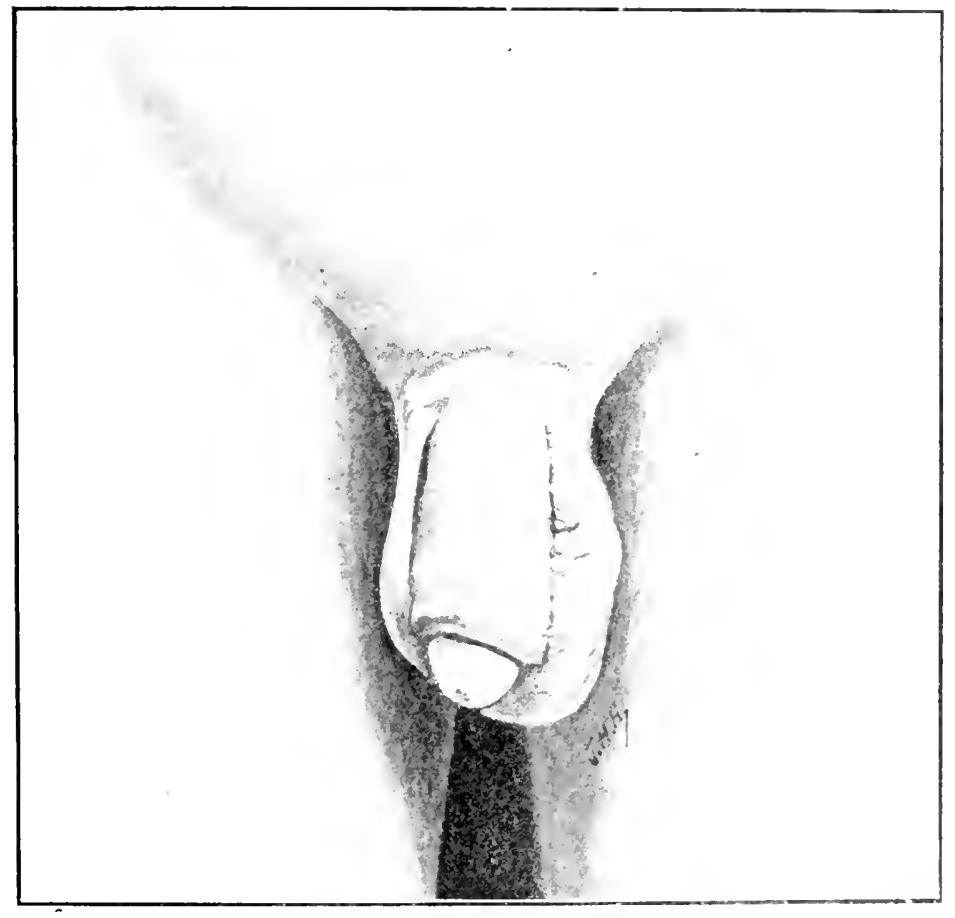

Fig. S2.-Varicocele. (Author's Case, from Kings County Hospital.) 

external ring does not prevent the veins from rofllin will blowd, whereas in hernia the omentum is provented from duscunding by pressure over the ring.

\section{TREATMENT.}

Palliative treatment is all that is reyuired in raricocele of monerate size and which causes but little discomfort. The scrotum sluould be supported with a well-fitting suspensory bandage, and, locally, will douching is of service in giving tone to the musckes. Constipation should be prevented, and the patient should aroid erotic excitement or too frequent coitus.

Operation is required only in marked cases, when the support of a suspensory bandage is not sufficient to remove the annoyance of weight and dragging, after walking or long standing, or in cases where a considerable degree of enlargement of the reins exists. Uperation is also called for, in persons desiring to enter the army, nary, or yolice service, in which any degree of varicocele is regarded as a di-ability.

The operation of subcutaneous ligation of the veins is popular with some surgeons. It has the adrantage that the time of convalescence is a little shortened, but its disadrantages are weighty. A rein is very liable to be punctured, and an hematocele occurs. The silk ligature remains permanently, becomes imbedded in scar-tissue, and sometimes causes persistent neuralgia, making it necessary to open up the wound and exsect the knot, or the knot may slip and the rein is not occluded. The danger of tying in the spermatic cord exists. but can be guarded against by due care in holding the cord out of the war.

Relapses occur with greater frequency after subcutaneous ligation than after excision.

Excision of a portion of the veins is the operation to be preferret, and the patient is only kept in bed for a week or ten days. By the open operation it is possible to aroid all the dangers attendant mpon the subcutaneous ligation.

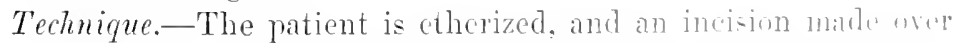
the bunch of reins, begimning near the external ingminal rimer and extending two inches down over the scrotum. The reins are exposed hy dissection and ligated in two places, about an inch apart. The portion of the reins lying between the ligatures is then cut out with scissors, and the cut ends of each portion of the win are hrought together into apposition and held so, by tying the ends of the liga- 
tures together, and in this way the rein is shortened. The wound is then closed by sutures.

The pratient is kept in bed for a week, and on a sofa for another weck, and after the third week is completed he can begin his active labors, and the same length of time should elapse after the subcutaneurs ligation.

After either operation a hard mass remains in the scrotum, composed of coagula in the reins and infiltration around them. This is alsorbed and disappears in from two to four months. .

Atrophy of the testicle exceptionally follows either operation, even when the cord is not included in the ligature. 


\section{CHANCROID AND ITS COMI- PLICATIONS.}

\section{CHAPTER XIX.}

\section{CHANCROID.}

Synonyms.-Soft chancre, or simple venereal ulcer.

\section{ETIOLOGY.}

There are at present two views held in regard to the etiology of chancroid: (a) It is supposed by some to be due to a particular microorganism produced in another chancroid, which is named, after its discoverer, the bacillus of Ducrey. (b) The other view, which is more

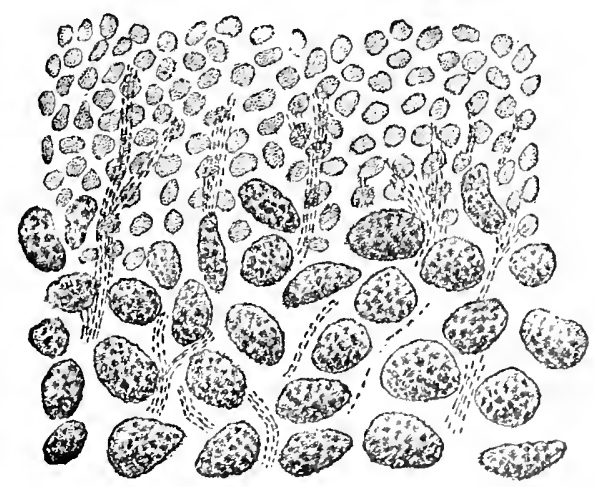

Fig. 83.-Section of Chancroid, showing Ducrey's Bacillus, which has the form of small rods arranged in chains.

generally held, is that chancroid is merely an active form of woundinfection, and that it is caused by the inoculation nnder the shin of any of the pus-producing micro-organisms. and in this and of here respects resembles chinically impetigo contagiosa or cuthyma. 


\section{MODES OF CONTAGION.}

(a) Immentinte: i.e., through direct contact, as in coitus, or by digital examination, etc.

(b) Mediale, in which contact is not made with the chancroid, but some of the pris is deposited upon an instrument, towel, chamber utensil, water-closet scat, etc., and from this point is brought into contict with another person. who becomes infected.

The pus from a chancroid is acrid and irritating, and has the property of softening and corroding the healthy epidermis; so that an abraded surface is not essential for the entrance of the microorwanisms inder the skin.

\section{FREQUENCY OF CHANCROID.}

Chancroid is more common among the lower classes than among cleanly and well-nourished people, as its development is farored by filthy halits and dirty surroundings, and all the accompaniments of prisation and misery.

\section{COURSE.}

Chancroid is usually multiple, although it sometimes exists singly. The fact of the sores being multiple is accounted for in the following ways:-

(a) A number of points are inoculated with chancroidal pus at the time of contagion.

(b) Through autoinoculation.

Autoinoculation may be defined as the action of pus formed in one chaneroid producing other chancroids in the same individual: i.e., the inoculation of the patient with pus from his own chancroid.

After inoculation, in about twenty-four hours several pustules ajpear. which spread at the margins; the tissnes adjacent are involved and break dorm, and the nleeration extends.

\section{CHARACTERISTICS.}

In chaneroid there is no period of incubation, and it is generally noticent on the third day after coitus.

The location is merally on the genitals, although it exceptionally ocemrs nut the fingers.

In shape the sore is usually irregular, although it may be round or oral.

Number.-It is cxceptional for chancroids to exist singly. They 


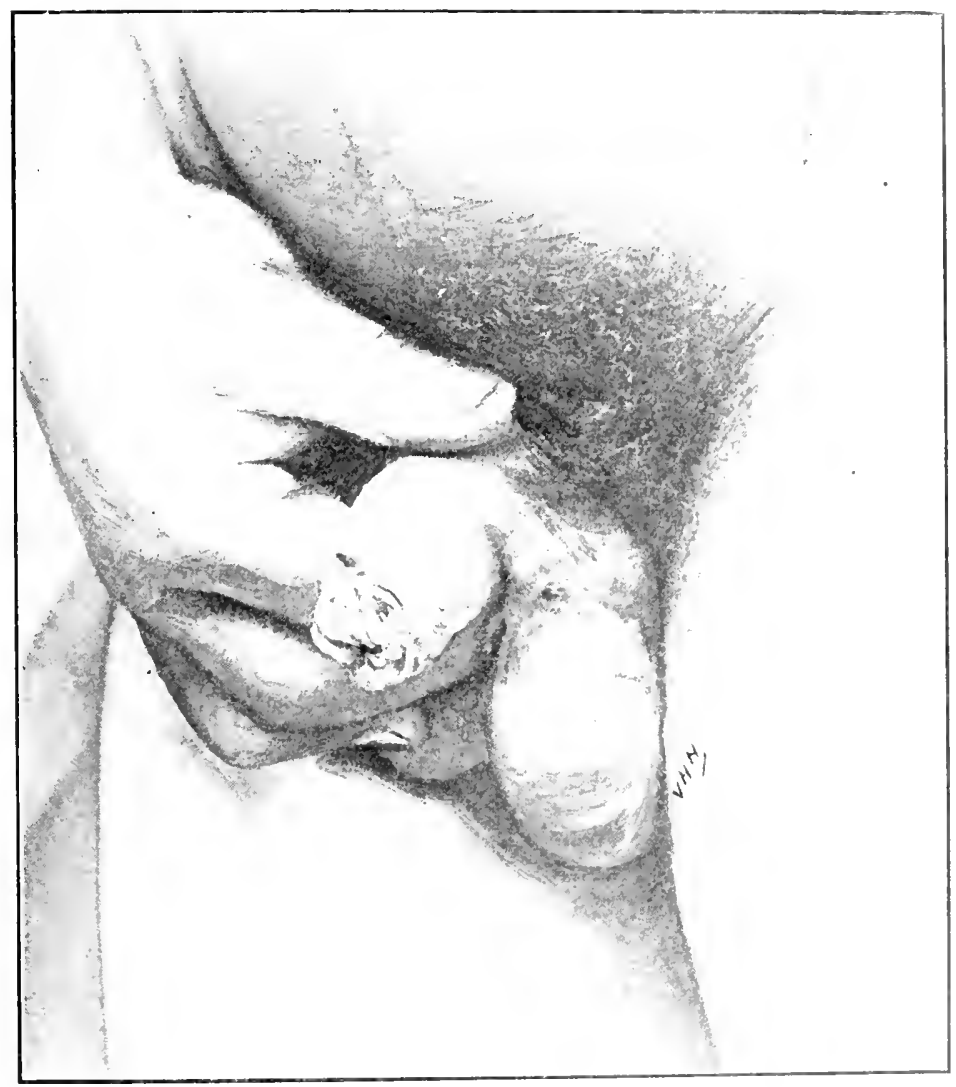

Fig. S1-Chancroids of the Prepure. Anthor's (ase from Kings County Huspital.) 

are usually multiple, either from the simultaneons inoculation of several points at first or from subsequent autoinoculation.

Pain is usually present to a considerable extent.

The base of the sore in a typical case is deroid of indurulium. but if an excessive amount of inflammation has taken place-as a result of insufficient and frequent cauterization, prolonged contact with irritating urine, pus, or acrid secretions-an inflammatory or boggy liardness is often present. This does not feel like the induration of a chancre, which is sharply defined and which resembles a piece of cartilage moder the skin, but is doughy or boggy in feching, and shades off gradually into the surrounding tissues.

The floor of the chancroid is rough and meren, and covered with a dirty-grayish deposit.

The edges of the sore are undermined, and the discharge is abundant, purulent, and sometimes bloody.

Duration of the chancroid may be divided into stages:-

I. Progressive stage, during which each sore extends by a breaking down and ulceration of the edges, and fresh sores are continually forming as a result of autoinoculation.

II. Stationary stage. After a time the pus loses its virulence, autoinoculation of fresh surfaces no longer occurs, and the ulcers cease to increase in size, but remain stationary.

III. The reparative stage begins when the sores are covered with healthy granulations and cicatrization is in progress.

The various stages require about six weeks in time for uncomplicated cases, but relapses from autoinoculation are very freprent, and delay the healing process.

Microscopic examination of a chancroid shows it to be composed of a small round-celled infiltration which takes place in the skin and subcutaneous tissnes, and which leads in time to coagulation-necrosis.

The lymphatics are not involved, but remain open and gaping, ready to convey micro-organisms or toxins to the nearest limphatie gland, and in this way we can account for the frequent occurrence of bubo.

Until 1852, chancroid was regarded as a syphilitic manifutation; yet the true difference between the two diseases would have been sooner discovered but for the mixed sore. It sometimes happens that an individual is syphilitic and has accidentally aequired chancroids.

As a result of contact with both poisons, usmally during coitus, another person receives a double infection: i.e., the rirus of syphilis 
and that of chancroid are both inoculated at the same moment. The chaneroid appears in twenty-four lours, but, on account of the longer inculation of sylhilis, the chancre is not erident for at least three weeks. Ifter this period has gone by, the base of the chancroid becomes hard and selerosed and the sore is transformed into a true chancre which is followed by symptoms of constitutional syphilis.

\section{DIAGNOSIS.}

It is often difficult to make a differential diagnosis between chancre and chancroid, particularly in the cases where an excessive amount of inflammation has occurred and a condition of boggy inflammatory induration is present. We should always bear in mind the possibility of a mixed sore being present, and it is usually well to allow three or four weeks to elapse before excluding syphilitic infection.

The principal points to consider in making a differential diagnosis are as follow:-

I. The period of incubation: i.e., the time which elapses between coitus and the appearance of the sore.

II. The absence of the cartilaginous induration, which characterizes the primary lesion of syphilis.

III. The ragged, punched-ont appearance of the sores, which are usually multiple.

IT. The character of the accompanying enlargement of the inguinal lymphatic glands.

Herpes præputialis is sometimes incorrectly diagnosed as chancroid. This mistake ought not to oceur, if due care is used in the examination. Herpes appears as a number of small vesicles, which form upon the glans, prepnce, or skin of the penis. The resicles soon break, and leare small, round. superficial erosions, which rapidly heal under a simple dusting-powder.

\section{TREATMENT.}

(a) Abortive. - If the chancroid is seen early enough, the indication for treatment is to destroy the chancroidal character of the sore and transform it into a simple non-infected ulcer.

Wethor.s.-Nitrate of silver should never be used for this purpose, as its cautcrizing action does not penetrate deeply enough into the sulbetauce of the sore.

The bet means to this end is the Paqnelin cautery. Fuming 
nitric acid or the acid nitrate of mercury applied to the sore upon a glass rod will answer the same purpose. The ulcerating surtares should be washed clean and anæsthetized by chloride of ethyl, a drop of carbolic acid, or cocaine solution before canterizing.

It is an error to canterize every chancroid as a routine measure, and we can lay it down as a rule nerer to cauterize unless the chancroil is free from complications, as follows:-

Contra-indications for Cauteriation.-(a) If the inflammation is already excessive and much inflammatory adema is present, the irritation of cauterizing will increase it.

(b) If inguinal adenitis is present, the bubo will be made worse by cauterizing the chancroid.

(c) In the case of a healing cicatrizing chancroid the chancroidal virus has already disappeared, and canterization is nseless.

(d) If a number of chancroids are present, and only a few are exposed, the entire number should be destroyed, or none at all, for if any are left untouched, they will secrete pus, which will infect fresh surfaces.

(e) If a chancroid of the meatus or in the urethra is canterized, the resulting cicatricial contraction after the sore is healed will produce too much deformity of the parts affected.

(b) The methodic treatment is carried out by means of:-

I. Cleanliness.

II. Antisepsis.

III. Rest.

The chancroids should be kept free from the accumulation of discharge by means of frequent washing with solutions of bichloride of mercury, 1 in 5000 or 10,000; or carbolic acid, 2 per cent.

Dry dressings or dusting-powders should only be need under the foreskin and never upon a sore located on the integument. The powder, which is exposed to the air, dries, sticks fast, and tears open the wound when the dressing is changed.

The following antiseptic powders are the most servicealble: Iodnform, salicylic acid packed into the sore, aristnl, subsallate of hisuruth (dermatol), resorcin, and nosophen.

When the chancroid is located upon the integument, wet dresings are called for. A piece of cotton or ganze should be wet with bichloride, carbolic, or 'Thiersch's solution. coverent with a piece of guttapercha tissue to prevent evaporation, and held in place with a bandate. 


\section{COMPLICATIONS OF CHANCROID.}

I'lic accompanying inflammation may at times become excessive from such generul canses as debility or some dyscrasia, or local causes, such as contact of urine or lack of cleanliness. An excessive inflammation, if untreated, is apt to terminate in gangrene and sloughing of the jarts.

Subpreputial Chancroids Complicated by Phimosis. - When chancroids are located under a long and tight foreskin, the resultant swelling and nedema prevent its retraction, and render the diagnosis difficult. Artificial inoculation of the patient upon the thigh, with pus from under the foreskin, has been resorted to, to differentiate between chancre and chancroid.

The possibility of the presence of a mixed sore should always be borne in mind in these cases.

TREATMENT.

The preputial sac may be kept clean by the use of antiseptic injections with a flat-billed syringe under the foreskin, using a weak carbolic or sublimate solution every hour or two. An attempt should

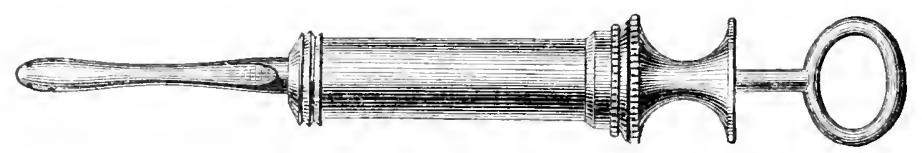

Fig. S5.-Taylor's Flat-Billed Syringe for Washing Out the Balano-preputial sac.

also be made to retract the foreskin and canterize the sores, which can be facilitated by prolonged soaking of the penis in hot water.

On account of the danger of paraphimosis, the foreskin should not be left retracted, but drawn back into place.

In case the swelling increases and surgical interference is not resonted to, the dorsum of the prepuce becomes gangrenous and sloughs in part, or the entire foreshin sloughs off (spontaneous circum(cision), or the glans penis becomes gangrenous and sloughs, either entirely or partially.

If gangrene is threatening, recourse must be had at once to operative measures. The prepuce should he slit up, upon the dorsum, with a curreal bistoury, to relieve the tension. 
The chancroids are exposed and cauterized, and in ardition the entire surface of the fresh cut wound is cauterizer, as wcll, with either the Paquelin cautery or nitric acid.

Circumcision should nerer be performed in the presence of chancroids on account of the danger of infecting the wound with chancroidal virus, and converting its entire surface into a chancroidal ulcer.

In all cases of subpreputial chancroids, if at all severe. the radical treatment of slitting up the foreskin and cauterizing is to be preferred to temporizing with injections under the foreskin.

When chancroid of the frcmum exists, the artery is liable to be ruptured during erection and hæmorrhage occurs. To prevent this accident, it is proper to tie a double ligature around the franum, divide the frænum between the ligatures with a pair of scissors, and canterize the sore.

Paraphimosis is the condition in which a long foreskin is retracter? behind the glans, and, from the swelling and odema which take place

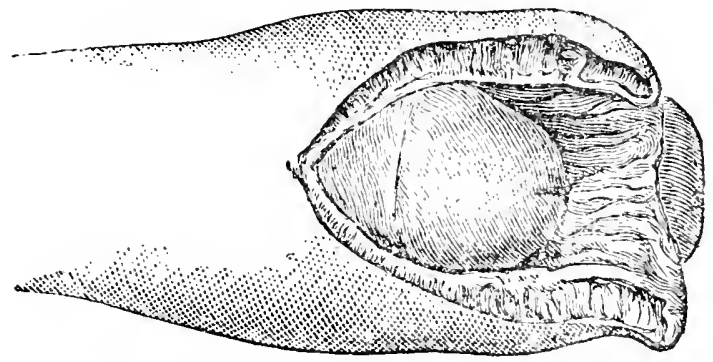

Fig. 86.-Dorsal Incision through Prepuce to expose Chancroids.

in it, is prevented from being drawn back into place. Gangrene and sloughing may result, if the condition is left untreated. The constricting band should be cut upon the dorsum by incerting a curved bistoury under it and cutting outward.

\section{PHAGED正NA.}

This, most formidable of all the complications of (hanemir. is rarely encountered at the present day.

It is due to debility of the individual attacked, as a result of some dyscrasia, alcoholism, syphilis, tuberculosis, privation, ete. 
Two varicties are noted:-

(a) Serpiginous, in which the ulceration creeps slowly along, adrancing at one side. It is a very chronic condition, and may last for montlis.

(b) Sloughing, in which the parts die en masse in a short time.

\section{TREATMENT.}

The first indication is to treat the dyscrasia, by means of tonics, of which the potassio-tartrate of iron in 5-grain doses every three hours is highly spoken of, quinine in tonic doses, and opium for its effect on the pain. Easily-digested nutritions food and milk-punch should be administered.

Locally the ulcerating surface should be cauterized, with the Paquelin or nitric acid, and dressed with iodoform. In cases which fail to respond to the ordinary treatment hot baths are valuable. The body is immersed for days and weeks at a time in a tub in which the water is liept constantly changing and at the same temperature. Under the use of the hot water the sloughing ceases and the ulcers take on healthy granulation.

\section{BUB0.}

The term bubo is applied to the inflammatory enlargement of any lymphatic gland, but especially in the inguinal region.

It accompanies chancroid in from 30 to 50 per cent. of all cases, and is particularly liable to occur in debilitated badly-nomrished subjects.

\section{ETIOLOGY.}

There are two views held in regard to the causation of bubn:-

(a) It is believed that the inflammation of the lymphatic glands is canced by the micro-organisms from the chancroid, which are carried to the glands through the lymphatic ressels and deposited in them.

(b) It is held by others that the micro-organisms themselves are not carried to the glands, but only certain chemical substances (toxins), which are formed in the chancroid as a result of bacterial growth and deveited in the adjacent lymphatic glands. 


\section{COURSE.}

One gland alone may be affected, but, as a rule, the whote chain on one side is involred, and it is not infrequent for sereral ghands on both sides to be attacked.

Exceptionally the involrement may disappear by resolution, but in the great majority of cases the glands suppurate and break down.

The process of softening of the chain of glands, and their breaking down and suppuration. and the slow evachation of the pus thrown a small opening, is a rery protracted one, and may last for months unless the diseased glands are completely extirpated by surgical measures.

The accompanying table indicates the chief points of difference between the glandular enlargement of syphilis and that of chancroid:-

\section{DIFFERENTIAL DIAGNOSIS OF BUBO AND SYPHILITIC ADENOPATHY.}

\section{CirATCROID.}

One side affected, as a rule.

\section{Shape:}

Irregular and boggy.

$$
\text { Sive: }
$$

Large.

$$
\text { Nomber: }
$$

May be single.

General Characteristies:

Considerable amount of inflammation, causing adherence to overlying skin. Generally suppurate.
SipHILIs.

Both sides affected.

Shrtye:

Tiegular, smooth, and hard.

sise:

Small.

Number:

Always multiple and arranged in a chain.

General Characteristics:

No inflammation. Not adberent to skin, but freely morable. Do not suppurate.

\section{TREATMENT.}

The indications are:-

I. To prevent suppuration.

II. After suppuration has taken place to evacuate the pus and extirpate the diseased glandular struetures.

To prevent suppuration, the most important measure is rest in bed, supplemented by the pressure of a sind-hat orer the inflamed gland.

Cold, in the form of an ice-bag or evapnrating lotion, is sometimeof use. Tincture of iodine is often prescribut, but is of little use in 
nssisting resolution; if ordcred at all, it should not be painted over the inflaned gland, but in a ring around it.

Ichthrol ointment, 50 per cent., is frequently used, but is of less valne here than in other conditions.

The jujection of antiseptic solutions into the substance of the gland with a hypodermic syringe, using carbolic acid (15 per cent.) or henzuate of mercury ( 1 per cent.) is a rational measure, and has attained a fair degree of success in preventing suppuration.

The various measures above mentioned, howerer, rarely succeed in preventing the formation of pus, and on that account it is better not to lose valuable time, in efforts to abort the suppuration, which will most likely prove marailing in the end, but to have recourse promptly to surgical measures.

Operative Treatment. - (a) Before Suppuration. - If the case comes to operation before the glands have begun to suppurate, a curvel incision should be made through the skin, and the flap turned back, exposing the entire chain of glands, which can be dissected out. If the operation is done before the formation of pus, the wound. may be entirely closed by sutures, and primary union usually occurs.

(b) After Suppuration.-When the glands have begun to suppurate it is no longer possible to close the wound entirely by suture, on accomnt of the certainty of infection, and, instead of the patient being able to walk abont in a week with the wound closed by primary mion, lie has to wait from four to six weeks for the slow healing by granulation of the open wound.

The indications for treatment after suppuration has occurred are to evacuate the pus, by a free incision, and at the same time remove completely all portions of the glandular structure, by means of careful dissection or the sharp curette. The wound is left open and packed with iodoform ganze, and heals by granulation. An objection to extirpating the glands before they have entirely broken down is that, in occasional rare instances, a permanent œdema-resembling elephantiasis of the penis, scrotum, and inguinal region follows, in consenmence of the obliteration of the lymphatic ressels in the process of wound-healing. ${ }^{1}$ For this reason Krulle adrises the applications of hot fomentations till the gland is entirely broken down, when the jus is exacuated through a small incision. Every second day the

(hul of orer a hmmaer cases of bubo treated by dissecting out the glands, the anther has only en this complication oceur once. 
pus should be squeezed out and the carity of the wound wa-hed with 1-per-cent. nitrate-of-silver solution. Lnder this treatment the patient can walk about, and aroids the necessity of lying in bed. This plan may work successfully when the glands break down rapidly, bu in many instances the suppuration goes on very slowly, and it is hettr. to make a free incision, eracuate the pus, and dissect or curette out the partially broken down remains of the glands. 


\section{SYPHILIS AND ITS LESIONS.}

\section{CHAPTER XX.}

\section{CHANCRE.}

Synonyms.-Initial lesion of syphilis; initial sclerosis; hard chancre.

Definition.-Chancre may be defined as "the first manifestation of the syphilitic poison at the seat of its entrance into the body."

'The poison of syphilis, which is inoculated into an individual and causes the chancre, is derived from the secretions and broken-down detritus formed in:-

(a) A chancre in another person.

(b) Mucous patches.

(c) Condylomata.

(d) Blood.

(e) Lrmph.

(f) It was formerly thought that the physiological secretions did not contain the syphilitic virus, but recent investigations have shown that in exceptional cases they do contain the poison.

\section{COURSE.}

When the poison of syphilis is first inoculated under the skin, it is too small in quantity to produce any symptoms. But the poison increases in amount, and, after three or four weeks have passed, the quantity is so great at the point of inoculation that the tissues react, and the chancre appears.

At this time the syphilitic virus is not distributed all through the body. lut is localized to the tissues around the chancre and in the nearest lymphatic glands. Later on in the disease the poison increases to an enormous extent, makes its way through the lymphatic system into the circulation, and the blood and lymph become surcharged with it.

The pus from a chancre, unlike that formed in a chancroid, has not the property of destroying the epidermis, and in order to produce a chancre it is necessary that the poison from a syphilitic lesion be introdued through an abrated surface. 
This fact explains the cases in which a plyysician atumls a syphilitic woman in confinement or a man has coitus with a wonan who is affected with condylomata or a chanere, and, if an abrasion did nut exist at the time of contact, inoculation does not oecur.

Every case of syphilis contracted after birth must have a chancre for its point of departure.

There is, however, one exception to this rule. A healthy woman who has connection with a syphilitic man may eseape inoculation with syphilis, but become pregnant. The child which is generated ]y a syphilitic father is infected with syphilis, and as it develops in viero the syphilitic virus passes from the child to the mother through the placental circulation, and in turn the mother beeomes infected with syphilis from her own child. In this case chancre does not form either in the mother or child.

The form of infection of the mother just described, from carrying her own child in utero, is called "Choc en retour", or "syphilis by conception."

\section{TRANSMISSION OF CONTAGION.}

The methods by which the contagion is transmitted may be classified as:-

(a) Direet.

(b) Indirect, or mediate.

(c) Inheritance.

(d) Choc en retour, or syphilis by conception.

By direct contagion we understand those methods in which the syphilis is inoculated by personal contact, snch as coitus, kissing, surgical operations, a syphilitic child infecting its wet-nurse, or a healthy child acquiring syphilis by nursing from a syphilitic wet-nurse.

In indirect, or mediate, contagion the disease is conveperl throngh the medium of some article which has been infected with the virus of syphilis, such as a spoon, pipe, eup, or cigar used hy a person with mucous patches in the mouth; an instrument used for tattouing, dental or surgical operations, which had leen used on a syphilitic individual and not cleaned afterward, or by means of underething or a bathing-suit which was stained with secretions from syphilitic lesions.

Vaccination chancre in former years was not uncommon, and was caused by vaceinating with an instrument which hikl previonsly he n used to raccinate a syphilitic individual. and whith was not afterwall cleansed, but was used still contaminated with the syphilitio virus. 
Chancres were also produced by vaccinating, with dried lymphcruts, which had heen taken from vaccine-resicles produced in an individual who was also affected with syphilis at the same time.

At the present day animal vaccine-lymph, taken from the calf, is used exchusively, and, as cattle are immune from syphilis, a chancre as the result of vaccination never occurs.

The location of the chancre depends, of course, upon the point at which the syphilitic virus is inoculated. The chancre appears most frequently on the genitals, but may be on the lips, tongue, breast, or fingers. It begins as a papule or small tumor, which increases in size; the surface becomes eroded, or ulcerated, and furnishes a secretion which is not autoinoculable. The most characteristic feature of the chancre is induration of the base, which is caused by a deposit of small round cells in the tissues underlying the chancre, and also by inflammatory changes in the blood-ressels.

\section{PATHOLOGY.}

On microscopic examination of a chancre the findings are as follow:-

I. A small round-celled infiltration of the skin and subcutaneous tissues, such as occurs in every inflammation. The infiltration begins in the blood-vessels and spreads outward toward the periphery. For this reason the form of the induration depends upon the course of the blood-ressels. When they run horizontally and near the surface, a thin, flat layer of infiltration occurs under the skin, which is called parchment induration. On the other hand, when the blood-ressels dip down deeply into the tissues, the induration is extensive and deep, and is called Hunterian induration.

II. Changes in the Blood-vessels. - The reins and arteries are both affected by the endarteritis, but the changes are more marked in the arteries. The endothelial cells of their inner coats are swollen and the lumen of the ressel is diminished. The middle coat is usually slightly thickened and infiltrated, but the important change is in the outer coat, which is the scat of an infiltration with small round cells.

In consequence of all the changes described the circulation of lilood is shut off, and coagnlation-necrosis, with sloughing of small areas of tiscue supplied ly the affected ressels, takes place.

It is duiralle to understand clearly the pathological changes, 


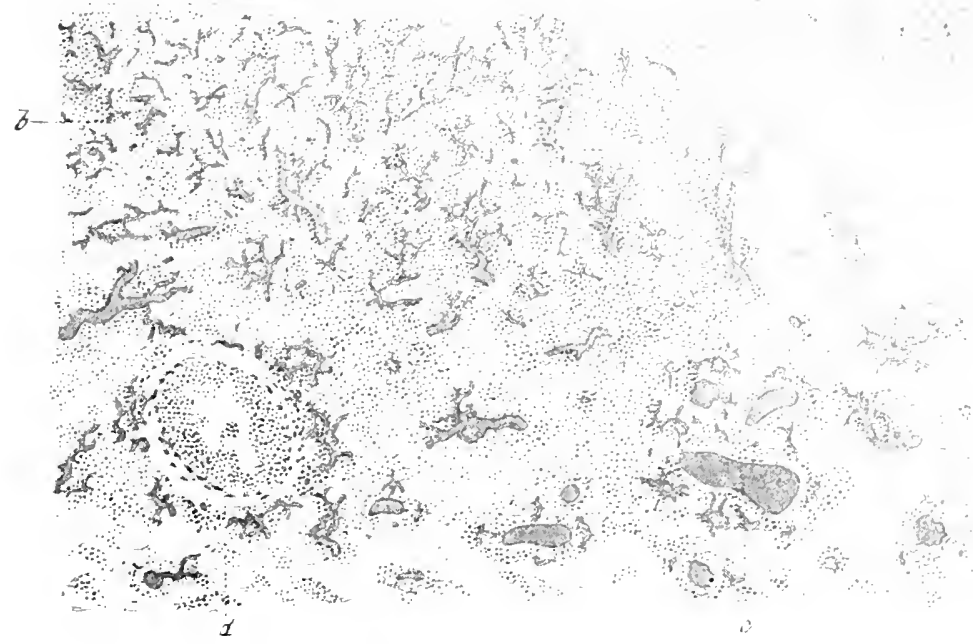

V. Section of a Chancre (Injected).

$a$, Rete mucosum.

$b$, Small round-celled infiltration with numerous injected blood-vessels

$c$, Blood-vessels with endo- and peri-arteritis.

d. Lymphatics with adventitial and endovascular changes.

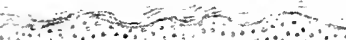

$Q$

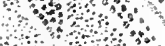

A

西

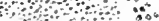

10

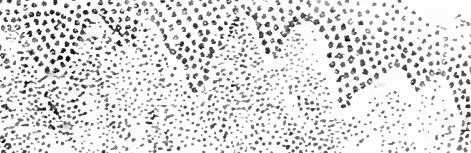

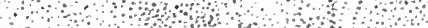

mon

and

sto

政

c

ind

4

YI. section thomeh a Papmlar Syphitite

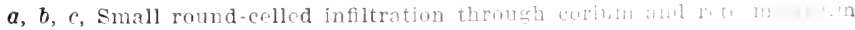

and around bair-follicles and swat-glima.

d, Blood-vessel, with infiltration of the adventutit.

c, Normal cutis. 

since all syphilitic lesions-including chancre, papule, and cummaare identical, and are due primarity to endarteritis, and, scunlarity, to the accompanying small round-colled infiltration.

The lymphatics, as a rule, are not affected, but remain open and serve to carry the virus, to be deposited in the neighboring gland.

In rare instances an endo-lymphangitis occurs, which obstructs the flow of lymph, and occasions a hard, boggy condition of the tisitus, causing the so-called indurative or scterotic adema.

The hardness and sclerosis of chancre are not entirely accounted for by the microscopic findings, because the infiltrating cells are present in spots where no trace of hardness is perceptible to the touch.

Unna attempts to account for the hardness by supposing that a deposit of colloid material takes place around and between the bands of round-celled infiltration in the older parts, but that it is not formed as rapidly as the infiltration adrances at the periphery.

The essential characteristics are always the same. but chaneres rary considerably in outward appearances, as, for example:-

(a) In the amount of induration.

(b) In the depth of ulceration.

(c) In the amount of inflammation of the surrounding tissues.

Therefore chancre is grouped into several varieties.

\section{VARIETIES.}

I. Dry papule, which is made up of a very slight amount of induration.

II. Hunterian chancre. In this variety, which is named after John Hunter, a considerable amount of induration is present. Its central part is the seat of a coagulation-necrosis, from the cndarteritis which occludes the blood-ressels and causes a depresion in the centre. which is fumnel-shaped, or like the crater of a rolano. The Jinterian chancre is the form most commonly met with.

III. Parchment chancre. This form is due to a superficial layer of infiltration lying immediately under the skin and widely sprait out in the tissues. It feels like a piece of parehment on being pinched up between the fingers.

IV. Indurative or sclerotic adema. This condition is protucent by a combination of the usual endarteritis and small pomb-celled infiltration, and in addition it is accompanied by ans inflummation of the lymphatic ressels or endu-lymphangitis. 
The circulation of the lymph is interfered with, and it transudes from the lymphatic ressels into the tissues. This combination of lathological changes gives rise to a boggy condition of the tissues, which is harder and more pronomed than in simple odema, and, on being pinched between a finger and thumb, a condition of fibrosis is felt. Indurative endema is a rare condition, but oceurs more frequently on the tumale genitals than in men.

\section{CHANCRE HEALS WITHOUT IEAVING A SCAR.}

The ulceration and necrosis of the chancre take place at the expense of the newly-formed tissue-elements rather than the normal structures of the parts affected, and consequently no scar results. Chancroids always leave a scar after healing, because the ulceration spreads and destroys normal fixed cells of the part.

\section{DURATION OF THE CHANCRE.}

A chancre may heal in a few days or may remain unhealed for many months, especially if it is located under the prepuce, and prorided no mercurial treatment is given.

\section{CHANCRE OF THE URETHRA.}

Chancre located within the urethra is a rare condition, but it does occur at times. It is usually located from one-half to one inch from the meatus, and the only symptom which it occasions is a thin discharge from the urethra, which may be easily mistaken for a gonorrhma. Examination, howerer, shows a hard, sclerotic mass surrounding the urethra under the skin, and the nearest lymphatic glands are also enlarged.

A knowletge of the fact that a chancre may be concealed within the urethra will sometimes be of service in explaining the cases of sylhilis in which the patient admits having had a slight gonorrhœa, but who denies that he ever had a chancre. In these instances the discharefe from the chancre, issuing from the meatus, was mistaken for a urethritis.

\section{DIAGNOSIS.}

The shomld always aroid haste, in making a diagnosis of chancre, num, it any dould wists, we should await the development ef secoudary symetoms. 
In the case of a sore occurring a few days after coitus it is uften impossible to exclude syphilis until at least three weeks have (1aped, on account of the possibility of a mixed infection having occurred.

When in doubt as to the diagnosis, we can wait for seconrlary symptoms-i.e., eruption, mucons patches, alopecia, fever, and hearache-to confirm it, or we can make the diagnosis by confrontation.

Diagnosis by confrontation is made by examining the individual from whom the patient acquired the sore, in order to determine the presence or absence of syphilis.

The most reliable diagnostic points of chancre may be summarized as follows:-

I. Indolent painless swelling of the nearest lymphatic glands, which are polyganglionic: i.e., arranged in a chain.

II. Induration of the base of the sore, which consists of a hard, cartilaginous induration, and feels like a foreign body imbedded in the tissues.

III. History of the period of incubation, lasting about three weeks.

\section{DIFFERENTIAI DIAGNOSIS BETWEEN CHANCRE AND CHANCROID.}

\section{Chatcre.}

Incubation:

Three weeks.

\section{Commencement:}

Begins as erosion or papule, which undergoes superficial ulceration.

\section{Numbers:}

Single generally. If multiple, so from beginning.

\section{Edges:}

Level or sloping and adherent, giving "seooped-out appearance."

\section{Floor:}

Smooth, shining, red, or raw; cortred with slight deposit.

\section{Seeretion:}

Scanty ; slightly purulent; not autoinoculable.

\section{Progress:}

Slow.
CHANCROID.

Inculution:

Kone.

\section{Commenement :}

Pustule or ulcer, and remains so.

\section{Tumbers:}

Multiple from beginning or became so by autoinoculation.

\section{Edges:}

Abrupt and undermined.

\section{$F(1, n):$}

Rough; worm-eaten: "Wash luttler" in appearance.

\section{Sifretion:}

Abundant and furrulent: autimeuIable.

$$
\text { Pron: }
$$

Riipid. 
Cilancre.

Induration:

Constantly present.

pain:

Aloent.

litbo:

Constantly prent. (See table in section on "liubo.")

sicar:

Ileals withont scar.
Chaxcroid.

\section{Induration:}

None or boggy.

Pain:

Present.

\section{Bubo:}

Oceurs in 1 out of 3 eases.

Sear:

Sear always remains.

\section{PROGNOSIS.}

It may be stated, in a general way, that the sererity of the chancre bears some relation to the gravity of the secondary manifestations. When the chancre is benign, it is probable that the secondary lesions will be superficial and of a mild type, which can be explained in the following way: In a debilitated individual the tissues react more violently to the syphilitic poison, and consequently both chancre and semularies are more severe, while, on the other hand, the tissues of a vigoroms, well-nourished person are in a better condition to resist the action of the poison.

The trpe of chancre, however, is no indication as to the severity of tertiary lesions, and the severest tertiary aecidents often follow a mild chanere.

This is sometimes, no doubt, due to the fact that treatment is often neglected or insufficient in mild cases.

\section{TREATMENT OF CHANCRE.}

The cardinal rule in the treatment of chancre is to avoid every form of irrilation; causties, strong applications, or mechanieal irritunts shonld never be nsed.

When the chancre is located on the integument, a moist dressing shontil be used, consisting of a piece of cotton or ganze, soaked in bichlorite solution ( 1 in 2000 ). extract of hamamelis, Jisterine, Thiersch: fluid, or black wash, and covered with a piece of guttapereha tissue to prevent eraporation.

If the chancre is located beneath the prepuce, a simple dustingpowter of calmmel, dermatol, iodoform. or aristol, which is covered with cotton. forms a suitable dressing that absorbs the discharge and fuevents the open sore from being infected with pus-organisms. 
Mercurial plaster is a useful application to the chancre in any location, and the local contact of the mercury with the chancre induces a certain amount of absorption.

\section{ABORTION OF SYPHILIS AFTER INFECTION.}

It is commonly held to-day among syphilographers that it is better to avoid giving mercury internally until the secondary symptoms of syphilis appear. It is impossible to abort the syphilis by the premature use of mercury, and the appearance of the eruption is only retarded.

It is believed by Ehrmann and others that the patient is rendered more liable to tertiary affections if mercury is given before the eruption appears, and, while it is true that its internal administration causes the rapid absorption and disappearance of the chanere, if used too early in the disease, it is detrimental, in the long run, to the patient.

The early excision of the chancre, before the appearance of the secondary simptoms, was widely recommended and practiced a few years ago, upon the ground that the virus was strictly localized to the chancre and the tissues in its immediate ricinity, and that the poison might be entirely remored from the hody by excising the sore. A more extended experience has shown this to be fallacious, and tn-day the procedure is regarded as useless. 


\section{CHAPTER XXI.}

\section{SYPHILIS.}

Srphilis may be defined as a chronic infectious disease, due to a succific poison, probably a micro-organism. Its local manifestations are, primarily, inflammatory changes, beginning in the coats of the lolood-rcssels and inrolring the perivascular tissues. After two or three yours its contagions element disappears, and the discase assumes the nature of a diathesis.

\section{THE VIRUS.}

The virus, or poison, of syphilis is, in all probability, a microorganism belonging to the class of schistomyceten, although it has not yet been demonstrated with certainty. The proof tests, which bacteriologists insist upon, of isolation of the bacillus, its cultivation, and the subsequent production of the parent-disease by inoculation of the cultivated organism into a healthy animal, have not as yet verified the existence of the specific organism.

\section{CARRIERS OF THE POISON.}

The virus of syphilis is contained in the secretions, which are derived from the lesions of the primary and secondary stages, and in the blood, lymph, and semen.

Recent investigations have also shown that the physiological secretions of glands-i.e., the milk, tears, and salira-do, at times, but fuly exceptionally, contain the poison. It is of frequent occurrence, howerer, for the saliva and milk to be contaminated or mixed with the discharges from mucous patches from the mouth or nipple.

Syphilis loses its contagious quality in the tertiary stage, and the hlood and secretions from lesions are no longer capable of conveying the disensec.

\section{ANALOGY BETWEEN SYPHILIS AND THE ERUPTIVE FEVERS.}

A listinct parallel, which has becn pointed out by Jonathan Irutchinson, cxists in the course of syphilis and small-pox, measles, -witina. Ate. All these discascs are due to a special and distinct $(0,9)$ 
treatment relapses ccase after from one to three years. But it does not necusarily follow that the syphilis is at an end because a consilerable length of time passes without a relapse. After a period of lateney, which may extend over many years, new symptoms may again derelop.

The late lesions differ from the early ones in that they are of a grave natme and do not tend to spontaneous recovery, but are more aj)t to destroy the organs attacked. The secretion which they furnish has also lost its contagions properties.

\section{MODE OF INCREASE OF THE VIRUS.}

After reaching the blood-current the virus has the property of passing through the walls of the blood-ressels by osmosis, and is deposited at varions points in the tissues. Wherever the virus is deposited local inflammatory infiltration occurs.

Is the rirus is in these various spots, their secretions and detrilus can produce syphilis if inoculated into another individual. As a conseyunce of the growth and activity of the bacteria which we assume to be the canse of syphilis, and which are believed to increase and multiply in the chancre, lymphatic glands, and all secondary lesions, toxins are produced which have a poisonous effect upon the nervous system, and occasion ferer, headache, and backache.

\section{STAGES OF SYPHILIS.}

It is found convenient to divide the regular course of syphilis into three stages. as follows:-

I. Primary stage, which includes the time from the moment of infection to the outbreak of general symptoms (eruption and mucous patches. otc.), and which last from eight to ten weeks.

For the first three weelis of the primary stage there are no symptoms to indicate that the individual contains the virus of syphitis in his tisucs. At the end of three weeks the chancre appears, and the adjaent glands become enlarged and are the only signs of infection.

II. The secondary stage begins when the eruption, alopccia, and mucous patches make their appearance. It is the stage of typical and regular derelopment of exuptions and their accompanying symptoms, and prinds of latency alternate with relapses. The secondary stage last-from six to eighteen montlis, or abnut one year on the average.

III. The tertiary stage comes nn after a prolonged period of 
latency, and is the stage of formation of gumma. The trutiary stage may never occur. Its seeretions are not contagions, and it resulules a diathesis more than an active disease.

\section{INCUBATION.}

Definition.-The period of time which elapses after the poison has entered the body and until the first manifestation of its working appears. The poison is not inactive during this time, but increasing in quantity.

The primary stage of syphilis is divided into two periods of incubation:-

(a) Period of primary incubation, which is the period from the time of infection until the chancre appears. The chancre represents the reaction of the tissues in consequence of the local increase of the virus.

(b) Period of Secondary Incubation.-This is the time from the appearance of the chancre until the eruption is seen. The eruption indicates that the blood is saturated with the virus.

\section{GLANDULAR ENLARGEMENT.}

The lymphatic glands are supposed to act as places for the incubation, growth, and development of the syphilitic virus and to serve as store-houses for it, during the entire secondary stage. The glands nearest the chancre begin to enlarge about four weeks after infection.

General Characteristics.-The glands become:-

(a) Firm and hard.

(b) Freely movable under the skin.

(c) No pain.

(d) Rarely suppurate except in strmons or weak patients.

The glands nearest the chancre become enlarged first; consequently we find that, when the chancre is located on the penis, the inguinal glands enlarge first; when the seat of the chancre is on the breast, the axillary glands are the first to become alfocted; and. if the chancre is located on the tongue or lips, the sutumurillary glands are first attacked.

The lymphatic vessels lying between the wands aften hecomm. hard and indurated, feeling like cords. and this comdition is called lymphangitis. 
Course of Virus Through the Lymphatic System.-The syphilitic virus pursues the following course, in its progress through the lymwhatic ressels into the general blood-circulation:-

The small round-celled infiltration, which occurs in the chancre, closes up the mouth of the blood-ressels and temporarily holds in check the spread of the poison. The multiple swelling of the lymphatic glands also retards the entrance of the virus into the bloodcurrent.

After the virus begins its increase in the chancre, a part of it is carrich to the nearest lymphatic gland, which is a favorable spot for its growth and increase. Part of the virus which is deposited here remains and a part travels farther to the next lymphatic gland.

When the chancre is located on the penis, the course of the virus is as follows: Through the inguinal glands, lymphatic glands in the abdominal cavity, receptaculum chyli, and thoracic duct, from which it is poured into the left subclavian vein and general blood-circulation.

During this time the cervical and axillary glands have had none of the virus passing through them. After the blood has become charged with the virus, they filter out the poison, are infected, and become enlarged in consequence.

\section{SECONDARY SYPHILIS.}

PRODROMAL SYMPTOMS, OR PRODROMATA.

The virus of syphlilis has the power of destroying the red corpuscles after it has entered the blood-circulation, and on microscopic examination we find that the red corpuscles are diminished in number, with a decrease of hamoglobin, and that the number of leucocytes is incrased. In concepuence of these changes the stin and mucous membranes appear pale and anamic.

Fever is present in nearly erery case. It is usually slight in wellnomrished ank strong individuals, but in debilitated subjects may reach $103^{\circ}$ or $104^{\circ} \mathrm{F}$.

Nocturmal Pains. - The shafts of the long bones, such as the tilia and rilse and the vertex of the cranium are usually affected with pains whing are more or less severe, and which have the peculiarity of rrmilling through the da!l and coming on at night, and reach their wn rimum intensit!l atout minnight. 



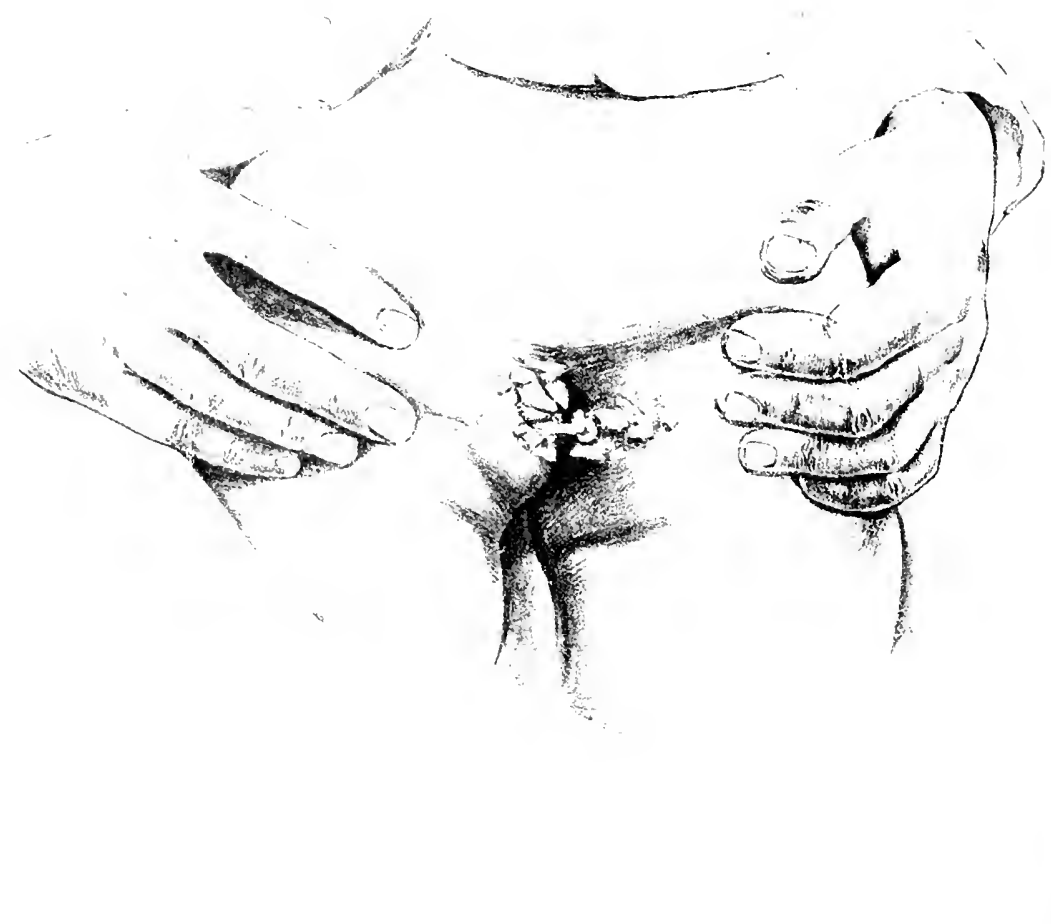


The pains are boring or tearing in charater, and are callen osteocopic pains (bone-tearing).

Jaundice occurs in a small proportion of cises, anul, whiln its cause is not definitely ascertaincd, it is assumed to be due to cilluch (a) pressure upon the common bile-duct, from the enlargerl lymplaitic glands lying in the abdomen, or (b) from congestion and swelling of the mucous membrane lining the common bile-duct, as a result of the disturbed circulation.

Albuminuria ocenrs occasionally. It is temporary in character and is due to congestion of the kidneys.

Erythema of the fauces is a most constant symptom, and appears as a generally diffused erythematous redness upon the pillars of the fauces and the pharynx.

All the symptoms described above under the term prodromata appear before the eruption and mucous patches, and are all occasioned by a disturbance in the local blood-supply, which induces a condition of hyperæmia or active congestion in the rarions organs affected.

The mucous patch is one of the most constant and characteristic lesions of secondary syphilis. It makes its appearance about the same time that the eruption is observed. In the earliest stage the moncons patch appears as a pearly-white round spot upon the nucous membrane of the mouth, entrance to the ragina, margin of the anms, or under the female breast. Its development may occur wherever the skin is thin and delicate and kept macerated by secretions.

As it is seen first, the mucous patch looks as thongh nitrate of silver had been brushed orer the surface. After a litule time. the infiltrated pellicle of mucous membrane sloughs ott. learing a shallow, sharply-defined, "punched-out-looking" nlcer. 'T'his might be rogarded as the second stage of the process, and is the form in whints the mucous patch is most commonly secn.

The condyloma may be looker upon as the third stange in the development of the mucous patch, and is bronght ahout he the active growth and proliferation upon the floor of the mlecr. The result is the formation of canliflower-like granulations. Which rise in lith hillocks. above the level of the surounding healthy skin.

The secretion which is very alumetanty fumishod by mucoms 
latches and condylomata is highly contagious, and is responsible for the transmision of most of the eases of syphilis.

\section{ALOPECIA.}

In many cases of early secondary syphilis, although not invariably, the hair falls out to a greater or less degree.

It is due to two different causes:-

(a) The impaired quality of the blood fails to afford sufficient nourishment for the hair-bulbs, and they die.

(b) An increased secretion of sebum takes place around the bulb, and by its pressure shuts off the circulation of the nutrient fluid in the shaft of the hair.

Alopecia occurs in various forms. It may be complete, in which case the hair of the head, eyebrows, and pubes may fall out entirely. 'I'] le usual form, however, consists in a patchy baldness, the hair falling out in small patches of the size of a finger-nail. In some cases there is only a general thinning of the hair, without the formation of any distinet bald patehes.

The prognosis is good, and the hair always grows in again, unless an ulcerative process has taken place upon the scalp and destroyed the hair-follicles.

\section{SKIN ERUPTIONS, OR SYPHILIDES.}

The appearance of the rash upon the skin announces that the bloor is saturated with the virus of syphilis. The eruption is suppoeed to he cansed primarily by the irritating effect of the virus upon the rasomotor centres, causing a local paralysis and temporary dilatation of the arterioles in the skin, with inflammatory changes within and around the ressel, at the spot where each lesion is located.

Microscopically all the eruptions of syphilis are identical, and rniy in ontwarl appearances only becanse of the difference in the ament of cellular infiltration.

On microscopic examination all the lesions are found to be due to tuo distinct morbid processes:- 
(a) Inflammation in the onter and imner coats of the arterules, accompanied by stasis of blood or hyperæmia in the aprillaries.

(b) An infiltration of small round cells, as the result of the inflammatory process, which begins in the arterioles and sureals outward toward the periphery, in the tissues surrounding the resclts. It times the changes of arteritis and cellular infiltration are so speat that the circulation of blood through the ressels is cut olf, and the parts supplied become the seat of coagulation-necrosis and slounh out.

Classification and Anatomy of Eruptions 0ccurring in the Secondary Period. - I. Macular Syphitide. Synonyms: Syphilitic erythema; syphilitic roseola.

The pathological change in the skin which is the seat of a macular syphilide consists in periarteritis and endarteritis, with an exeess of blood in the capillaries; but, while the romnd-celled infiltration has taken place in the tissnes, it is so slight in amount that it can only be detected by microscopic examination.

When a considerable amount of round-celled infiltration has bcen produced, it is readily perceptible, and forms nodules or small tumor's, which are spoken of as the

II. Papular Syphilide. - The papules forming this variety of syphilide may be as large as a 10-cent piece or as small as a pin's head, and are accordingly classified as the large or smull pumutur syphilide.

In certain cases the epidermis covering the papule scales off, but remains partly attached, and the syphilide is then spoken of as papulosquamous.

If the centre of the papule becomes necrotic and breaks down, a funnel-shaped depression is formed, which is filled with pus and corered over by a scale of epidermal corering. This variety is known as the papulo-pustular syphitide.

III. The Pustular Syphilide.--The pustnlar syphilide is formet from a pre-existing papule whose central part undercoes necrosis and, as a result of obliteration of the arterioles from endarteritis and periarteritis, sloughs and breaks down. The process is then complicaterl by the inoculation of progenic micro-organisms. Thus canse a suppuration of the central part of the papule, and the alcimulation of pus, which forms underneath a covering of epizermis. which is raised up above it, preventing its escape.

IV. The Pigmentary Syphitite.-This form of sylhilide should 
not be confounded with the pigmentation which renuins after the disappearance by absorption of a papule.

The pigmentary syphilide occurs as an independent eruption upon a spot which was not previonsly affected. It is oceasioned by the mularteritis and periarteritis, which allows the red corpuscles of the blood to cscape through the ressel-walls into the tissues. 'The hemochlobin of the escaped blood becomes dark brown in color, oceasioning a pigmented appearance of the skin, and is ultimately absorbed, leaving the skin whiter in appearance than before.

The pigmentary syphilide occurs in tuo forms:-

(a) As dark spots or patches of brown pigmented skin.

(b) As a diffuse, wide-spread, dark pigmentation, with areas of healthy white skin seattered here and there through it. In this form (b) absorption of the hrmoglobin has taken place in eertain areas of the pigmented patch, leaving white spots free from pigment.

Distribution of Syphilides over the Body.-The portion of the skin attacked and the extent of the eruption depend upon the age of the syphilis.

The early eruptions which occur during the first six months are:-

(a) Superficially seated in the skin.

(b) Generally distributed over the body, appearing first upon the chest and abdomen and spreading subsequently to the palms and soles.

(c) The eruption is symmetrical, occurring alike on both sides of the body, and tends to arrange itself along the course of the connective-tissue bundles which lie under the skin (called lines of cleavage).

(d) The early eruptions genarally disappear spontaneously by absorption.

Relapsing Syphilitic Eruptions. - The relapses occur after the first six months, and differ in the following particulars from the early eruptions:-

Distribution.-(a) While they may be distributed over the entire lindy, the relapsing eruptions are never so numerous as the early lesions.

(b) They do not follow the lines of cleavage, but are arranged in circles or segments of circles.

(c) The groups of lesions do not tend to arrange themselves symmetrically, as a rule. but have certain spots of predilection, such as the gunital: and ants, the mucous membrane of the mouth, the palms and 


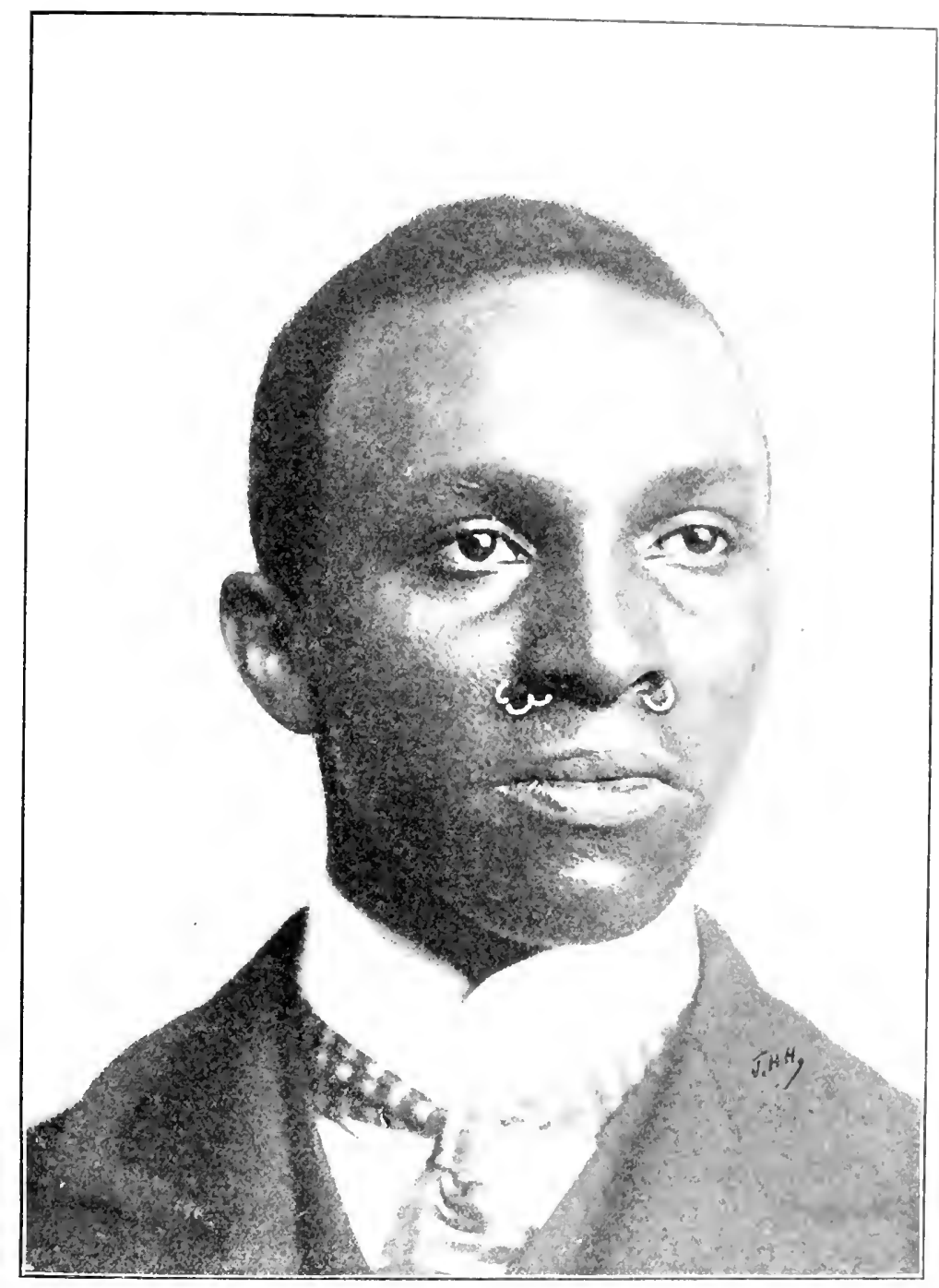

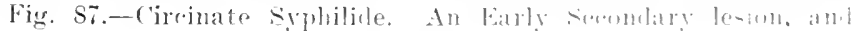

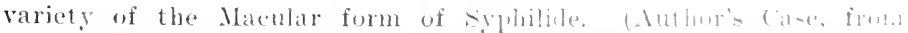
polhemus (linic) 




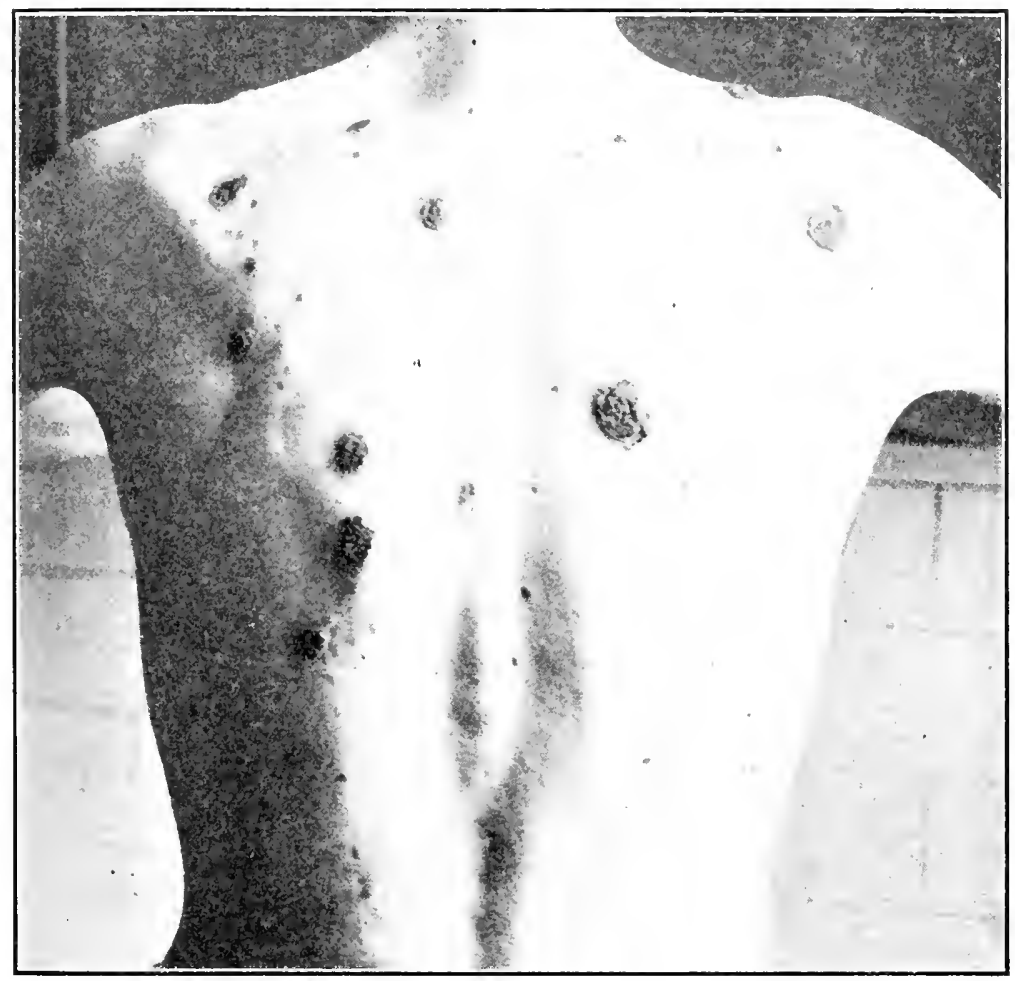

Fig. 89.-Malignant Syphilis. Pustular Eruption. (Author's Case, from Polhemus Clinic.) 

soles, the upper margin of the forchead near the liair, and the flexures of joints.

(d) The later eruptions are less apt to be absorbed than the carly lesions, but tend rather to break down and ulcerate.

Course of the Eruption. - Syphilides are temporary formations which grow and spread at the periphery, or outer eslge, and after reaching a certain height absorption or ulceration takes plince, beginning in the centre.

In this way the ring formation occurs. The centre, or olitest part, has been absorbed and disappeared, leaving the most recently formed portion of eruption still present as a ring around the healed centre.

Retrogressive Changes of Syphilides.-As stated above, the process of absorption always begins in the centre of the lesion.

'The macular syphilide disappears most readily, and, if a pigmentation were formed, it is soon absorbed, leaving the skin whiter than normal.

In the papule the central part is absorbed, leaving a depression, which is filled with epidermal scales.

T'he pustule is formed from a papule, and has a crater-like depression in its centre filled with pus; this dries up and forms a crust, and the surrounding ring of infiltration is removed by absorption.

The papule and pustule leare traces of their presence, as spots of pigmentation, which remain on the site of the original lesion for a long time.

If the pustule has caused much necrosis of the normal cutaneous elements, a cicatrix will be formed.

Symptoms of Syphilides. - I. Arrangement of the lecions. II. Polymorphism. III. Color. IV. Absence of burning and itching.

I. The arrangement has already been considered, and kepends entirely on whether the syphilide is early or one of the latur rolapsing eruptions.

II. Polymorphism is the most valuable diagnostic sign of a sypliilide, and pertains to both early and late rarietics of ermption. Polymorphism may be defined as direrse as to form, and is lurought ahout in the following way: The ermption in syphilis nerer comes out all at once, but makes its appearance in successive crops. Then the wuption is viewed, if it has lasted for some little time. it maty he noticent that the older spots have undergone transformation. while in the later spots the changes are less marked. Hence we find present at the same 
time macules, papules, and pustules, and the lesions are all of different ages and various stages of development or retrogression.

III. Color.-The color of syphilides is frequently compared to that of an old copper cent or of lean raw ham. The color is by no means pathognomonic, as it occurs in most inflammations of the skin which are chronic. It is occasioned by the arteritis, which weakens the vessel-walls and allows the red corpuscles to pass out into the surrounding tissues. The hæmoglobin loses its bright-red color and becomes brown.

$I \mathrm{~V}$. The absence of burning and itching is not invariable, but is usual, and is accounted for by the slow chronic character of the derelopment of the lesions.

Duration of Secondary Stage.-The secondary stage lasts a variable length of time, which may be from six to eighteen months, and may be set down as about one year, on the average.

In cases which follow a favorable course relapses cease to occur, and the disease appears to be at an end.

It is not possible to draw any distinct line of demarcation between the sccondary and tertiary periods, for as the lesions continue to recur they gradually lose the distinguishing characteristics of secondary syphilis and become distinctly tertiary.

In the secondary stage the visible manifestations of the disease occur chiefly upon the skin and mucous membranes, and, while the riscera, eyes, and nerrous system are occasionally attacked, they are not apt to be affected.

In the tertiary stage, however, the affections of the skin are trivial in comparison with the damage which may be wrought in other vital organs.

\section{TERTIARY STAGE.}

Individuals who are strong and well nourished and who are systematically and carefully treated for a sufficient length of time rarely develop tertiary symptoms.

Tertiary lesions are said to occur in from 5 to 40 per cent. of rases of syphilis. The most nsual time for their appearance is from three to five years after infection, although long periods of latency, 


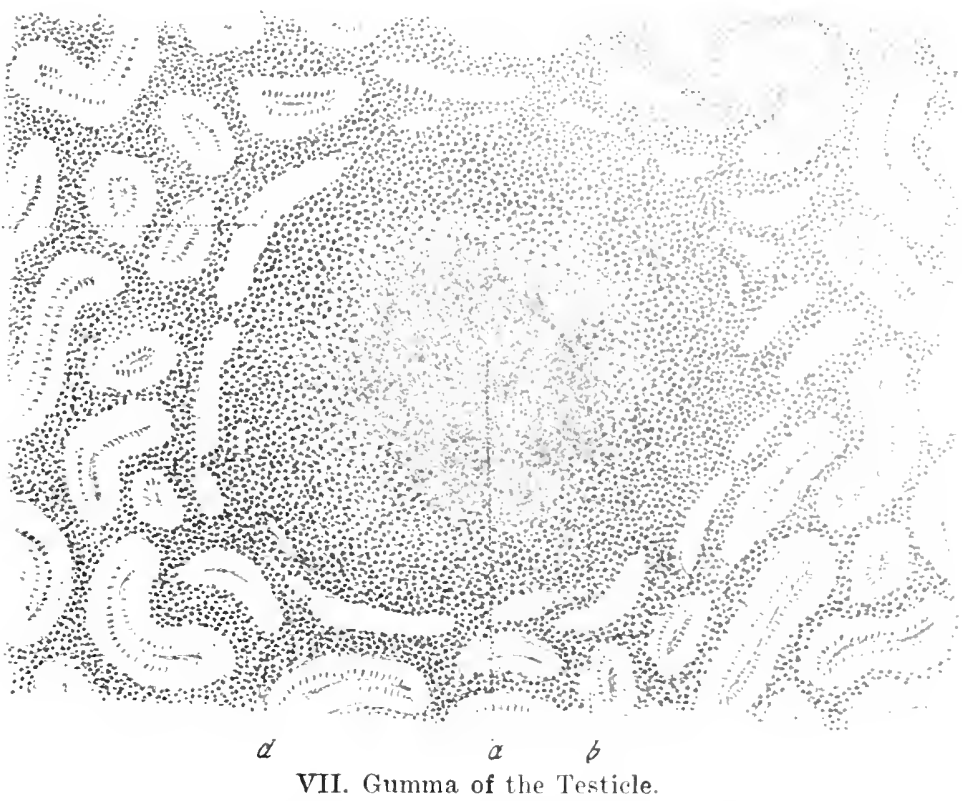

a, Central porton, seat of eoagulation-necrosis. b, leripheral zone formed hy infiltration of small ronnd sells $c$. Cimpressed tubuli seminiler. $\quad d$, Interstitial small round-celled infiltration

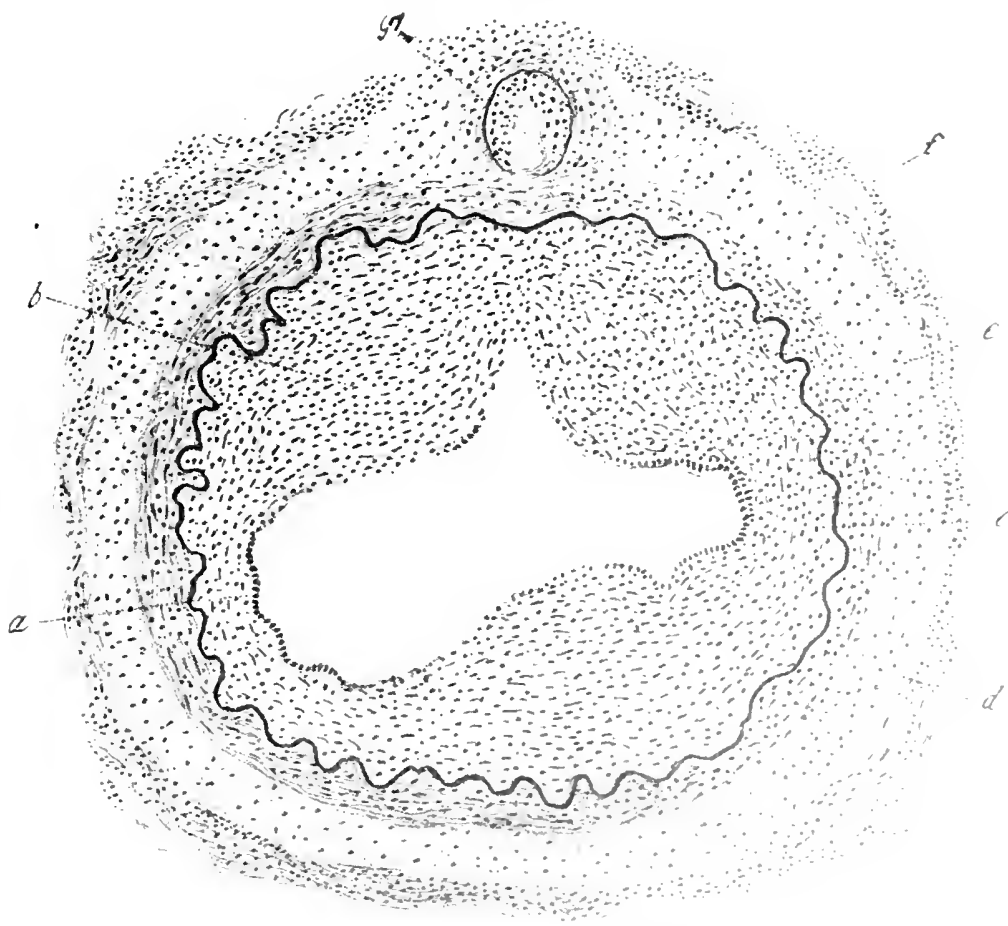

VIII. Endarteritis (Artery from the Fisule of cillinat.

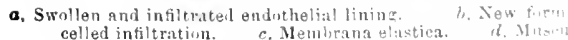

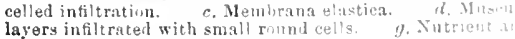



even as mueh as fifty years, during which the patient is entirely free from symptoms, may intervene before the tertiary lesions appear.

Characteristics of Lesions of Skin and Mucous Membrane.-The characteristics of the syphilides in the tertiary period ditler greatly from the secondaries, and may be summarized as follows:-

I. 'They attack a limited area.

II. They have a tendency to extend and eause destruction of tissue, with the formation of sear-tissue and its subsequent contraction.

III. 'They do not tend to spontaneous recoresy, but rather breali down and ulcerate.

IV. They extend deeply into the tissues.

Tertiary lesions, in whatever organ or structure of the loody they may occur, are the direct result of chronic vascular lesions, and are commonly accompanied by the so-called gumma.

\section{PATHOLOGY.}

Arterial Changes. - In the tertiary period of syphilis arteritis plays an important rôle. In the secondary period the inflammatory changes in the vessels are more apt to involve the adrentilia, but in tertiary syphilis the inner coat is more liable to be attacked.

The chronic inflammation of the ressel-walls cances an infiltration of small round cells to take place between the inlima and the endothelial lining of the artery, and this infiltration ultimately becomes organized into connective tissue. The contraction of the tissue thus formed causes a narrowing of the calibre of the artery, and, indece. it may lead to the total closure of its lumen, and the ressel becomes transformed into a solid string of comnective tissue (endarteritis obliterans).

When the arterial wall is the seat of an inflammatory process which is very circumscribed, instead of its lmmen lecoming narromed or obliterated its walls may be stretched and dilated, and in this way small miliary aneurisms are formed.

Gummous arteritis is a rare aflection, consisting in the formition of small gummata, which grow from the media and push rupird. covered by the unchanged intima, into the lunen of the artery. "The centre of the gumma generally hecomes chees amb hrualis dom. Obliterating endarteritis can also exist at the sanue point in the resere in addition to the gumma. 
Gumma ${ }^{1}$ is always preceded by and dependent upon the arteritis. It occurs especially in the slin, periosteum, meninges of the brain and cord, and the abdominal viscera, particularly the liver, spleen, and testicle.

The gumma consists of a circumscribed mass of new tissue, varying in size from a pin's head to a hen's egg, and is composed of a hyaline matrix, in which are imbedded small round cells and occasionally giant cells. Small gummata may disappear by absorption, but, in gummata of larger size, the nutrition of the central parts of the tumor is cut off, by the pressure of the liyperplastic tissue on the blood-ressels and by the accompanying endarteritis, and the centre of the tumor undergoes coagulation-necrosis.

After a gumma has lasted for some time, it is found to be composed of an old central part made up of fatty, cheesy, broken-down cells and an onter zone which has been transformed into a fibrous connective-tissue envelope surrounding the softened sloughing centre.

A gumma may exist singly or there may be several present, lying clnse together. The cireumscribed form of gumma developing in the skin is termed a syphitilic tubercle.

\section{CLASSIFICATION OF SKIN ERUPTIONS.}

The eruptions which oceur upon the skin in tertiary syphilis are all occasioned by the formation of gummata in the skin or subcutaneous connective tissue and their subsequent progress of ulceration, and are classified as follows:-

I. Gumma: (a) Of the skin. (b) Subcutaneous tissue.

II. Tubercular syphilide: (a) Dry or atrophic. (b) Ulcerative.

III. Rupial syphilide.

(a) Gumma of the Skin. (b) Gumma of the Subcutaneous Tissues.-Gumma of the subcutaneons tissues is the canse of the deep

'The name of infectious granulomata was given by Virchow to a class of diseases whose derelopment does not pass beyond the stage of formation of gramulation-tissue, which is transitory in the character of its duration and cnds by ulceration. In addition, it is nearly allied in its formation to the proces of inflammation. The class of infections granulomata includes syphilic, tubereulosis, leprosy, actinomycosis, mycosis fungoides, rhinoscleroma, and granders. 

rilcerations commonly met with in tertiary syphilis. The skin lying above the gumma becomes attached to it by the inflummation, sofums: and sloughs away, exposing to the air the cavity which was formerl in the central part of the gumma, by the coagulation-usecrosis of the new infiltrating cells. As the ulcers heal, a cicatrix is formen which dips down into the cavity of the gumma and a depressed scalr. attached firmly to the subcutaneous tissues, remains at that point.

The circumscribed form of gumma which develops in the skin is termed a syphilitic tubercle. This condition is entirely distinct from tuberculosis of the skin, which depends upon the tubercle bacillus, and in this instance the word tubercle means a little tulyer, or small nodule. The tubercular syphilide appears in two forms:-

(a) Dry, or atrophic, tubercular syphilide, which is so called from the fact that it does not break down and undergo destruction, but tends to dry up and disappear by absorption.

It is this variety of syphilide which occasions the ringed form of eruption which comes on late in the course of the disease. (See plate.)

(b) The ulcerative form of tubercular syphilide takes its name from the fact that, unlike the atrophic form, it is not absorbed, but breaks down and ulcerates, continually spreading farther at the edge and healing in the contre. From its tendency to extend at the periphery and involve fresh areas of tissue it is sometimes termed the serpiginous syphilide.

The rupial syphilide derives its name from the concentrie lings of crust which form the scab, resembling the rings of an oyster-shelf. The formation of the rings occurs in this manner: A smill gumma forms in the skin, ulcerates, and the ulcer is covered with a crust. The ulceration extends at the periphery; a ring of crust is formed over the new ulceration and lies underneath the first crust, and as it, is larger in size, projects all around it. As the ulceration extends, other layers of crust are formed molemeath the original ones. and each additional crust which forms is larger in diameter than the one preceding it, and is not entirely covered, so that the edge is seen forming a ring around the crust above it.

The lesions above described are all deeply seater, involve the subcutaneons tissnes, and result in more or less destruction of tissule, which is healed by the formation of onntrating cicatrices. 


\section{SYPHILIS AND IRRITATION.}

The predilection which syphilitic lesions seem to show for cer. tain structures may be partly accounted for in the following way: It has long been noticed that any irritation or trifling form of injury in a syphilitic individual predisposes toward the formation of lesions at the injurcd or irritated point. Frequent examples may be called to mincl, as, for instance, the effects of smoking or the friction from broken tecth in causing sores in the mouth. Pressure contact, if oft repeated and prolonged,-as, for example, the pressure of the forearm upon a dcsk, in a clerk or book-keeper,-predisposes to the appearance of an eruption upon the skin of the forearms. A slight blow or squece may induce the formation of gumma in the bones or testicles, and, as would natmally be expected, the bones which lie near the surface of the slin-such as the tibia, ribs, and sliull-are more apt to be aftected by periostitis than the bones which are abundantly covered by a thick cushion of muscle and fat.

Syphilitic lesions also, by preference, affect the point of least resistance in the organism. Thus, for instance, syphilis of the liver is more frequent in alcoholies than in abstemious persons, and syphilis is particularly liable to attack the brain and its meninges in individuals who are mentally active and are brain-workers. For the same reason a relapse of a syphilis, which has been latent for some time, is often induced by an attack of malnutrition brought on by want and privation.

\section{MALIGNANT SYPHILIS.}

Syphilis is said to be malignant when it pursues a rapid, destructive, and uncontrollable course from the outset, and it only occurs in individuals who are debilitated from tuberculosis, alcoholism, or bad nutrition and privation. Its severity is increased from the fact that such cases do not tolerate the administration of mercury and iodide of potash well.

The early lesions are generally distributed over the body, are pustular in character, break down, and cause extensive ulcerations. The animia from the destruction of the red corpuseles is very marked, 


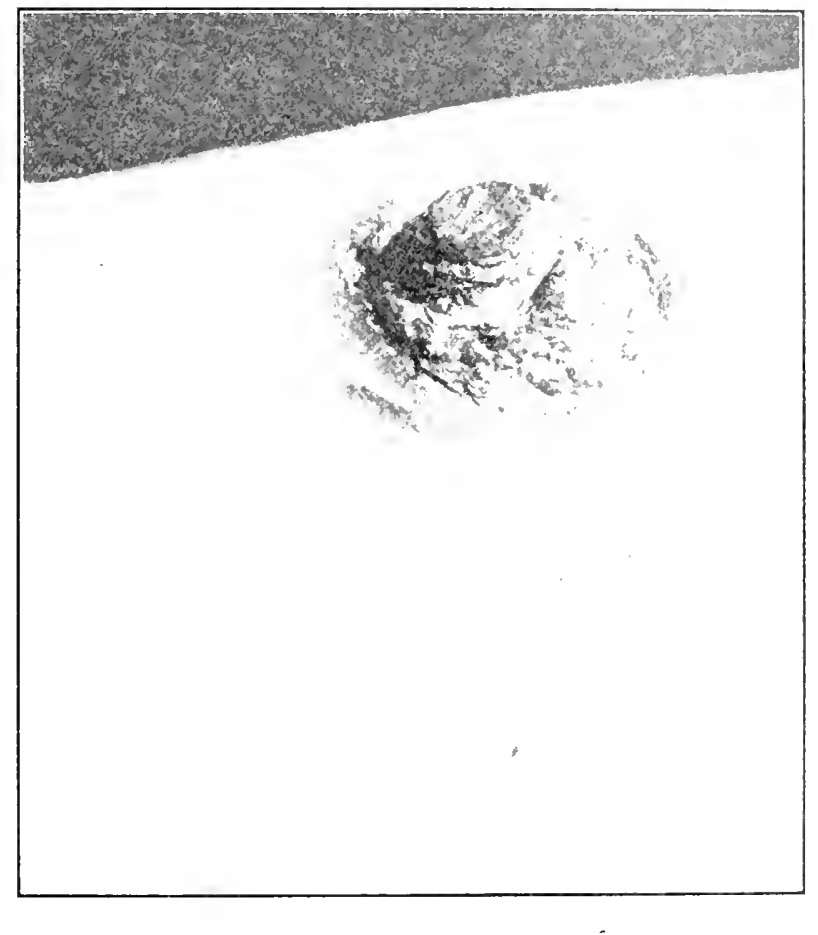

Fig. 92.-Rupial Syphilide. (Author's Case, from Polhemus Clinic.) 


and the cachexia is strikingly evident. The subcutaneous fat is absorbed, and the patient emaciates.

Gummata develop prematurely, and four or five munthis after infection the most extensive, wide-spread forms of gummatons ulcrintion may be present. Death may result from destruction of sime vital organ, or the prolonged suppuration of the ulcers may cause death from amyloid or fatty degeneration of the viscera or ceneral marasmus.

If the resistance of the system be enough to withstand the drain for two years, the virulence of the disease is exhausted, and the patient may live, but in a weak and debilitated condition of body.

In the foregoing sections we have considered the manifestations of syphilis in the skin and mueous membranes only, but, as has already been indicated, the lesions of syphilis are not confined to any one structure.

The pathological processes of endarteritis and periarteritis, with their accompanying cellular infiltration of tissue and the resulting degenerative changes in neighboring structures, as well as the gummatous infiltration occurring in the later period of syphilis, are liable to attack any organ in the body, and the symptoms resulting repend wholly upon the function of the organ attacked.

For syphilitic affections of the muscles, joints, and breast the reader is referred to the treatises on surgery, and to the works on internal medicine for an account of syphilitic disease of the liver, heart, spleen, etc.

The diseases of the nerrous system, eye, and ear which depend upon syphilis for their causation are best studied in the special textbooks deroted to those subjects.

\section{IMMUNITY IN SYPHILIS.}

Immunity may be definer as the condition of bod!n which rexists the grouth and pathogenesis of disease-producing germs.

As in all other infectious diseases. one attack of syphilis protects against others, and it is an event of the greatest rarity for a second 
infection to occur. The essential nature and cause of immunity, in any infectious discase, is still a matter of speculation. There are three theorics at present to account for it:-

\section{Phagocytosis.}

II. The bactericidal power of the blood.

\section{The antitoxins.}

The latter theory is not fully established, but is more generally accepted than either of the others. It is known that bacterial action and growth produce substances called toxins, which are poisonous. It is supposed that another set of chemical substances called antitoxins, or defensive proteids, is being formed at the same time. The antitoxins do not destroy the toxins, but exert some action upon the tissues of the body which causes them to be insusceptible to the action of the toxins.

Immunity may be acquired in three ways:-

I. By means of a first attack.

II. By means of heredity, which is the so-called inherited immunity, and is demonstrated by the fact that occasionally healthy children are born of syphilitic parents, because the fœtus has acquired its immunity in utero.

III. Acquired Immunity.-It sometimes happens that healthy mothers who become pregnant with a syphilitic child (infected with syphilis and generated by a syphilitic father at the same time) may acquire immunity through alsorption of the syphilis antitoxins which had been formed in the foetus.

\section{INHERITED SYPHILIS.}

Infection.-Father and Mother both Syphititic.-It is almost needless to say that, when both father and mother are syphilitic at the time of conception, the fotus will be almost invariably infected. The children suffer from a serere form of syphilis, and usually die.

Wother alone Syphititic (Father being Healthy). - If the mother acunired her syphilis before conception the child is almost sure to be infected, as it is nourished directly by the maternal blood containing 
the syphilitic virus. If the mother is infecter with syphilis after conception, but during the pregnancy, we have two conclitions to consider:-

(a) If the mother's infection has occurred in the carly months of pregnancy, before the independent fotal circulation has been established, the fotus is usually infected with syphilis, because the fotus is nourished directly by the maternal blood containing the syphilitic virus.

(b) In the later months of pregnancy, however, the foetus has its own independent circulation. The villi of the placenta are interposed between the fotal and maternal circulation, and the nutrient material passes from the mother to the fotus, not by direct interchange of blood, but by osmosis.

If the mother acquires syphilis after the establishment of an independent fœtal circulation, the villi of the placenta may filter out and prevent the virus from reaching the child; but, as the antitoxins are held in solution, they will pass through the membrane of the placental villi and exert an immunizing action upon the fœtus. Hence we have Profeta's law of immunity: "Children may be born of syphilitic parents and remain healthy, and present an immunity against syphilis which is either absolute or else modifies the syphilis so that it runs a very mild course."

Father alone Syphititic (Mother being Healthy).-Under this head there are also two conditions to consider:-

(a) The spermatozoa may contain the virus and carry it to the ovum at the time of conception, and, as a result, the child is syphilitic. The syphilis may cause the death of the foctus in utero, interference with development, or simple debility, or the child may be born syphilitic.

Infection of the fotus by the father is the nost common form. but the syphilis resulting is less severe than in the other forms of infection, for the reason that if the mother remains healthy the fuetus is nourished with healthy blood and the harmful effects of the syphilis are partly neutralized.

(b) The spermatozoa, however, do not ahrays carry the virus of syphilis to the ovule, and the child often escapes infection and is borm healthy.

Influence of the Child's Syphilis as Exerted upon the Mother.We may consider now the instances in which the mother is fres from syphilis, but the child has been infected by the fulher at the timo of 
conception. The effects which the child's syphilis cause in the mother must be divided into three groups:-

(a) In the first group, the mother remains entirely unaffected, and a condition of immunity is not established, so that the mother may acquire syphilis from her orm child after its birth or from other sources. These cases are extremely rare, and prove an exception to the generally established law of Colles.

(b) The second group forms a large number of cases, and comprises the instances in which the mother is infected with syphilis from her own child, during its term of gestation in the uterus. The name given to this mode of infection of the mother is choc en retour, or syphitis by conception. 'The syphilitic virus formed in the child passes orer directly into the mother's blood, through the placental circulation.

(c) The third group includes the cases in which the mother is not infected from the child in utero, but becomes immune against s!phitis, and is described by Colles's law, riz.: "A syphilitic ehild cannot infect its own mother after its birth." The mother acquires immunity against infection, probably from absorption of the antitoxin of syphilis which is formed in the body of the foetus. It is supposed that the placental villi act as a filter, and allow the antitoxins, which are in a state of solution, to pass through them, by osmosis, into the maternal blood, but prevent the virus from reaching the mother's circulation in any great quantity.

Length of Time After the Chancre at which Infection of the Fœtus or Choc en Retour is Liable to 0ccur.-Syphilis is most liable to be communicated to the fœtus or by choc en retour during the secondary period and the first three or four years after the primary sore. As time passes the intensity of the syphilis also diminishes, as is shown by the following table:-

Termination of Pregnancies in a Syphilitic Mother. - I. First pregnancy terminates in an abortion.

II. The next pregnancy results in the premature birth of a syphilitic child.

III. Then follows the birth of a full-term child, which has syphilitic manifestations.

IV. Next a child is born at full term, which subsequently develope manifestations of syphilis.

T. Finally healthy, full-term children are born which remain licalthy and free from syphilis. 
It is important to bear in mind that the inheritance of syphilis by the child can be farorably influenced or absolutely prevented by treating the syphilis of the parents, both before and after conception, with mercury.

As a result of the observations made in regard to inherited syphilis, the following principles can be applied practically:-

I. A man or woman with syphilis should not be allowed to marry until three or four years have elapsed since the original infection, and methodic treatment should be carried out during this period.

II. If a married man or woman acquire syphilis, he or she should be energetically treated, in order to lesson the danger of infection of the fotus in case pregnancy should occur.

III. A pregnant woman with syphilis should be energetically treated during the entire period of pregnancy.

IV. If a woman free from syphilis becomes pregnant, by a syphilitic man, she should be treated, to prevent the danger of choc en retour.

V. A syphilitic child should only nurse from its mother, and never be allowed to nurse from a wet-nurse.

VI. A child apparently healthy, although born of syphilitic parents, should never be allowed to nurse from a wet-nurse, until at least three months have elapsed without any symptoms of syphilis developing in the child.

\section{SYPHILIS AND MARRIAGE.}

The relation which the question of marriage bears to syphilis is an important one. The lesions of tertiary syphilis are not contagious, and in most eases the infectious element has disappeared after the lapse of two years; still it is necessary to bear in mind that a syphilitic father may propagate a diseased child, which may be still-born or infect the mother by choc en retour, up to the end of the third or fourth year. On this account a set of morking rules might he formulated as follows:-

I. No one showing signs of active syphilis shonld he allowed to marry, even though more than four years have elinpsed since the primary infection. 
II. Narriage should never be sanctioned until at least three years have elapsed after infection, provided the patient has been systematically treated during that time: but four years is a safer time-limit, and patients should be advised to wait for that length of time.

III. Marriage should not be permitted until at least one year has passed during which no symptoms have appeared.

\section{DIAGNOSIS OF SYPHILIS.}

The question of diagnosis is an important one, both from the stand-point of therapeutics and also from the necessity of guarding other persons against a contagious disease. Of course, it is more difficult oftentimes to make a diagnosis many years after infection than when the initial lesion, mueous patches, and cruption are all present. It is always better to conduct the examination in a systematic manner, and begin by taking the history of the case.

We should inquire if the patient has suffered from: $(a)$ a venercal sore with lumps in the groin; followed by $(b)$ a rash upon the skin, $(c)$ sore throat or sores in the mouth, $(d)$ pains in the bones and skull which were worse at night, $(e)$ sore eyes, and $(f)$ in women the oceurrence of abortion or still-births.

It is important to bear in mind that syphilis is not always aequired by renereal contact, and the initial lesion may not have been on the genital organs. We should also remember that the early manifestations may have becn so slight, particularly in women, as not to have been noticed.

It is well to bear in mind that in men there is a possibility of the chancre being located within the urethra, without causing any symptoms except a slight gleety discharge, which might be mistaken for a urethritis. The glandular cnlargement, which is such a valuable dianostic sign, disappears after two years and is unarailable.

Late in the tertiary period the diagnosis often presents great difficultics. 'There are, howerer, certain points for examination which may throw some light on the nature of the case:-

The stin and mucous membranes should be examined for cicatrices. The bones and testes may show irregularities or swellings. The cyes often slow decided changes. Local paralysis of an ocular muscle is a valuable diagnostic sign. Iritic adhesions may be present, or there 
may be changes in the deeper structures. B. Sachs regards the artion of the pupits as a very important sign. The changes which oceur in syphilitic cases, without previous demonstrable ocular dicease, are as follow: I. Inequality of pupils. II. Unequal response to light in one pupil, but not in the other. III. Complete immobility to light and accommodation. IV. Departure from the circular form of the prupil without preceding iritis.

In cloubtful cases the diagnosis is sometimes marle by the cifects of the administration of mercury and iodide of potash. If the lesions improve, it is supposed that they were of syphilitic origin. This is a very uncertain and misleading method of trying to get at the truth, for the reason that mercury and iodide of potash will often canse the absorption of newly-formed inflammatory infiltration, irrespective of its cause. The results of an inflammation which was due to the irritation of any toxic agent in the blood-either uric acid, alcohol, or syphilis-will be absorbed under the use of mereury and iorlide. although the infiltration due to syphilis disappears more quickly than the others. (See chapter on "Inherited Syphilis.")

\section{PROGNOSIS OF SYPHILIS.}

The danger to life in syphilis in the adult depends upon the involvement of some vital organ by endarteritis or gummatous infiltration, and this frequently occurs years after infection, when the syphilis is supposed to be extinct.

The ultimate recovery of a patient with syphilis repends upon the following factors:-

I. On the systematic thoroughness and length of time which the case is treated.

II. On the constitution of the patient.

III. On the virulence of the poison.

Any cause which tends to depress the general health-such as privation, bad hygienic surromndings, orerwork, anxiety, less of sleep, and particularly habits of alcoholic indulgenee-retart recovery.

As in most other diseases, the extremes of life-that is, old persons and young children-hear syphilis badly, and the death-rate, particularly in children, is high.

Women are believed by some German obeervers to be lus seresely 
affected than men, and are thought to be less liable to syphilitic affections of the nervous system.

Among the early prognostic indications it is thought that, when the glandular enlargement is slight, the syphilis will run a mild course.

The appearance of tertiary lesions prematurely, and in the early months after infection, is a rery unfavorable prognostic sign.

Extragenital chancres-i.e., those which are located on the finger, breast. lip, etc.--are more apt to be followed by a severe attack of syphilis than in the cases where the chancre is located upon the senitals. 


\section{CHAPTER XXII.}

\section{TREATMENT OF SYPHILIS.}

As syphilis is a general and constitutional disease, dependent upon a specific poison, it is necessary to introduce a remedy into the circulation which will cause either an elimination of the poison or else neutralize it and render it inert and harmless. The drugs which are the mainstay in the treatment of syphilis are merenry and iodide of potash.

Mercury is supposed to have a direct action upon the syphilitic virus, destroying and neutralizing it.

Mercury may be introduced into the organism by three routes:-

I. Through the skin: (a) by inunction; (b) by fumigation.

II. Under the skin by hypodermic injection.

III. Through the intestinal canal.

It is eliminated by the kidneys, intestinal glands, and by the mucous membrane of the mouth and salivary glands.

After a varying quantity of mercury has been given for some time, the blood becomes saturated with it, and this is annomeed ly the occurrence of mercurial stomatitis, which is ushered in by certain prodromal symptoms:-

(a) A coppery taste in the mouth.

(b) An increased flow of saliva.

(c) Slight pain on striking the teeth together.

(d) Slight swelling and sponginess of the gums next the teeth.

In mild cases of ptyalism the symptoms all subside in a few days if the administration of the drug is stopped; but, if an excessive quantity of mercury has been introduced into the body, or if an musual susceptibility to the action of mercury is present, the toximia is indicated by salivation.

In severe cases of salivation the gums and buceal mueous membrane are greatly swollen and ulcerated. The teeth loosen and fill out, the saliva pours out from the month in quantities, even to the extent of several pints in the day, and the breath has an intensely fœtid odor.

In the administration of mercury in syphilis it is escontial to $(3: \vdots)$ 
give a sulticient quantity of the drug to produce ptyalism, or to "touch the gums," as it is usually called, as this symptom indicates that the patient is getting the requisite quantity of mercury to hold the disease in check. But care should always be taken to stop the mercury hefore sativation is induced.

I bad condition of the mouth and carions broken teeth covered with tartar cause the moutl to react prematurely to the influence of mereury, and for this reason the patient should go to a dentist and hare the teeth put in order, before beginning treatment.

Treatment of Salivation.- The foctor of the breath and the ulccration can be best checked by means of chlorate of potash in solution used as a mouth-wash, and it should also be given internally in doses of 20 grains three times a day. The pain in the gums induced by mastication can be lessened by lrushing the gums with a 4-percent. cocaine solution before eating, although in severe cases the patient should be fed upon liquid food. The excessive secretion of saliva is controlled, to some extent, by hypodermic injections of atropine.

\section{MODES OF ADMINISTERING MERCURY.}

Inunction.- The method of rubbing mercurial ointment into the skin is the most sure and effective mode of treating an ordinary case of syphilis, and is the only plan of treatment to use in the presence of grave lesions threatening life or the integrity of vital organs.

The adrantage of inunction is its prompt action in saturating the body with mercury, and it is possible to give three times the quantity of mercury in this way which could be given by the mouth. At the same time the stomach digestion is not interfered with, and the patient can assimilate the maximum quantity of food, and his mutrition is maintained.

The mercnry, through the friction and pressure of rubbing, is pressed into the open mouths of the sebaceous follicles and sweatAncts in the skin. It is lorought gradually in contact with the blood rirculating in the capillaries of the papillary layer in the skin, and is smposed to be converted into bichloride of mercury through comhination with the sodium chloride in the blood. In this soluble form it is thought to pass into the general blond-circulation. The mercury is strued up in the follicles of the skin for a long time after the inunc- 
tions have been discontinued, and can be found eliminated by the urine many weeks after the last inunction was given.

The blue ointment, or unguentum hydrargyrum, is the luest preparation to use, and it is preferable to have it marle after the formulary of the German pharmacopera, which contains Do grains of metallic mercury in every drachm of lard. In ordinary as 1 drachn is a suitable dose, but in exceptional cases 2 drachms may be necrl.

It is well to have the druggist measure out the daily dose of ointment and inclose it in waxed paper. The ointment should be rery thoroughly rubbed into the skin, selecting a fresh portion of the surface of the body for each day's rubbing, and it is not practicable for the patient to rub himself, but the inunetion should be made by an attendant or masseur.

It requires from twenty to thirty minutes' firm rubbing with the uncovered hand to cause the complete absorption of the entire quantity of ointment used.

It is also desirable to make the inunctions in a regular course, as follows:-

First day: In the calves.

Second day: Inner and outer sides of the thigh.

Third day: Chest and abdımen.

Fourth day: Flexor surfaces of arms.

Fifth day: Back.

On the sixth day the patient takes a warm bath with soap. and on the serenth day begins again with another course of rubbings.

It requires from 20 to 50 inunctions, as a rule, to induce ptyalism, and the gums should be touched in erery case before the inunctions are discontinued. The inunction should be used in both secondary and tertiary periods whenerer danger threatens a vital organ, such as the eye, brain, larynx, etc., and also in obstinate ulcerative processes with rapid destruction of tissue. Inunction is also the most reliable means of treating the ordinary mild cases of syphilis.

Fumigations of calomel are a useful adjunct to other treatment in the cases of early lesions, which are extensive and have a tendency to involve the deeper structures, ulcerate, and extend. The calomelfumes come directly in contact with lesions, and the local action of the vapor of mercury facilitates their healing.

Method of Application. - The patient sits on a cane-bnttomed chair with a blanket around his neck. falling to the floor and surrounding him in a sort of tent. The head is left nnevered. A tin 
fan holding boiling water is placed under the chair, and the patient steamed for fifteen minutes. The pan is then witlydrawn and 30 yrains of calomel are fumigated on a tin stand, orer a spirit-lamp flaced underneath the chair, and the patient is allowed to rcmain surrounded by the fumes for half an hour. The fumigation should be employed once a day until the gums are tonched.

Sublimate baths are employed in the same class of cases, viz.: extensive, ulcerating, suppurating lesions. The intact skin does not fremit a trace of sublimate in solution to be absorbed, but absorption can talie place through solutions of continuity.

Intramuscular Injections.-The former practice of injecting corrosive sublimate in solution under the skin has been practically abantoned; its place is taken by the insoluble salts: calomel and silicylate of mereury, held in emmlsion and injected deep into the sulstance of the gluteal muscles. An ordinary hypodermic syringe is used, provided with a needle which is two inches long and with a thick bore. In ordinary cases the injection is made once a week, and, in coneral, the salicylate of mercury is to be preferred to the calomel. Formula:-

R Iydrargyri salicylatis . . . . . . . . . . . . .

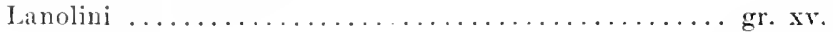

Oleum oliræ........................... m.

II. Sig.: Inject fifteen minims hypolernically once a wetk. Fifteen minims contain gr. iss of the salicylate of mercury.

The alruntages of intramuseular injections are that they act promptly and rapidly, and are almost, if not quite, as efficacious as inunctions. The dosage is accurate, and the injection is only made once a week, so that the patient is reliered from the annoyance of taking medieine or making inunctions in the intervals.

The disadrantages are the pain, which is very inconsiderable, and the slight chance of the occurrence of abscess, which seldom happens.

In exceptional cases the oil has been known to form a pulmonary cminlus, with a localized pneumonia, but recorery followed in each (aitse.

Sulivation may ocenr in a small proportion of cases, without any previons warning, and, as the deposit of mercury is in the muscle, we cannot stop its absorption mless an incision is made into the muscle and the small mass of mereury remored by a curette.

Administration of Mercury by Mouth.-While in Germany the 
usual method of administering nercury is by inunction, in this comntry it is the custom to treat syphilis by its internal anministration.

In recent times, however, we are coming to the conclusion that it is not enough to rely wholly upon giving mercury by the month, but that its action should be supplementul by recular comres of innetion at different times, during the progress of the discase, in order to attain a cure of the syphilis.

If meremy is given by the mouth, after a certain length of tine, the absorptive powers of the intestinal canal often become impiared, and the mercury passes through the alimentary tract mehanged, and without being absorbed. Its continnous use for a long period of time often causes anæmia, emaciation, and diarrhara.

The internal administration of mereury is appropriate during the intervals of inunction and in the periods of latency of syphilis, but it should never be depended upon in severe cases or grave complications. These require the mereury to be given by inmetion or hypodernic injection.

Preparations of Mercury.-Pills of protiodide of mercury (Lamonreux \& Garnier), ${ }^{1}$ /5 grain each.

Method of Administration.-One pill is given three times a day after eating, and every third day the dosage is increased by one pill. For example, the patient takes 3 pills for 3 days, and on the the day he takes 4 pills, and on the Sth day the dose is increased to 5 pills, and so on up to the point of tolerance, which is wsully from 10 to $1 \mathrm{~J}$ pills or more.

The point of tolerance is not manifested by ptralism, except in rare instances, but by diarrleca and cramps. When these simptoms occur, the dosage is reduced to a point just short of producing cramps.

According to the recommendation of Keyes, the full dosage is continued until a period of latency occurs, when it is rertuced to onehalf the number of pills and continued. If a relapse takes plice, the maximum dose is given again.

Another plan of treatment, which is more to be commender, is to ascertain the maximum dose by gradually increasing the mmmber of pills, and, after the maximmm dose is reached, it is maintained. without reduction, throughont the whole conroe of the disease, miess anæmia, emaciation, diarrhrea, or salisation are profuced by it.

Mercury with Chath (Hoydrartyrum cum Crth).-Chis is a mild preparation, and is not apt to indnee colie. and for that reason it may be given when the protiodide produces too much dirmbura. The 
method of administration is similar to that of the protiodide. It may be given in 1-grain pills and increased to the point of toterance, which is manifested by either ptyalism or diarrhea.

lichloride of Mercury.-I)ose, $1 / 30$ to $1 / 15$ grain. This form of mercury is rery useful, and is of particular value, given in combination with iodide of potash, later in the disease in the form of the so-called mixed treatment.

Tannate of Hercury.-Dose, $1 / 2$ to 1 grain three times a day. This is one of the newer preparations, and it is said to have the advantage of causing very little irritation to the intestinal canal.

Iodide of Potash.-The iodide of potash has no direct action in destroying the virus of syphilis, and consequently it is of no use early in the disease. Its action is to cause the absorption of the new growth infiltrating the arteries and which, when it occurs in other tissues, is known as the gumma. The iodide in small doses acts as a tonic, and increases appetite, nutrition, and tissne-change.

The mpleasant effects which are induced by iodide of potash are corys:a and lachrymation, and an eruption upon the skin, which usually occurs upon the back, chest, and face as small acneiform pustules. In rare cases large bulle may form or the eruption may be humorhagic in character and resemble purpura.

In addition, there is often a condition of anæmia, wealness, and general malaise induced. These symptoms are all less apt to occur if the lidneys secrete freely and the iodide is rapidly eliminated.

Dose and Administration.-The only rule for the size of the dose is the effect produced upon the lesion. For the ordinary routine treatment, during a period of latency, 30 or 40 grains a day are enough, but, in the presence of a grave lesion of the nervous system or viscera, $1 / 2$ ounce or even 1 ounce in the day may be required to save life.

Ioride of potash is best given in saturated solution, 1 minim of distilled water representing 1 grain of the salt. It should be given largely diluted with water, and preferably two hours after a meal, as the iodide combines with the starch in the stomach and forms iodide of starch, which is inert.

When the drug disagrees with the stomach and disturbs the digestion, it may be given in milk, which is coagulated with essence of pepsine, or the patient may be directed to drink one or two glasses of hot rater immediately after taking the iodide.

Zittmann's Decoction.- - This is one of the official preparations of the plarmacopaia, and is composed of a number of regetable bitters 
together with a minute quantity of metallic nercury. Its action is first purgative and later tonie, and in some way, which is not mulerstood, it exercises a most beneficial eftect in indolent, sprealing ulariattions which do not respond to mereury and ioclide ol' justasls. 'linese lesions chiefly oecur in malignant syphilis aflecting perouns of foulde vitality, who are usually of the tubercular diathesis and who mumot tolerate mereury or iodide of potash in any consirlerible quantitins.

The hot springs of Arkansas and Aachen in Germany al llseful in the same class of cases, viz.: infeterate syphilis occurring in fuchle individuals whose susceptibility to mereury and iodides is so extrune that they cannot be administered in sufficiunt roses to holl the disuliee under control. The water of these springs contains rery little minclarl substance, but has a temperature of $1 \pm 0^{\circ} \mathrm{F}$. as it issues from the earth. Its action is to cause free secretion by the kidners and skin, and, while it has no specifie action upon the lesions of sphilis, it inereases the tolerance of the body and enables the patient to take large doses of mereury and the iodides.

\section{THERAPEUTICS OF SYPHILIS.}

\section{TREATMENT OF DIFFERENT STAGES.}

Primary Stage.--It is now generally held by the best anthorities that it is not good practice to begin the administration of mereury in syphilis until the eruption appears upon the skin, announcing the commeneement of the secondary stage. If mereury is given hetore this time, it has only the effect of delaying the apjearance of the rash, and it may be postponed for some months, but the premature alministration of mereury has no eflect in aborting the discase or mitisiting its severity. On the other hand, it has been noted by Ehrminn that patients who were treated with mereury for some time previns to the appearance of the eruption were more liable to tertiary affectinns than in the cases where treatment was not begun motil the sucomlany period.

Another advantage which is derived from waiting till socmulary manifestations appear, before beginning treatment, is that the diannosis is fully established and the patient, heine contirely convineed that he has syphilis, is more willing to earry nut laithfully the details of a protracted course of treatment. 
The period of waiting for secondary manifestations can be utilized by sending the patient to a dentist to have the teeth put in order. He should also be instructed as to the hygiene to be maintained during the course of the discase. Everything should be done to maintain the bodity health and nutrition; plenty of sleep and exercise in the open air, abundance of plain mutritious food, and freedom from orerwork or anxiety should be insisted upon.

The use of tobacco should be interdicted, as it causes an irritation to the mucous membrane of the mouth, which predisposes to the formation of mucous patches and chronic ulceration and tends to retard their healing.

Alcoholic drinks in strict moderation may be permitted in the shape of small quantities of beer or light wine, taken preferably with the meals. The habitual use of whisky is injurious.

The patient should be instructed with regard to the danger of communicating syphilis to others, through the medium of a cup, spoon. pipe, towel, or other utensil, or by means of sexual intercourse or kissing.

Mild Form of Syphilis.-As the virus of syphilis is being constantly formed and is present in the blood for months, it is necessary, in order to neutralize its effects, to keep the patient continually under the influence of mercury.

Before begiming treatment we should wait until the eruption is well out upon the body, and, as the virus is most abundant in the early stages of the disease, it is always desirable to push the administration of the mereury at first.

A suflicient number of courses of mercurial inunctions should be given to touch the gums, and the drug should then be stopped for a time until the ptyalism has disappeared. During the following six months mereury should be administered by the mouth, and, as a rule, the protiodide pill answers the purpose better than any of the other preparations, as it does not disagree with the stomach.

In many cases, however, after a time the intestinal canal loses its power of absorption, and the mereury is eliminated with the fæces without having passed through the blood. On this account it is always decirahle to stop the internal administration of mereury, after six nr eight months, in order to allow the intestinal tract to rest, and during the interim several courses of inunctions should be made.

It the ond of the first twelve months, unless some indication aplears for it carlier, the administration of iodide of potash is begun, 


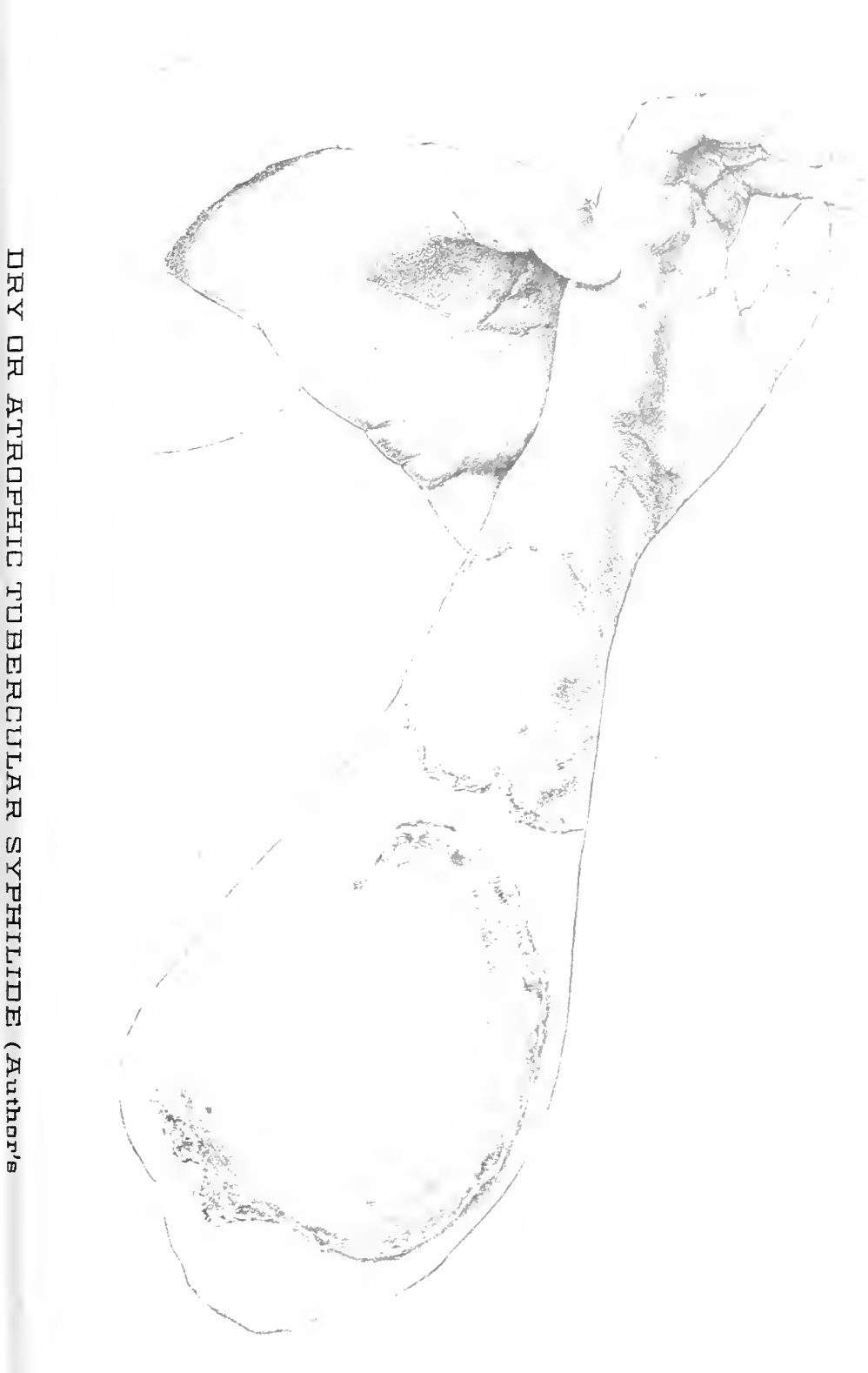



and the mercury is continued in addition. A larorite and u-elul prescription which, although chemically incompatible, is theripututically active, is the following for the so-called mixed treatmont:-

R Hydrargyri bichloridi..................... ij.

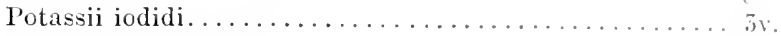

Syrupus sarsaparillat.................. f fo.

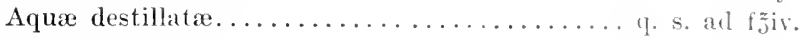

M. Sig.: 3 j t. i. d.

The iodide of potash has no direct action on the virus of syphilis, but it stimulates the activity of the lymphatic system, facilitates. getting rid of waste-products, and also causes the absorption of any syphilitic infiltration which may have taken place in the tissnes or arteries.

The iodide of potash and mercury should be continued tngether with an intermission every six months, during which courses of inunctions should be made. At the end of two and one-half year's treatment the patient having been on mercury alone for twelve months and mercury and iodide of potash together cighteen months lomger, the medication may be stopped.

The patient should be then kept under olsservation six months more, and, if no relapses occur, the syphilis may be considered at an end.

The general nutrition of the patient should receive the closest attention during this protracted treatment. Syphilis itself is a debilitating disease, and mercury, if administered for a long time. has the effect of causing a condition of pallor and andmia. Of course, when such an effect is induced, the mercury should be stopper and tonics given.

Tonics are usually required at some stage in the disense, to counteract the destruction of red corpuscles caused by the virns. The anemia is treated with iron, and the nervons setem and general nutrition are stimulated with strychnia. Codliver-oil is rery raluahle for the emaciation and loss of weight, and esperially so if there is a complication of tuberculosis. Close attention shomlil also lue prail to the matters of fresh air, exercise, foot, sluep, and firmton frum anxiety.

Severe Form of Syphilis.-The serere cates of eylitis and malis-

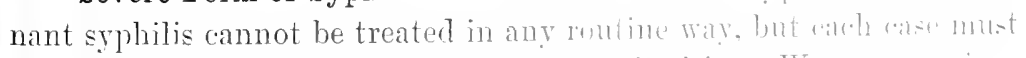
be handled with reference to its om peruliaritics. Tie can saly, in a 
general way, that it is necessary to get the patient promptly under the influence of mercury and at the same time avoid disturbing the digestion, and the lyggienic treatment-consisting in good food, good hygienic surroundings, sunlight, fresh air, and tonics-is imperatively demanded in these cases.

The best method of using mercury is by inunction, and next in ralue may be ranked the hypodermic injections. The disadrantages of giring mereury by the mouth are that it acts more slowly and is very apt to disturb the digestion. After the disease is under control the mercury may be given by the mouth, but inunctions should be used a couple of times a year.

In this class of cases it is necessary to begin the administration of iodide of polash carlier than at the beginning of the second year, inasmuch as tertiary lesions or gummata are apt to occur precociously, even as early as the fifth or six month, and the iodide generally has to be used in larger doses.

After the syphilis has run a severe course for a few months a condition is induced which is known as the cachexia of syphilis, and which is characterized by extreme debility and vital depression, which have resulted in consequence of the anæmia and emaciation.

If severe spreading lesions exist upon the skin and mucous membranes, the case may be properly termed malignant syphilis. These patients unfortunately do not bear mercury and the iodide well, and in these conditions such tonic remedies as Zittmann's decoction and the hot springs of Arkansas are to be recommended.

Grave tertiary lesions of the viscera or nervous system are liable to follow a mild attack as well as a severe one, and, of course, the outlook for ultimate recovery depends largely upon the general character of the patient's constitution.

With individuals of fair bodily health, in the presence of a gumma of the brain or viscera, or of endarteritis of the arteries supplying the nervous system, or, indeed, any of the manifold complications of the tertiary period, the mercury should be given by inunction and iodide of potash in saturated solution. The dosage of the iodide should be increased rapidly up to $1 /$ ounce or even 1 ounce in the dar, the only rule for the quantity given being the effect produced. 


\section{LOCAL TREATMENT OF LESIONS.}

It has been found that the direct contact of a mercurial preparation with a local syphilitic lesion hastens its disappearance by absorption. An advantage is sometimes taken of this fact by using fumigation or bichloride baths, in addition to the regular general treatment, to acclerate the healing of extensive, wide-spread, ulcerating lesions of the skin.

The papular eruptions on the face are annoying and unsightly, and can be made to clcar up more quickly by rubbing in one of the following ointments:-

Oleate of mercury, 5 per cent.

White-precipitate ointment.

Mercurial plaster.

Mucous Patches.-If mucous patches exist in the mouth, they are always of danger to innocent people, since their secretions may be conveyed upon some utensil and be inoculated into another person. On this account we should endearor to heal them as quickly as possible. In addition to the frequent use, by the patient, of a mouth-wash of chlorate of potash or calomel and lime-water, the patches themselves should be touched every three or four days with nitrate of silver in stick, or glycerin and carbolic acid in equal parts, bichloride of mercury in alcohol ( 1 in 20), or the acid nitrate of mereury.

Condylomata heal readily under the general mercurial treatment, and it is only necessary to keep them clean; cover them with a dusting-powder, which absorbs their secretions; and prevent contact with other parts and chafing by means of absorbent cotton interposed. 


\section{CHAPTER XXIII.}

\section{INHERITED SYPHILIS.}

As ALREADY stated in the last chapter, the parental syphilis exerts a bad effect upon the foetus, unless a degree of immunity has been induced. In its most active stages the effect of the poison is to cause the termination of the pregnancy in an abortion; that, as the syphilis grows older its virulence lessens, so that the next child is probably still-born, and the following one may be born alive, but with the syphilitic taint.

Children which are still-born are usually retained long enough in the uterus, after death, to become macerated. The epidermis is stripped off or raised up into large bullæ. The liquor amnii is discolored, brown, and foul-smelling. On examination of the infant's viscera, upon the autopsy-table, they are found to be the seat of the characteristic changes of syphilis.

Occasionally children are born of syphilitic parents who have all the manifestations of a florid syphilis upon them, but usually one or two weeks pass before the syphilis becomes visible. In appearance these children are usually ill dereloped, small, and of light weight. The skin is faded, and they look like little, shriveled, old men.

If the parental syphilis is still older, the children may be born apparently healthy, and develop syphilitic manifestations later. 'The most common time for their appearance is within the first three months after birth and rarely later than the first six months.

Although children born of syphilitic parents may escape the inheritance of an active form of syphilis by reason of a certain acquired inmunity, they may receive a diathetic taint, which does not make it welf evident by any characteristic manifestations. These children have a fecble constitution, and suffer from a general want of mental and hodily development which is particularly notable at the time of puluerty.

The children develop slowly, remain small, and are thin and animic and withont the power of resistance against accidental disease. The intelligence is often deficient, and such instances are described as carces of late hereditary syphilis.

(338) 
COURSE.

The course of inherited syphilis resembles that of the acquired disease, except that it does not begin from a chancre and that the lesions peculiar to the secondary and tertiary periods appear simultaneously.

When a child is born apparently well, but develops syphilis later, one of the most striking symptoms of the impending outbreak is the nasal catarrh, causing snuffles. This is soon followed by an affection of the mucous membrane of the mouth and larynx, which causes a hoarse cry. Mncons patches occur about the skin of the month, which interfere with the child's nursing, and the nutrition suffers.

The eruptions upon the skin resemble those of acyuired syphilis. A macular eruption often occurs upon the chest, and a diffused erythematous redness, resembling eczema in outward appearances, is often seen about the month and navel and parts where the skin is liable to chafe against another opposing surface, such as in the groins, axilla, or nates.

If the patient is not treated, and in severe cases, the eruption develops into papules. The soles of the feet and palms of the hands become the seat of copper-colored papules, which desquamate and are changed into deep fissures. The papules located about the mouth, anus, and genitals are apt to become transformed into luxuriant and vegetating condylomata.

An eruption which is mique, inasmuch as it does not occur in adults, but only in children, is syphititic pemphigus, in which large bulla, or blebs, form on the palms and soles, although it sometimes occurs over the entire body. Its formation is explained by the delicate character of the epidermis and the readiness with which serum collects underneath it and raises it from the derma, forming a resicle, or bulla.

All the above-mentioned eruptions may exist at the same time, and the symptom of polymorphism is usually more marked in inleriter than in acquired syphilis.

The viscera are affected in inherited syphilis eren more frequently than in the acquired form. The liver is often the seat of a form of cirrhosis occasioned by its infiltration with newly-formed connective tissue, or gummata may exist in rarions portions of the gland. The pancreas is affected in a similar way. The lungs may he the site of gummata, and, as a conseqnence of periarteritis, the frame-work of the alveoli becomes infiltrated with new cells in rarinus areas, causing white hepatization. 
A very characteristic feature of inherited syphilis is osteochondritis, which is consinered by some authorities as pathognomonic. It consints in an overgrowth of the cartilage which is interposed between the epiphyes and the diaphyses of the long bones and skull. By lalpation the cnlarged cartilage can ve felt surromnding the bone like a collar. The ultimate course of the inflammation may end in suppuration and necrosis and, after extrusion of the dead bone, the injury is repaired by the abundant formation of new bony tissue, callsing an osteophyte. Osteophytes occurring mpon the skull give a peenliar "squared" slape to it.

The inflammation inrolving the cartilages often travels to the joints, and a serons or purulent synovitis occurs. The teeth of the second set are deformed ly a rertical notching and peg shape of the central incisor's.

The nervous system suffers as well, and epileptiform convulsions, tabes, and progressive paralysis are frequently seen in syphilitic children.

Hæmorrhagic syphilis exists at birth or else makes its appearance within the first month. It is a condition in which the blood is effused under the skin or mucous membranes, forming large purpuric spots. It is not infrequently met with, and is due to the endarteritis, which permits the escape of blood in greater or less quantities.

\section{RECOGNITION OF INHERITED TAINT.}

There are certain points which are of use in making a diagnosis of inherited syphilis at an age advanced from infancy. The most reliable sign is the presence of Hutchinson's teeth. The central upper incisors of the second set are the most characteristically affected. The deformity consists in the peg shape of the teeth and the vertical notching in their lower edges. Jonathan Hutchinson considers them pathognomonic of inherited syphilis, and deseribes them as follows: "The central ineisors are short and narrow, with a broad vertical noteh on their edges and their corners rounded off. Horizontal notehes or furrows are often seen, but, as a rule, have nothing to do with syphilis."

In the subjects of inherited syphilis the skin is thick, pasty, and opaque, or occasionally remarkably soft and silky. At the angles of the mouth may sometimes be noticed linear scars, radiating out into the cheeks. The bridge of the nose is nsually broad and low, and a 


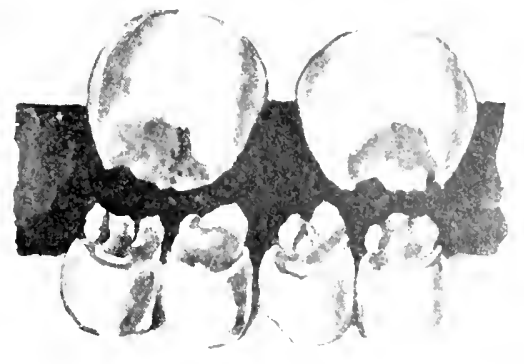

Fig. 94.-Hutchinson's Teeth. These Teeth have heen recently eut, and the Central Notch is well outlined, but the thin and umprotected dentine has not yet crimbled away.

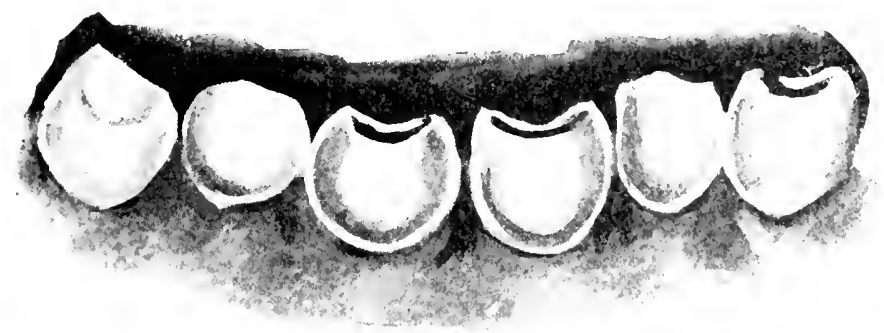

Fig. 95.

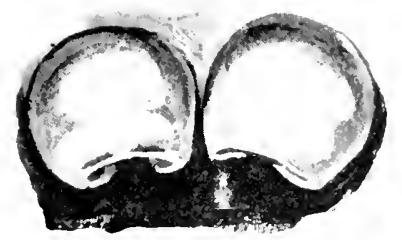

Fin. 96 .

Figs, 95 and 96 Show Later Stages of the Proress after the Dentine has been destroyed. The characteristic Peg shape, with the vertical Central Noteh, is elearly shown. 

want of firmness in the cartilaginous septum, which allows the nose to be shaken about too easily, is sometimes olserred.

The eyes are liable to be affected, and the oceurrence of a wellmarked interstitial keratitis is regarded as palhognomonic of in heriten syphilis. The skull is apt to be squared in shape and to show low protuberances in varions parts. The long bones are often the seat of periosteal thickenings or nodes, and the phalanges and neighloring joints may be affected by a globular swelling, a form of periostitis or ostitis, to which the name dactylitis is given.

The ears are occasionally affected, and synmetrical deafnes. which has occurred withont discharge from the ears, is said to be strong corroborative evidence of an inherited taint.

\section{TREATMENT.}

It has been thought possible to treat an infant, affected with syphilis, by giving mercury to the mother, and allowing the child to nurse from her breast. It was supposed that enough mercury would be eliminated in the milk to control the syphilis of the child. The quantity, however, eliminated in the milk is too small to be of rery much avail, and we are obliged to give specific treatment in other ways.

With children, as in the case of adults, inunctions are the most useful way of giving mercury, as in this way the digestion is not interfered with.

It is not necessary to rub the ointment all over the body, as in adults, but 30 grains of mercurial ointment, mixed with an equal quantity of lanolin to assist absorption, may be spread on the chilits binder, which surrounds the abdomen, and allowed to remain in contact with the skin for two or three days. At the end of that time the ointment may be renewed.

If too much irritation is caused and an eczema follows. the inunctions must be suspended and the drug giren by the mouth. Tarions preparations of mercury may be used in this way.

Among those most highly recommended are the following:--

R Hỵdrargyri cum cretæ...................

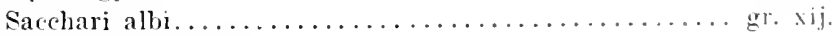

M. et div. in chartulas xij.

Sig.: One t. i. d.

R Calomel gr. $1 / w^{1 / 3}$

Sig.: Three times a day. 
R Bichloride of mercury $\ldots \ldots \ldots \ldots \ldots \ldots \ldots \ldots \ldots \ldots$ gr. $1 / 00^{-1 / 60 *}$

Sig.: Three times a day.

As we have already found, in infantile syphilis the division into secondary and tertiary periods is not clearly defined, and the lesions peculiar to both periods often exist at the same time. On this account iodide of potash is often called for by the appearance of tertiary lesions at an early date in the course of the disease. Iodide of potash may he given in doses of gr. $j$ three times a day, or it may be necessary to give it in much larger doses, if the lesions are severe.

It is eminently desirable to maintain the nutrition of a syphilitic child, and this can best be accomplished by allowing it to nurse from its own mother. The various forms of artificial feeding are less useful, and should only be resorted to when the mother's milk is insufficient.

Duration of Treatment.-No definite rules can be laid down for the length of time required for the treatment. It is considered best to continue for at least two years, with occasional intermissions. but treatment should only be stopped after all manifestations have ceased. 


\section{IMPOTENCE AND STERILITY.}

\section{CHAPTER XXIV.}

\section{IMPOTENCE.}

Impotexce may be defined as an inaljility on the part of the male to copulate, either on account of a failure of the penis to become erect or because the ejaculation of seminal fluid takes place prematurely and before the penis has entered the ragina or else does not necur at all.

The mechanism of copulation is a complicated one, and requires for its performance the co-ordinated working of both nerrous and muscular systems. The function of erection is lnown to be under the control of a collection of nerrous ganglia situated in the lumbar enlargement of the spinal cord, which is called the centre of erection. The centre of erection receives nerve-filaments from the genital centre in the brain, and it also receives sensory fibres from the rectum, bladder, and genitals. It also sends out nerves, the neri erigentes, to the genitals, whose function is to cause a vasomotor paresis of the blood-vessels in the corpora cavernosa.

A knowledge of the distribution of these rarions nerves serves to explain the following faets: An erection may be provoked by the influence of the brain, if it entertains libidinous ideas, or an inhibitory influence may be exerted from the brain by the mental emotions of fear, disgust, or fright (psychical impotence), and vigorous mental activity has the effect of removing, for the time being, sexual desire.

Certain forms of irritation of the spinal cord, eansed by myelitis, traumatism, or fracture-dislocation, when the lesion is located in the cervical or upper dorsal region, are often attended with persistent and powerful ereetions of the penis.

Erections are also caused by peripheral irritation from the genitals, transmitted along the nerves leading to the centre of erection in the cord. At this point, as a result of irritation of the genitals, the sensory impulse is converted into a motor one and reflected back again to the genitals along the nervi erigentes.

As common illustrations of erections from peripheral irritation may be mentioned the erections occasioned by a bladder filled with 
urine in the mornirg, prostatic enlargement, or the passage of a sound through the deep urethra.

\section{MECHANISM OF ERECTION.}

The mechanism of erection is as follows: Under the influence of the nervi erigentes a relaxation of the vascular spaces in the corpora carcrmosa takes place, and they fill witl blood. The penis becomes crect, hard, and elongated, because the blood is pumped into the spaces and retained there.

If the blood flowed ont of the erectile tissue as fast as it came in, erection could not occur, but the swelling of the corpora cavernosa exerts a certain degree of pressure upon the veins which ordinarily conduct the outflowing blood away from the penis. The return-flow of blood is checked by the pressure on the reins, and it is retained in the spaces of the erectile tissue of the penis.

Unless the spaces of the erectile tissue be completely relaxed, a sufficient quantity of blood cannot enter them to exert pressure on the outgoing reins, and the blood flows away through them.

\section{MECHANISM OF EJACULATION.}

In a normal condition ejaculation only occurs with a fully erect penis, except during slcep.

As the spaces of the corpora cavernosa become filled with blood, the verumontanum, or caput gallinagiuis, which is composed of erectile tissue, also swells and becomes erect, thus blocking the entrance to the bladder, so that urine cannot flow out.

The urethral glands secrete freely and a viscid clear drop of mucus appears at the orifice of the urethra. The object of the secretion is to cover the urethral walls bathed in acid urine and to prepare them for the reception of the semen.

The contents of the seminal vesicles are poured out through the ejaculatory ducts until the posterior and bulbous dilatations of the urethra become filled with semen, and after these are distended contractions of the bulbo-cavernosus muscles occur, and the semen is ejaculated in jets from the meatus. If the force of the muscles is impaired, as in paralytic impotence, the semen is not shot out in jets, but dribbles slowly away from the meatus.

\section{CLASSIFICATION OF FORMS OF IMPOTENCE.}

T. ()rganic, from mechanical defects.

I1. Pychical, or imaginary: 1. Complete. 2. Relative. 
III. Atonic, from exhanstion of genital centres in brain and spinal cord.

IV. Symptomatic: Tariety A. Irritable Impotence, from discalse in urethra or adnexa. Tariety B. Paralytic Improtence, from orminic disease in nerrons system. Tariety C. Impotence due to brugs.

\section{ORGANIC IMPOTENCE.}

In this form of impotence some physical cause, which is either congenital or acyured, renders coitus mechanically imposible. The obstacle may prevent the introduction of the penis into the vagina, or, in the absence of a urethra while coitus can be performed. it is not possible to inject the semen into the ragina.

Among the canses of organic impotence may be mentioned such failures of development as hypospadias, epispadias, small size of the organ, or acquired deformities (such as elephantiasis and tumors), disease of the corpora cavernosa (snch as syphilitic or fibroid induration), or partial destruction following wounds, and cavernitis are sometimes responsible for interference with coitus, and operate by causing a deviation or curre in the penis upon erection.

Swellings of the surromding parts, such as hernia, scrotal tumors, or excessive corpulence, with an overhanging belly, may render insertion of the penis impossible, bnt the sexual desire is strong, and ejaculation occurs.

\section{TREATMENT.}

In organic impotence the treatment will be successul in so far as it is possible to remore the mechanical obstacle to enpulation.

In hypospadias and epispadias a plastic operation can be performed, and. when the penis is completely inclosed by orerlying issues, a small, freely-movable penis capalble of intromission may be formed.

Tumors and elephantiasic growths must be removed by surcical means, and hernia and hydrocele also call for operation. An effort should be made to bring about absorption of the infiltration in the corpus spongiosum. which may be confidently expected to occur if it is syphilitic in origin. 


\section{PSYCHICAL IMPOTENCE.}

We have already noted in studying the physiology of coitus, the fact that the brain is capable of exerting a restraining influence over the power of erection, through the inhibitory nerves which go to the spinal centre of erection. As a result of nerrous excitement, the action of the inhilbitory nerves from the brain is aggrarated, and erection fails at the critical moment. The influence of fear and dread are observed in the same class of patients before passing a sound.

When these individuals are lying on the table, the penis is seen to shrink and grow smaller and move in a worm-like manner, which is caused by the spasmodic contraction of the muscular fibres imbedied in the trabecule of the corpora cavernosa. In such cases the inhibitory nerves are stimnlated by the dread of catheterism, and in the same way other psychical influences stimulate the action of the inhibitory nerres, and the patient is at such moments impotent.

Tarious types of men are affected by the form of psychical, or imaginary, impotence, as follows:-

Class A.-A few strong, young, vigorous men who have lead clean lives, on being married to the women of their choice, either from a state of nervous excitement or a lack of confidence in themselves or perhaps occasionally from timidity and bashfulness, do not succeed in holding an erection long enough to perform coitus, and the crection either fails to be complete or else ejaculation occurs prematurely.

Class B.-Feeble, despondent, oversensitive individuals of weak nervous fibre, who have masturbated, had an attack of gonorrhcea, or have been excessive in sexual intercourse. Such persons are continually dwelling on past abuse and worrying orer trifling symptoms, such as a varicocele or the normal weekly occurrence of a seminal cmission, and their fears are aggravated by reading quack books.

Class C.-Men of good health and well-balaneed minds may be affected by various mental emotions, such as fear, disgust, and loathing, or the departure from regular habit, all of which may induce temporary impotence.

It is no uncommon experience, when illicit intercourse is being attempted, that a fear of exposure or infection or of disgust at some coarse remark on the part of the woman canses a failure or sudden subsidence of the erection. 
The effect of habit is seen in the cases where a man is accustomed to cohabit successfully with one woman. Hut fails to accomplish the act of coitus with a stranger, until he becones accustomed to the new fields. Roubaud's case is cited as an example of this. I young minn who was accustomed from the beginning of his sexul life to a certain type of woman, who was a blonde and always met him drescel in a silk gown and with shoes on, conld never have coitus with any other woman, unless she were also a blonde and dreeced in a sinilar manner.

Severe and prolonged mental strain, such as occurs with students and business and professional men, sonetimes lrings about a contition of general neurasthenia, one of the symptoms of which is temporary impotence.

Relative impotence is a form of psychical impotence. and is the term applied to a condition in which the man is able to connlate with certain women only, and not with others.

When this is the case between man and wife, it may he due to a mutual aversion or a lack of scxual feeling on the part of the wife. which reacts upon the man and takes away his desire, and yet the same man may be entirely potent with other women.

\section{TREATMENT.}

Before beginning treatment, it is essential to make sure that we are dealing with a case of true psychical impotence and that the impotence is not symptomatic of some lesion in the urethra or nervous system.

The psychical form disappears spontaneonsly when the mental disturbances occasioning it ranish. For instance, the death of a belored wife or loss of fortune and business anxieties may canse temporary impotence, which disappears as time goes by or the individual's circumstances improve. In these cases the friendly adrice of a physician in whom the patient has confidence is raluable.

The largest number of cases of psychical impotence occur in nerrous young men who have had gonorrhea or masturbated frecly. and who have read and pondered over the false and lurid accounts in quack medical advertisements, pretending to decribe the evil consequences of masturbation or sexual excesses. 


\section{ATONIC IMPOTENCE.}

The term atonic impotence has been rather loosely used by author's, and has been incorrectly made to include cases which were fependent upon lesions of the urethra or spinal cord or the abuse of rrugs, and should have been properly classed under the head of symptomatic impotence.

Atonic impotence is a variety of comparatively rare occurrence, and in which there is no demonstrable lesion of the nerrous system or urethra. It is purely functional in character, and is dependent eutirely upon a failure of the spinal centre of erection, and perhaps the cenital centre in the brain, to respond to ordinary stimuli and canse the penis to become erect.

It should be borne in mind, howerer, that a condition of exhaustion of the nerre-centres is often complicated by chronic inflammatory changes in the posterior urethra, induced by a gonorrhcea of long standing or sexual abuses, and this fact should not be lost sight of in carrying out the treatment. IIere, again, advice and the assurances of the physician, in combination with attention to building up the general health by salt-water bathing, out-of-door exercise, boating, golf, and moderate walking or light gymnastic exercises is the basis of treatment.

II ypnotism may be successful in relieving this form of impotence, but. as our knowledge of influencing the mental powers in this way becomes more extended, the rlanger of inducing the hypnotic state and other drawbacks becomes more obrions; so that, in general, it is not desirable to employ this form of treatment.

In atonic impotenee sexual desire is often present, but frequently it is alsent, and, as a mle. crections do not oecur at all. Sometimes, howerer, in mild cases, a partial erection, accompanied by premature ejaculation of semen, takes place.

The atonic form of impotence is exclusively found in married men who have practiced coitus cxcessively or "withdrawal" with their wives for rears, excessive masturbators, and old roués whose only thonght in life has been the gratification of their sexual desires.

The lack of erectile power is usually only one symptom of general nemiathenia, which is accompanied by its usual signs, viz.: mental symptoms such as imparment of memory, fulhess in the head, and anvisty: pains in the lack and limbs, fechle heartaction, vasomotor disturbinces, indigestion, constipation, etc. 
These general symptoms have been Acscribed ly quarks in terrifying terms, in pamphlets pretending to set forth the "eril elfects of self-abuse," which have an extensive eirculation among the laity.

Authorities are now generally agreed that, aside from a degradition of the moral character of the individual, masturhation, practiced oceasionally, exercises but little bad effect upon the gencral health, and its effects cease as soon as the habit is stopjed, unless a condition of ehronic inflammation is indnced in the posterior urethra, and the same is true of coitus reservatus, or withdrawal.

If, however, masturbation or withdrawal is practiced at frequent intervals and for a long period of time, chronic posterior urethritis occurs, which gives rise to various local symptoms and is often eomplicated by a general neurasthenic state (see "Chronic Posterior Lrethritis").

\section{DIAGNOSIS AND TREATMENT.}

Before making a diagnosis of atonic impotence care shonld be taken to exclude any disease of the urethra and its adnexa or the nervous system. It is generally the case that, with old rounders and masturbators, a stricture, chronic urethritis, prostatitis, seminal resiculitis, or beginning spinal disease is present, and the impotence is not atonic and caused by exhaustion of the nervous centres, but is secondary to and a symptom of the local structural change in urethra or spinal cord.

When all these local conditions have been exchded, however, and we are certain that we are dealing with a pure functional neurosis, the following lines of treatment may be employed:-

The indications are to build up the general health of the patient, and at the same time give complete rest to the genital centres in the brain and cord; so that their cells may have an opportunity to store up again a renewed quantity of nervous energy.

Later on, after a sutficiently long period of rest has been enjoyed, a plan of treatment should be adopted calculated to stimulate and arouse again to activity the dormant cells in the genital centres: but this should never be attempted until a sufficient period of complete repose has been afforded them.

The neurasthenia, which is usually present, requires the first attention, and the patient should be directer to make use of a light, easily-assimilated diet, get plenty of sleepl, and take gentle, regular exercise in the open air. 
Riding on horseback and the bicyele is not to be reenmended, un account of the jarring to which the perineum is subjected when these are indulged in. Sea-bathing lias an excellent effect as a general tonic.

E.pecial stres should be laid upon the necessity for avoiding all sources of erotic excitement, such as lewd books, conversation, and thentrical displays; companionship of women, immoral or otherwise, and all attmpts at scxual interconrse must be rigorously interdicted.

In the early stages of treatment, in addition to blood-and tissuebuilding tonics,--such as codliver-oil and iron,-the spinal sedatives -bromide of potash and lupulin-are in order for the purpose of giving the necessary rest to the exhausted centres.

After this treatment has been continued for some weeks and the patient's neurasthenia has disappeared, it sometimes happens that his sexual functions are improved as well; but frequently the irritability of the genital centre is still so exhansted that erections either do not occur at all or are not sulficiently rigorous, and ejaculation is premature: so that a special stimulating plan of treatment has to be adopted to arouse the activity of the cells in the genital centres.

The drugs which are supposed to be particularly useful as stimulants to the nerrous centres are phosphorus and nux romica. They may be combined in the following manner, as suggested by Gross:-

R Quinice sulphatis,

Ferri sulphatis.................. of each gr. xl.

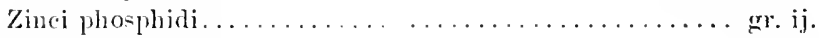

Acidi arsenosi. ..........................

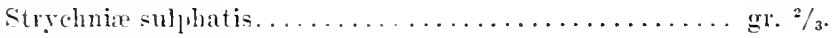

I. et ft. pil. No. xl.

Sig.: Two pills every eight hours.

Damiana and cantharides have gained some reputation among the laity as aphrodisiacs, but damiana has little or no effect, and cantharides acts as an irritant upon the kidneys and hladder whenever taken in doses sufficiently large to act as a genital excitant.

It was hoped that, when the animal extracts were first introduced, they might prove of value, but extended trials of them have only resulted in disappointment.

Electricity enjors a high reputation in the treatment of atonic impotence. The constant current is adapted to most cases, beginning with the positire pole applied orer the lumbar region and the negative 
pole used to stroke the penis, testicles, and perineum. The strength of the current may be gauged by the sensitivenes of the patient, and it should not be used strong enough to cance discomfort. The sittinge at first should be for two or three minutes every forty-eight hours, and soon increased to five minutes daily. In obstinate cases, if erections do not occur from these applications, the positive electrole may be introduced into the rectum and a more powertul effect excited. In cases where marked anæsthesia of the skin of the genitals exists. the faradic current may be used, with better results than the gralranic.

Cold douches or the alternate use of cold- and hot-water doucher, applied with some force to the spine daily, are of some astitince. and in the same way irrigation of the rectum with hot water throuch a Kemp tube may be of some service as a local stimulant to the prostatic urethra.

Local treatment of the urethra is, of course. demanded whon atonic impotence is complicated by chronic inflammation of the posterior urethra (see "Symptomatic Impotence"): but, in cases where the posterior wrethra is healthy and the difficulty is a pure neurosis, the passage of sounds and use of instillations generally increase the nenrasthenia, and the patient is made worse.

The operation of ligation of the dorsal vein of the penis ha- been practiced, with the object of retarding the return-flow of hlood, and so allowing the erectile tissue of the corpora cavernosa to become listended and filled up with blood, in the cases where a partial erection occurred, but where the penis almost immediately became flaccid without any discharge of semen. A fer cases have been reported where this operation proved successful, but the mental effect of an operation may have been partly responsible for the good result.

After the condition of impotence has yielded to protracted and careful treatment, and the patient is again able to have crections and ejaculate normally, he should be warned against indnlging too frecly in sexual intercourse, for any excess in this line will be very apt to be followed by a recurrence of his former disability. 


\section{SYMPTOMATIC IMPOTENCE.}

This is by far the most frequent type of impotence met with in practice. The disability is never primary, as in the other forms, but it is always dependent upon and secondary to certain structural patholncrieal changes, located in the nervous system or the urethra and its arnexa, and the inability to copulate is only one symptom of many which go to make up the clinical picture.

ln symptomatic impotence erections may be completely absent, although, as the genital centres in the nerrous system are not affected, sexual desire is usually present.

In many cases a partial erection of the penis takes place, and the seminal fluid is ejaculated prematurely. This form is generally spoken of as

\section{VARIETY A. IRRITABLE IMPOTENCE.}

This term is derived from the fact that, on account of the irritable state of the posterior urethra, the ejaculation of semen oceurs liematmrely before the penis has penetrated into the vagina, and the organ, which nerer attained to a complete erection, becomes flaccid immediately after the discharge. Many of these cases, which are characterized at first by premature ejaculation, grow worse, and in time the power of erection disappears entirely.

Irritable impotence generally depends upon the presence of chronic posterior urethritis, and the inflammation often extends from the urethra through the ejaculatory ducts, and the prostate and scminal resicles become affected also.

Chronic posterior urethritis originates from a gonorrhœa or from sexual excesses, masturbation, or the pernicions habit of interrupted coitus, or "withdrawal."

These causes are liable to induce a condition of chronic engorgement of the blood-ressels in the posterior nrethra, and, in course of time, hyperplasia of the submucous connective tissue occurs, and the newly-formed scar-tissue, pressing upon the delicate nervous structures of the verumontanum, occasions various reflex symptoms in the hrain, nervous, and muscular systems. The process is often further complicated by germ-infection, often the bacillus coli, which aggrarates the existing conditions.

Instead of a chronic posterior urethritis heing responsible for the reflex disturbances, an organized stricture may be present in the pend- 
ulous wethra, and, if the stricture he located in the dewere portion of the canal, it interferes so much with the circulation of blood, in the posterior urethra, that a chronic conwetion results, which disapperis promptly when the stricture is treaterl.

\section{TREATMENT.}

To treat the symptom of irritable impotence succosfrully, the condition in the posterior urethra requires attention. (ree " thunic Posterior Urethritis," "Prostatitis," and "Sominal T toiculitis.") The discharge of pus, if present, should be controlled br irrigutions, and later, when shreds alone are found floating in clear urine. instillations of nitrate of silver in the posterior urethra are called for.

The submucous infiltration can be favorably influenced lor the passage of large-sized sounds. An instrunent of particular wificaley in reducing the irritability of the posterior urethra is the psyeturophor, which combines the benefits of pressure with the ellects of colli in blunting the hypersensitiveness of the nervous system.

If the prostate or resicles are diseated, masage and expresion ot their contents, by means of the finger in the rectum, are required to bring about a resolution of those affected organs, and, if a stricture is present, it must receive appropriate treatment, either by dilatation or cutting.

The prognosis of irritable impotence is good in so far as we can succeed in removing the cause upon which the impotence depends.

\section{VARIETY B. PARALYTIC IMPOTENCE.}

In paralytic impotence erections do not talie place at any time, although half-erections sometimes occur. Ejaculation does not necur at all, or else takes place withont cansing any sensation, and the semen is mot ejected in jets, but gradually oozes from the meatus.

On examination the genitals are found withered and flaccirl. and the skin of the penis is but slightly sensitive, often quite andethutic. The sensitiveness of the urethra is diminished, and a sound mathe passed with ease and without pain.

Paralytic impotence. as its name implies, is dopentent upun organic cerebral or spinal disense. which causes paralysis in othor pints of the body. Blows upon the lack of the head are especially liable to be followed by complete impotence.

In loenmotor ataxia in the carly staces, there nuy be a conclition 
of priapism; but, as the case progresses, the sexual powers decline, until finally the patient may be completely impotent. Myelitis in mild forms does not affect the sexual powers, but in severe forms a state of impotence is induced.

The prognosis in paralytic impotence is, of course, bad, and treatment, either local or general, is of little or no use.

\section{VARIETY C. IMPOTENCE RESUITING FROM DRUGS.}

Sexual power is sometimes diminished or entirely destroyed from the excessive use of ccrtain drugs. Individuals who are addicted to the habitual and excessive use of opium, chloral, bromide of potash, and hashish are very apt, in time, to lose sexual inclination and power to copulate. Workmen who are exposed to the exhalations of certain chemicals-such as arsenic, antimony, and lead-are affected in the same way.

The influence of an excessive quantity of alcohol in reducing sexual power is a matter of common linowledge, but we are not so critain as to the effects of tobacco, although some authorities claim that tobacco has an effect in lessening both sexual inclination and capacity.

An excess of sugar in the blood, which occurs in diabetes, also causes impotence, although the individual's health and strength are apparently not deteriorated.

The treatment of impotence resulting from the use of drugs consists, of course, in removing the cause, and, when this can be done, the prognosis is, in general, favorable.

\section{STERILITY.}

Until recent years a condition of sterility was always thought to be due to some pathological condition on the part of the woman which prevented conception. It is now recognized that in a small number of cases, although the man is capable of performing the sexual act, still his semen is destitute of the fecundating element, and he is sterile.

As an example of this state may be cited the case of men who have been castrated. After this operation the subjects do not become impotent at once, but, for a year or two after the testicles have been 
removed, are able to practice copulation and ejaculate a matcrial composed of urethral mucus and secretions from Cowper's and the prostate glands, but devoid of spermatozon, and hence incapable of impregnating the female.

The composition of normal semen, after ejaculation, is found to consist of spermatozoa formed in the testicles and the scretions of the seminal vesieles, prostate, Cowper's glands, and the glands of the urethral nucous membrane, viz.: Morgagni's crypts and Littés glands.

The usual quantity of semen ejaculated at one discharge is from 5ii-iv; but, if coitus is repeated frequently, the yuantity becomes smaller each time, until finally only a few drops are prodnced with difficulty.

The essential life-giving element in semen is, of conrse, the spermatozoa, and the function of the other ingredients is probally to coat the urethral mucous membrane and dilute the semen.

On microscopic examination the spermatozoa present a most striking picture, showing numerous, small, tadpole-shaper bodies moring actively in the field. They continue to show movements until at least twelve hours after evacuation, and will present signs of activity for forty-eight hours if sheltered from light and cold.

The semen also contains spermatic cells, which are supposed to be breeding-places for the spermatozoa, one of which, according to Kölliker, develops out of each nucleus of a cell. Böttcher's crystals are discovered on adding a few drops of 1-per-cent. solution of phosphate of ammonia, and appear as variegated groups of dagger-shaped crystals. It is supposed that the organic base of the cristals exists in the secretion of the prostatic follicles, and gives the semen its characteristic odor.

Finger classifies the pathological changes in the semen which cause sterility as follows:-

I. Azoöspermia, or absence of spermatozoa.

II. Oligozoöspermia, or a marked diminution in the number of spermatozoa.

III. Necrospermia: The spermatozoa are dead and without motion.

IV. Aspermia, entire absence of semen: 1. Absolute. 2. 'Temporary. 


\section{AZ0 ÖSPERMIA.}

In this condition there is an entire absence of spermatozoa. 'The physical character of the semen is not distinguishable from normal; the oftur, consistence, and color are unchanged; and the absence of spermatozoa can only be detected by the microscope.

Azoüspermia is, of course, the normal condition before puberty, but it is rarely found in old men, who usually are able to form a few spermatozoa.

The pathological canses which bring about this condition may be grouped as follows:-

(a) Frequent seminal emissions, either from excessive masturbation or too frequent coitus, cause, first, a diminution in number, and, if persisted in, a complete disappearance of spermatozoa, which reappear in the semen again after a few days' rest.

(b) Disturbance of the Secreting Function of the Testicle.-This cenerally occurs in the acute ferers, and the debility following convaleccence, and, as a result, the spermatozoa disappear temporarily from the semen. In chronic diseases the same often occurs, and azoinspermia often exists in tuberculosis and in syphilis, even when the testicles are not affected.

(c) Disease of the testicle, caused by syphilis, tuberculosis, or malinnant discase, if it is bilateral and destroys completely the parenchyma of the organ, interferes with the formation of spermatozoa and leads to sterility. It is necessary, however, that it should be a complete destruction, for, if a small part of the secreting portion is left, spermatozoa may still be formed.

(d) Absence of both testicles-either from removal by castration. atrophy consecutive to orchitis, congenital absence, or atrophy following ectopia testis-will, of course, induce permanent and irremodialjle sterility.

(e) Bilateral obstruction of the vas deferens is the commonest caule of azoöspermia, and results from epididymitis, which in 90 per cent. of the cases is due to gonorrhcea. Tuberculosis and syphilis are responsilile for the remaining 10 per cent. The obliteration of the vil: deferens is caused by the pressure, upon the seminiferous tubes, of a mass of inflammatory scar-tissue in the tail of the epididymis, which contracts, and squeezes the tubes together. Finger cites 242 cases of domble epididymitis, out of which $20 \%$ suffered from azoöspemina. 
The treatmeut of azoöspermia seldom meets with much success, except in cases of syphilitic epirdidymitis. Here an energetic course of antisyphilitic medication will often cause alsorption of the infiltration, and the testicle resumes its function.

In gonorrhœal epididymitis the infiltration is hard and dense, and after it has existed some little time it is impossible to bring alout its absorption. It is important, for this reason, to treat every case of gonorrheal epididymitis carefully, in order to avoid the danger of sterility (see "Epididymitis").

\section{OLIG0Z0ÖSPERMIA.}

This condition consists in a marked diminution of the number of the spermatozoa, and may, in general, be regarded as a temporiry condition, which, depending upon its cause, either returns to the normal state or goes over into complete absence of spermatozoa (azoöspermial).

Oligozoöspermia occurs normally in old age and at the beginning of puberty, and it also occurs in general debility from any discase and also after repeated acts of sexul intercourse. 'The most usual canses for it are gonorrhœal epididymitis or new growths-either syphilis. tuberculosis, or cancer-which involve the epididymis.

As long as the vasa deferentia are not completely closerl by the inflammation, a few spermatozoa may make their way through the canal. If the spermatozoa are diminished in number, and at the same time motionless, sterility is assured; but, if the spermatozoal retain their movement, there is always procreative power left, Lut in a lessened degree.

\section{NECROSPERIIA.}

In this condition the male is able to copulate and to ejaculate semen, but on microscopic examination the spermatozoa are fouml to be dead and without motion. In order to make a ralirl test. it is necessary to examine the semen not later than one to two hours after ejaculation, and the specimens can only be secured by directing the man to have coitus while wearing a condom. 
Neerospermia is brought about by a variety of causes which diminish the secreting capacity of the testicle. Exccsses in venery or unduly frequent seminal emissions operate in this way. At first the semen is normal, but in time it becomes thinner, the numbers and motility of the spermatozoa diminish, and they become small, deformed, and unripe.

The same eflect is produced upon the spermatozoa by a disturbance in the nutrition of the testicle from alcoholism, morphinism, aneral tulerculosis, or diabetes. Tarious local processes-such as syluilis, carcinoma, and beginning atrophy-exert their effect upon the parenehyma of the gland, and the formation of spermatozoa is atfected in consequence.

I more frequent cause of the death of the spermatozoa is some pathological alteration of the component parts of the semen. Inflammation of the seminal vcsicles, either acute or chronic, causes perceptible alteration in the semen. It is usually purulent and sometimes bloody, deriving its foreign constituents from the inflamed carities of the resicles, and the spermatozoa are found to be dead.

The spermatozoa are also motionless in cases of chronic follicular prostatitis, for, as Furbringer has demonstrated, the spermatozoa, so long as they are retained in the seminal vesicles, are motionless, and it requires the contact of the prostatic secretion to arouse their normal motility. When the prostatic follicles are diseased, their secretion is cheeked, and the spermatozoa are deprived of the stimulant necessary to excite their activity.

As already indicated, the treatment of neerospermia will depend upon the cause which occasions it. and the prognosis is good or otherwise as we are alıle to remove its origin.

\section{ASPERMIA.}

Aspermia may be defined as a condition in which the male is able to perform coitus properly, but no semen is ejaculated into the vagina of the female, either because none is secreted or becalise some obstruction in the urethra prevents its passing from the meatus.

Aspermia may be either absolute or temporary, congenital or acquired.

Congenital aspermia is a very rare condition, hut a few cases have 
been reported. Ultzmann assumed it to be due to a non-excitability of the reflex centre of ejaculation. Jacobson suggests that, while the testicles are present and capable of forming spermatozoa, they calnnot malie their way into the urethra on account of an occhusion of the ejaculatory ducts or an absence, from anomaly of derelopment, of a portion of the ras deferens.

Acquired aspermia is not uncommon and is often the result of suppurative affections of the prostate brought alout by gonorihua or tuberculosis. Through the destruction of the gland and the smlsequent formation of scar-tissue, the ejaculatory ducts are closed by the contraction of the cicatrix. If one duct is left open, the semen is diminished in quantity, but not noticeably so; but, if both ducts are closed, aspermia follows.

The ejaculatory ducts are also occasionally destroyed, during a lateral lithotomy, either by being cut in incising the posterior urethra or by suffering laceration in dragging a large stone out of the bladder and through the wound.

The ejaculatory ducts sometimes become plugged by the formation of concretions composed of spermatozoa, mucus, epithelial cells, and lime-salts.

An insensitive condition of the glans penis may be responsible for the failure of ejaculation, and cases have been reported where an injury to the spine caused a complete anæsthesia of the skin of the genitals. In another case on record the prepuce and dorsum of the penis had been destroyed by ulceration and converted into a large indurated scar, which was entirely insensitive.

One of the most frequent causes of aspermia is stricture of the urethra. On account of the swollen and turgid condition of the mucons membrane of the urethra during coitus, the orifice of a tight stricture is closed, and the seminal fluid is unable to escape past it, but is retained in the urethra behind the stricture. After the congestion subsides the verumontanum no longer eloses the resical outlet, and the semen regurgitates into the bladder, and is subsequently discharged with the urine.

Temporary aspermia is a rare condition which occurs in persons of a nervous temperament who hecome nemasthenic from excesses in venery, masturbation, or gonorrhoa. Such individuals are nsually impotent (psychical impotence), but occasionally such patients are found who can copulate, but eannot ejaculate any semen at the time, although the seminal fluid may escape a few hours later during sleep, 
as an emission. This form of aspermia begins suddenly, lasts a few weeks or months, and then vanishes as suddenly as it came.

The treatment of aspermia in its various forms depends upon the etiology, but the variety depending upon stricture offers a good prognosis when the urethra is restored to its normal calibre. 


\section{LIST OF GENITO-URINARY INSTRUMENTS REQUIRED FOR OFFICE USE.}

VALENTINe's irrigator.

Ultzmann's syringe.

Large hard-rubber or glass syringe. Capacity, + to 6 ounces.

Oberlaender or Kollmann's dilator, antero-posteror.

Otis urethrometer.

Eighteen steel sounds, Tan Buren curve. Numbers 16 to 34 French, inclusive, but omitting every other number.

Benique sounds, 16 to 34 inclusive, onitting every other number.

Tunneled sounds, numbers 12, 14, 16, 18, and 20 Frencll.

Psychrophor with Benique curve; attached to four-quart douchebag.

Bougies a boule, metal; numbers 16 to 32 inclusive, omitting every other number.

Guyon's flexible gum-elastic bongies a boule, 16 to 30 French, omitting every other number.

One dozen whale-bone guides.

Thompson's searcher for stone.

Nitze's observation cystoscope.

Six Klotz endoscopes (Eissner \& Co., New York): 3 four inches long. Calibre, respectively, 26, 2S, and 30 French. One five and onehalf inches long. Number 26 French. Two six inches long. Numbers 28 and 30 French.

Head-mirror.

Oil-lamp (Belgian or other circular wick, with central draft).

Long urethral forceps.

Iypodermic syringe for injecting mereurial salts (Eissner \& Co., New York).

Catheters: Soft rubber. Silver, with prostatic curve. Mereier condé and bicoudé (prostatic). One Gouley tunneled silver catheter, English, with stylet.

Flexible bougies from number 18 down to smallest. 



\section{INDEX.}

Alopecia, 29s.

Anatomy of the urethra, 16.

Aspermia, 360.

acquired, 361 .

congenital, 360.

temporary, 361.

Azoöspermia, 358.

causes of, 358 .

Racteriuria, 154.

course of, 155.

diagnosis and treatment of, 155 .

prognosis of, 155.

symptoms of, 155 .

Balano-posthitis, 5.

diagnosis of, 6 .

symptoms and course of, 5 .

treatment of, 6 .

Bladder, avenues through which micro-organisms reach the, 137.

benign tumors of the, 156 .

malignant tumors of the, 156 .

solutions for washing the, 149 .

tumors of the, diagnosis of, 157 .

prognosis of, 158.

symptoms of, 157.

treatment of, 158 .

Bubo, 280.

course of, 281.

differential diagnosis of, 281 .

etiology of, 280.

operative treatment of, 282.

treatment of, 281.

Calculi, oxalic, 159.

phospluatic, 159 .

uratic, 159.

Calculus in children, operations for, 180.

renal, 229 .

colie as a srmptom of, 230 .

diagnosis of, $23^{2}$.
Calculus, renal, distulnances of urinary function ats a symptom of, :31. etiology of, 2:-29.

gastro-intestinal distubames accompanying, 231 .

hamaturia as a symptom of, $232 \mathrm{I}$. pain as a srmptom of, 231 .

passage of framents in, :231.

prognosis of, 232 .

pyuria as a symptom of, 231 .

symptoms of, 230 .

treatment of, 233 .

vesical, 159 .

blood as a symptom of, 166 .

caurse's of phosphatic, 165 .

diagnosis of, $161 \%$.

etiology of, 160.

increased freqnency of urination as a sympitom of, 16 its.

litholapaxy in the treatment of, $170^{\circ}$

number of, 159

operative treatment of, 170.

pain as a symptom of, 165.5

perineal lithotomy in the treatment of, 173.

lithotrity in the treatment of, 174.

predisposing causes of urativ and oxalic, 160 .

preventive treatment of, Its.

sudden stoppage of the urine as a symptom of, 16 ti.

srmptoms of, 165 .

the cystoscope in the diagmosis of, 16 s.

the litholanaxy pump in the diagnosis of, lis.

(ancer of the penis, 12 .

amputation of the entire penis for, 14.

free purtion of the punis for, 11. 
Cancer, conlse of, 13. diagnosis of 13. etiology of. 12. operations for, 14. pognosis of, 13. treatment of, 14 .

C'aput gallinaginis, 21.

Catheter, Mercier, 200.

the silver prostatic, 200 .

Cluancre, $2 s+$. course of, 254 . definition of, 284. diagnosis of the, 285 . differential diagnosis of, 289. duration of the, $28 S$. heals without learing a scar, $2 S S$. length of time after, at which infection of the fœtus is liable to occur, 322 .

of the uretlura, 288 .

pathology of, 256 .

prognosis of, 290 .

transmission of contagion of, 285 .

treatment of. 290 .

vaccination, $28 \overline{5}$.

varieties of, $28 \%$.

C'hancroid, 271.

characteristics of, 272 .

complicated by paraphimosis, 279.

by phag diena, 279 .

complications of, 278 .

course of, 272 .

diaguosis of, 276 .

duration of, 275 .

etiology of, $2 \pi 1$.

frequeney of, 272 .

microscopic examination of, 275.

moles of contagion of. 272 .

subpreputial, complicated by phimosis, $27 s$.

treatment of, $2-6$.

Chordee, 26.

Circumeision, 3.

by dorsal ineision and trimming off the tlaps. 3.

with a clamp, 3.

Compressor urethra, 16.

Condyloma. :28t7

Cytitis, 134.
Cystitis, acute, batsams in the treatment of, 145 .

diluents in the treatment of, 144 . general treatment of, 144 .

hot sitz-baths in the treatment of, 144.

instillations of nitrate of silver in, 146.

local treatment of, 146.

opium in the treatment of, 145.

purgatives in the treatment of, 144.

the diet in, 144 .

urinary antiseptics in the treatment of, 145 .

chronic, general treatment of, 146 .

local treatment of, 147 .

perineal drainage in, $14 \pi$.

solutions for washing the bladder in, 149.

suprapubic drairage in, 148 .

classification of, 138.

constitutional symptoms in, 141 .

diagnosis of, 142 .

etiology of, 134.

frequent urination in, 140 .

gonorrheal, 150.

hæmaturia in, 141.

membranous, 139.

micro-organisms found in, 135 .

painful urination in, 140 .

pathological changes in, 135.

preventive treatment of, 143 .

prognosis of, 143 .

pyuria in, 141.

simple, 138 .

specific, 150.

symptoms of, 140.

tuberculous, 150 .

diagnosis of, 151 .

involvement of the bladder in, 152.

microscopic examination of the pus in the diagnosis of, 152.

modes of infection in. 150.

pathological changes in, 151 .

prognosis of, 152 .

simptoms and course of, 151 .

the cystoscope in the diagnosis of, 151. 
Cystitis, tuberculons, treatment of, $15 \%$. Cystoscope, the, in the diagnosis of tuberculous eystitis, 151.

in the diagnosis of tumors of the bladder, 157.

Cystotomy in the diagnosis of tumors of the bladder, 157 .

Dilator, Oberlaender, 59.

Ejaculation, mechanism of, 346 .

Epididymis, channels through which tubercle bacilli are conveyed to, 247.

Erection, mechanism of, 346 .

Fossa navicularis, 21.

Genito-urinary instruments required for office use, 363 .

Gonococcus, Gram's method of detecting, 29 .

morphology of the, 28 .

Van den Bergh's method of detecting, 28 .

Gonorrhea, 23.

acute, balanitis as a complication of, 75 .

chordee as a complication of, 76 . complications of, 75 .

Cowperitis as a complication of, 76.

epididrmitis as a complication of, 79 .

folliculitis as a complication of, 75.

inguinal adenitis as a complication of, 76 .

paraphimosis as a complication of. 75 .

phimosis as a complication of, 75 .

anterior, adrantages of Janct's method in, 37 .

astringent injections in. 33 .

disadrantages of Janet's method in, 37.

irrigations with permanganate of potash in, 35 .

Janet's method in, 35 .
Gonomhea, anterior, methol of using the salts of silver in, 39.

terhnique of Janet's method in, 37.

the abortive treatment of, 35.

the sillts of silver in, 28 .

treatment of the stage of decline of, 32.

causes which retard recuvery from, 30.

course of, 26 .

duration of an attack of, 29 .

endoscopic examination of, 26.

examination of urine in, 27.

healing of the lesions of, 25.

microscopic examination of pus in, 27.

pathology of, 23.

prodromal stage of, 26 .

relapses of, 25.

treatment of, 30 .

Gonorrhœal rheumatism, 81 .

treatment of, $8 \%$.

Gumma, 310.

of the skin and subeutancous tissiles, 310 .

Hæmatocele, 264.

etiology of, 264.

of the spermatic cord, 265 .

symptoms and course of, 264 .

treatment of, 264 .

Herpes progenitalis, 8.

diagnosis of, 8 .

etiology of, 8.

treatment of, 8 .

Hydrocele, 255.

acute, 255.

etiology of, 255.

pathology of, 2.55 .

purulent, 256 .

symptoms of, 256.

treatment of, 256.

chronic, 256 .

incision of the sac for, 262.

pathology of, 259 .

puncture of, 261 .

radical treatment of, by injection, 261 .

symptoms and diagnosis of, 260 . 
Iy ylrocele, chronic, treatment of, 260 . cumprenital, 25\%.

of the spermatie cord, 263.

treatuent of, ?(i3.

Hydroncphrosis, 239

diagnosis of. 241 .

ctiology of, 240.

intermit teut, 240 .

prognosis of. $2+1$.

symptoms of $2+1$.

treatment of. 242.

Impotence, 345 .

atonie, 350 .

diagnosis and treatment of, 35 .

clasitication of forms of, 346 .

irritable, 354 .

treatment, 355 .

organie, 347 .

treatment of, 347 .

paralytic, 355.

psychical, 348 .

treatment of, 349 .

relative, 349 .

resulting from drugs, 356 .

symptomatic, $35 t$.

Kidney, moralle, :226.

diagnosis of, 228 .

etiology of, 226 .

prognosis of, $2 \cdot 28$.

symptoms of, 227 .

treatment of, 228 .

surgical, 234 .

true floating, 226 .

Kollmam's posterior dilator, 65.

Lithola paxy, mortality of. 173.

Lithotomy, perineal, median oferation of, 174 .

mortality of, 176 .

suprapulice, 176.

mortality of, 179 .

Littre. glands of, 21.

Mixed sore, 275.

Morgagni, follicles of, 21.

Nucous patch, 39\%.

Muscle, ent-olf, of the urethra, 16.

Neerospermia, 850

Nephrectomy. 233.

Neplirolithotomy, 233.
Olserlaender dilator, 59.

Oligozoöspermia, $3 \overline{5} 9$.

Osteophyte, 310.

Papillomata, 11.

diagnosis of, 11.

treatment of, 11.

Penis, cancer of the, 12.

Phagedæna, serpiginous, 280.

sloughing, $2 \mathrm{~s} 0$.

Phimosis, l.

acquired, 1.

congenital, 1.

direet results of, 1 .

remote results of, 2 .

temporary, 1.

treatment of, 3 .

Profeta's law of immunity, 321 .

Prostate, diminution in size of, after castration, 210.

hypertrophied, Alexander's operation of perineal prostatectomy for, 206.

Bottini's operation for, 203 .

castration for, 210 .

choice of operations in, 217 .

clinical results of castration for, 211.

Fuller's operation of suprapubic prostatectomy for, $20 \mathrm{~s}$.

mortality of castration for. 213.

operative treatment of, 202 .

palliative operations for. 216 .

perineal opening for, 216.

prostatectomy for, 205.

prostatotomy for, 205.

puncture with a trocar for, 217.

remote results of castration for, 214.

results of suprapubic and perineal prostatectomy for, 20!.

suprapubic cystotomy for, 216.

suprapubic prostatectomy for, 207.

vasectomy for, 215.

senile hypertrophy of the, 181.

atrophy and distension of the bladder in. 192.

changes in kidneys and ureters in, 193. 
Prostate, senile, changes in the ureter in, 189.

changes in the wall of the bladder in, 191.

commencement of catheter-life in, 199.

continuous catheterization in, 200.

cystoscopic examination in the diaguosis of, 194.

diagnosis of, 194.

form of obstruction in, 182.

gemeral treatment of, 195 .

hypertrophy with contraction of the bladder in, 19:.

incontinence of urine in, 192 .

pathology of, 181.

rectal examination in the diagnosis of, 194.

residual urine in, 191.

retention of urine in, 193, 199.

saccular dilatation of the blad. der in, 192.

suprapubic aspiration of the bladder in, 200.

symptonis of, 190.

the quantity of residual urine in the diagnosis of, 194.

tuberculosis of the, 221 .

diagnosis of, 223 .

pathology of, 222 .

prognosis of, 224 .

symptoms and course of, 222.

treatment of, 224.

Prostatitis, acute, 84 .

follicular, 84 .

chrome, 87 .

diagnosis of, 88 .

pathological anatomy of, 87 .

prognosis of, 90 .

symptoms of, 87 .

treatment of, Ss.

parenchymatous, course and termination of, 85 .

diagnosis of, 86 .

symptoms of, 85 .

treatment of, $S 6$.

simple acute, 84 .

Prostatorrhœa, Si.

Psychrophor in the treatment of chronic prostatitis, 89 .
Psychrophor, the, 65.

l'yelitis, 234 .

diagnosis of, 236.

etiology of, $23 \pi$.

prognosis of, $2: 37$.

symptoms of, 23.5.

treatuent of, $23 \mathrm{~s}$.

Pyelonephritis, 234.

Rhenmatism, gonorrhoal, sl.

Salivation, treatment of, 325 .

Sinus pocularis, 21 .

Sound, the Benique, 65.

in the treatment of chronic prostatit is, s!.

the cold water, 65 .

Sterility, 356.

Stone, sounding for, 166.

Stricture, annular, $10+$.

cicatricial, 104.

complicated with a false passage, 126.

inodular, 104.

intractable, treatment of, by resection of a portion of the urethra, 127 .

linear, 104.

of the meatus, 126 .

of the urethra, 102 .

organic, 102 .

abscess of the kidney in, 107 .

changes in bladder and kidney in, 106.

changes which take place belind the, 105.

continuous dilatation in treatment of, 116.

dangers of internal urethrotomy in, 120 .

definition of, 102 .

diagnosis of. 108 .

distension of kidneys in, 107 .

of ureters in, $10 \%$.

distorted stream in, 107 .

dribbling after urination in. 107.

effects upon, of passing sounds, 114.

etiology of, 103.

external urethrotomy in, 121. 
Stricture, organic, false passage in, 110. flexible bulbous bougie in diag. nosis of, 108 .

frequent mination in, 107. gleety dischange in, 107.

Gouley's tumneled catheter in, 117.

gradual dilatation in, 110.

hyclronephrosis in, 107.

hypertrophy of bladder-wall in, 106.

immediate operation in, 117 .

interference with coitus in, 108 .

internal urethrotomy in, 118.

location of, 104.

netal bulb in diagnosis of, 109 .

numbers of, 104.

Oberlaender dilator in treatment of, 115 .

of very small calibre, 116.

Otis's methrometer in, 109.

pain in the urethra in, 108.

pathology of, 103.

pouching of urethra in, 105.

pyelitis in, 107.

relapses of, 116 .

resilual urine in, 107.

retention of urine in, 107 .

summary of indications for internal wrethrotomy in, 120.

symptoms of, 107.

treatment of, 110.

by surgieal operation, 117.

tumeled sound and gradual dilatation in the treatment of, 116.

uleeration in, 105.

varieties of, 103.

vesical atouy in, 107.

whalebone filiform guides in the diagnosis of, 109.

spasmodic, 102.

canses of. 102.

tortuous, 10 t.

Syphilicle, macular, 299.

papular, 209.

papulo-pustular. 299.

papulo-squamous, 299.

liguentary. 299.

pustular, 299. syphilide, rupial, 313.

tubereular, dry or atrophic, 313.

ulcerative form of, 313 .

Syphilides, 29s.

absence of burning and itching in, 30 s.

color of, 308 .

distribution of, over the body, 300 .

Syphilis, 292.

abortion of, after infection, 291

administration of mereury by the mouth in, 330.

and irritation, 314.

and marriage, 323 .

and the eruptive fevers, analogy between, 292.

cachexia of, 336 .

carriers of the poison of, 292 .

course of virus of, through the lymphatic system, 296 .

diaguosis of, 324 .

duration of secondary stage of, 308 .

fumigation by ealemel in, 329 .

glandular enlargement of, 295.

hereditary, late, 338 .

immunity in, 319.

incubation of, 295 .

infection of fœtus in, 320 .

influence of the child's, as exerted upon the mother, 321.

inherited, 320, 338 .

affections of the nervous system in, 340 .

affections of the viscera in, 339 .

condylomata in, 339 .

course of, 339.

duration of treatment of, 344 .

eruptions upon the skin in, 339 .

hemorrhagic, 340 .

Hutchinson's teeth in, 340 .

interstitial keratitis in, 343 .

osteochondritis in, 340 .

smuffles in, 339 .

treatment of. $3+3$.

inoculation and eourse of, 293.

intramuscular injections in, 330 .

inumetion of mereury in, $32 \mathrm{~s}$

iodicle of potash in, 332 .

loeal treatment of lesions of. 337.

malignant, 314 .

mercury in the treatment of, 327. 
Syphilis, mode of increase of the rirus of, 294 .

modes of administering mercury in, 328.

preparations of mercury used in, 331.

prodromal symptoms, or prodromata, 296.

Profeta's law of immunity in, 321. prognosis of, 325 .

recognition of inherited taint of, 340.

secondary, 296.

albuminuria as a prodromal symptom of, 297 .

alopecia of, 298.

classification and anatomy of, 299.

condyloma of, $29 \vec{\imath}$.

course of the eruption of, 307 .

erythema of the fauces as a prodromal symptom of, 297 .

fever as a prodromal symptom of, 296 .

jaundice as a prodromal symptom of, 297 .

mucous pateh of, 297 .

nocturnal pains as a prodromal symptom of, 296 .

skin eruptions of, 298.

stages of, 294 .

sublimate baths in, 330 .

tertiary, anatomical changes in, 309.

characters of lesions of skin and mucous membrane in, 309.

characters of skin eruptions of, 310.

gummatous arteritis in, 309 .

pathology of, 309 .

stage of, 308 .

the hot springs of Arkansas and Aachen in, 333.

the virus of, 292 .

therapeutics of, 333 .

treatment of, 327 .

mild forms of, 334 .

primary stage of. 333 .

severe forms of, 335 .

Zittmann's decoction in, 332.

Syphilitic eruptions, relapsing, 300 .
Syphilitic erythema, 299.

nother, termination of pregnancies in a, 322.

roseola, 299 .

Testicle, ectopy of the, 243 .

complications of, 244.

diagnosis of, 245 .

etiologr of, 244 .

results of, 244 .

treatment of, 245 .

galloping consumption of the, $2+9$.

malignant disease of the, 246 .

clinical history of, 246.

prognosis and treatment of, $24 \pi$.

varieties and description of, 246 .

syphilis of the, 252 .

diagnosis of, 253 .

prognosis of, 253 .

symptoms and course of, 253 .

treatment of, 254 .

tuberculosis of the, 247 .

castration for, 251 .

course of, 248 .

erosion or curetting for, 2.j1.

operative treatment of, 251 .

prognosis of, 250.

symptoms and diagnosis of, 250 .

treatment of, 251 .

Urethra, anatomy of the, 16 .

anterior, 16.

normal appearance of, 69 .

aseptic eatarrh of the, 22 .

bulbous, 21.

cut-off muscle of the, 16 .

membranous, 16.

mucous membrane of the. 21.

normal appearance of. 70 .

physiological narrowing of the, 21.

points of widening of the. 21 .

posterior, 21.

prostatic. 21 .

Urethral instruments, are of. 132.

Urethritis, acute, 22.

predisposing caukes of, $2 \underline{2}$.

anterior, 30.

ehronic, ti.

changes in the mucosa in, 49.

dialgnosis of, $5 \%$. 
Lrethritis, anterior, chronic, final lieal-

ing of the hesoms in, 50 .

ghandular changes in, 48 .

instillations in, $5 \overline{5}$.

isolated foci of 55 .

method of nsing Otis's ure-

thromcter in, jot.

passage of steel sound in, 58 .

pathology of, 47 .

symptoms ct, 51 .

treatuent of, 54 .

treatuent of the deep form of, $5 \mathrm{~s}$.

treatment with the endoscope in. 58.

Ultzmann's apparatus in, 57 .

formule for astringent injections in, 33.

methodic treatment of, 30 .

technique of injecting in, 33 .

the use of injections in, 34 .

therapentic treatment of, 31 .

treatment of the increasing stage of, 31 .

chronic, 4 ti.

anterior and posterior, prognosis of 67 .

anterior and posterior, summary of treatment of, 66 .

method of examining a case of, 68.

pathological changes in, 70 .

predisposin $\vec{u}$ causes of, 46 .

gonorrheal, 22.

posterior, 41.

acute, 41.

clironic, 41, 61 .

diagnosis of, 62 .

instillations in. 64 .

irrigation in, 64.

passage of sounds in, 65.

pathology of, 61 .

symptoms of, 63 .

treatment of, 64 .

diagnosis of. 42 .

subacute, 41 .

symptoms of, 42.

treatment of the mild form of, 43.

treatment of the severe form of,
Urethritis, simple, 22.

treatment of, 23.

specitic, 22.

Urethroscopy, 68.

Ijrethrotomy, combined external and internal, 127.

externai, louley's operation with a guide, $1: 1$.

Guitéras's trocar in, 124.

recontraction of the stricture after, 126.

retrograde catheterization in, 124.

treatment after, 12.5.

Wheelhouse's operation without a guide, 123 .

Urinary fever, 130.

acute, 130.

chronic, 130.

etiology of, 130.

prognosis of, 130 .

treatment of, $1: 31$.

Urine, extravasation of, 128.

treatment of, 129 .

Uterus masculinus, 21 .

Varicocele, 265.

diagnosis of, 260 .

etiology of, 265.

excision of a portion of the reins for, 269 .

subcutaneous ligation of the reins for, 269.

symptoms of, 266 .

treatment of, 269 .

Verumontanum, 21 .

Vesiculitis, atonic, 92, 95.

chronic, with perivesiculitis, 96 .

withont perivesiculitis, 96 .

inflammatory, 92 .

seminal, acute, 91 .

diagnosis of, 91 .

symptoms of, 91 .

treatment of, 92 .

chronic, 92.

etiology of, 96 .

diagnosis of, 98 .

symptoms of, 97 .

tuberculons, 99.

treatment of, 100. 



UNIVERSITY OF CALIFORNIA LIBRARY

Los Angeles

This book is DUE on the last date stamped below.

Form L9-10m-5,'67(H2161s8) 4939 

wh of

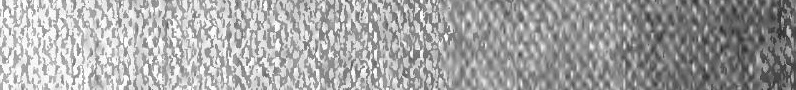

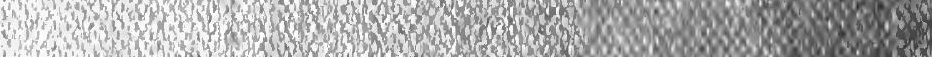
and

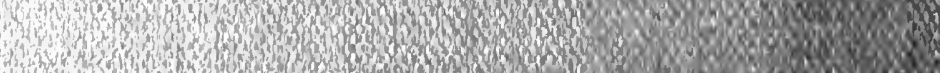

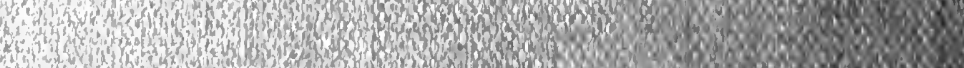
1)

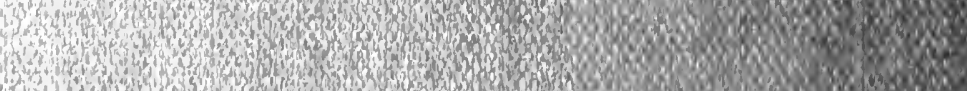

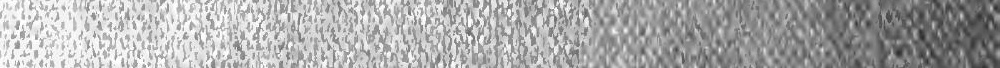
A. to 1 in

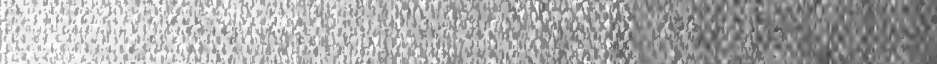
Why

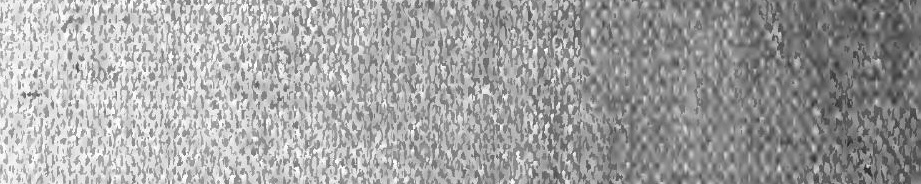

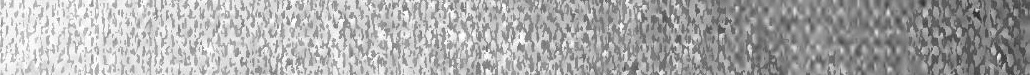
3. 4. 5 of

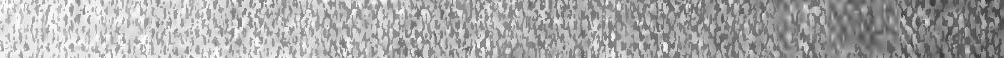
W. 20.ther 1. Q. Ch w

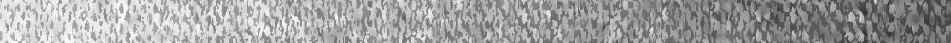

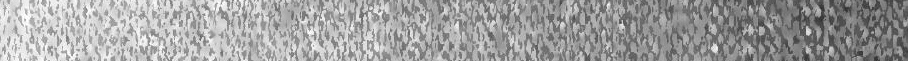

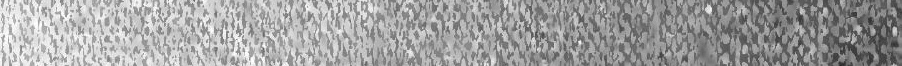
(5)

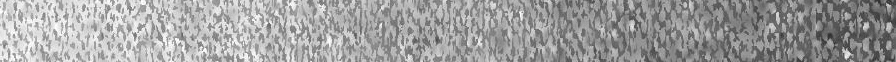
3.t. H.

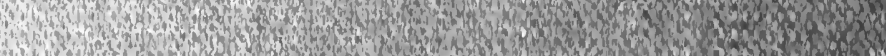
Q 5, 1 .

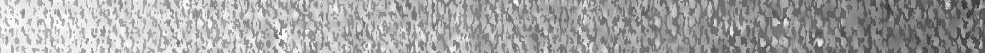

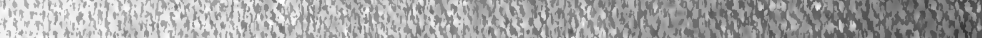
1.

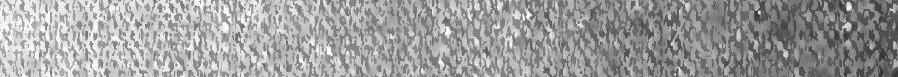
4 int in 1.t. 19. H. W. 19. Atis in

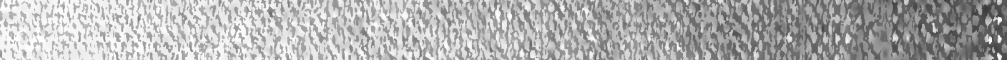
Whis

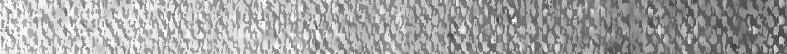
fint How

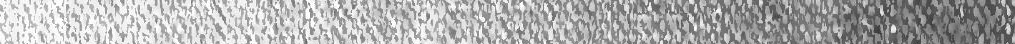

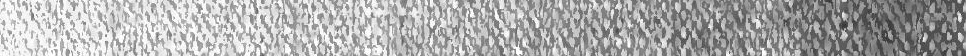
Wh W 1.

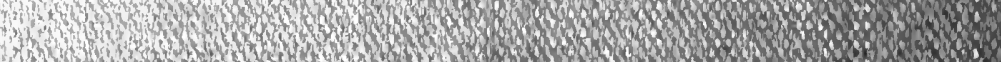

